\title{
In vivo morphometric and mechanical characterization of trabecular bone from high resolution magnetic resonance imaging
}

\section{Ángel Alberich Bayarri}

\author{
Valencia (Spain), 2010
}


The research described in this thesis was carried out between October 2007 and June 2010 at the Radiology Department of the Hospital Quirón Valencia (Valencia, Spain). The project was financially supported by Vicerectorat d'Innovació $i$ Desenvolupament of the Universitat Politècnica de València (PAID-06-07/3104); Grupos emergentes Generalitat Valenciana grant (GV/2009/126); Instituto de la Mediana y Pequeña Industria Valenciana (IMPIVA) of the Generalitat Valenciana (IMIDTP/2009/334); and Spanish Ministry of Science through project No. TEC2009-14128. 


\section{In vivo morphometric and mechanical \\ characterization of trabecular bone from high resolution magnetic resonance imaging}

Ángel Alberich Bayarri

Dissertation submitted

in partial fulfillment of the requirements for the degree of

Doctor in Philosophy

Supervisors:

Dr. David Moratal Pérez

Center for Biomaterials and Tissue Engineering Universitat Politècnica de València, Valencia, Spain

Dr. Luis Martí Bonmatí

Radiology Department, Hospital Quirón Valencia \& Hospital Universitario y Politécnico La Fe, Valencia, Spain

Dr. María Ángeles Pérez Ansón

Aragon Institute of Engineering Research (I3A),

University of Zaragoza, Zaragoza, Spain

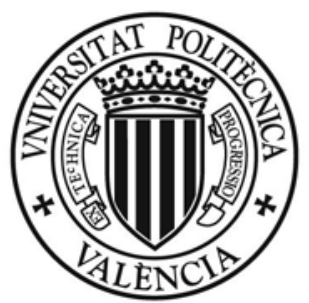

Universitat Politècnica de València 

A mí me interesa todo lo que supone trabajo y voluntad; a mí me interesa toda persona que tiene un ideal y procura realizarlo. “El Papa del mar”, Valencia 1925. Vicente Blasco Ibáñez (1867 - 1928) 


\section{Acknowledgements}

It is my pleasure to gratefully thank all the people that made this thesis possible; they are all key participants of this work.

First of all, I would like to express my gratitude to my three Ph.D. advisors, Dr. David Moratal, Dr. Luis Martí-Bonmatí and Dr. Mª Ángeles Pérez. I would like to initially thank Dr. David Moratal for pleasantly receiving me with open arms when I was looking for collaboration in biomedical engineering applications. He offered me to be in charge of the investigation in image processing applied to osteoporosis and trabecular bone. David, thank you for opening the doors of research and medical imaging for me, you have been not only a great advisor, but also a faithful companion.

My research was physically being performed at the Radiology Department of Quirón Hospital. I will never forget the first time I crossed the 'no entry' glass doors of the department to meet Luis and start planning my work; at that moment I felt that it was going to mean a before and an after in my life. There, I also discovered that everything can be planned in a small napkin. As a lover of medicine, mathematics and whole science, Dr. Luis Martí-Bonmatí is one of those advanced minds that support the combination of medicine and engineering, a reference not only in research but also in human behavior. Thank you Luis, for being my mentor and giving me the opportunity of enrolling in this exciting professional career.

I needed support in computational mechanics and discovered my 'hero from the silence' in Zaragoza, Dr. M ${ }^{\mathrm{a}}$ Ángeles Pérez. It is difficult to overstate my gratitude to $\mathrm{M}^{\mathrm{a}}$ Ángeles for being so involved in the thesis supervision and for all the support I have received from her. Thank you $\mathrm{M}^{\mathrm{a}}$ Ángeles for your continuous motivation to my work and for your friendship.

During my last three years working at Quirón Hospital, I have shared many experiences, like traveling on a big ship, and have many things to thank the rest 
of the crew for. First of all I would like to make a special mention to my colleagues of the quantification group (CQV): Roberto (now Dr. Sanz), Gracián, Carlos and Amparo, thank you all for the continuous support and motivation in my work and for the idyllic atmosphere we have created together, long life to CQV! I also want to express my gratitude to Merche, our projects assistant, thank you for all the encouragement you have given to me. One of the best experiences has been working side by side with the team of radiologists; I am indebted to Salva, Juani, Cristina, Vicent, Rosario, Elena, Paula, Toni and Enrique for their numerous lessons of medicine and for their continuous messages of support. Also, I must acknowledge the work of the team of nurses and technicians in MR images acquisition. The support of the administrative staff, with a special mention to Dani, must be also recognized. I would like to include Dr. Juan José Lerma of the department of Rheumatology in these acknowledgements, for his difficult task in recruiting osteoporosis patients. Finally, I would like to express my gratitude to Sergio and Sonia, as hospital managers and also to Antonio, as corporate director, for their support in my work.

It is also a pleasure for me to express my sincere gratitude to the people from Philips Healthcare, who have provided great support to the thesis. Thank you Javier for your permanent collaboration and for those unforgettable moments in front of the magnet improving MR sequences. I have learned a lot from you. Han, Carmen and Fran, thank you for encouraging me in my work.

I would like to cite here a group of friends who are very special for me: Víctor, Pedro, José Antonio and Pere, the best industrial engineers of Zaragoza. It all started in a summer course in El Escorial, Madrid. The charm of the place defined a friendship that has grown stronger and stronger through time. We are now mutual hosts either in Zaragoza or Valencia; friends, thank you so much for your support and for introducing me to the finite element method. I would also like to thank you for introducing me to $\mathrm{M}^{\mathrm{a}}$ Ángeles and Dr. Manuel Doblaré, who deserves my admiration and gratitude for helping me with the 
definition of the main thesis topics and for letting me to use the micro-CT of his group.

I would like to show my appreciation to Dr. Julio Carballido from the University of California San Francisco, for his collaboration in my work about fractals, and also for giving me the opportunity of investigating together in new skeletonization methods. I am also indebted to Dr. Thierry Metens, from the Université Libre de Bruxelles, Belgium, for his contributions and remarkable suggestions to my work.

The gratifying support of Montse and José Vicente from the IBIME group at Universidad Politécnica de Valencia must be also recognized. We have a long way to run together.

On a personal level, I would like to thank all my friends from Benicarló and Valencia for giving me high doses of support and very good moments of relaxation. A special mention must be done to Fede for stopping the thief that was stealing my bag containing all my Ph.D work.

I wish now to thank my family and specially my parents, Paula and Ángel, they not only gave me life, but also fill it with all the affection and love one could wish for. They have always encouraged and supported me to do my best in all matters of life. Thank you.

Finally, Blanca, that girl I met at high school ten years ago and blocked my heart spins like a strong magnetic field. There are not enough words to say thank you. You have been always encouraging me by my side, always with a smile, with an optimistic point of view, even in the hardest times of my work. You are me and I am you. There are not enough words, no words...

My in-law family deserves my sincere gratitude: Sonia for her continuous interest in our progresses; Pepita, for offering me a place to cut the sheep bones and also Mariano, my favorite star in the sky. 



\section{Contents}

$\begin{array}{lr}\text { Abstract } & 1\end{array}$

Resumen 3

Resum $\quad 5$

$\begin{array}{ll}\text { Motivation } & 7\end{array}$

$\begin{array}{ll}\text { Objectives and structure } & 10\end{array}$

$\begin{array}{lr}\text { Chapter 1. Introduction } & \mathbf{1 2}\end{array}$

1.1 Clinical scenario: Bone and Osteoporosis 12

1.1.1 The bone tissue 12

1.1.2 Bone development and remodeling 15

1.1.3 Osteoporosis 17

$\begin{array}{ll}\text { 1.2 Magnetic resonance imaging } & 21\end{array}$

1.2.1 Physical principles 22

1.2.2 System architecture $\quad 25$

1.2.3 Pulse sequences 29

1.2.4 MR in the study of bone $\quad 32$

1.2.5 Acquisition requirements for trabecular bone

1.3 Image processing and quantification of trabecular bone 35

1.3.1 Image processing in digital radiology. Applications to the study of trabecular bone 35

1.3.2 State of the art in trabecular bone characterization.

Quantification of trabecular bone from MR images 36

Chapter 2. Methodology 42

2.1 Image acquisition $\quad 42$

2.1.1 Region of acquisition $\quad 42$

2.1.2 MR Hardware $\quad 42$

2.1.3 MR pulse sequence $\quad 44$

2.2 Image processing 46

2.2.1 Segmentation $\quad 47$

2.2.2 Coil heterogeneities correction 53

2.2.3 Sub-voxel processing 54

2.2.4 Binarization $\quad 55$

$2.33 \mathrm{D}$ reconstructions of the trabeculae 56

2.4 Morphometry analysis $\quad 59$

2.4.1 Morphology 59

2.4.2 Complexity 63 
2.4.3 Topology 67

$\begin{array}{ll}2.4 .4 \text { Anisotropy } & 75\end{array}$

2.5 Mechanical analysis using the FE method 82

2.5.1 Generation of a voxel-based FE model 82

2.5.2 Mechanical simulation of compressive conditions $\quad 88$

2.5.3 Processing of the results 91

2.6 Reproducibility and validation of the measurements 92

2.7 Study groups 95

2.8 Statistical analysis 96

$\begin{array}{lr}\text { Chapter 3. Results and discussion } & \mathbf{9 8}\end{array}$

3.1 Evaluation in a healthy population. Normality values 98

3.1.1 Morphometry results in healthy subjects 98

3.1.2 Mechanical results in healthy subjects 107

3.2 Assessment of morphometry and mechanical alterations under pathological conditions in osteoporosis disease 114

3.2.1 Morphometry results in osteoporotic patients $\quad 115$

3.2.2 Mechanical results in osteoporotic patients 127

3.2.3 Morphometry and mechanical relationships

with bone mineral density $\quad 130$

3.3 Reproducibility analysis 132

$\begin{array}{ll}3.4 \text { Validation of the measurements } & 134\end{array}$

$\begin{array}{lr}\text { Chapter 4. Conclusions } & 139\end{array}$

$\begin{array}{lr}\text { Chapter 5. Future work } & 142\end{array}$

5.1 Evaluation of patients with different ethiopathogenesis $\quad 142$

5.2 Longitudinal analysis of the morphometry and mechanical parameters in a group of patients under treatment 143

5.3 Use of MDCT for the trabecular bone characterization 143

5.4 Mechanical simulations of trabecular bone in the non-linear regime 144

5.5 Study of cortical bone water using ultra-short echo times $\quad 145$

5.6 Imaging based evaluation of bone deposition on polymer scaffolds in tissue engineering $\quad 147$

5.7 Development of an integrated image post-processing platform 147

$\begin{array}{lr}\text { References } & 149\end{array}$

$\begin{array}{ll}\text { Publications } & 161\end{array}$

$\begin{array}{ll}\text { Abbreviated terms } & 165\end{array}$

$\begin{array}{ll}\text { Appendix } & 168\end{array}$ 



\section{Abstract}

Osteoporosis is a bone disorder that manifests a decreased bone density and also deterioration in the cancellous bone architecture. Both factors increase bone fragility and the risk to suffering bone fractures, especially in women, due to their higher prevalence. The current diagnosis of osteoporosis consists in the quantification of the bone mineral density (BMD) by means of dual energy $X-$ ray absorptiometry (DXA) techniques. However, BMD alone does not entirely account for fracture risk or therapeutic effects. Other factors such as the microstructural disposition of the trabeculae and their characteristics need also to be considered in order to determine bone quality and directly assess the fracture risk.

The technical advances of medical imaging modalities such as multi detector computed tomography (MDCT), high-resolution peripheral quantitative computed tomography (HR-pQCT) and magnetic resonance imaging (MRI) have allowed for the in vivo acquisitions at high spatial resolutions. The trabecular bone structure can be observed with good detail using these techniques. In particular, the use of 3 Tesla (T) MR scanners has permitted the acquisitions at higher spatial resolutions. Furthermore, the good bone to marrow contrast obtained in MR images as well as the use of non-ionizing radiations set MRI as a suitable technique for the in vivo trabecular bone characterization in osteoporosis disease.

In the present thesis, new methodological developments for a threedimensional (3D) morphometry and mechanical characterization of the trabecular bone are proposed and applied to high resolution 3T MR acquisitions. The morphometry analysis is compound by different algorithms designed to quantify morphology, complexity, topology and anisotropy parameters of the trabecular tissue. Regarding the mechanical characterization, new methods for the automated simulation of the trabecular bone structure 
under compressive conditions and the calculation of the elastic modulus were developed.

The developed methodology was applied to a population of healthy subjects in order to obtain normality values of the cancellous bone. The algorithms were also applied to a population of patients with osteoporosis in order to quantify the variations of the parameters due to the disease and evaluate the differences with the results obtained in an age-matched healthy group.

Finally, an evaluation of the reproducibility of the methods was performed by the calculation of the morphometry and mechanical parameters from repeated MR acquisitions performed to 5 sheep tibiae in three consecutive days. The validation of the measurements was also assessed by a comparison of the results with the same sheep tibiae acquired at higher spatial resolutions by micro computed tomography $(\mu \mathrm{CT})$.

The proposed methodological developments and clinical applications provide satisfactory results, with a high sensitivity of the parameters to trabeculae variations mainly influenced by sex and the osteoporosis status. Furthermore, the methods present a high reproducibility and accuracy in the morphometry and mechanical parameters quantification. These results may reinforce the use of the presented parameters as imaging biomarkers of osteoporosis. 


\section{Resumen}

La osteoporosis es una enfermedad ósea que se manifiesta con una menor densidad ósea y el deterioro de la arquitectura del hueso esponjoso. Ambos factores aumentan la fragilidad ósea y el riesgo de sufrir fracturas óseas, especialmente en mujeres, donde existe una alta prevalencia. El diagnóstico actual de la osteoporosis se basa en la cuantificación de la densidad mineral ósea (DMO) mediante la técnica de absorciometría dual de rayos X (DXA). Sin embargo, la DMO no puede considerarse de manera aislada para la evaluación del riesgo de fractura o los efectos terapéuticos. Existen otros factores, tales como la disposición microestructural de las trabéculas y sus características que es necesario tener en cuenta para determinar la calidad del hueso y evaluar de manera más directa el riesgo de fractura.

Los avances técnicos de las modalidades de imagen médica, como la tomografía computarizada multidetector (MDCT), la tomografía computarizada periférica cuantitativa (HR-pQCT) y la resonancia magnética (RM) han permitido la adquisición in vivo con resoluciones espaciales elevadas. La estructura del hueso trabecular puede observarse con un buen detalle empleando estas técnicas. En particular, el uso de los equipos de RM de 3 Teslas (T) ha permitido la adquisición con resoluciones espaciales muy altas. Además, el buen contraste entre hueso y médula que proporcionan las imágenes de RM, así como la utilización de radiaciones no ionizantes sitúan a la RM como una técnica muy adecuada para la caracterización in vivo de hueso trabecular en la enfermedad de la osteoporosis.

En la presente tesis se proponen nuevos desarrollos metodológicos para la caracterización morfométrica y mecánica del hueso trabecular en tres dimensiones (3D) y se aplican a adquisiciones de RM de 3T con alta resolución espacial. El análisis morfométrico está compuesto por diferentes algoritmos diseñados para cuantificar la morfología, la complejidad, la topología y los 
parámetros de anisotropía del tejido trabecular. En cuanto a la caracterización mecánica, se desarrollaron nuevos métodos que permiten la simulación automatizada de la estructura del hueso trabecular en condiciones de compresión y el cálculo del módulo de elasticidad.

La metodología desarrollada se ha aplicado a una población de sujetos sanos con el fin de obtener los valores de normalidad del hueso esponjoso. Los algoritmos se han aplicado también a una población de pacientes con osteoporosis con el fin de cuantificar las variaciones de los parámetros en la enfermedad y evaluar las diferencias con los resultados obtenidos en un grupo de sujetos sanos con edad similar.

Por último, se realizó una evaluación de la reproducibilidad de la metodología mediante el cálculo de los parámetros morfométricos y mecánicos de adquisiciones en 5 tibias de oveja repetidas durante tres días consecutivos. Se realizó también un proceso de validación de la cuantificación de los parámetros mediante una comparación de los resultados con los obtenidos tras la aplicación de la metodología a las muestras de tibia adquiridas con microtomografía computarizada $(\mu \mathrm{CT})$, técnica que proporciona una muy elevada resolución espacial.

Los desarrollos metodológicos propuestos y las aplicaciones clínicas proporcionan resultados satisfactorios, presentando los parámetros una alta sensibilidad a variaciones de la estructura trabecular principalmente influenciadas por el sexo y el estado de enfermedad. Por otra parte, los métodos presentan elevada reproducibilidad y precisión en la cuantificación de los valores morfométricos y mecánicos. Estos resultados refuerzan el uso de los parámetros presentados como posibles biomarcadores de imagen en la enfermedad de la osteoporosis. 


\section{Resum}

L'osteoporosi és una malaltia òssia que es manifesta amb una menor densitat òssia i un deteriorament de l'arquitectura de l'os esponjós. Tots dos factors augmenten la fragilitat òssia i el risc de patir fractures, especialment en dones, on hi ha una alta prevalença. El diagnòstic actual de l'osteoporosi consisteix en la quantificació de la densitat mineral òssia (DMO) mitjançant tècniques d'absorciometria dual de raigs $X$ (DXA). Tanmateix, la DMO no es pot considerar de manera aïllada per a l'avaluació del risc de fractura o dels efectes terapèutics. Altres factors, com ara la disposició de les trabècules a nivell microestructural i les seves característiques s'han de tenir en compte per determinar la qualitat de l'os i avaluar de manera més directa el risc de fractura.

Els avenços tècnics de les modalitats d'imatge mèdica, com la tomografia computada multidetector (MDCT), tomografia computada perifèrica quantitativa (HR-pQCT) i la ressonància magnètica (RM) han permès l'adquisició in vivo amb alta resolució espacial. L'estructura de l'os trabecular pot ser observada amb un gran nivell de detall utilitzant aquestes tècniques. En particular, l'ús dels equips de RM de 3 tesles (T) ha permès l'adquisició a resolucions espacials molt elevades. A més, el bon contrast que s'obté entre l'os i la medul la en les imatges de RM, així com la utilització de radiacions no ionitzants fan d'aquesta tècnica una ferramenta idònia per a la caracterització in vivo de l'os trabecular en la malaltia de l'osteoporosi.

En aquesta tesi, els nous desenvolupaments metodològics per a la caracterització de la morfometria i les propietats mecàniques de l'os trabecular en tres dimensions (3D) s'apliquen a adquisicions d'alta resolució provinents de RM de 3T. L'anàlisi morfomètric està composta per diferents algorismes dissenyats per quantificar la morfologia, la complexitat, la topologia i els paràmetres d'anisotropia del teixit trabecular. Quant a la caracterització mecànica, s'han desenvolupat nous mètodes per a la simulació automatitzada 
de l'estructura de l'os trabecular en condicions de compressió i el càlcul del mòdul d'elasticitat.

La metodologia desenvolupada es va aplicar a una població de subjectes sans amb la finalitat d'obtenir els valors de normalitat de l'os esponjós. Els algorismes s'han aplicat també a una població de pacients amb osteoporosi per tal de quantificar les variacions dels paràmetres de la malaltia i avaluar les diferències amb els resultats obtinguts en un grup sa d'edat similar.

Finalment, es va realitzar una avaluació de la reproductibilitat dels mètodes mitjançant el càlcul dels paràmetres de morfometria i de comportament mecànic a partir de les adquisicions de RM de 5 tèbies d'ovella repetides en tres dies consecutius. Es va realitzar a més una validació de les mesures mitjançant la comparació dels resultats de RM amb els obtinguts mitjançant l'aplicació de la metodologia a les mostres de tíbia adquirides amb microtomografia computada $(\mu \mathrm{CT})$, que proporciona una resolució espacial molt elevada.

Els desenvolupaments metodològics proposats i les aplicacions clíniques presentades ofereixen resultats satisfactoris, $\mathrm{amb}$ una alta sensibilitat dels paràmetres a les variacions del teixit trabecular principalment influenciades pel sexe i el grau d'osteoporosi. D'altra banda, els mètodes tenen una alta reproductibilitat i precisió en la quantificació dels paràmetres morfomètrics i mecànics. Aquests resultats reforcen l'ús dels paràmetres morfomètrics i mecànics presentats com a biomarcadors d'imatge en la malaltia de l'osteoporosi. 


\section{Motivation}

Osteoporosis is a bone disorder that supposes a global concern all over the world [1]. This syndrome is characterized by a decreased density of the bone tissue due to abnormal resorption mechanisms and also deterioration in the trabecular bone architecture, both factors induce an increased bone fragility and higher risk of suffering skeletal fractures. All osteoporotic fractures which mostly occur in the hip, vertebrae, wrist and ribs are associated with considerable morbidity, a decline in quality of life, and increased mortality [2]. This disorder is highly prevalent in women, especially in post-menopausal ages, leading to pain and disability associated with fractures [3].

Osteoporosis is associated with decreased bone strength, which is a consequence of the variations present not only in the bone density but also in its quality. Current diagnosis of osteoporosis is largely based on measurement of bone mineral density (BMD), using dual energy X-ray absorptiometry (DXA) techniques. The DXA technique has been shown to be accurate, precise and reproducible in the BMD quantification. However, it has been demonstrated that not only the quantity of bone loss is important but also the degree and nature of the trabeculae atrophy [4]. Therefore, the BMD calculation alone is not sufficiently complete for the whole characterization of trabecular bone deterioration and the estimation of the fracture risk [5]. The architectural abnormalities associated with osteoporosis produce an alteration of specific structural and mechanical parameters of the cancellous bone that should be taken into account and quantified for a more comprehensive approach to the disease and improve in its prevention, diagnosis and treatment therapies.

The significant advances of digital radiology during the last decades, in combination with the application of computational analysis methods to medical images, have allowed for the extraction of relevant medical information and measurements which are not directly appreciable by visual inspection of the images. These measurements can be considered as Imaging Biomarkers, defined 
as all those characteristics that can be extracted and objectively measured from the images of the tissues. Different biological, physiological and chemical biomarkers that characterize organs and tissues can be obtained after the proper postprocessing of medical images. The analysis of these biomarkers can be expressed as virtual biopsies of the organs and their tissues performed by the parametric treatment of images acquired with different modalities. The progress to integrate digital scenarios with multidisciplinary teams dedicated to medical image postprocessing and quantification is transforming medicine and clinical research.

Trabecular bone properties and characterization have been analyzed ex vivo in individual samples by the use of micro-computed tomography $(\mu \mathrm{CT})$ techniques $[6,7]$, which permits spatial resolutions in the range of a few micrometers $(\mu \mathrm{m})$. The high spatial resolutions achievable nowadays in vivo with the new clinical imaging modalities permits the observation of the trabecular bone with good detail in non-invasive and more available ways. Specially, development of digital conventional radiographs, multi detector computed tomography (MDCT), high-resolution peripheral quantitative computed tomography (HR-pQCT) and magnetic resonance imaging (MRI) have allowed the research of in vivo characterization of cancellous bone microarchitecture.

Although CT appears to be particularly suited for investigating the structure of calcified tissues due to the excellent contrast between bone and soft tissues [8], radiation dose limitations and point-spread function (PSF) blurring of X-raybased tomographic methods limit the achievable resolution even at peripheral anatomic sites.

The high inherent contrast between bone and bone marrow, and the higher spatial resolution achievable with current high field systems besides its nonionizing radiations, render MRI as an especially suited technique for the in vivo characterization of trabecular bone microarchitecture [4].

Different morphometric and mechanical parameters can be extracted from MRI acquisitions after appropriate image processing. The parameters included in the 
morphometric characterization include morphology, structural complexity, topology and anisotropy related to the trabecular bone network. The mechanical characterization of the cancellous bone is usually performed by the generation of trabecular bone meshed models and the simulation of different mechanical conditions using the finite element (FE) method. Some of these parameters have been investigated in different and independent studies. However, a validation of the measurements performed with MRI and the definition of normality values in healthy populations have not been yet established. A complete characterization of the mentioned parameters and the analysis of their variations with sex, age and osteoporosis status are necessary in order to integrate the microarchitecture analysis of the cancellous bone into the clinical routine and complement the DXA in the diagnosis and follow-up of the disease.

The development and implementation of the algorithms for a morphometric and mechanical assessment of the cancellous bone from MRI, together with the validation of the measurements and the evaluation of the parameters with factors like age, sex and the osteoporosis status are important research focuses to achieve a complete integration of these techniques into the clinical workflow. This knowledge may improve the diagnosis and treatment of osteoporosis for a more direct approach to estimate the fracture risk. 


\section{Objectives and structure}

This thesis focuses on a complete in vivo morphometric and mechanical characterization of the trabecular bone, from MR acquisitions at high spatial resolution, for the assessment of osteoporosis.

Particularly, the following objectives are proposed:

- To develop and implement proper image processing algorithms for an efficient preparation of the data.

- To develop and implement algorithms for a morphometric analysis of the trabecular bone and extract characteristic parameters of:
o Morphology
o Complexity
o Topology
o Anisotropy

- To develop and implement algorithms for a voxel based FE model generation of the trabecular bone that can be directly imported from FE commercial software.

- To perform compression simulations of the trabecular bone in order to calculate stresses, strains and elastic modulus of the tissue.

- To define normality ranks for the extracted parameters by the analysis of a healthy population.

- To evaluate the alterations observed in the parameters in a group of patients with osteoporosis.

- To validate the methodology by the application of the algorithms to 5 MRI acquisitions of sheep tibiae performed at 3 Tesla ( $\mathrm{T}$ ) and compare the results of the application to the same samples acquired using $\mu \mathrm{CT}$.

- To integrate all the methodology in a software tool for the automated calculation of the trabecular bone morphometric and mechanical properties in the clinical routine. 
The thesis is structured as follows:

Chapter 1 is an introduction which describes the main concepts that appear in the thesis. First, the clinical scenario of osteoporosis and its consequences on the bone tissue are detailed. Second, MRI technique is reviewed and specific sequences for the analysis of trabecular bone are commented. And finally the different morphometric and mechanical parameters of the trabecular bone are introduced, summarizing the methods and the state of the art.

Chapter 2 describes the thesis methodology. It is divided into five parts: Image acquisition, where specific details about acquisition protocol and MRI parameters are commented; image processing, with the different steps for the preparation of the data before image analysis; morphometric analysis, where different methods for quantifying morphology, complexity, topology and anisotropy are detailed; mechanical analysis, with the description of the FE model generation and its mechanical analysis using the FEM; validation, where the processes followed for the evaluation of reproducibility of MRI measurements are detailed, and the sample preparation and $\mu \mathrm{CT}$ acquisition characteristics are specified.

In chapter 3 the results of the application of the methodology to a healthy population are shown, and the influence of osteoporosis status in the parameters is analyzed. Validation results are exposed, including reproducibility of MRI measurements. The discussion on the results is also presented.

Chapter 4 copes with the main conclusions of the thesis and Chapter 5 summarizes the possible lines of future work.

An appendix is included at the end with a brief description of the developed software and its practical integration in the clinical routine. 


\section{Introduction}

In this chapter, the main concepts and technologies involved in the development of the thesis are introduced. It is divided in three main sections, the first deals with the clinical background of the investigation, with special interest in the bone tissue properties and changes with the osteoporosis disease. Secondly, a description of the MRI technique from its physic principles to the imaging requirements for the study of trabecular bone is given. Finally, the methods for the image analysis applied to trabecular bone and the quantification of bone properties are introduced.

\subsection{Clinical scenario: Osteoporosis}

The present part is divided into three subsections explaining the bone tissue properties, including anatomy and function and a review of the bone development and remodeling processes. A special focus in the dynamics and evolution of the bone structure and its relationship with metabolism alterations in osteoporosis disease is specified.

\subsubsection{The bone tissue}

Bone has an important combination of physical properties, showing a very high resistance to traction and compression [9]. At the same time it presents certain elasticity and the advantage of being a relatively light material. At all organization levels, from the macroscopic to the submicroscopic structure of bones, their constitution ensures the maximum resistance with reduced material and the lowest weight.

At the structural level, two different forms of bone can be distinguished in general: cortical or compact bone, which, for example, forms the midshaft (or diaphysis) of long bones, thus providing protection for the medullary cavity, 
and cancellous, trabecular, or spongy bone, which in long bones is found mainly at their ends (Figure 1.1). The ends of long bones are the metaphysis and epiphysis and consist mainly of spongy bone covered with a thin crust of compact bone. In typical long bones such as femur, humerus or radius, the shaft is a thick-walled cylinder made of compact bone, with a voluminous central cavity occupied by the bone marrow.

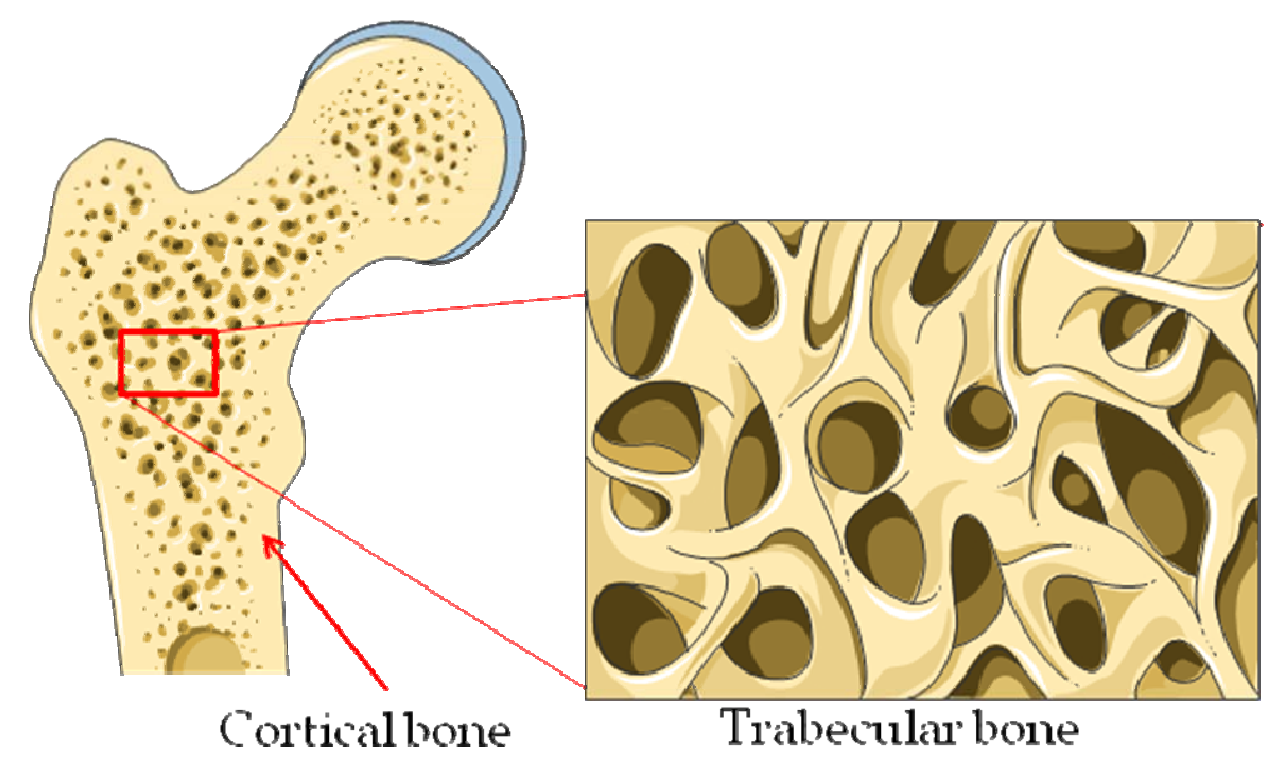

Figure 1.1 Iconography and detail of main bone structural organizations in a femur. Cortical bone (left) and trabecular bone (zoomed, right). From [10].

Cancellous bone is compound by a three-dimensional (3D) reticulum of branched bone spicles, known as trabeculae, which delimit a labyrinthine system of interconnected spaces, or pores, occupied by the bone marrow. The high surface-to-volume ratio of trabecular bone is necessary for its metabolic function, whereas cortical bone has mainly a mechanical and protective function [11].

The periosteum is the external surface of bones and is in contact with the surrounding soft tissue. The endosteum is the internal surface facing the medullary cavity. Both the periosteum and the endosteum are organized structures made up with connective tissue distributed in layers of chondrogenic and osteogenic cells $[12,13]$. 
Trabecular bone and cortical bone are mainly composed of the same microstructural elements: organic matrix, crystalline inorganic matrix, cells, and soluble factors.

Bone cells form a small part of the total bone mass. Compact bone interstitial substance consists mainly of cortical mineralized bone matrix, deposited in layers or lamellae. The lamellae are arranged in three different ways although the vast majority is distributed concentrically around vessels within the bone forming the Haversian systems, which are cylindrical structural units. The Haversian canals are communicated with the surface or the interior cavity and with each other by transverse or oblique channels called Volkmann's canals (Figure 1.2).

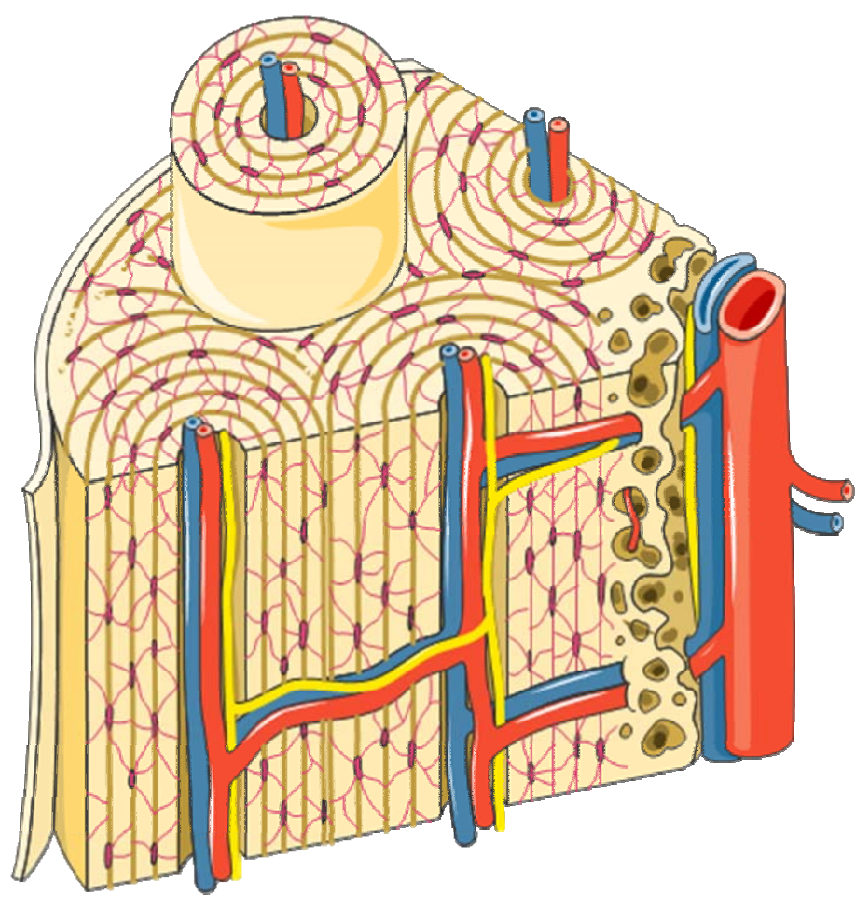

Figure 1.2 Detailed section of cortical bone where Haversian canals in the longitudinal direction can be appreciated. Volkmann's canals are interconnecting Haversian canals with the endosteum. From [10].

Spongy bone is also composed of lamellae, but the trabeculae are relatively thin (80 to $150 \mu \mathrm{m}$ ) and usually do not contain blood vessels inside [9]. Therefore, no Haversian systems exist and a complex grouping of angular pieces of lamellar bone form the cancellous bone. The bone cells are nourished by diffusion from the endosteal surface. 
The interstitial substance of bone is formed by two main components, an organic matrix which represents the $35 \%$, and various inorganic salts which comprise $65 \%$ of its dry weight. The organic substance of bone matrix is formed by $90 \%$ of collagen type I, which fibers have a mean diameter of 50 to $70 \mathrm{~nm}$.

The inorganic substance of bone is composed of submicroscopic deposits of a type of calcium phosphate very similar to hydroxyapatite. The calcium phosphate is present as crystals. The crystals are not randomly distributed but appear regularly at intervals of 60 to $70 \mathrm{~nm}$, along the fiber [9].

During development and growth, the amount of organic material per unit volume remains relatively constant, but the amount of water decreases and the proportion of bone minerals increases, which reach a maximum of about $65 \%$ of fat-free dry weight in tissue of adults.

When bone is subjected to the action of a weak acid or a chelating agent, inorganic salts are removed. The demineralized bone thus loses most of its hardness, but is still quite strong and flexible. It retains its macroscopic form and the normal microscopic appearance. On the other hand, when the organic constituents of bone are extracted, the inorganic residual components have the macroscopic shape of bone and the microscopic topography is also maintained to some extent. However, the bone has lost most of the tensile strength it had, and is now as fragile as porcelain.

One can thus conclude that the hardness of bones depends on their inorganic components, while their strength and elasticity depend on their organic matrix, particularly collagen. Without one of these two components, bone and skeletal tissue would be a material with very poor characteristics, but with both components it is a neat and remarkably durable tissue, superbly adapted to their mechanical function.

\subsubsection{Bone development and remodeling}

Nature varies the stiffness of bone material by modulating the concentration of the crystals of hydroxyapatite-like mineral in the collagen matrix. Higher mineral content increases stiffness but sacrifices flexibility. If bone is 
undermineralized (due to high remodeling states), it becomes too flexible and will bend "too much" in loading and crack; if overmineralized (if remodeling is greatly suppressed), it will bend too little and crack [3].

Despite of its strength and hardness, bone is a dynamic and live material which is being continuously renewed and that experiments a permanent remodeling during the whole life of the subject [11]. Thus, even after the growth phase has ended, the skeleton is continuously regenerating and is completely renewed about every 10 years while preserving its structural anatomy and function. The combination of bone-resorbing osteoclasts and bone-forming osteoblasts acting in a coordinated way is the responsible for the bone remodeling process.

Osteoclasts and osteoblasts are well differentiated cells which work together in a unique spatial structure called the basic multicellular unit (BMU) [14]. An histological image of a BMU can be appreciated in figure 1.3.

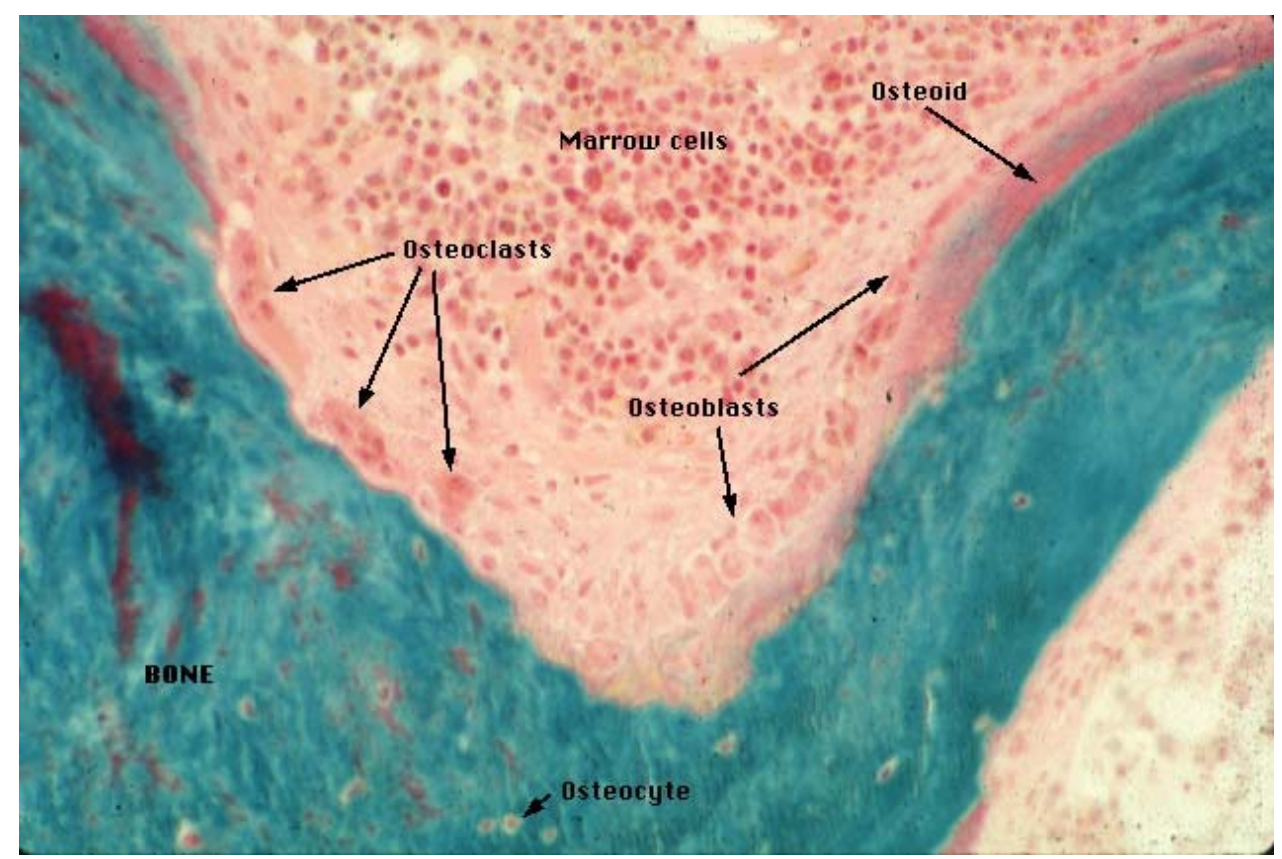

Figure 1.3. Histological section showing the components of a basic multicellular unit (BMU). From [15].

Inside each BMU, preosteoblasts move into the areas previously dug by osteoclasts, and while differentiating to mature osteoblasts, refill the remaining resorption cavities by secreting several specific proteins which develop later into the mineralization of the osteoid. The life of BMU is much longer than the 
life of osteoblasts and osteoclasts. Thus, older cells of both types have to be replaced by new ones that have to be recruited from their respective precursors and directed to the bone-remodeling sites $[16,17]$. This relationship between formation and resorption of bone is known as the bone balance, and it can be positive, as occurs when bone formation is superior to bone resorption, or negative, when osteoclasts resorb more bone than osteoblasts can produce.

The progenitors of the osteoblasts are located within the bone marrow and are exposed to a variety of systemic hormones and local factors. Osteoblasts have a mesenchymal origin, while osteoclasts derive from undifferentiated hematopoietic cells. It must be noted that osteoclast production is strictly dependent on close contact with stromal cells and osteoblasts $[18,19]$.

In the particular case in which rapid formation of new bone is mandatory, such as in periods of fracture curation, in skeletal growth in early childhood, or in particular metabolic bone disorders, instead of lamellar bone, a provisional distribution of bone is formed, in which the collagen fibers are randomly oriented. Under physiologic conditions, this provisional bone is replaced after some time by lamellar bone, which has better mechanical properties [12,13].

After menopause, increased remodeling with a more negative bone balance in the many BMUs removes more bone more rapidly from an ever-diminishing and architecturally disrupted bone.

\subsubsection{Osteoporosis}

Osteoporosis is a multifactorial disorder which affects the entire skeleton and is characterized by a low bone mass in combination with a loss of quality of the trabecular organization causing microarchitectural changes. As a consequence of the increased bone fragility, an increased risk of fractures exists. The bone loss occurs slowly and 'silent'. In fact, in most cases the first symptom is a fracture. The majority of bone fractures associated with osteoporosis take place in vertebrae, hip or wrist (Figure 1.4) [20]. 


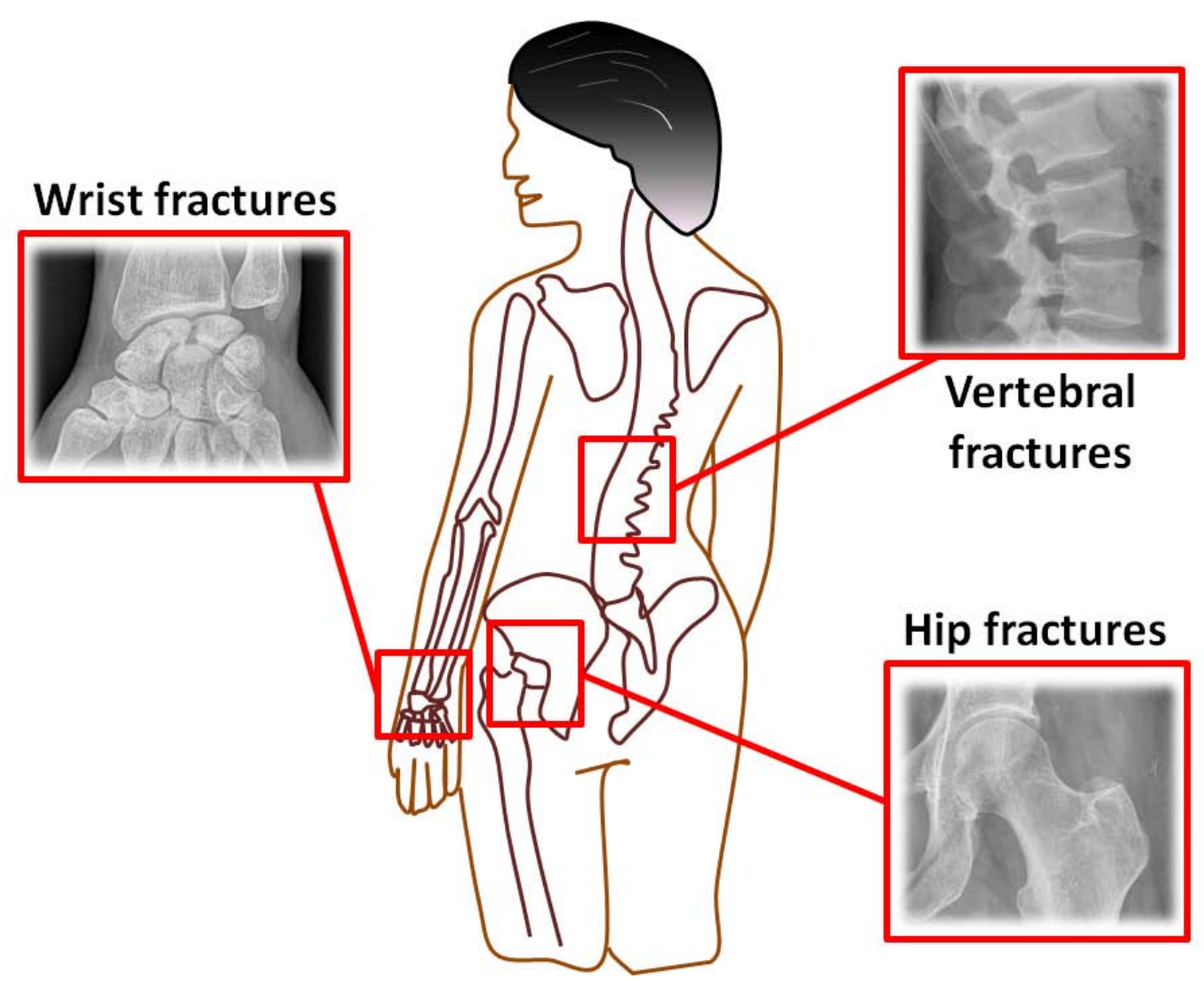

Figure 1.4. Common fractures in osteoporosis.

Osteoporosis is the most prevalent metabolic bone disease in developed countries and supposes a global concern affecting nearly 200 million people worldwide [1].

The prevalence of osteoporotic fractures increases with age. Osteoporosis is prevalent in women, especially in post-menopausal ages, leading to pain and disability associated with bone fractures [1]. It is estimated that over the age of 50 years, 1 in 3 women and 1 in 12 men worldwide have osteoporosis [21]. In the European Union, in the year 2000, the number of osteoporotic fractures was estimated at 3.79 million, of which 0.89 million were hip fractures [22]. Of all fractures due to osteoporosis, hip fractures are the ones that are most disabling. Bone fractures constitute a major public health and healthcare challenge [23]. In 1990, about 1.7 million new hip fractures occurred worldwide, and this figure is expected to rise up to 2.6 million by 2025 [24]. Furthermore, women who have sustained a hip fracture have a $10 \%$ to $20 \%$ higher mortality than would be expected for their age [25]. 
Current diagnosis of osteoporosis is largely based on measurement of BMD, using DXA of the hip or lumbar spine. An example of a clinical DXA system can be appreciated in figure 1.5. An individual BMD value compared with the mean of a healthy young population in terms of the number of standard deviations (SD) is termed the T-score. The World Health Organization (WHO) has defined osteoporosis as a T-score of less than -2.5 SD and osteopenia as a T-score between -1 and -2.5 SD [26]. The diagnosis criteria and staging of osteoporosis according to the WHO guidelines can be observed in Table 1.1.

\begin{tabular}{|c|c|}
\hline Stage & DXA T-score \\
\hline Normal & $>-1.00 \mathrm{SD}$ \\
\hline Osteopenia & Between $-2.50 \mathrm{SD}$ and $-1.00 \mathrm{SD}$ \\
\hline Osteoporosis & $<-2.50 \mathrm{SD}$ \\
\hline
\end{tabular}

Table 1.1. Diagnosis criteria for osteoporosis based on the T-score resulting from the DXA scan following WHO guidelines.

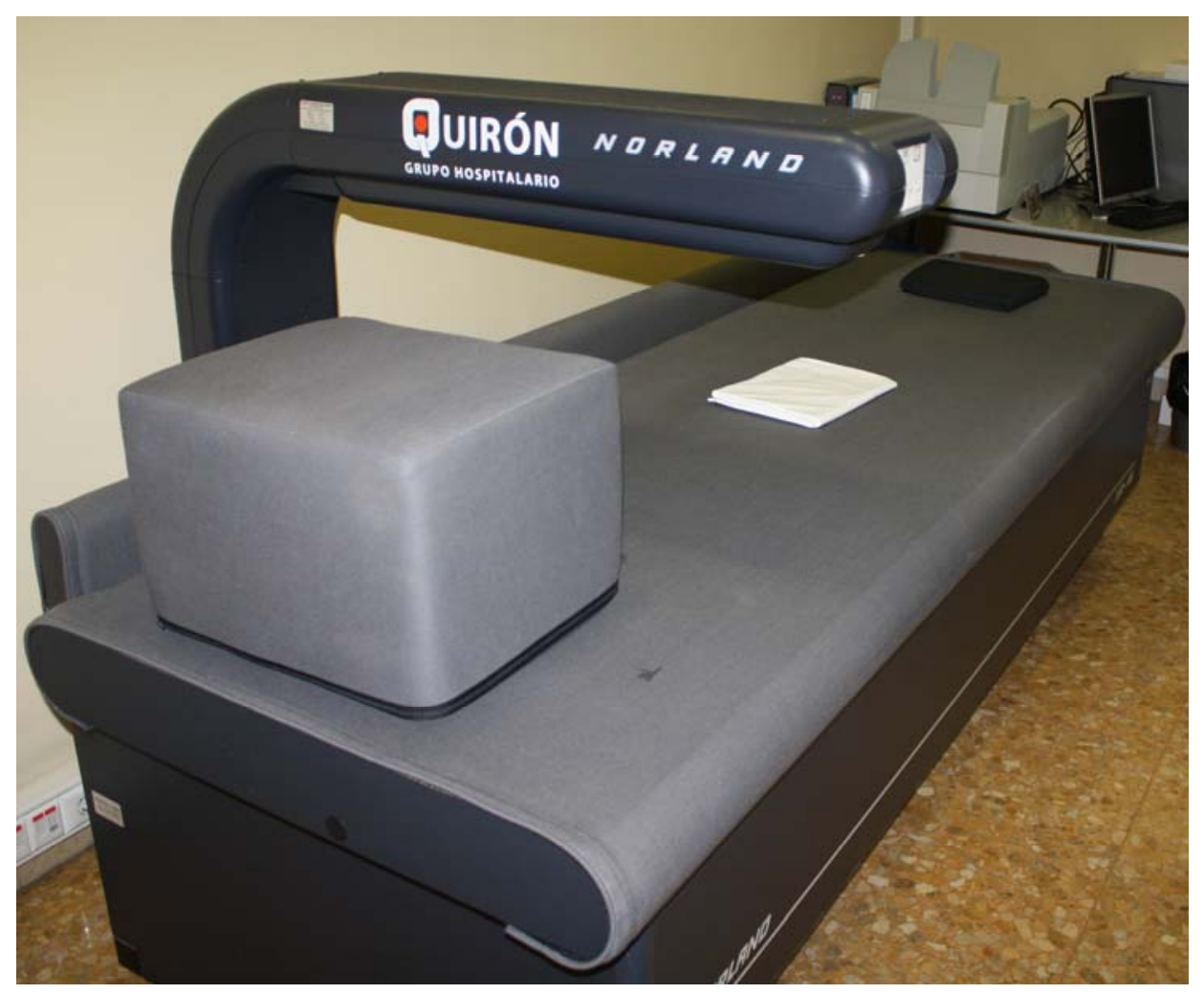

Figure 1.5. Dual-Emission X-Ray Absorptiometry (DXA) scanner (Norland, Fort Atkinson, WI) for Bone Mineral Density (BMD) quantification at Quirón Valencia Hospital. 
Despite the shown conditions for the diagnosis of osteoporosis, it is known that the BMD and age are not the only factors that affect the frequency of fractures [1-4]. The increased remodeling and negative bone balance produce bone loss, trabecular thinning and loss of connectivity, cortical thinning, and porosity. Older, more mineralized interstitial bone, distant from surface remodeling, accumulates microdamage, whereas more superficial bone is replaced with younger less mineralized bone, reducing stiffness [27]. A scanning electron micrograph (SEM) image showing the differences between a normal and an osteoporotic bone can be observed in figure 1.6.

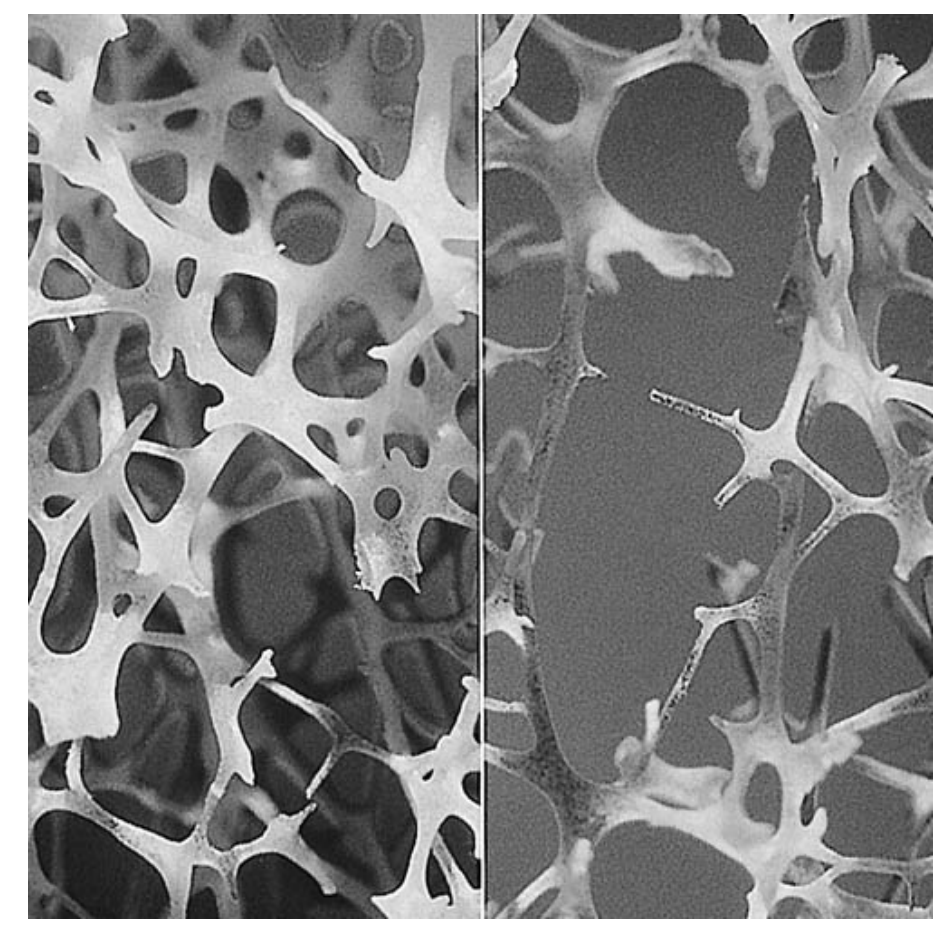

Figure 1.6. Scanning electron micrograph of a normal bone (left) vs. osteoporotic bone (right). From [21].

The microarchitecture descriptors to provide complementary information to BMD are gaining interest as determinants of the bone's quality [28-30]. Although it is accepted that low BMD increases fracture risk, not all patients who are osteoporotic will sustain a fracture. Low BMD as a risk factor for fracture can be compared to high cholesterol as a risk factor for heart disease, being BMD a rather poor predictor of fracture risk [4]. In this sense, an analysis 
performed by Wehrli et al. showed that, on the average, BMD explains about $60 \%$ of bone strength [31].

Regarding available therapies, the pharmacological agents used in the treatment of osteoporosis include antiresorptive agents such as biphosphonates, selective estrogen receptor modulators (SERM), raloxifene, and calcitonin; boneforming agents such as fluoride and parathyroid hormone; and, more recently, a new chemical entity, strontium ranelate, which both increases bone formation and decreases bone resorption. Despite the evidence for efficacy of these treatments for osteoporosis, intervention is not optimal, even in women with a history of fracture [1].

For a more correct assessment of osteoporosis, the nature of bone loss must be deeply investigated in order to determine its influence on bone strength. As an example, given the same fractional reduction in bone volume, a mechanism causing uniform thinning compromises strength less than one disrupting the entire structural elements by an inhomogeneous erosion process [32]. Therefore, the investigation in new methods to characterize trabecular bone microarchitecture alterations produced by the disease is crucial. Such advances may not only be useful for a better diagnosis of the disease, but also to evaluate the efficacy of the existing therapies.

\subsection{MRI}

MRI is a medical imaging technique that serves to explore in vivo detailed inner anatomy and function of organs and tissues within the body. The human body is mainly composed of water and lipids, molecular structures containing a large proportion of hydrogen. Under an intense magnetic field, the hydrogen protons of the body start to precess at a certain frequency. If electromagnetic waves are produced at the proton precession or resonance frequency, the particles absorb

the energy and liberate it to the medium generating a signal which is used in combination with other auxiliary magnetic fields to form the image [33]. MRI technique can produce images with excellent contrast between soft tissues and 
high spatial resolution in every direction. The electromagnetic waves used have low energy and appear to be safe under normal operating conditions [34].

\subsubsection{Physical principles}

\section{Nuclei properties and magnetization}

At the core of atoms, and accommodating most of the elemental mass, is the nucleus, consisting of neutrons and protons. Nuclei with an odd number of protons and neutrons (i.e. $1 \mathrm{H}, 13 \mathrm{C}, 19 \mathrm{~F}, 23 \mathrm{Na}, 31 \mathrm{P}$ ) possess spin-angular momentum. These nuclei have a magnetic moment, $\mu$, characterizing the magnetic field surrounding the nucleus. The magnetic field attributed to a nucleus is analogous to that from a magnetic dipole (figure 1.7).
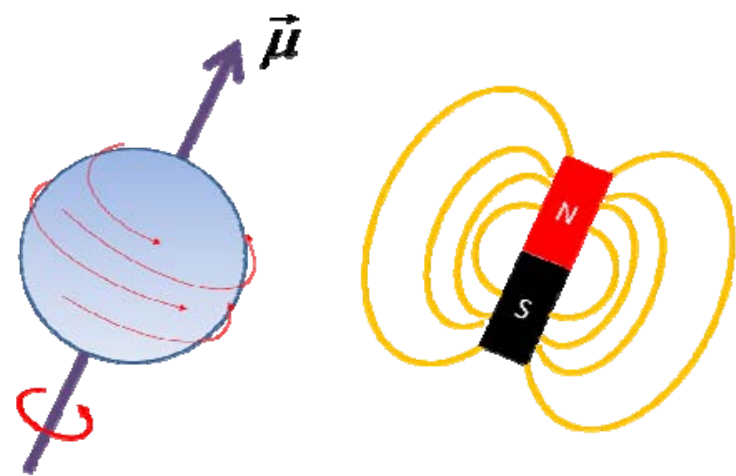

Figure 1.7. Nuclear magnetism. Nuclei with net spin have a characteristic magnetic moment $(\vec{\mu})$ and an associated magnetic field similar to a dipole.

Unfortunately, atoms with its nuclei formed by an even number of protons and neutrons, like calcium $(40 \mathrm{Ca})$, which is also abundant in the human body, do not have a spin-angular momentum and do not produce an MR signal.

When exposed to a magnetic field, the randomly oriented magnetic dipoles tend to align with the magnetic field. This phenomenon is explained by quantum mechanics. Due to the spin characteristics of hydrogen, under a magnetic field $B_{0}$, protons distribute in two energy states, either parallel or antiparallel to the main magnetic field, being the last the state the one with more energy. 
The net magnetization is the vector sum of individual magnetic moments. If the protons are not under the effect of a magnetic field, they are aligned randomly and the net magnetization in a given unit of volume is zero. However, under the effect of a magnetic field, protons tend to align with the $B_{0}$ direction (figure 1.8). Even more, the major part of protons are aligned in the parallel orientation. The total magnetization of the parallel orientation minus the total magnetization of the antiparallel results in a non zero and positive net magnetization $\vec{M}_{0}$.

The net magnetization experiences a torque from the main magnetic field $B_{0}$. As a result, the magnetization precesses around the axis of the magnetic field at a special frequency called the Larmor frequency, $f_{L}$ :

$$
f_{L}=\gamma \cdot B_{0}
$$

where $\gamma$ is the gyromagnetic ratio (for hydrogen $\gamma_{H}=42.58 \mathrm{MHz} / \mathrm{T}$ ) and $\mathrm{B}_{0}$ is the static magnetic field. If the main magnetic field is a common $1.5 \mathrm{~T}$ system, the theoretical precession frequency of protons is $63.87 \mathrm{MHz}$ while $127.74 \mathrm{MHz}$ for a 3 T system.

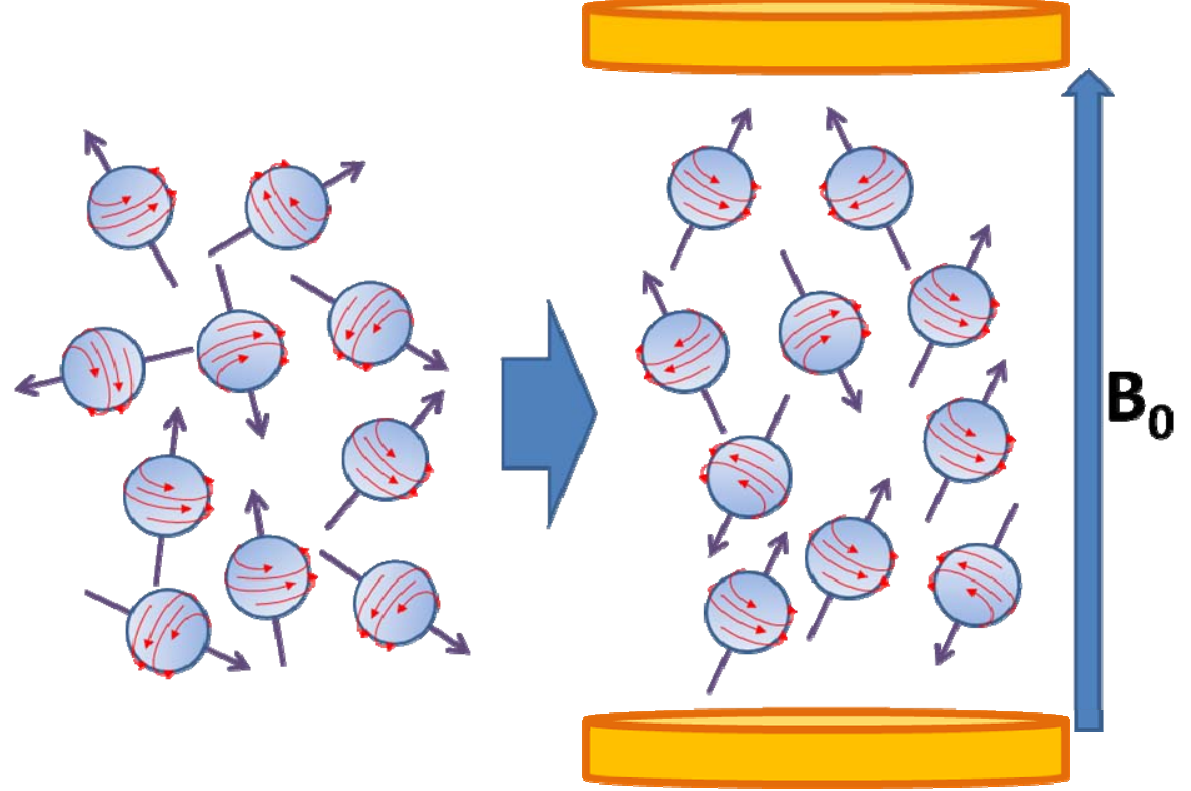

Figure 1.8. Protons alignment in the direction of the main magnetic field $B_{0}$. When no static field is present, random orientations exist for the magnetization vector. Under a static magnetic field, protons are aligned and have a precession movement which frequency is calculated from the Larmor equation. 
In the situation shown in figure 1.8, a coordinate system must be defined in order to understand excitation and relaxation mechanisms. The direction of the main magnetic field $\left(B_{0}\right)$ is called the longitudinal direction and corresponds to the ' $z$ ' axis of our coordinate system. The plane which is perpendicular to the longitudinal direction is called the transverse plane or the $x-y$ plane.

Excitation and relaxation

In equilibrium conditions, that is, with the unique presence of the static magnetic field $\mathrm{B}_{0}$, there is a positive magnetic moment along the longitudinal axis, $M_{z}>0$. Although protons are precessing and a component of magnetization may exist in the transverse plane, the phase differences makes $M_{x y}=0$.

If an external radiofrequency (RF) pulse is applied at the resonance frequency (Larmor frequency) in a perpendicular direction to $\vec{M}_{0}$, producing an additional magnetic field $B_{1}$, net magnetization can be tipped completely into the transverse plane if the RF pulse is strong enough to produce such a $90^{\circ}$ deviation.

After the application of the RF pulse, the spins are precessing in the transverse $x-y$ plane. If there is a current loop in a plane parallel to the $x-z$ or $y-z$ plane the changing magnetic flux in the loop induces a current in the loop. This current will die out after some time and the measured signal is called the free induction decay (FID) [35]. Immediately after the excitation pulse all spins precess in the same phase. After some time they start to loose their phase coherence due to two effects. First, other spins and the molecular magnetic fields, due to the macro-molecules in the tissue, perturb the total magnetic field experienced by the different free water protons during their fast random motion through these fields. This makes the precession frequency to be slightly different in every proton depending on the specific environment, resulting in dephasing of the proton spins and thus in a decay of the total magnetization. This is called "T2 decay". Second, the "macroscopic" magnetic field in the tissue, which is mainly constant on the local environment of protons is not exactly homogeneous, due to susceptibility variations. The total vector sum of the magnetic moments 
within a volume element considered decreases and so the total magnetization and the induced signal decrease. The total decay caused by both effects is called "T2* decay" and is two to three times faster than pure $\mathrm{T} 2$ decay.

As mentioned, RF energy causes the nuclei to absorb energy exciting them to the transverse plane. The nuclei can return to their original state only by dissipating their excess of energy to their surroundings, which is also known as the lattice. The process, which is called spin-lattice relaxation, describes the recovery of the longitudinal magnetization towards its equilibrium value. The duration of the recovery process is given by the $\mathrm{T} 1$.

The FID signal is not usually measured in MRI applications, since measurements can start only some time after the effective excitation time. In order to control the signal and form images we need to generate echoes of the FID. There are two principal manners of generating an echo in MRI, which define the two principal types of sequences: spin-echo sequences and gradientecho sequences, which will be further explained in following sections.

\subsubsection{System architecture}

\section{Main magnet}

In superconductive MR units, the static magnetic field comes from a large electric current flowing through niobium-titanium wires that are formed into a big loop. A typical clinical superconductive MR system has a magnetic field strength of $1.5 \mathrm{~T}$ or $3 \mathrm{~T}$. These systems can achieve a very strong and stable magnetic field since the wires are immersed in liquid helium at $-268^{\circ} \mathrm{C}$, and large currents can be used due to the superconductivity and the absence of energy heat dissipation. A power supply is needed at installation to inject electric current into the coils of wire in order to generate a magnetic field by circulation. Once the desired and designed field strength is achieved, the power supply can then be removed. The wires can retain this electric current for many years, with no need to inject additional electric current. There exists a minimal loss in electric current and a minimal decrease in the magnetic field strength. 
The liquid helium levels in the magnet will need to be filled at regular intervals to maintain the superconductive properties of the coils [36].

The design of the main magnet is crucial since it must produce an homogeneous magnetic field in a defined region at the center of the coils called the isocenter. There exist different architectures for clinical MR scanners, although the cylindrical is the most extended one due to its field uniformity and stability (figure 1.9).

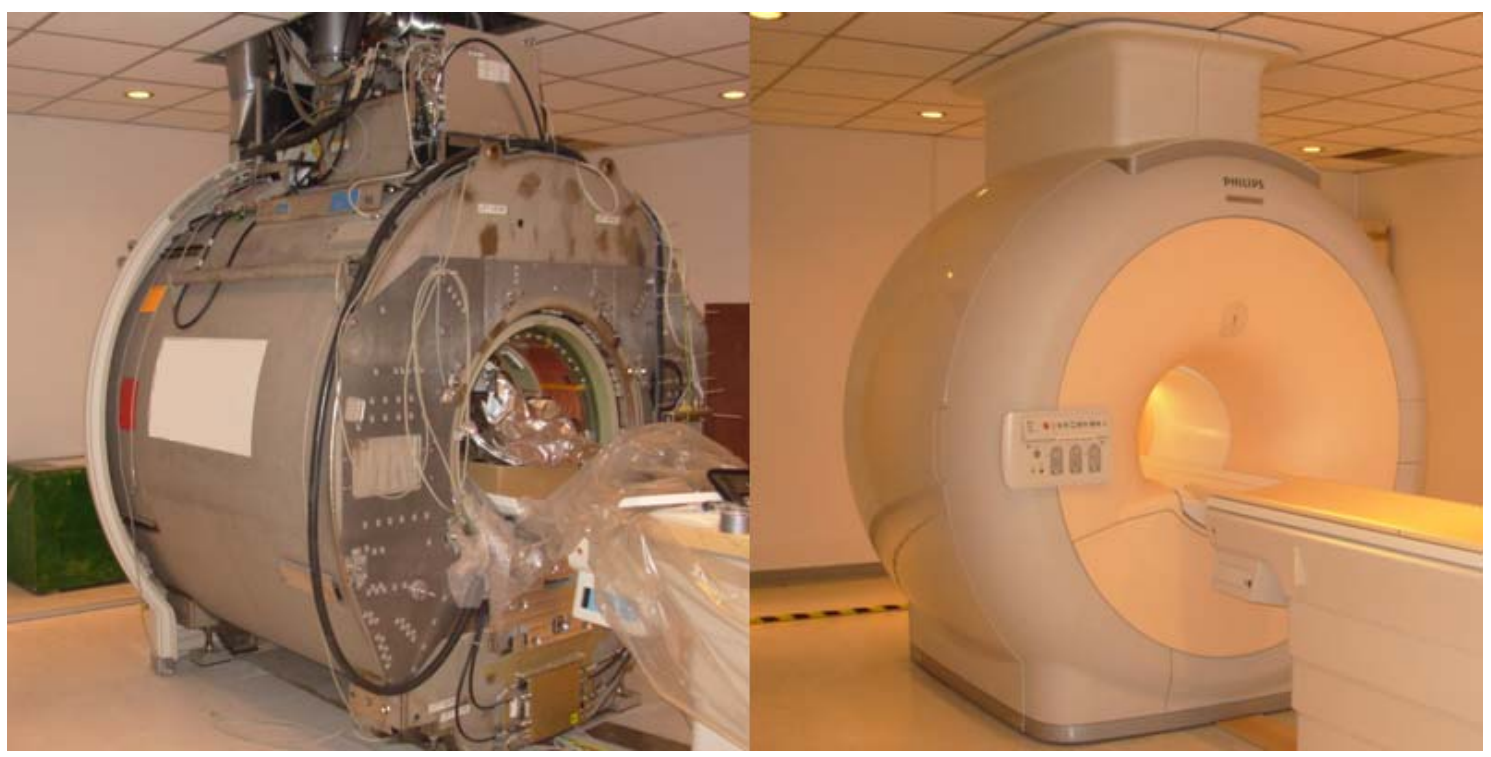

Figure 1.9. MR $3 \mathrm{~T}$ clinical scanner from Philips Healthcare $\odot$. A detail of the main magnet compartment can be appreciated on the left. Final aspect of a cylindrical MR scanner after fixing the external housing.

The field strength that can be achieved with a magnet has a direct influence on the signal-to-noise ratio (SNR) of the resultant MR images. The use of high magnetic fields, like $3 \mathrm{~T}$, permits the acquisitions with a high SNR. This increase in SNR eases the configuration of acquisitions with high spatial resolutions in reduced acquisition times.

\section{The gradient chain}

One of the great advantages of MRI as an imaging method is the possibility of obtaining a tomographic image in any spatial orientation. Gradients are small magnetic fields superimposed on the static field $B_{0}$ that vary linearly with distance and are used for spatial localization. The use of three coils placed in $x$, 
$y$ and $z$ permit the slight spatial variation of the magnetic field in the three directions of space (figure 1.10).

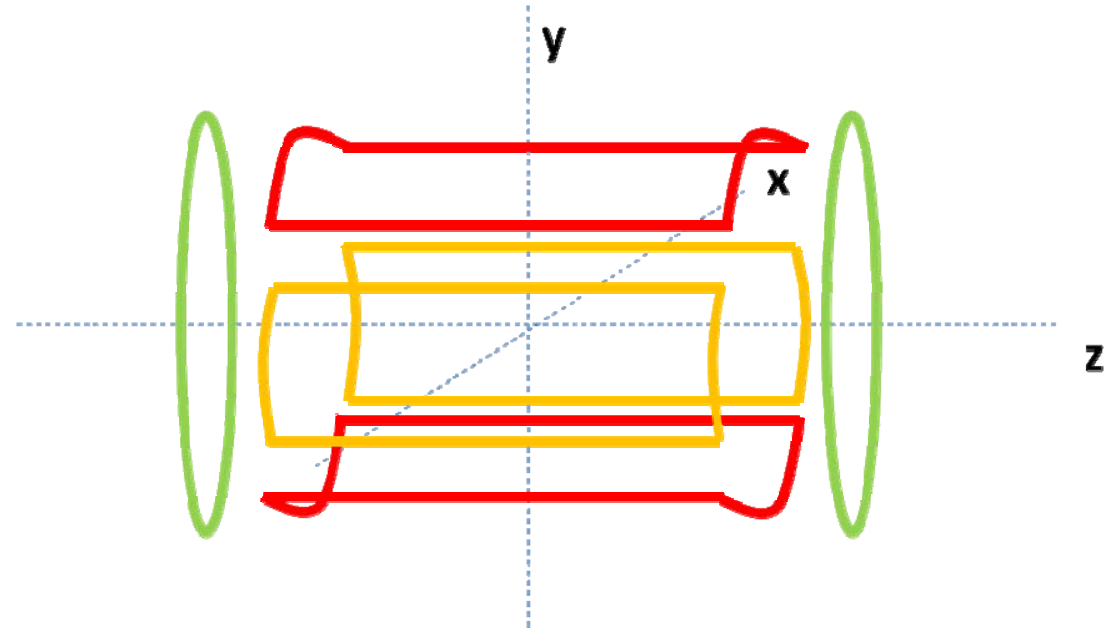

Figure 1.10. Diagram of the gradient coils distribution used for spatial variation of the proton precession frequencies in a cylindrical MR system.

If the magnetic field is uniform inside the magnet all nuclei may absorb the RF energy at the same frequency. To achieve a selective excitation of a plane, it must be established a uniform magnetic field variation along the direction perpendicular to the plane chosen, which is a magnetic gradient in that direction. Field variations produced with the gradients are expressed in $\mathrm{mT} / \mathrm{m}$. The characteristics of the gradient system determine the resolution and velocity of acquisition. For a gradient with reduced activation time and high amplitude, faster acquisition times and high spatial resolutions can be obtained in the MR examinations.

\section{The RF chain}

The RF system is responsible for generating and broadcasting the RF energy $\left(B_{1}\right)$ used to excite the spins. It contains four main components: a frequency synthesizer, a digital envelope of RF frequencies, a high power amplifier, and a coil or antenna. Each RF pulse that is broadcast to the patient consists of two parts: a center or carrier frequency and a discrete envelope or function containing a range or bandwidth of frequencies. The frequency synthesizer 
produces the center or carrier frequency for the pulse. The specific frequency is generated as a phase coherent signal. It is mixed with the RF envelope prior to amplification. The RF power amplifier is responsible for producing sufficient power from the frequency synthesizer signal to excite the spins. The amplifier may be solid state or tube type. The actual amount of power required from the amplifier to rotate the spins from equilibrium depends on the specific frequency, coil transmission efficiency, transmitter pulse duration, and desired excitation angle. The final component of the RF system is the transmitter coil. All MR measurements require a transmitter coil or antenna to broadcast the RF signals [37].

\section{Data acquisition system}

The data acquisition system is responsible for measuring the signals from the spins and digitizing them for later postprocessing. All MRI systems use receiver coils to detect the induced voltage from the spins following an RF pulse. The exact shape and size of the coil is manufacturer specific, but its effective field, like that of the transmitter coil, must be perpendicular to $B_{0}$ [37].

The signals produced by the spins are usually $n \mathrm{~V}-\mu \mathrm{V}$ in amplitude and $\mathrm{MHz}$ in frequency. The signal needs to be amplified and demodulated to kilohertz $(\mathrm{kHz})$ frequency, filtered using a low pass filter and digitized by the analog-todigital converters (ADC). Digitized signals are temporally stored in a matrix of spatial frequencies called the $k$-space for further operations and generation of the images.

\section{Computer system}

Every MR system has a minimum of two computers. One controls the user interface, the other is an array processor which consists of a dedicated computer system for performing the multidimensional Fourier transformations to the detected raw data and form the final images [37]. In the case of 3D acquisitions as the used in the current investigation, a 3D space of frequencies is acquired and high computation memory and efficiency is needed in order to perform the 
Fourier transformation to the whole volume and obtain the desired plane partitions or images.

\subsubsection{Pulse sequences}

The design of appropriate RF pulse sequences is a very important matter in the advancement of MRI. Apart from obtaining different image weightings on the same tomographic plane and improve the radiologist differential diagnosis process, its interest lies in the possibility of making scan times shorter and shorter. This has led to the development of many pulse sequences with several configurations and different names according to the different manufacturers. However, there are two sequences which are considered the basis of the rest of MR pulse sequences, since they are defined directly from the two existing ways for generating an echo of the FID signal, that is, using the RF system in the SpinEcho sequences (SE) or using the gradient system in the gradient-echo sequences (GRE).

\section{$\underline{\text { SE sequences }}$}

The classic SE sequence consists of an initial $90^{\circ}$ RF pulse which excites the nuclei and tips the magnetization to the transverse plane. At the end of excitation, all the nuclei have exactly the same phase, and a high positive magnetization is present in the $x-y$ plane. Immediately after the application of the excitation pulse, the nuclei start to relax for some time (TE/2) while losing their phase coherence. Then, a second RF pulse of 180 degrees is sent and the position of the spins on the magnetic field is reversed. Nuclei start recovering

the phase coherence and an echo signal is formed. The process is repeated every TR seconds. A diagram of the SE pulse sequence can be appreciated in figure 1.11 . 


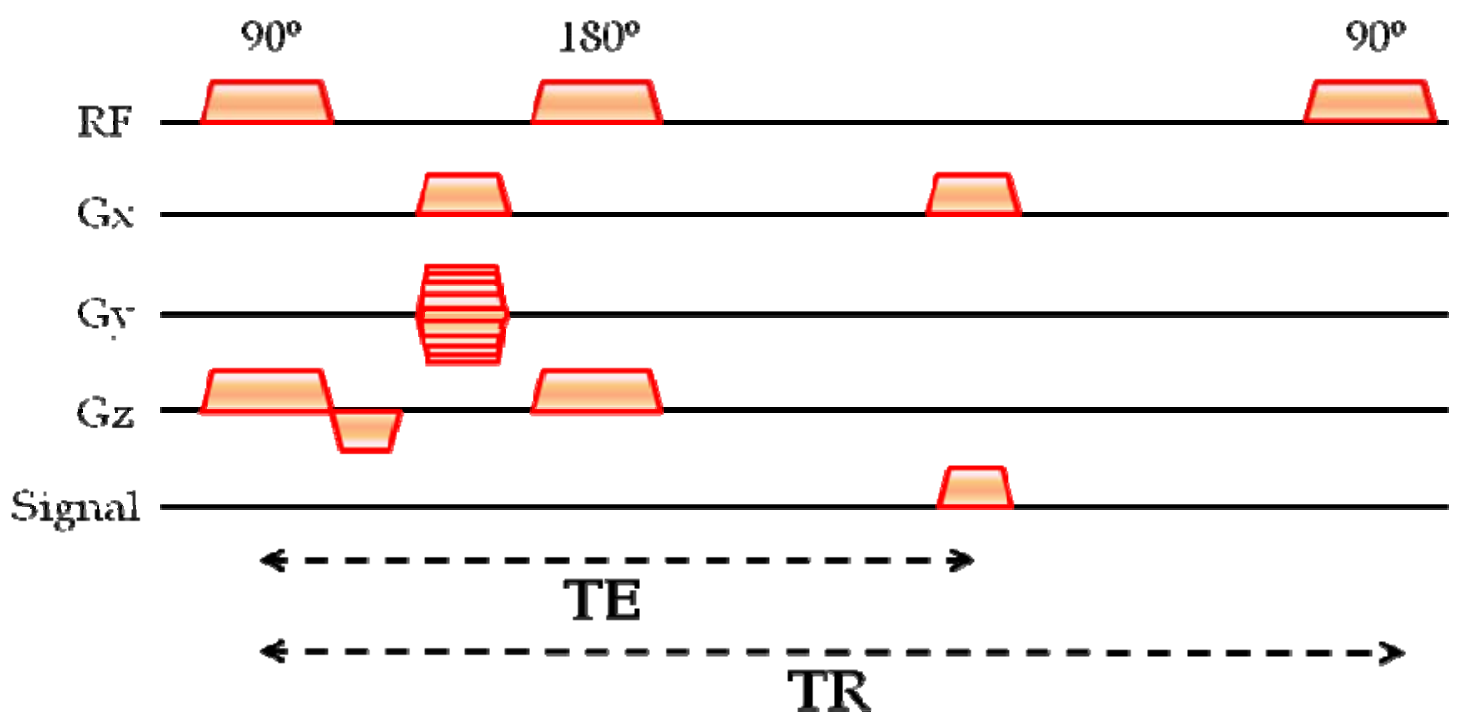

Figure 1.11. Spin-echo sequence pulse diagram

The sequence diagram is showing the RF pulse activations together with the gradients for slice selection step in excitation $(G z)$, and frequency and phase encoding during relaxation ( $G x$ and $G y$, respectively).

\section{GRE sequences}

In the conventional SE sequence, typical acquisition times are of the order of minutes since the $90^{\circ}$ and the $180^{\circ}$ pulses take time and the magnetization must be fully recovered between each TR. The reduction of MR acquisition times with modern acceleration techniques is actually a key research subject in different groups around the world. As a preliminary idea for acceleration of the SE sequences, shorter TR times could be used, however this would involve contrast limitation problems due to the TR reduction and images would tend to be T1 weighted. Another solution could consist in replacing the initial $90^{\circ}$ pulse by a pulse of lower amplitude $<90^{\circ}$, allowing for a faster recovery. Also, another possibility would consist in removing the $180^{\circ}$ pulse, however, an added solution must be found for the echo generation.

The optimum solution is to send the initial pulse to produce a flip angle $a<90^{\circ}$ and perform the measurement while the nuclei are dephasing. Thus, the normal process of spins dephasing is boosted with a gradient pulse (signal decrease) 
and then, the gradient is reversed and nuclei start to rephase (signal increase) generating an echo. A signal can easily be detected from this GRE process with significantly reduced time in comparison to SE sequences. This set of two gradients (dephase, rephase) of equal amplitude and duration but of opposite sign is commonly expressed as bipolar gradient.

A time diagram of the GRE sequence can be appreciated in figure 1.12.

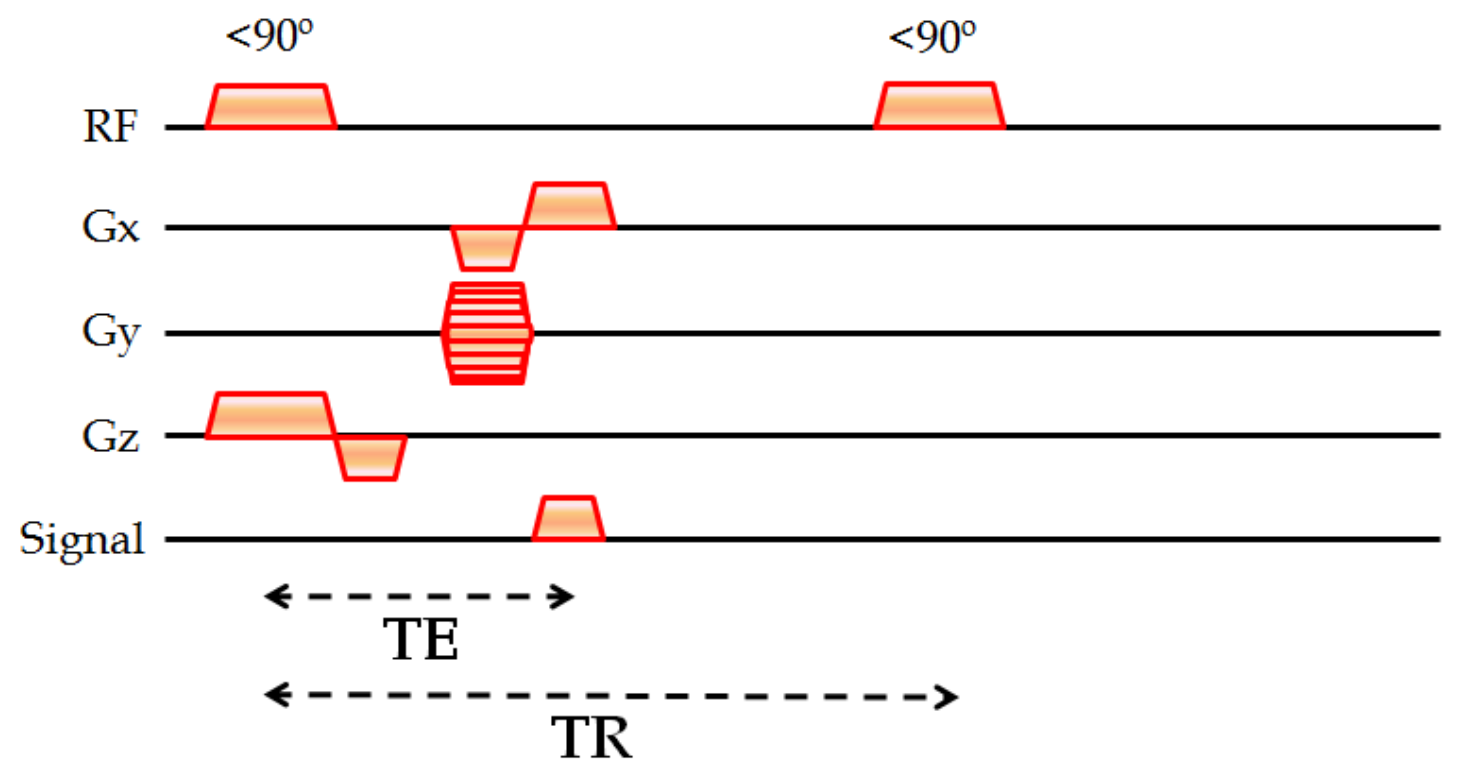

Figure 1.12. Gradient-echo sequence pulse diagram

As it can be observed in the pulse sequence diagrams, the slice selection gradient $(G z)$ is activated simultaneously during excitation by the RF pulse. It can also be noted that the echo acquisition is performed during the activation of the frequency-encoding gradient $(G x)$ and that the phase-encoding gradient (Gy) amplitude is modified in each TR cycle.

The GRE sequences have permitted the acquisition of an entire volume of data (3D) in a reasonable time due to the speed of the gradients and the reduced flip angle at excitation. For applications that require a high spatial resolution in the three directions of space, GRE sequences can be implemented in a 3D acquisition mode employing two phase encoding gradients, thus, no slice selection is performed and resultant images are usually named as partitions. 


\subsubsection{MR in the study of bone}

The use of MR for the study of bone has been traditionally limited due to the low intensity of bone tissue at MR images. This reduced signal intensity is due to the fast signal decay produced by the typical short T2 $(250-500 \mu \mathrm{s})$ of water situated in bone micropores next to hydroxyapatite crystals [4].

Nevertheless, in recent years, a growing number of studies have been performed that applied MRI to the study of bone. Two MRI techniques may be used to characterize bone [11]:

- Mineralized bone can be assessed with MRI using T2* decay characteristics that measure field inhomogeneities caused by susceptibility differences at the marrow-bone boundaries. The susceptibility gap between bone and fatty marrow is approximately 3 parts per million (ppm) and thus affects the local field distribution at the bone-marrow interface. This technique provides information about bone density as well as bone structure.

- Using high-resolution MRI, the trabecular bone structure may be quantified directly by the post-processing of medical images with computer-based analysis software, as it is developed in the present thesis. This technique requires high-field scanners with fast gradients and surface coils permitting the acquisition with high SNR conditions.

Modern high-field clinical scanners with fast gradients and optimized coil design can provide very high spatial resolutions in the order of $100-200 \mu \mathrm{m}$ in the three directions of space while maintaining a satisfactory SNR. Several studies at high spatial resolution have been developed in vivo in the phalanges [38], distal radius [39], femur [40], vertebrae [39] and calcaneus [39].

Currently, the two primary techniques used for high spatial resolution MRI of trabecular bone are variants of the basic GRE and SE pulse sequences. A GRE pulse configuration is used with the application of residual magnetization 
destruction or spoiling during relaxation also called spoiled GRE [41]. In another way, fast large-angle spin-echo (FLASE) pulse configurations, mainly based on SE sequences have been applied to the study of trabecular bone structure [31]. Steady-state free precession (SSFP) has recently proven its potential in various applications because of its short scan time and high SNR efficiency [42]. SSFP is essentially a gradient-echo sequence in which TR is chosen to be as short as technically feasible and imaging gradients in all directions are fully rewound at the end of each repetition period. SSFP is known to be highly sensitive to off-resonance effects resulting from field inhomogeneity and chemical shift dispersion [43]. Some solutions like the use of constructive interference techniques in steady-state (CISS) have been proposed in order to minimize the banding artifacts produced by susceptibility differences.

Fatty marrow (which prevails in the distal extremities) is composed of fatty acid triglycerides that possess multiple chemically-shifted resonances. The fundamental difference between the two families of sequences initially suggests that SE-based pulse sequences are more appropriate for imaging regions that are prone to susceptibility-induced local fields, such as in trabecular bone marrow. Such chemical shift effects or local field differences are manifested as dark-bright bands in the resulting MR images, especially in GRE sequences, which have a higher sensitivity to susceptibility differences. Although the phase dispersion is eliminated in the spin echo, the signal decays as $\exp (-T E / T 2)$. The use of a phase reversal pulse may require a significantly longer time to form an echo compared to a gradient echo-a consideration that would favor gradientecho-based pulse sequences.

Both classes of imaging sequences are used: while Majumdar et al. used 3D GRE sequences [39, 42], Wehrli et al. employed SE sequences for the trabecular bone characterization [30,40]. Differences in susceptibility effects cause the apparent size of the trabeculae to vary and measures obtained with the individual sequences may not be compared. Despite their short acquisition time, there exists a slight amplification of the trabecular dimensions which is 
more pronounced in the GE images. This effect is due to the previously mentioned chemical shift artifact and can be minimized using a reduced TE and increasing the receiver bandwidth. However, the bandwidth increment may penalize the SNR. Optimized GRE sequences in terms of spatial resolution, SNR and chemical shift can provide an efficient depiction of the bone-marrow interface and $\mathrm{SE}$ sequences with relatively long acquisition times can be avoided [44].

\subsubsection{Acquisition requirements for trabecular bone characterization}

In order to perform efficient characterizations of the trabecular bone properties from in vivo MR images, a very high spatial resolution is required in acquisition. A very satisfactory spatial resolution with good SNR can be obtained in the three directions of space using a 3 T MR system, whereas slice thickness needs to be significantly increased in $1.5 \mathrm{~T}$ in order to maintain the SNR. However, several imaging problems arise, which are inherent to the use of high spatial resolution MRI and special technical parameters have to be always considered. As previously mentioned, the main size of bone trabeculae is around 80 to 150 $\mu \mathrm{m}$. These reduced dimensions determine the spatial resolution required in acquisition as practically the most critical MR parameter. Voxel size cannot be directly reduced to the order of the structure size in order to be able to extract structural information due to SNR limitations. In order to quantify such SNR penalties, if all other parameters are maintained, a diminution of the spatial resolution from $1 \mathrm{~mm}^{3}$ to $150 \mu \mathrm{m}^{3}$ in the voxel size supposes a 300 -fold SNR reduction.

One of the reduced possibilities to boost SNR is to reduce the size of the receiving RF coil. The SNR for a circular surface coil scales inversely with coil radius. Smaller and dedicated coils may be used for each specific purpose with aim of optimizing the SNR in acquisition for cancellous bone characterization.

The much shorter relaxation time of protons in marrow lipids significantly improves the SNR of the images, specially at distal extremities, where a 
considerable amount of fatty bone marrow exists. However, the local resonance differences between bone and fatty bone marrow produce the chemical shift artifact that must be minimized by reducing the TE and increasing the receiver bandwidth. Flow artifacts produced by the blood circulation through the vessels in the volume of acquisition are reduced also with the decrease in the TE.

\subsection{Image processing and quantification of trabecular bone}

High resolution MRI can be used for the image processing and quantification of different parameters that may act as bone disease biomarkers. Microarchitecture deterioration induced by osteoporosis supposes an increase in the fracture risk due to trabeculae disorganization. Many different approaches exist for the quantification of trabecular bone microstructural properties. These image-based measurements applied to MR trabecular bone images may act as a kind of virtual bone biopsy that permits a complete characterization of cancellous bone tissue properties in a completely noninvasive way.

\subsubsection{Image processing in digital radiology. Applications to the study of trabecular bone.}

In recent years, the evolution of computing capabilities and the development of new medical imaging techniques based on digital acquisitions, such as CT or MRI, have allowed the improvement in clinical and radiological examinations assessed by engineering sciences and large scale computation. The standardization of digital image formats for medicine through common consensus, like the Digital Imaging and Communication in Medicine (DICOM), which is extensively used nowadays, have eased the sharing of clinical cases and studies among different centers. Furthermore, the applicability of digital image processing techniques to extract quantitative information from medical 
images which, at priori cannot be detected or quantified visually by the clinician are changing the current radiological workflow.

Acquisitions from CT or MRI of consecutive cuts in certain regions of the human body have facilitated the development of 3D volumetric reconstructions of different structures with a very high reliability. The application of engineering techniques historically used in civil, aerospace or electronics is growing in the biomedical area contributing to the development and advancement of biomedical engineering. Even more, these image-based disciplines applied to the study of living organs and tissues in our body has supposed a revolution of the healthcare process.

The bone tissue can also benefit from the application of image computing algorithms to medical imaging acquisitions. In the present thesis, different image processing methods to analyze trabecular bone structure from MR images are presented. Several texture characteristics regarding morphology, connectivity, complexity and anisotropy can be extracted. Also, structural engineering techniques like the FE method can be applied in order to analyze the mechanical response of individualized trabecular bone reconstructions obtained from in vivo MR images. These approaches are highly useful for the research and advance in new imaging biomarkers of bone diseases like osteoporosis.

\subsubsection{State of the art in trabecular bone characterization. Quantification of trabecular bone from MR images. Basis and aims for this dissertation.}

The cancellous bone structure was traditionally analyzed by means of stereology, studying its histomorphometry from sections [45], a technique where the third dimension is estimated by inference. This practice lacks of accuracy and certainty since trabecular bone architecture is anisotropic and inherently 3D [46]. This issue was recognized early and it was developed a better method based on serial sectioning techniques from which 3D images could be reconstructed [7]. In spite of the improvements, these techniques were 
rapidly superseded by the application of nondestructive imaging techniques like $\mu \mathrm{CT}[6,47,48]$. X-ray-based acquisition modalities like CT or $\mu \mathrm{CT}$ present a high sensitivity to bone tissue, due to the high radiation absorption observed in calcified structures, in comparison with the soft tissues.

In vivo, however, some difficulties regarding radiation dose arise and limit the achievable resolutions even at peripheral anatomic sites. A high performance High Resolution peripheral Quantitative Computed Tomography (HR-pQCT) system was built in the Institute for Biomedical Engineering at the Swiss Federal Institute of Technology in Zurich [49], where images at a voxel size of $165 \times 165 \times 165 \mu \mathrm{m}$ could be obtained [50]. More recently, a commercial HRpQCT scanner has become available (Xtreme $^{\mathrm{TM}}$, Scanco Inc., Brüttisellen, Switzerland), designed for imaging the distal radius. The system uses conebeam scanning and reconstruction at a nominal voxel size of 80 to $90 \mu \mathrm{m}^{3}$ [50]. The prospect of performing structure analysis in the axial skeleton by clinical MDCT has recently also been demonstrated. A very interesting study analyzed trabecular bone images in the vertebrae at $250 \times 250 \times 500 \mu \mathrm{m}^{3}$ voxel size, showing structural parameters to distinguish fracture from nonfracture subjects [51]. However, the radiation dose at which the studies were performed was as high as $77 \mathrm{mGy}$, which is more than twice that of the reference level for body CT. By contrast, the effective dose for HR-pQCT for a 2.8 minute scan has been quoted as $0.003 \mathrm{mGy}[50]$, which is almost negligible.

The high innate contrast between bone (which appears with background intensity) and bone marrow, along with its noninvasiveness, render MRI uniquely suited for imaging trabecular bone microarchitecture in vivo. High resolution MRI can be used in the postprocessing and quantification of different parameters that may act as disease biomarkers. High spatial resolutions in a reasonable acquisition time can be achieved with the increased signal-to-noise ratio of the 3 T MR scanners. Regarding bone evaluation, these images can give information of the cancellous structure with great details.

It is useful to distinguish between methods that attempt to resolve trabecular bone microarchitecture directly vs. those that seek to obtain structural 
information indirectly without the need to resolve individual trabeculae. Due to the capability of obtaining a high signal-to-noise ratio (SNR) with the use of dedicated surface coils, high-resolution MRI is generally performed at peripheral skeletal locations only such as the distal tibia [52], calcaneus [53, 54], and wrist [55-57]. The feasibility of assessing texture in the proximal femur, albeit at considerably lower resolution, has also been explored [58].

Other approaches have been investigated as well, such as measurement of the trabecular bone volume fraction, exploiting the reduction in signal amplitude due to fractional occupancy of the voxel by bone [59]. Finally, indirect detection of trabecular bone microstructure by exploiting amplitude modulation from protons signals caused by the structural regularity of the trabecular bone network, has also been investigated [60].

In the present thesis, a complete assessment of morphometry and mechanical properties of the cancellous bone was performed to in vivo high spatial resolution MR images.

As trabecular thinning and pore size increment are clear biomarkers of osteoporosis [3], it seems quite relevant to develop a structural characterization of trabecular bone based on its morphological parameters. The most relevant morphological values in the characterization of trabecular bone are the bone volume to total volume ratio (BV/TV), mean trabecular thickness (Tb.Th), mean trabecular separation (Tb.Sp) and trabecular number $(T b . N)$ [4, 54, 55]. The methods developed to obtain such morphological indicators are treated in the present manuscript.

Trabecular shape and surfaces complexity appear to be also altered under pathological conditions, with less irregular and less complex bone surfaces [61]. Using the fractal theory, it is possible to quantify structures with complex form through the fractal dimension $(D)$, a parameter which indicates how an irregular structure tends to fill space after the observation at different scales [62]. Although there are a large number of definitions of $D$ with different computation methods, the box-counting dimension which is also known as Minkowski-Bouligand dimension, Kolmogorov capacity or Kolmogorov 
dimension has been widely extended in medical image processing due to the easiness of implementation [63].

The trabecular bone relationship of $D$ with other bone quality parameters has been assessed in different studies [55, 61, 64]. However, only a reduced number of studies have analyzed the 3D complexity of the trabecular bone network of volumetric reconstructions obtained from multidetector row CT (MDCT) and synchrotron microtomography $(\mu \mathrm{CT})$ images [50,65]. In MRI, the use of 3D algorithms for the quantification of trabecular bone properties has been limited at $1.5 \mathrm{~T}$ scanners by the reduced achievable spatial resolutions. However, at higher field strengths, it is possible to improve the in vivo 3D characterization of the trabecular structure since isotropic voxels with higher spatial resolutions can be obtained without compromising the signal-to-noise ratio (SNR) [66]. In the present thesis, algorithms for the analysis of the 2D fractal dimension $\left(D^{2 D}\right)$ of the trabecular bone have been implemented. An extension to a generalized $3 \mathrm{D}$ version of the algorithms has been also developed in order to improve the volumetric fractal characterization of the structures by the calculation of the 3D fractal dimension $\left(D^{3 D}\right)$.

The homogeneous thinning of a trabecular structure, while affecting parameters of scale, does not entail a change in the structure's topology as long as no connections are broken or a trabecular plate is perforated. In the case of postmenopausal osteoporosis the deterioration mechanism involves gradual deepening of osteoclastic resorption pits that eventually lead to fenestration of plates and disconnection of rods. Thus bone loss is often compounded by disproportionately large topological changes [67, 68]. Feldkamp et al. showed that cancellous bone network connectivity can be expressed in terms of its topological characteristics [6].

A method designed for unambiguous determination of the topological classes of digitized structures is digital topological analysis (DTA) [69]. This kind of analysis, which has more recently been applied to digital images of trabecular bone, relies on the three main local topological entities (number of objects, tunnels, and cavities), except that these relationships are examined for each 
bone voxel in its neighborhood [70]. The topological information quantified in a voxel-by-voxel basis can be summarized in parameters giving information about structure topology like the total erosion index (TEI) and the surface-tocurve ratio $(S C R)$. For the assessment of topology, a 3D skeletonization algorithm and the DTA method have been implemented for the estimation of TEI and SCR from in vivo MR images.

The structural anisotropy, most manifest in the preferred orientation of the trabeculae, is a direct consequence of Wolff's law that conveys that bone models and remodels in response to the stresses to which it is subjected [71].

Structural orientation has received a great deal of attention as an indirect predictor of the bone's mechanical properties. The well known mean intercept length (MIL) method for trabecular bone anisotropy analysis was implemented in its 2D and 3D versions. Furthermore, a more recent method, called tensor scale developed by Saha et al. in 2D may be applicable to in vivo imaging of trabecular bone structure in order to provide information on trabecular bone orientation. Bone trabeculae seem to preferentially orientate along the major stress lines and the preferential loss of transverse trabeculae appears to be a hallmark of osteoporosis [72]. In the present thesis, the tensor scale method is implemented in 2D and a new and original $3 \mathrm{D}$ version of the tensor scale algorithm is presented as a tool for structural anisotropy analysis of high spatial resolution 3D reconstructions of trabecular bone.

Finally, any approach to the quantification of trabecular bone microstructure mechanical properties should be also considered in order to evaluate the fracture risk.

Fractures are the consequence of mechanical overloading. The evaluation of elasticity and fluency limits of different materials and structures is commonly assessed by mathematics and engineering techniques. It seems reasonable to consider that bone fracture risk should also be analyzed by exact and precise engineering methods. Nevertheless, although bone mechanical properties can be directly extracted from ex vivo experiments, the mechanical analysis of bone in vivo has always been limited to indirect measurements since there is no 
physical interaction with the sample. Therefore, the investigation in new methods to characterize trabecular bone microarchitecture alterations produced by the disease is crucial.

Structural mechanical simulations are currently being developed in the engineering field by using the FE method, which consists in the division of a continuum into discrete elements that define a linear system of equations with displacements and forces to be solved. A study of the mechanical properties of the trabecular bone can be obtained by the application of the FE method to the trabecular bone structures [73]. However, since such architectures present changes at microscopic scale, elements with significantly reduced dimensions are required for the application of the FE method, which in these conditions is also called as microfinite element, micro-FE or $\mu \mathrm{FE}$ method.

Trabecular bone $\mu \mathrm{FE}$ models can be simulated under different mechanical conditions in order to obtain stresses, strains and displacements of the corresponding architecture. Furthermore, elasticity characteristics can be calculated from the simulations and indicators such as the apparent Young's modulus can be obtained.

In the present thesis, a fast and original algorithm for the creation of $\mu \mathrm{FE}$ meshes from binarized 3D trabecular bone reconstructions has been implemented. Also, an automated routine for model generation and interaction with the FE solver has been developed.

As it has been introduced, trabecular bone tissue properties can be analyzed from a variety of parameters that can be extracted in vivo from high spatial resolution MR acquisitions. Such advances may not only be useful for a better diagnosis of the disease, but also to evaluate the efficacy of the existing therapies. 


\section{Methodology}

In the present chapter, all the processes related to image acquisition and protocols are initially presented. Thereafter, image processing and analysis techniques and the development of the different algorithms used, morphometry analysis techniques and mechanical simulations are widely explained. Also, the processes followed for the validation of the methodology using animal models and $\mu \mathrm{CT}$ acquisitions are assessed. Finally, the statistical analysis methods employed in the present thesis in order to evaluate the applicability of the developments and algorithms are commented.

\subsection{MR images acquisition}

\subsubsection{Region of acquisition}

The anatomical area or region selected for MR images acquisition and later the cancellous bone analysis was located in the wrist. To be concrete, the acquisition stack was planned at the level of the distal radius metaphysis. This region was chosen because of its high incidence of osteoporotic fractures and also due to the relatively high and homogeneous SNR that can be obtained using MRI because of its superficial location. Furthermore, long bone metaphyses usually contain high amounts of trabecular bone. Since trabecular bone analysis and quantified parameters must not be influenced by subject dominancy, the acquisitions were performed on the non-dominant wrist for each patient (right in left-handers and vice versa).

\subsubsection{MR hardware}

The in vivo acquisitions for the analysis of trabecular bone microarchitecture were performed in a $3 \mathrm{~T}$ Achieva (Philips Healthcare, Best, The Netherlands) 
magnet at Quirón Valencia Hospital (see Figure 2.1). Due to the increased difference between parallel and anti-parallel spin populations, the use of a $3 \mathrm{~T}$ magnetic field for spin magnetization permitted the acquisition with a significantly higher SNR in comparison to a $1.5 \mathrm{~T}$ scanner (almost two-fold under ideal conditions [34]). Furthermore, spatial resolutions could be considerably improved simultaneously in the three directions of space.

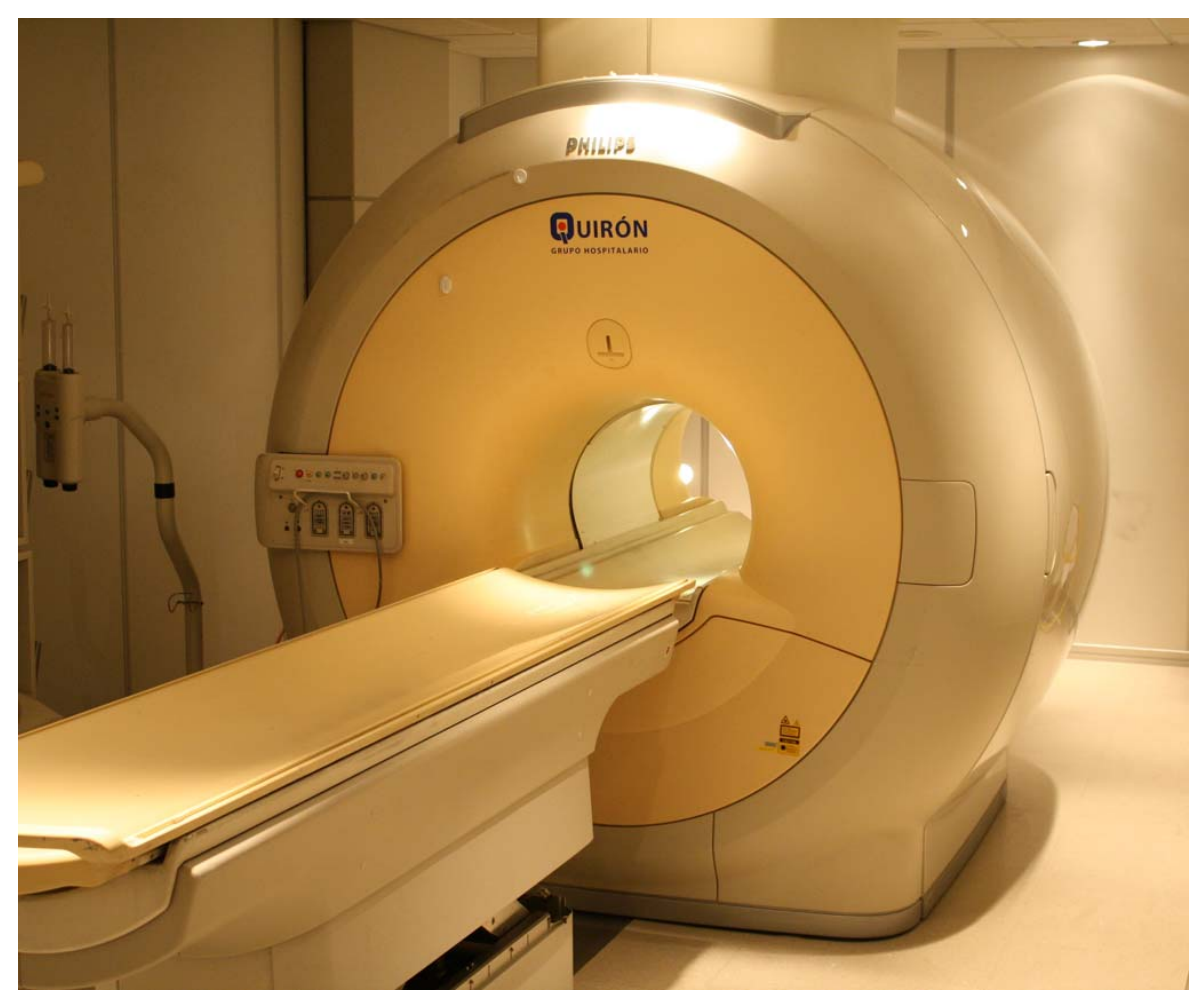

Figure 2.1. 3T MR system at Quirón Valencia Hospital manufactured by Philips Healthcare, Best, The Netherlands.

The gradient system consisted of a Dual Quasar, with a slew-rate of 200 $\mathrm{mT} / \mathrm{m} / \mathrm{ms}$ and a maximal gradient strength of $80 \mathrm{mT} / \mathrm{m}$. A four-channel phased-array surface coil especially designed for the wrist region was used exclusively for signal reception (see Figure 2.2). Dedicated wrist fixators adapted to the coil shape were used to immobilize patient's wrist and avoid movement artifacts in the images. 

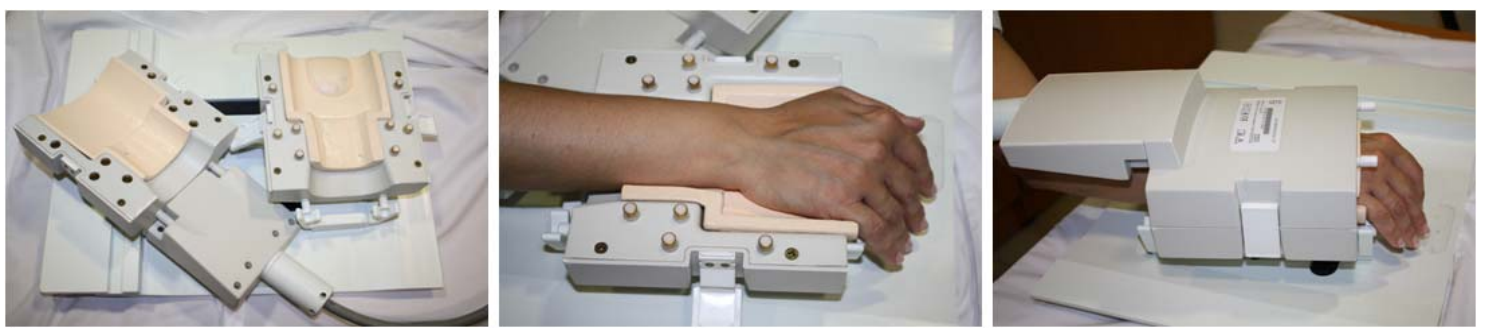

Figure 2.2. Wrist phased array surface coil with four channels. An optimum adaptation of the coil to the wrist region was achieved with an important SNR increment.

\subsubsection{MR pulse sequences}

The acquisition mode was decided to be 3D since a very high spatial resolution was required in the three directions of space and the SNR needed to be maximized. The designed pulse sequence for image acquisition consisted of a spoiled T1-weighted gradient echo, where a spoiler gradient is used to destroy any remaining transverse magnetization after the readout gradient. A heavy T1 weighting was needed in order to enhance bone marrow signal, thus, a short echo time (TE) of $5 \mathrm{~ms}$ and repetition time (TR) of $16 \mathrm{~ms}$ in combination with a flip angle $(\alpha)$ of $25^{\circ}$ were used to maximize contrast. A receiver bandwidth of 167.6 Hz/pixel was chosen for a satisfactory SNR. The acquisition volume was divided into 60 axial partitions, each one with an in-plane matrix size of $512 \mathrm{x}$ 512 pixels. Partition thickness and pixel size were set to achieve isotropic voxels of $180 \mu \mathrm{m}$ per edge. To author knowledge, this was the first reported experiment with a high and isotropic spatial resolution for trabecular bone analysis in vivo [66]. In order to boost the signal and minimize noise, the number of averaged acquisitions was set to 3 . With aim of optimizing the acquisition time and easing the viability of the acquisition in the clinical routine, the sensitivity encoding (SENSE ${ }^{\mathrm{TM}}$ ) parallel imaging technique was used in the in-plane phase direction with an acceleration factor of 2 [74]. Parallel imaging techniques have been shown to be feasible in high-spatial resolution MRI acquisitions of the trabecular bone [75]. Total acquisition time was 5 minutes and 42 seconds. 
An overview of the designed MR sequence details can be appreciated in Table 2.1:

\begin{tabular}{|c|c|}
\hline MR Parameter & Value \\
\hline Acquisition mode & $3 \mathrm{D}$ \\
\hline Pulse sequence & Gradient echo, spoiled (T1) \\
\hline Number of partitions & 60 \\
\hline Partition orientation & Axial \\
\hline Field of view & $90 \mathrm{~mm} \times 90 \mathrm{~mm}$ \\
\hline Matrix size & $512 \times 512$ \\
\hline Voxel dimensions & $180 \times 180 \times 180 \mu \mathrm{m}^{3}$ \\
\hline Slice gap & 0 \\
\hline $\begin{array}{c}\text { Parallel imaging factor } \\
\text { (in-plane phase direction) }\end{array}$ & 2 \\
\hline $\begin{array}{c}\text { Parallel imaging factor } \\
\text { (through-plane phase direction) }\end{array}$ & 0 \\
\hline Bandwidth & $167.6 \mathrm{~Hz} /$ pixel \\
\hline a & $25^{\circ}$ \\
\hline TE & $5 \mathrm{~ms}$ \\
\hline TR & $16 \mathrm{~ms}$ \\
\hline Number of averages & 3 \\
\hline Acquisition time & 5 min. $42 \mathrm{~s}$ \\
\hline
\end{tabular}

Table 2.1. Details of the sequence design implemented for MR images acquisition. A high spatial resolution is obtained in a reduced acquisition time.

In figure 2.3, an example of a wrist image acquired with the specified MR pulse sequence can be appreciated.

As shown, a satisfactory detail depiction is achieved with the proposed MR sequence in a reasonable acquisition time. However, although images present a good global quality, some acquisition related phenomena must be corrected and images must be initially processed before being used for the extraction of properties or the quantification of possible disease indicators. 


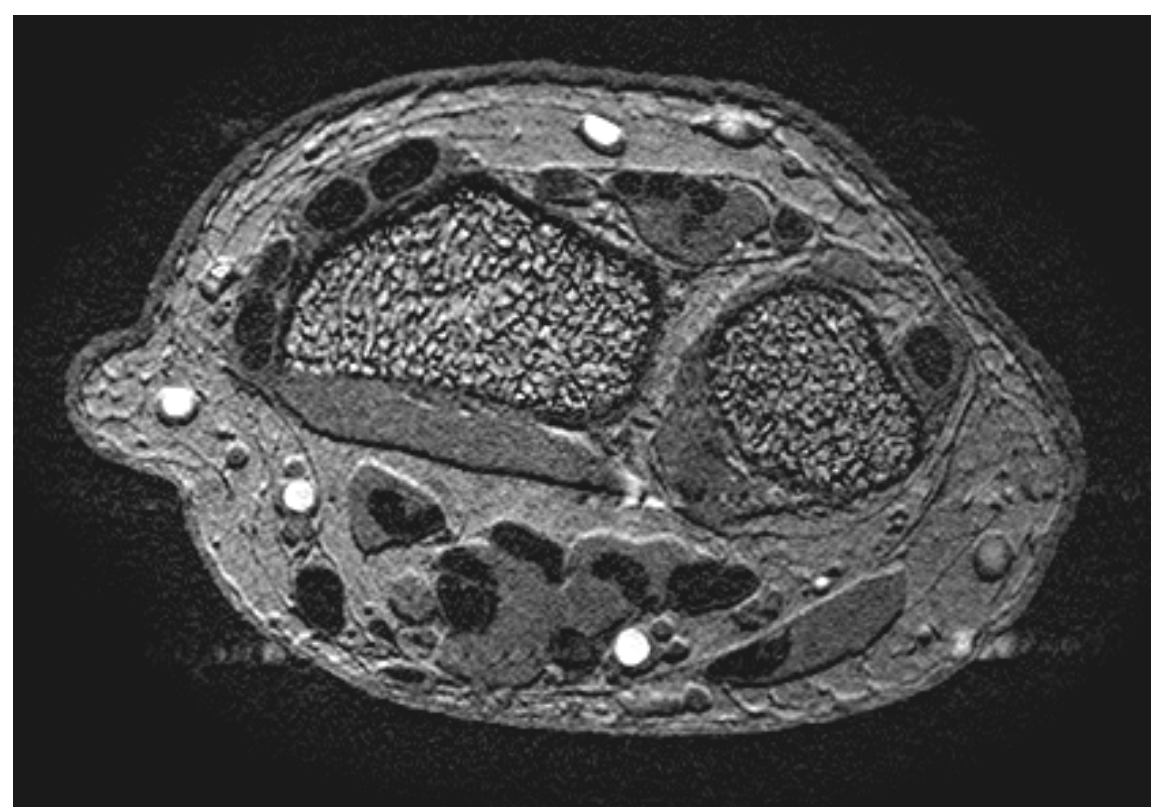

Figure 2.3. Axial acquisition performed in the radius metaphysis using the defined 3D-GE spoiled sequence. Note the efficient trabeculae depiction in dark, and brighter bone marrow signal surrounding the cancellous bone.

\subsection{Image processing}

The image processing steps required for morphometric and mechanical properties extraction of the trabecular bone acquisitions are detailed in this section. The reference frame used for all the developments is: z-axis corresponding to the longitudinal direction of the radius; $x$-axis corresponding to the anterior-posterior direction and y-axis corresponding to the left-right direction. Although the $3 \mathrm{D}$ version of the pre-processing algorithms is explained in the manuscript, the initial developments in the thesis were made in $2 \mathrm{D}$ and have been migrated to $3 \mathrm{D}$ in the last stage of our work. It is considered that the understanding of their $2 \mathrm{D}$ versions is straightforward. An initial segmentation process is performed in order to isolate the trabecular bone volume. Once the trabecular bone region is isolated, small signal intensity heterogeneities exacerbated with the use of surface coils are statistically corrected by determining the local threshold intensities. Also, as the true 
trabecula dimension is smaller than the acquired pixel size, partial volume effects are minimized by interpolation. Finally, a complete discrimination between bone and marrow voxels was achieved after binarization. All processing algorithms were implemented in Matlab R2007a (Mathworks, Natick, MA, USA).

\subsubsection{Segmentation}

The images acquisition for the characterization of the trabecular bone structure of radius was undertaken in a series of axial slices of the wrist and therefore covering a region greater than the volume that is actually relevant for the cancellous bone analysis. The 3D image reconstruction and parameters calculation were initially preceded by a delineation of regions in order to isolate trabecular bone tissue and extract its properties.

Two different segmentation methods were implemented. First, an automated segmentation algorithm was developed in order to extract the regions corresponding to radial trabecular bone from each slice [76]. The segmentation contour was automatically adapted to the internal cortical shape of the radius after the definition of a starting point in the middle of the trabecular bone region. Also, a semi-automated segmentation method was implemented, which consisted in the delimitation of a parallelogram of trabecular bone. This volumetric segmentation is of special interest for mechanical simulations of trabecular bone, where samples of material with parallel faces are required for proper analysis.

\section{$\underline{\text { Automated segmentation }}$}

The solution to the detection of trabecular bone contour by the delineation of internal cortical bone shape consisted in the detection of areas in which a great 
grayscale intensity variation was observed between the same pixels in adjacent slices.

A base image with a clear border definition between spongy and cortical bone was used to initialize the segmentation algorithm. Intensity variations between adjacent slices were detected and a contour can be depicted. This contour can be adjusted to the desired cortical contour through the application of Snakes [77]. The last boundary fitting is taken as an initial contour for the next slice, then, new deformable models are applied again and a satisfactory fit is obtained. The last fitted contour is taken as the initial contour for the next image. An iterative process through the rest of slices permits the segmentation of the entire series of images automatically.

The initial contour estimation is the first step in the algorithm and consists in a combination of range averaging filters with a final binarization.

\section{Estimation of the initial contour}

The first step for the estimation of the initial contour is the application of a 3D rank filter to the images. This filter consists in taking a neighborhood of $N \times N \times M$ for each pixel of the images, and assigning the maximum difference of intensities in the neighborhood to the corresponding pixel value. The expected result after the application of this filter is a series of images in which pixel intensities for neighborhoods with maintained low values can be differentiated from the high values of pixels corresponding to neighborhoods where there are extreme values of intensity. Therefore, to discriminate trabecular and cortical bone, there should be intensity variations in the cancellous bone neighborhoods and no abrupt changes expected in the cortical bone intensities. An example of the output of the 3D rank filter can be appreciated in figure 2.4. 

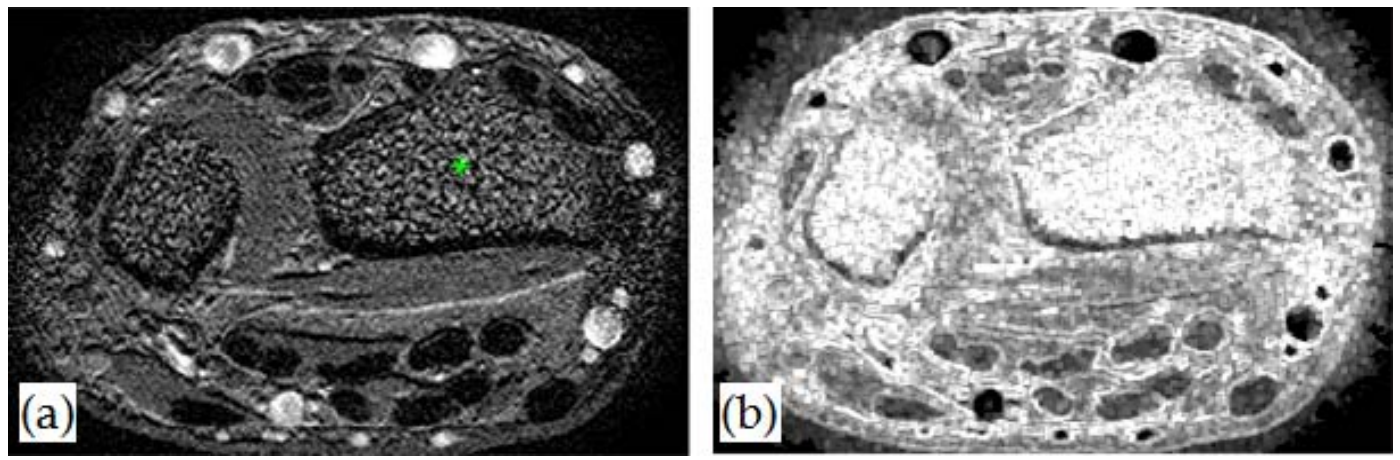

Figure 2.4. In (a), image used for initialization of the segmentation algorithm. The green dot indicates the area where a mouse click has been done in order to start the automatic segmentation process. In (b), image (a) after applying a 3D rank filter with a $3 \times 3 \times 3$ neighborhood. The trabecular bone region shows higher intensities than those of cortical bone.

If a 3D circular averaging filter (pillbox) is applied to the resultant images of the 3D rank filter, the results can be significantly improved, as it is observed in figure 2.5.

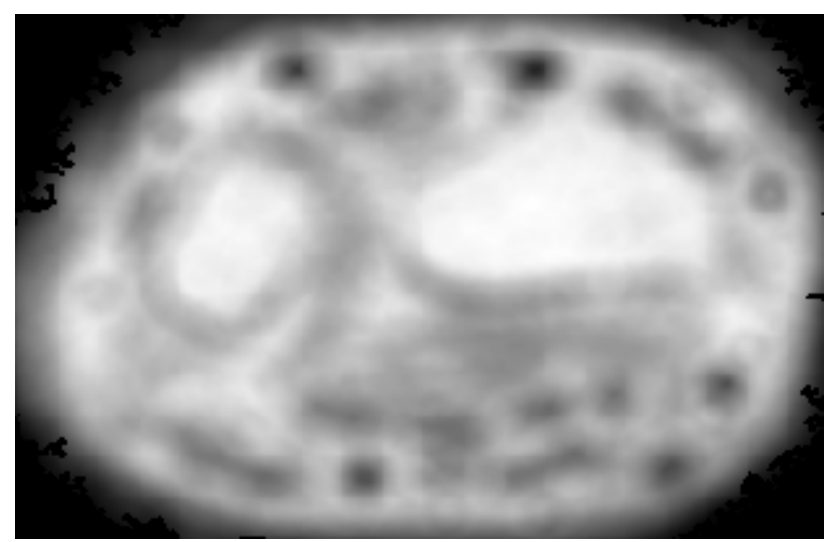

Figure 2.5. Result of the application of a pillbox 3D filter to figure 2.4 (b).

For a more detailed contour delineation, a two-dimensional (2D) range filtering is performed again with a $3 \times 3$ mask. Now, changes in intensity occur in the boundaries between cortical and trabecular bone and images with enhanced contour delineation are obtained, as it can be seen in figure 2.6a. However, contours uniformity can be still improved by the application of an average filter with a $3 \times 3 \times 3$ mask, as it may be noted in figure $2.6 \mathrm{~b}$. 

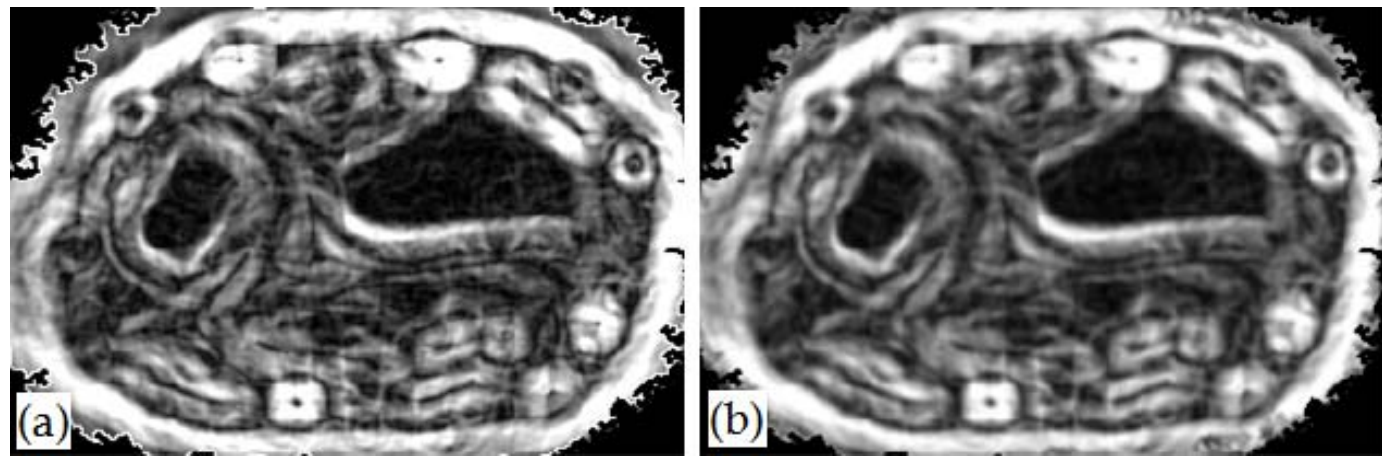

Figure 2.6. In (a), results after the application of a $3 \times 3$ rank filter. Contours are depicted with a high sensitivity. In (b), a better contours uniformity is obtained after the application of an average filter with a $3 \times 3 \times 3$ mask.

Finally, resulting images are binarized and regions with significantly small areas are removed (figure 2.7a). A distance transform algorithm is applied then and considering the initial introduced point, the initial contour is calculated, as it can be appreciated in figure $2.7 \mathrm{~b}$.

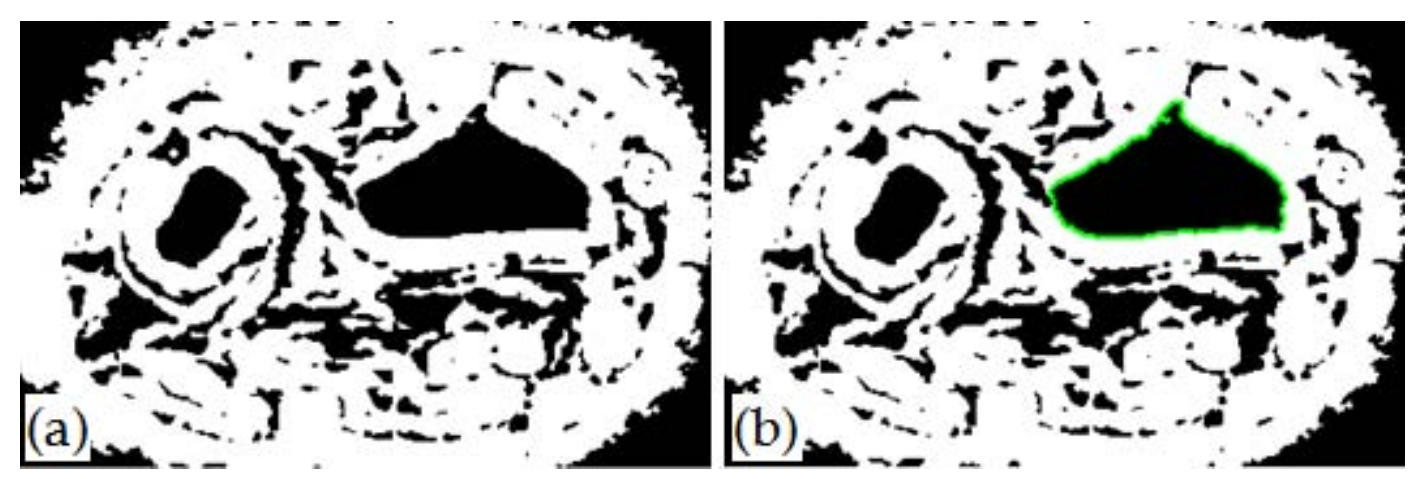

Figure 2.7. In (a), binarized image after the application of a threshold to the images resulting from the filter shown in figure 2.6b. In (b), initial contour detection by the calculation of the distance transform from the initial point introduced interactively for the automated segmentation.

\section{Final adjustment}

The initial contour calculated is used as a starting condition for the gradient vector flow (GVF) snake algorithm in order to achieve the definitive fitting of the contour to the cortical bone shape (see figure 2.8). Once the contour is finally adjusted for the first slice, the calculated contour is used as an initial condition for the adjacent slice snakes segmentation algorithm. 
The parameters to be set for the GVF field calculation are two: $\mu$, which is a regularization coefficient whose value increases with the image noise, and the number of iterations. In this case $\mu$ was adjusted 0.2 and the number of iterations to 100 .

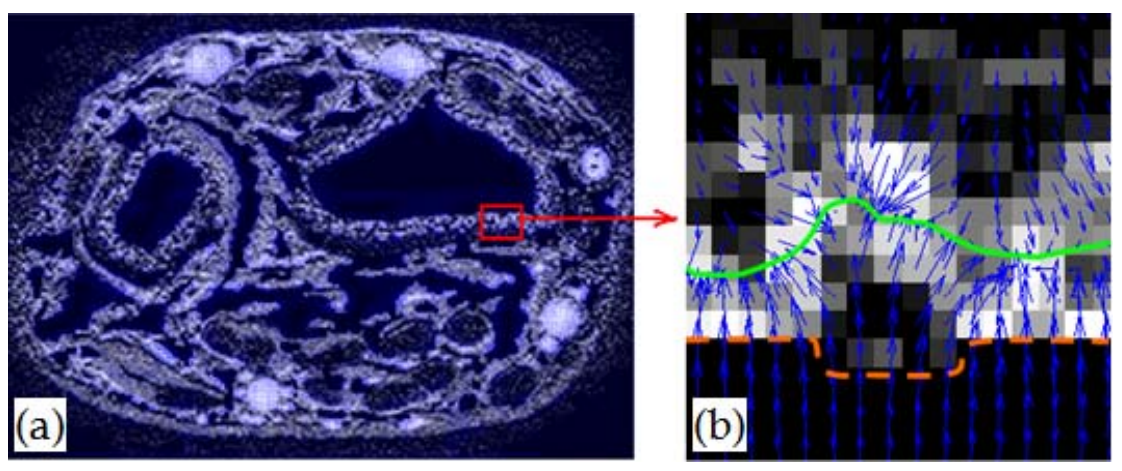

Figure 2.8. In (a), calculation of the GVF map to the image resultant from the multiplication of the anatomical slice by the initial calculated contour. In (b), zoomed region showing the initial contour estimated in the first step of the segmentation (orange) and the final contour (green) after the application of GVF vectors (blue).

After the calculation of the segmentation contours for each slice, a series of contours defining the segmentation of the trabecular bone of the radius are obtained. An example of series segmentation can be observed in figure 2.9. 


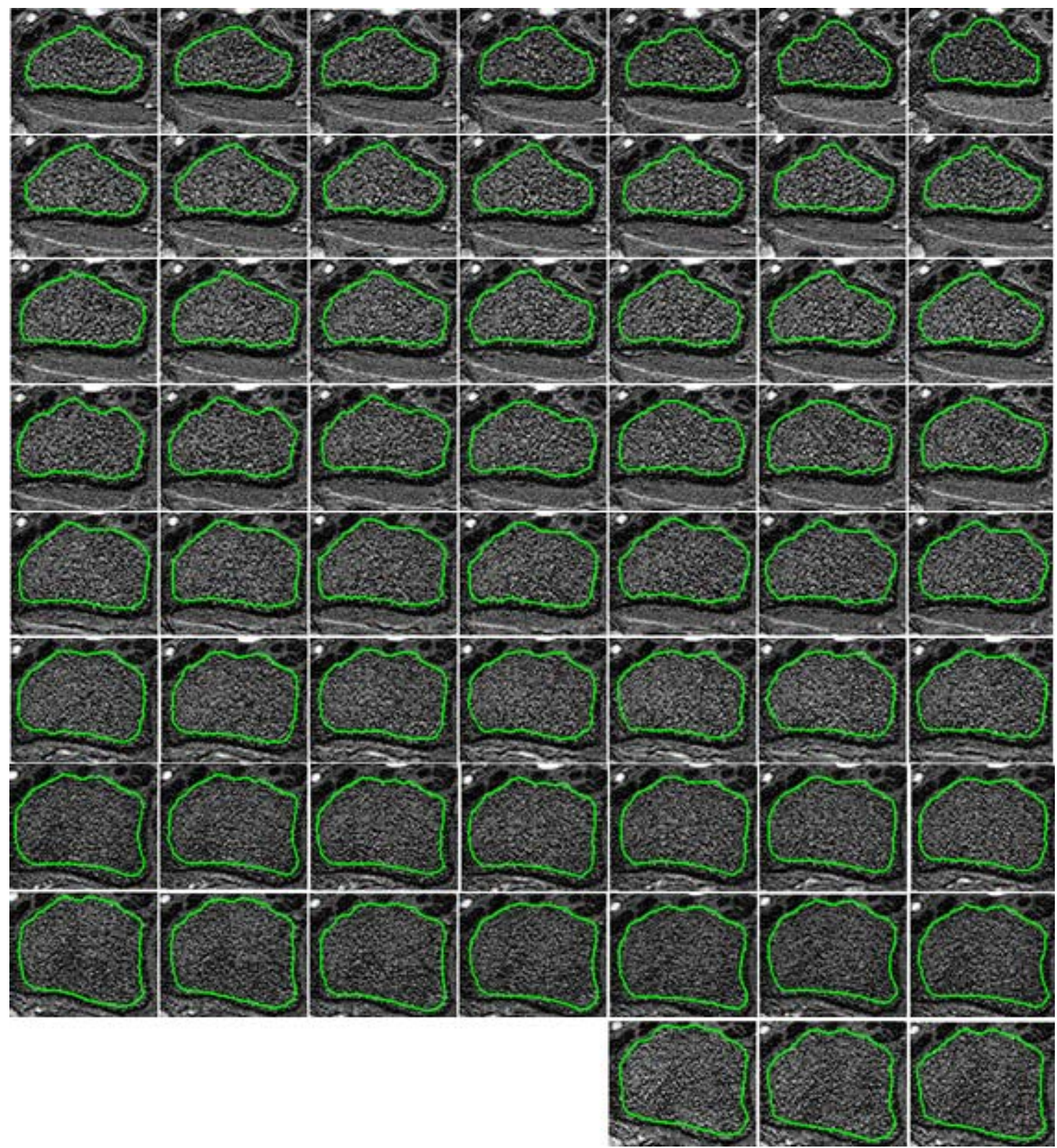

Figure 2.9. Results of the segmentation of the trabecular bone region in the radius distal metaphysis. The images are ordered from left to right and top to down. In green, the final contours of the segmentation.

\section{$\underline{\text { Semi-automated segmentation }}$}

The semi-automated segmentation of the trabecular bone from MR images was simply performed by placing a rectangular ROI in the first slice corresponding to the most proximal position and thereafter propagated to the rest of slices. Segmented areas were verified to exclusively contain marrow and trabecular bone (see figure 2.10). 


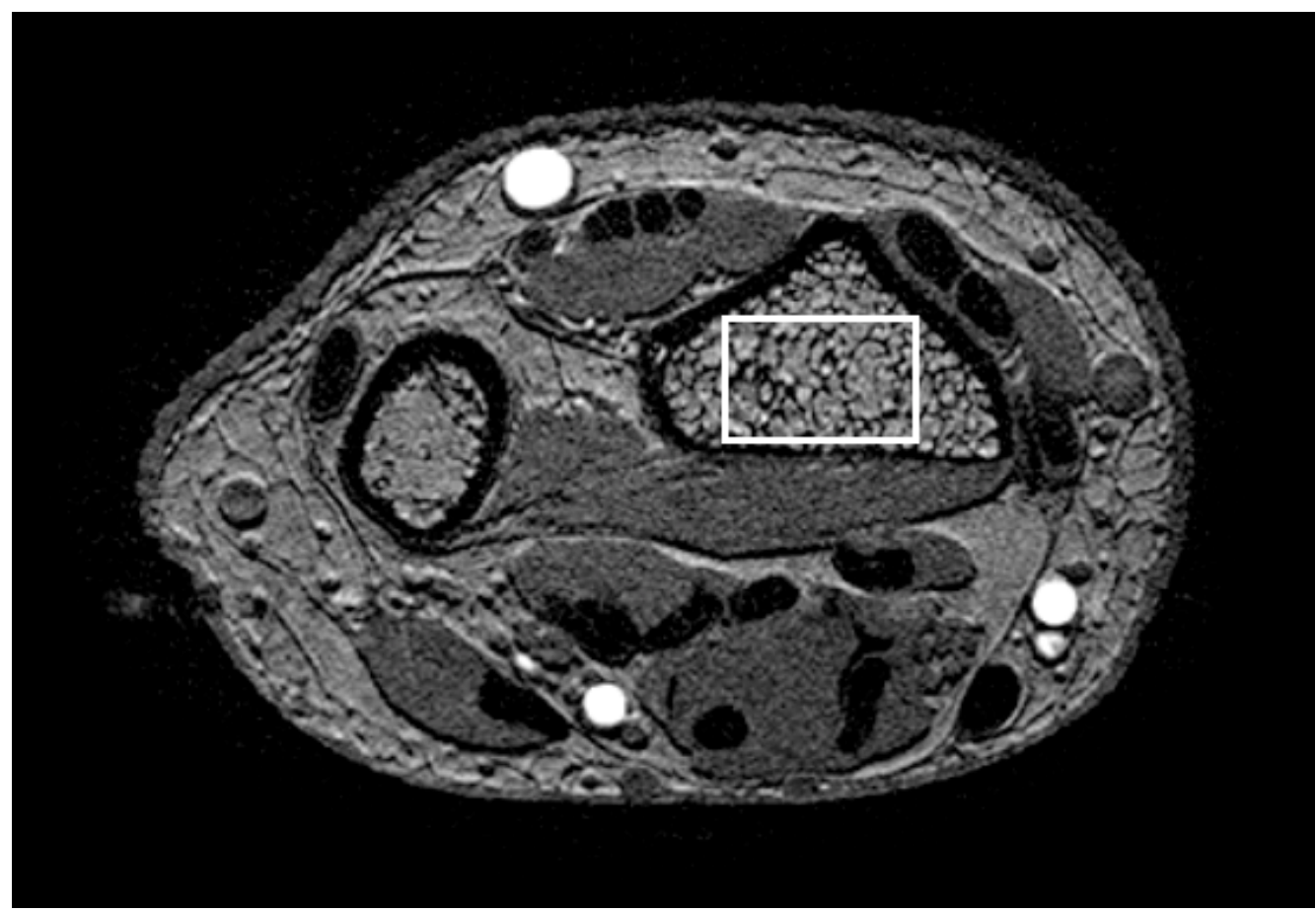

Figure 2.10. Semi-automated segmentation by the selection of a rectangular ROI in the most proximal slice. Rectangular region is propagated through the rest of the slices by direct projection.

An example of a segmented region can be observed in figure 2.11a.

\subsubsection{Coil heterogeneities correction and bone volume fraction calculation}

For a proper analysis of the trabecular bone network, the appropriate classification (bone or marrow) of the voxels of the MRI images should be ensured. All the processes of post-classification analysis will be affected by its outcome.

Due to the high spatial resolution used in acquisition, not common in clinical MRI acquisitions, slight modulations of the signal intensities across the acquisition volume, also known as coil shading phenomena, appeared due to small local magnetic field heterogeneities. This can cause the voxels to be classified incorrectly if two voxels with the same content (i.e. bone) located in different regions of the image have different intensity values.

Thus, it was decided to apply nearest-neighbor statistics by the implementation of a 3D local thresholding algorithm (LTA), as a generalization from its 2D 
version [78]. It consisted in the calculation of the Laplacian on different regions of the volume in order to obtain a threshold intensity value.

Marrow intensity values in the neighborhood of each voxel were determined and bone voxels intensities were scaled using calculated local intensities. Concretely, the method is based on the calculation of the average Laplacian values $\langle\tilde{L}\rangle_{\vec{r}}(I)$ in a sphere region $S(\vec{r})$ with a radius $\mathrm{R}=15$ pixels, being $I$ the voxel intensity and $\vec{r}$ the center of the sphere, which is displaced through all voxels of the volume. When the calculated Laplacian equals zero $\langle\tilde{L}\rangle_{\vec{r}}\left(I_{t}(\vec{r})\right)=0$, the corresponding marrow intensity $I_{t}(\vec{r})$ can be determined locally. After the marrow intensity is obtained, it is used as a threshold and voxels can be directly classified into pure marrow voxels or scaled by its local threshold value and labeled as partially occupied by bone voxels [78].

Finally, a map of local intensities thresholds are obtained, which are used to adjust the amount of bone in each voxel, that is, equalize or normalize the grayscale values of image voxels. An example can be observed in figure $2.11 \mathrm{~b}$.

\subsubsection{Sub-voxel processing}

The extreme conditions in terms of low SNR and partial volume effects due to larger voxel size than typical thickness of the trabeculae, which is about 100$150 \mu \mathrm{m}$, forced the implementation of a method to increase the reconstructed spatial resolution.

A subvoxel-processing algorithm was implemented to minimize partial volume effects and to improve the cancellous bone structural quantification from MRI [79]. The method consisted in a two-pass algorithm where each voxel was initially divided into eight subvoxels which are assigned a level of intensity conditioned by the corresponding level of their voxel and near subvoxels, and also under the assumption that the amount of bone intensities must be conserved. In the first pass of the algorithm, each subvoxel was assigned an 
intensity value depending on the intensities of the adjacent voxels and the local sum of intensities. The second pass of the algorithm consisted on the refinement of the previously calculated subvoxel intensities considering the intensities of the neighboring subvoxels and the total sum of intensities conservation [79]. Finally, an increased apparent isotropic spatial resolution of $90 \mu \mathrm{m}$ was achieved and partial volume effects were minimized (Figure 2.11c).

\subsubsection{Binarization}

Finally, at the end of the image processing chain, the resulting images were binarized into exclusively bone or marrow voxels (see Figure 2.11d).

Histogram shape-based thresholding was implemented through the Otsu's method developed in 3D [80]. The method consists in the minimization of the intra-class variance of volume intensities, which has been shown to be equivalent to maximizing the between-class variance. Thus, the optimum separation threshold was calculated for the entire volume using:

$$
t^{*}=\arg \cdot \max _{t} \frac{\sigma^{2} B}{\sigma_{T}^{2}}
$$

where $\sigma^{2}$ is the total variance and $\sigma^{2}{ }_{B}$ is the between-class variance. The optimum binarization threshold is $t^{*}$. Finally, voxels were classified as bone or marrow depending on their intensity value.

A 3D logical matrix containing the bone structure $\left({ }^{\prime} 1\right.$ '=bone, ' 0 '=marrow) resulted from the application of the binarization process. 

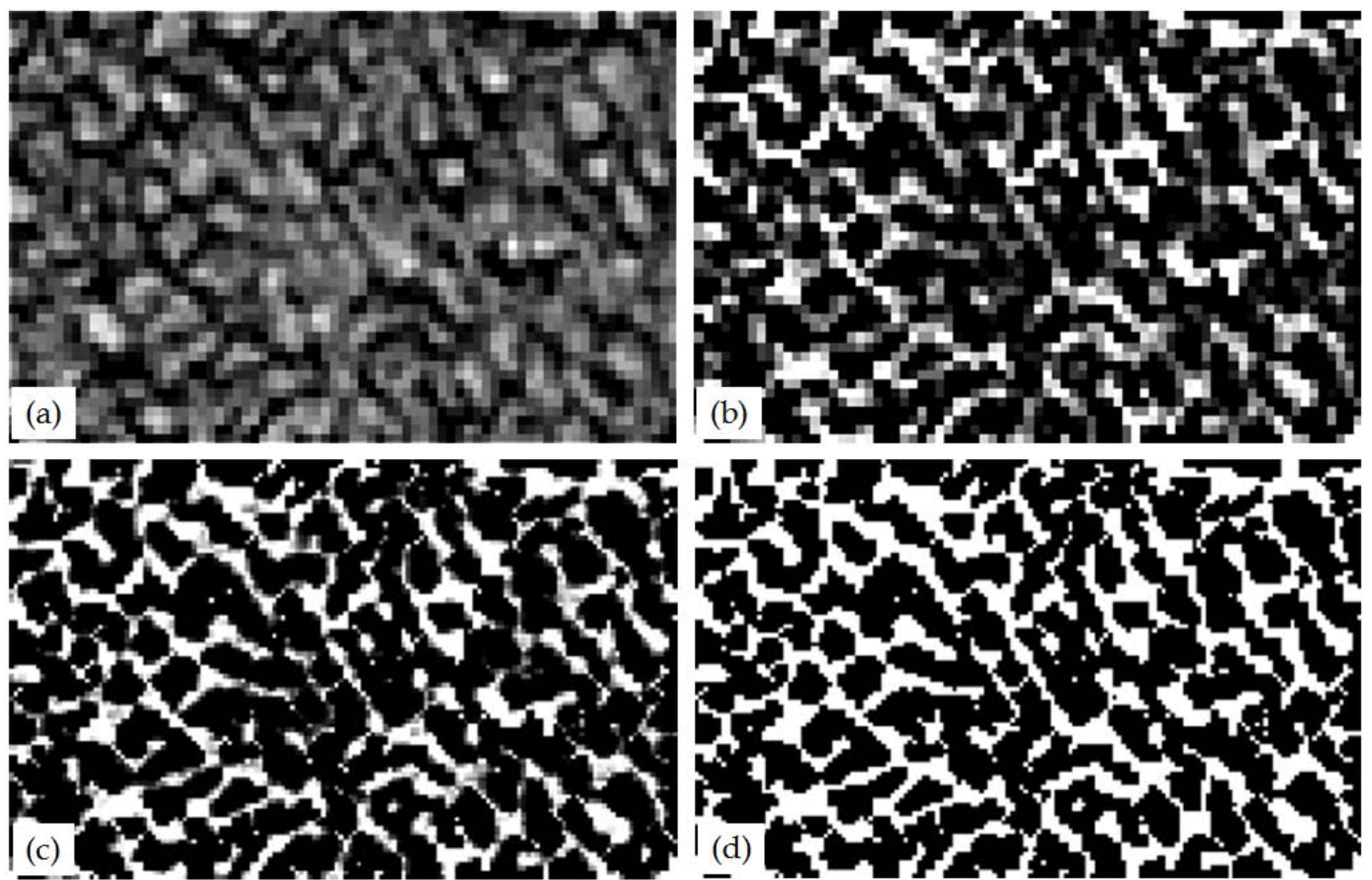

Figure 2.11. In (a), a segmented region containing exclusively bone (dark) and bone marrow (bright). In (b), synthesized map showing the amount of bone in each voxel. The intensity of the voxel is proportional to the bone quantity. In (c), interpolated image after the execution of the sub-voxel processing algorithm for an increased spatial resolution. In (d), final binarized image containing exclusively bone or marrow voxels.

\subsection{D reconstructions of the trabeculae}

The 3D volumetric reconstructions provide us with visual information about the microarchitecture of the different structures under study. For its graphical representation, a simple plane superimposition can be directly performed. However, for improved rendering quality, triangulation algorithms in combination with isosurfaces extraction and lighting effects may be applied.

The primary implementation of the algorithm for the 3D reconstruction of the trabecular bone was performed by overlapping the slices in the three space dimensions. This reconstruction procedure consists in using the orthogonal plane 2-D texture mapping technique. An example of 3D reconstruction based in this method is shown in figure 2.12 . 


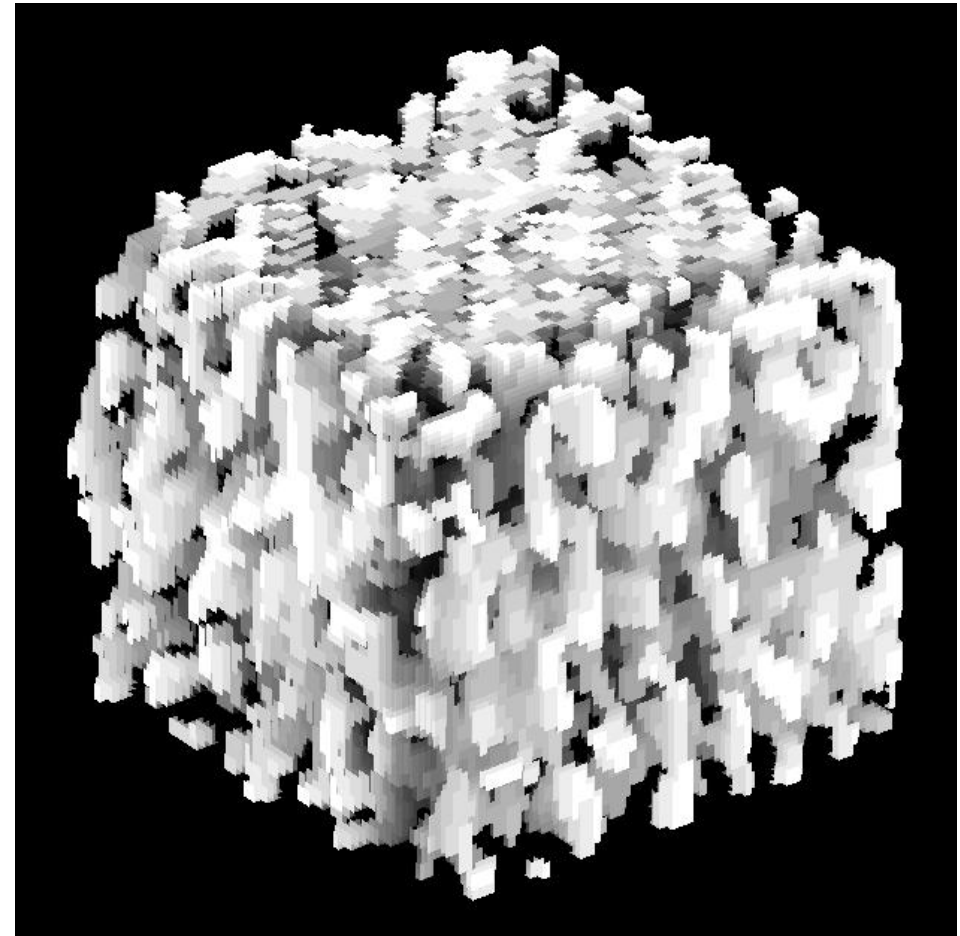

Figure 2.12. Reconstructed 3D microarchitecture of a trabecular bone sample using the orthogonal plane 2-D texture mapping.

However, an improved rendering of the trabecular bone samples can be performed by the initial application of an algorithm for Delaunay tessellation of the extracted structure [81] (figure 2.13a). This description of the geometry is made from small triangles that define the surfaces. Each triangle forming the trabecular bone microarchitecture is defined by its vertices and connectivity. These triangles should fit each other perfectly and without gaps. The smoothness of the 3D reconstruction is given by the size of the used triangles.

If triangle faces are colored and a light focus is added to some point of the 3D space, the structure visualization is significantly enhanced, as it can be appreciated in figure $2.13 \mathrm{~b}$. 

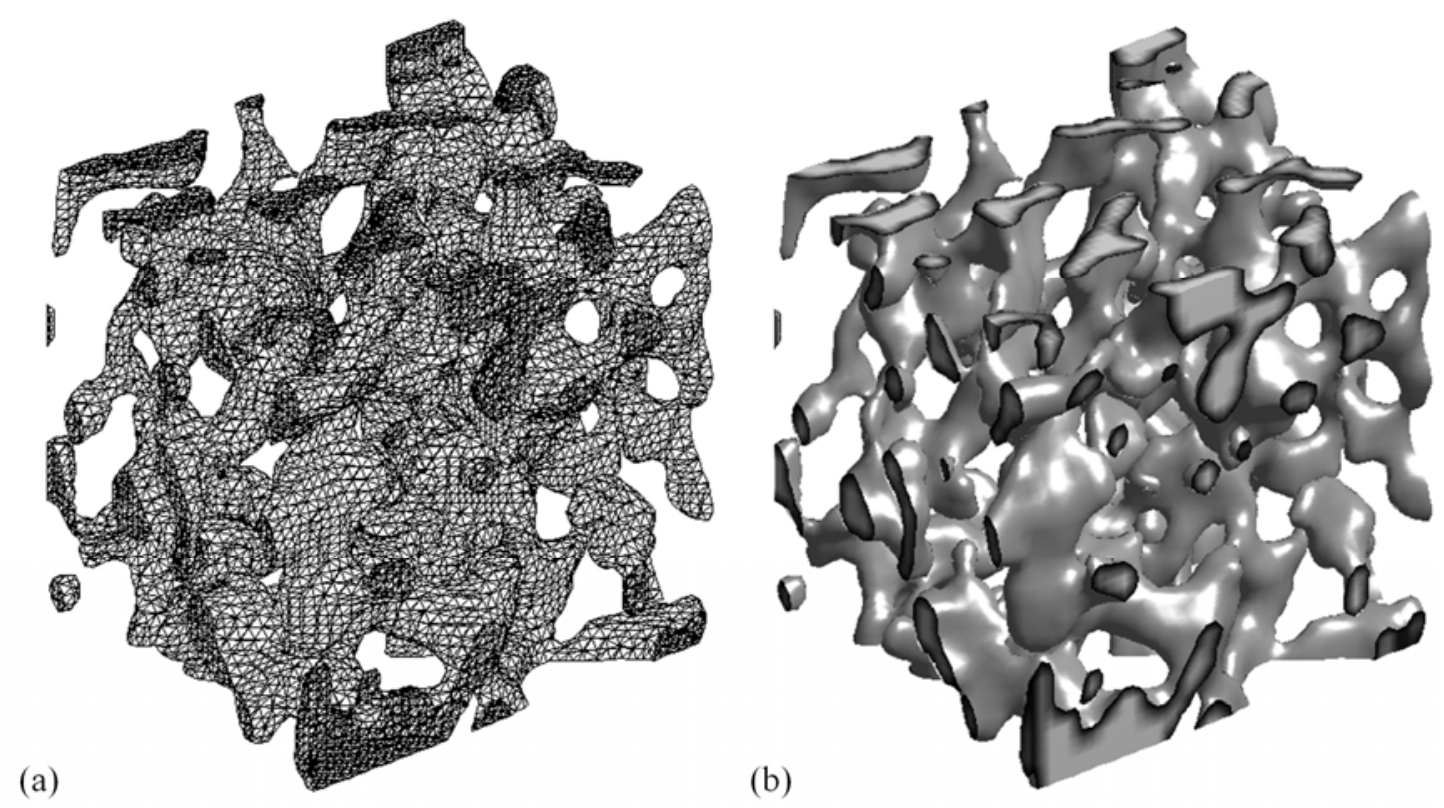

Figure 2.13. Rendered 3D reconstruction of the trabecular bone. In (a), triangulated geometry of the trabecular bone structure after Delaunay tessellation. In (b), final volume rendering after the inclusion of color and a light source to the geometry.

This rendered data can be directly built into a rapid prototyping stereolithography file in STL (Standard Tesselation Language) since the output data of the Delaunay triangulation algorithm is in a similar format.

The STL is the most common format for geometry exchange and it is also widely used in industry since it is considered as a standard for rapid prototyping. Any geometry that is defined in STL can be physically manufactured by industrial lithography systems to obtain a prototype [82].

There are two types of STL file, corresponding to the data style, which can be either in binary or ASCII format. The binary STL files are difficult to implement but they suppose small file sizes. On the contrary, ASCII is characterized by its ease of implementation while its major drawback is the increased file size.

In an ASCII file, each triangle is defined as follows: 


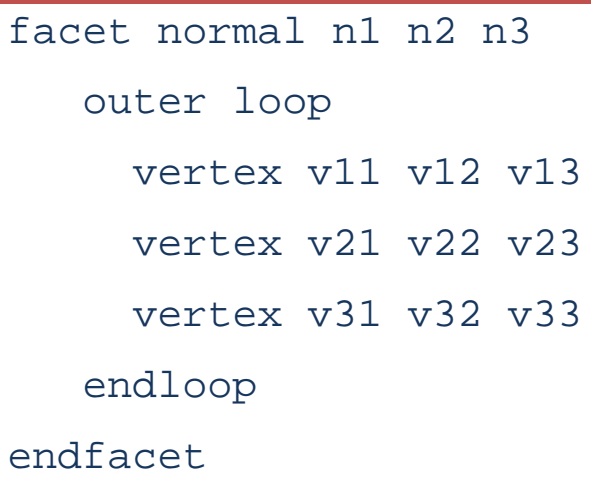

Where $n 1, n 2, n 3$ represent the components of a unitary vector which is normal to the plane defined by the triangle vertices. The command 'vertex' is used to define the coordinates of each one of the three triangle vertices. A routine to export the geometries of the $3 \mathrm{D}$ reconstructions of the trabecular bone to the well extended ASCII STL format was finally implemented.

\subsection{Morphometry analysis}

In this section, the methods developed and implemented to quantify the most significant structural parameters of the cancellous bone are deeply explained. Morphometry analysis is based on the determination of parameters regarding physical static properties like structure dimensions and shape, architecture complexity, elements topology and anisotropy of the trabeculae arrangement.

\subsubsection{Morphology}

A structural analysis was developed on the 3D structure to characterize the morphological properties of the cancellous bone [76, 83]. The calculated parameters were the relationship between the bone volume and the total volume under analysis, $(B V / T V)$ also known as trabecular volume, mean trabecular thickness (Tb.Th), mean trabecular bone separation (Tb.Sp) and a trabecular index which relates different morphology parameters, trabecular number (Tb.N). 


\subsubsection{Bone volume to total volume percentage $(B V / T V)$}

To represent the percentage of bone voxels in the whole segmented volume, $B V / T V$ was calculated by dividing the number of bone voxels of the $3 \mathrm{D}$ reconstruction by the global number of elements, as it can be directly deduced from equation 2.2:

$$
B V / T V=\frac{n_{-} \text {bone _ voxels }}{n_{-} \text {total_voxels }}
$$

Results are expressed as a percentage (\%).

\subsubsection{Mean trabecular bone thickness $(T b . T h)$}

The $T b . T h$ represents the mean thickness value of all the beams of trabecular bone of the 3D structure. First, a 2D skeletonization algorithm was applied to each slice and pixels labeled as trabeculae axis selected. Pixels corresponding to the boundary of the trabeculae were also detected by the application of a contour detection algorithm. Then, the distance transform method [84] was applied to the contour image to obtain the minimum distance from each pixel to the contour (see figure 2.14). Measurements are expressed in $\mu \mathrm{m}$. 


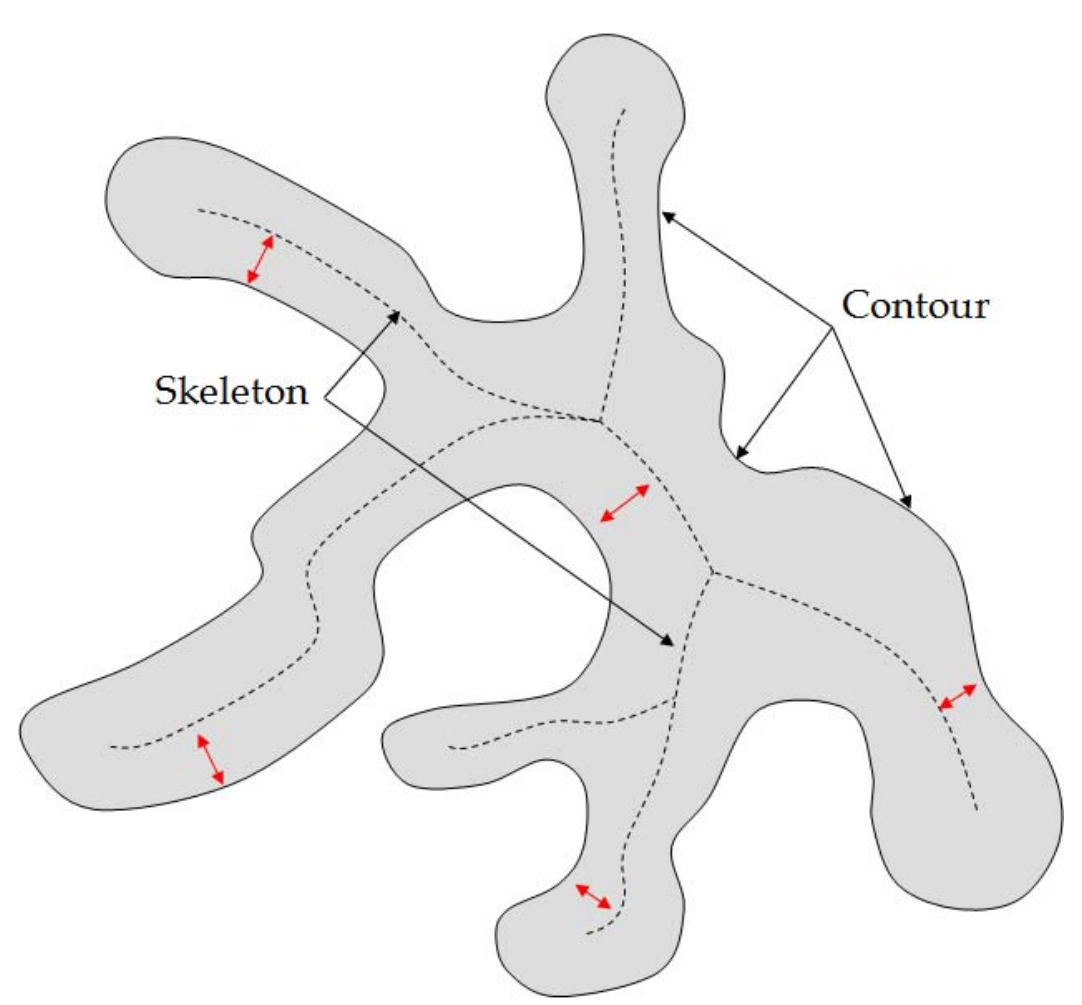

Figure 2.14. Iconography to explain the basis of the method for the Tb.Th calculation. The distance transform is applied to the extracted contours. Then, the minimum distance between each pixel of the skeleton to the trabecular contour is obtained and multiplied by 2 to obtain the local trabecular thickness.

The skeletonized image was multiplied by the resultant of the transform distance to obtain the minimum distance from each voxel of the skeleton to the boundary, that is, the half of the trabecular thickness. Mathematically, the expression applied for the calculation of the $T b$.Th is given in equation 2.3.

$$
T b . T h=\frac{\sum_{c} \frac{\sum_{p \in S k} 2 \cdot d_{\min }}{|S k|} \cdot \rho}{|c|}
$$

where $d_{\min }$ is the minimum distance from a pixel of the skeleton to the trabecula contour, $p$ is any pixel belonging to the skeleton $(S k), c$ is each slice of the reconstruction, and $\rho$ the spatial resolution of the image.

A detailed diagram of the process for the calculation of the trabecular bone thickness in a given binarized slice is shown in figure 2.15. 


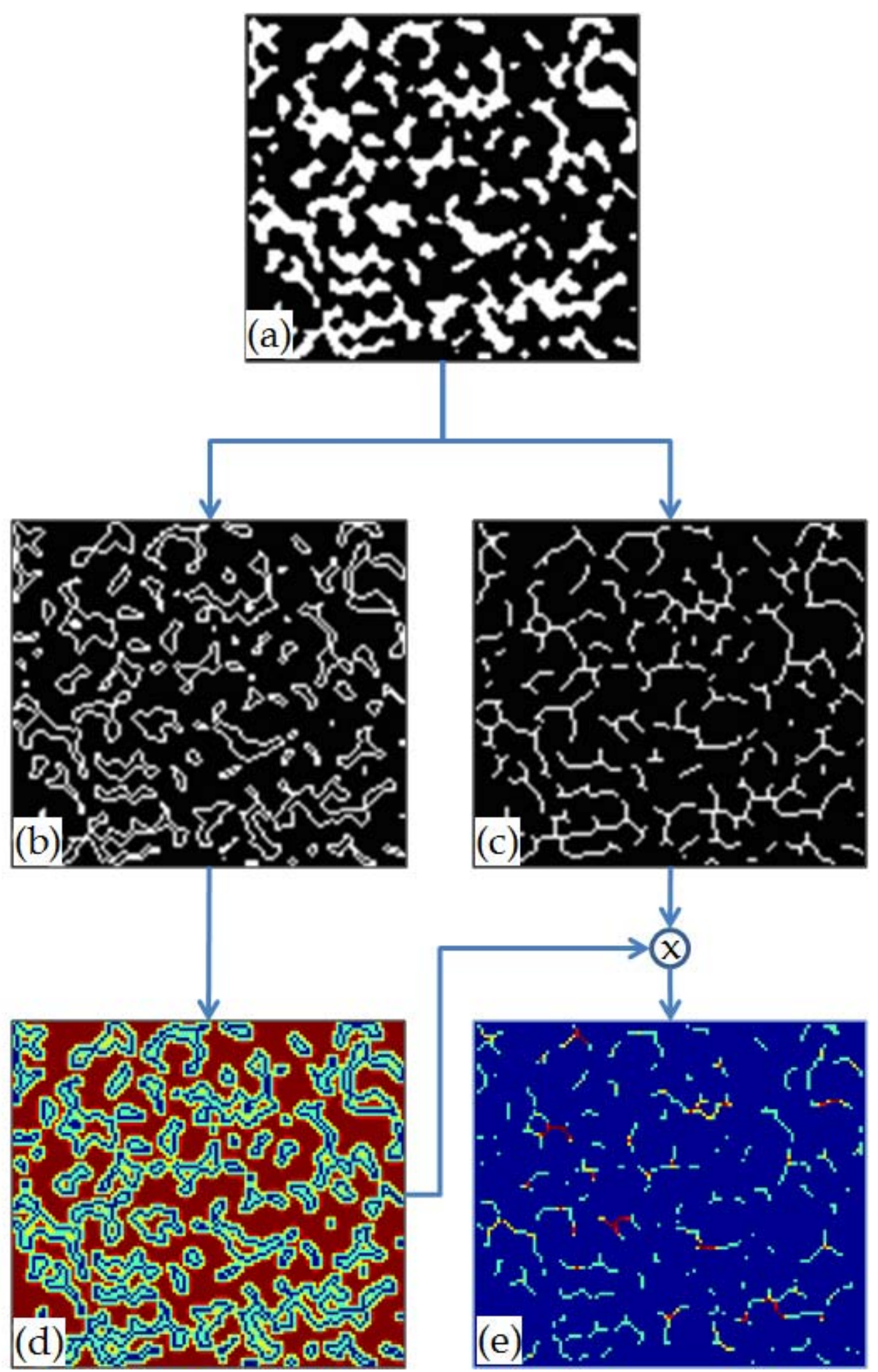

Figure 2.15. Image processing methods for the estimation of the trabecular bone thickness of a given slice (a). The reconstructed slice (a) is initially divided into its contours (b) and skeleton (c) structures. If the distance transform is applied to the contours image, the minimum distance of each pixel of the image to the contour is obtained (d). Finally, the calculated distance transform to the contours is multiplied by the image skeleton to obtain the minimum distance of each skeleton pixel to the contour (e). 


\subsubsection{Mean trabecular bone separation (Tb.Sp)}

The Tb.Sp is defined as the mean size of the intertrabecular spaces or pores. It is calculated by counting the mean number of contiguous marrow voxels in each one of the three space directions. The different measurements are averaged and the Tb.Sp calculated. However, due to the pore sizes heterogeneity, it is common to obtain large standard deviations (SD) in Tb.Sp and it is also needed to store the pore size distribution obtained from the Tb.Sp analysis. Measurements are usually expressed in $\mu \mathrm{m}$.

\subsubsection{Trabecular number (Tb.N)}

The Tb.N gives an idea of the percentage of trabecular bone present in the volume against the mean trabecular thickness. Its calculation is straightforward by equation 2.4 [85].

$$
\text { Tb.N }=\frac{B V / T V}{T b . T h}
$$

Units are expressed as $\mu \mathrm{m}^{-1}$.

\subsubsection{Complexity}

The $2 \mathrm{D}$ and $3 \mathrm{D}$ approaches for the in vivo fractal characterization of the trabecular bone reconstructions were implemented. In order to calculate the trabecular bone $D^{2 D}$, the conventional box-counting algorithm was implemented. A slice-by-slice contour detection routine was included at the beginning of the code. For each slice, grids of different sizes were sequentially overlaid to the contour image, as it can be visualized in figure 2.16. The total amount of grid boxes containing boundary pixels was calculated for each box size and stored in a vector. 

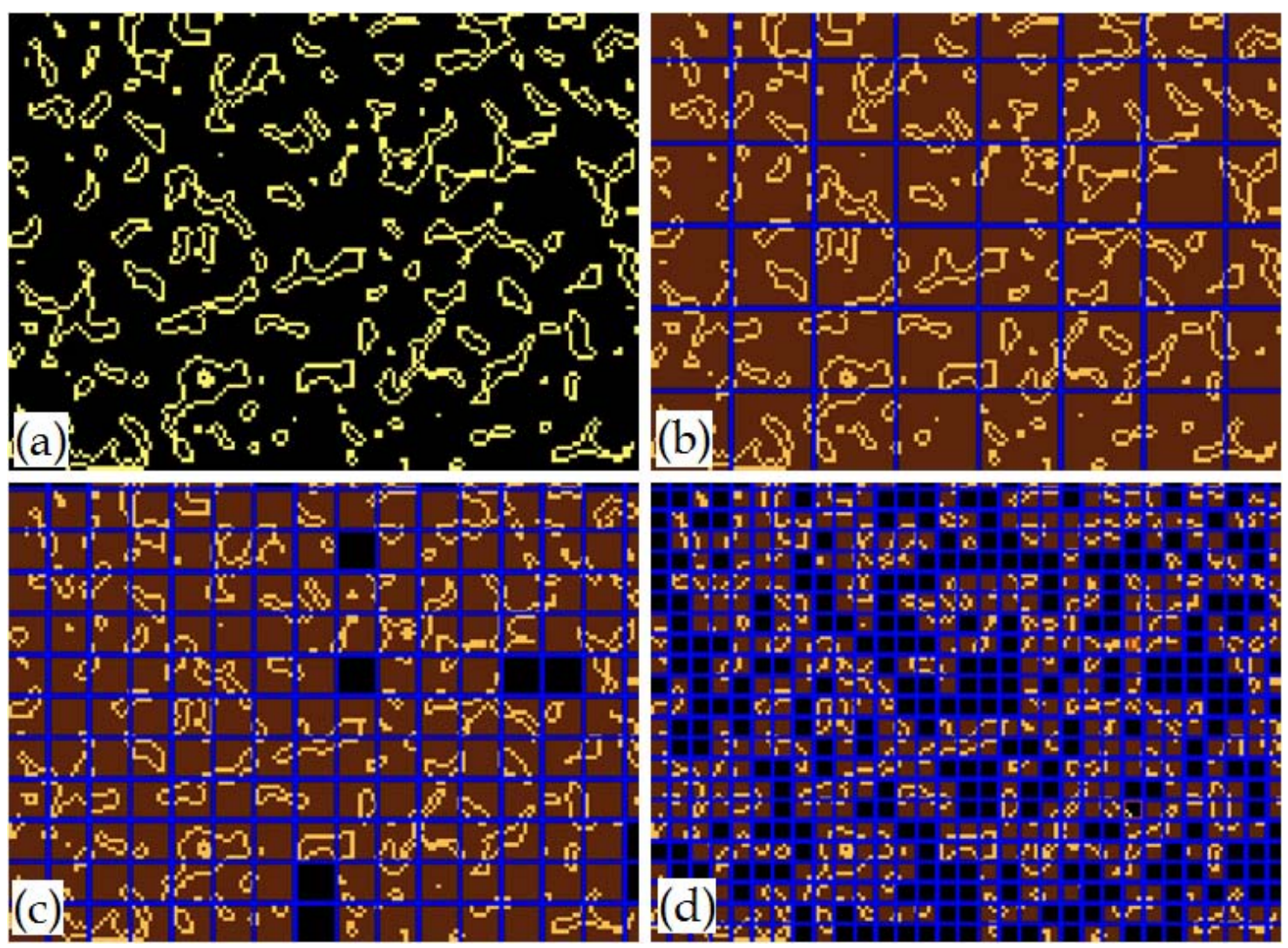

Figure 2.16. Trabecular bone contour detection after image processing steps can be observed in

(a). Grid of 20 pixels size superimposed on the trabecular bone isolated region. All boxes

contain bone contour, (b). Superimposing of a grid of 10 pixels size to the trabecular bone

contours image. Six boxes are not containing trabecular bone contour, (c). Small grid

superimposing of 5 pixels size to the image of the trabecular bone contours. A high number of boxes are not containing contour, (d).

Once the number of boxes containing contour was obtained for each grid size, data was fitted by least mean squares according to:

$$
\log (N)=-D^{2 D} \cdot \log (\lambda)+k
$$

Equation 2.5 gives the relationship between the number of contour boxes $(N)$, the corresponding box size $(\lambda)$, the box-counting fractal dimension parameter $\left(D^{2 D}\right)$ and a proportionality constant $(k)$. Geometrically, $D^{2 D}$ corresponds to the negative value of the slope of the line relating the natural logarithms of the number of boxes containing contour and each corresponding box sizes. The fractal dimension was calculated for each slice and results were averaged in 
order to calculate the $D^{2 D}$ parameter for each scan. The $2 \mathrm{D}$ box-counting algorithm implemented for the quantification of 2D fractal complexity was validated by its application to the Sierpinski triangle figure which has a known fractal dimension of $\log (3) / \log (2)=1.585$. Result of the validation can be appreciated in figure $2.17 \mathrm{a}-\mathrm{b}$.

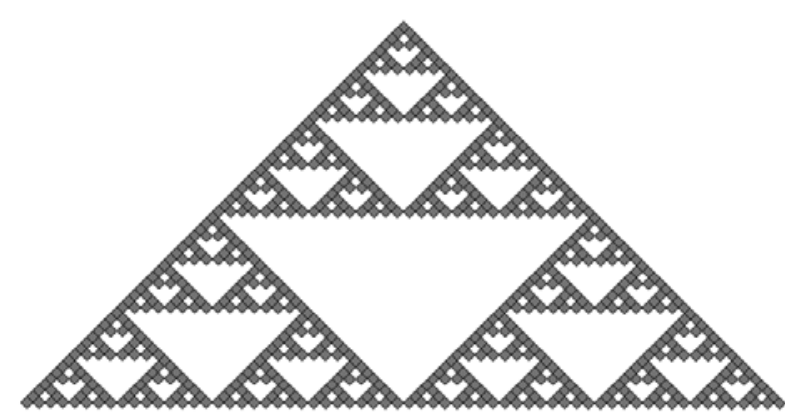

(a)

(c)

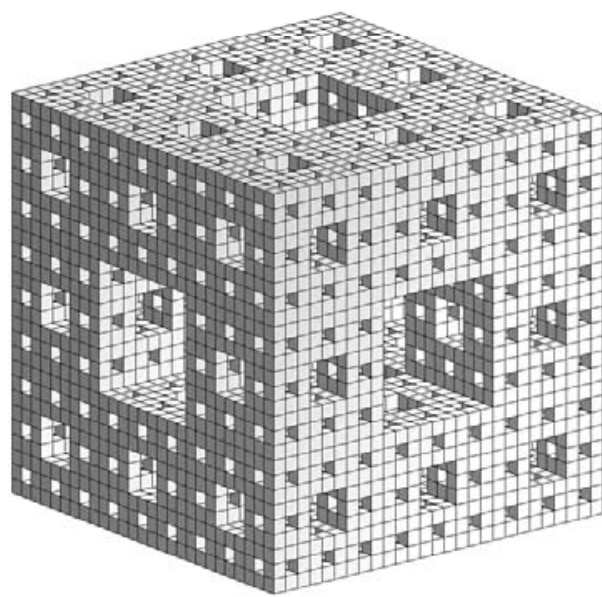

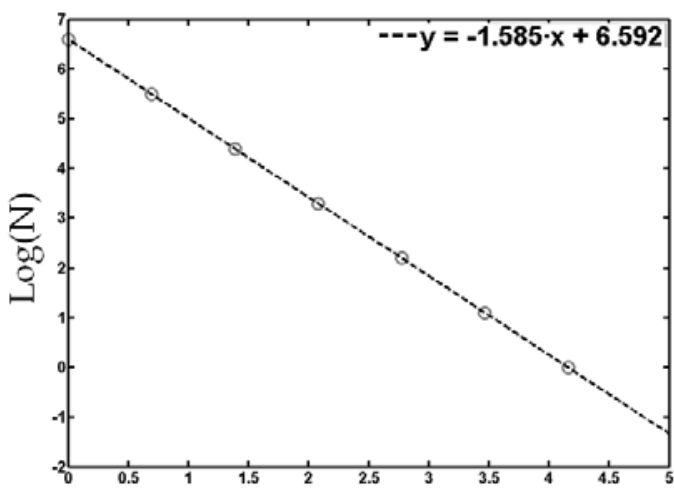

(b)

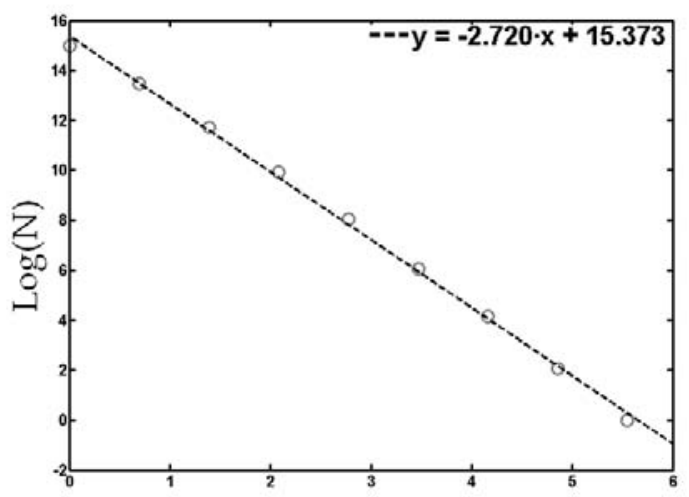

(d)

$\log (\lambda)$

Figure 2.17. Validation of the 2D and 3D fractal dimension calculation methods. Sierpinski triangle synthesized for the validation of the 2D methodology (a). Calculated points and fitted slope corresponding to the negative value of the $D^{2 D}$ parameter $(\mathrm{b})$. Menger sponge synthesized for the validation of the 3D methodology (c). Calculated points and fitted slope corresponding to the negative value of the $D^{3 D}$ parameter (d).

A generalized version of the box-counting algorithm was also developed and implemented for the quantification of the 3D trabecular bone complexity. The box-counting algorithm previously used for 2D calculations was herein extrapolated to the 3D space domain. As an original work, trabeculae surfaces for 3D fractal analysis were computed by Delaunay tessellation [81] in 
combination with the marching-cubes algorithm [86] before the application of the $3 \mathrm{D}$ box-counting method, as it may be observed in figure 2.18.

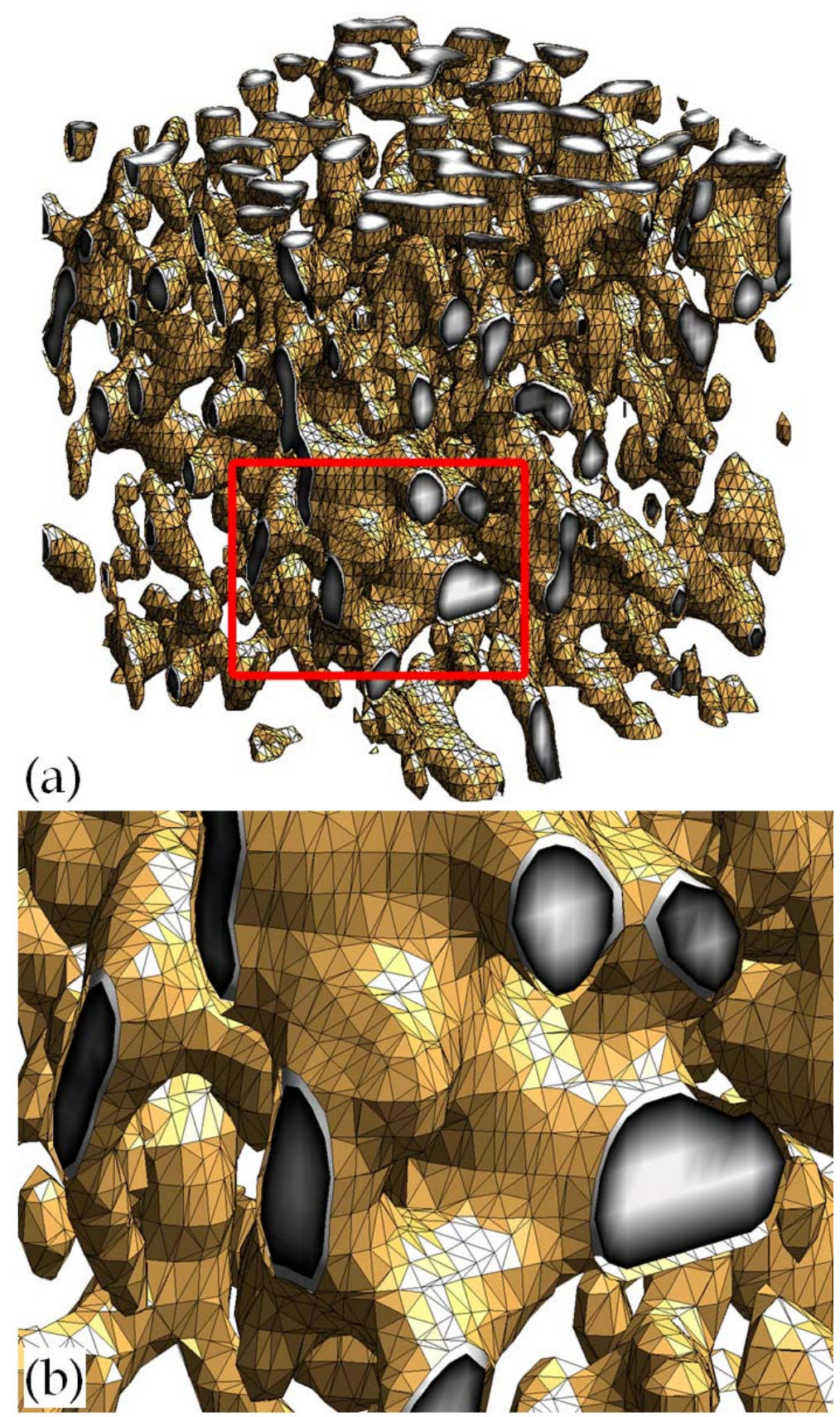

Figure 2.18. 3D reconstruction of the trabecular bone with surface extraction after the application of the marching cubes algorithm (a). Zoomed region where a good depiction of the extracted surface and inner volume can be observed (b).

Different 3D cube meshes with varying cube sizes were generated and mixed with the $3 \mathrm{D}$ reconstruction of the trabecular bone surfaces. Analogously, the number of cubes containing trabecular surface were calculated for each cube 
size and stored in a vector. Cube size dimensions were generated similarly to the 2D method and the maximum cube size was the smallest power of two over the largest dimension of the $3 \mathrm{D}$ surface matrix. The $D^{3 D}$ parameter was directly calculated by least mean squares fitting of the data to Equation 2.5. The 3D boxcounting method implemented was also validated by its application to the Menger sponge structure, which is characterized by a theoretic fractal dimension of $\log (20) / \log (3)=2.727$. Result of the validation can be appreciated in figure $2.17 \mathrm{c}-\mathrm{d}$.

\subsubsection{Topology}

The basis of digital topological classification (DTA) relies on a categorization of each bone voxel of the 3D trabecular network into different groups depending on their localization or structural role in the microarchitecture. The method is based on determining the connectivity of the neighbors in the $3 \times 3 \times 3$ neighborhood of the examined voxel [69].

The starting point of the analysis is the conversion of the $3 \mathrm{D}$ network to a skeletonized surface representation, which contains only 1D and 2D structures only, that is, rods and plates, that can be expressed as curves and surfaces. The voxels are then assigned to the appropriate topological class (e.g., curves, surfaces, or junctions) in a three-step approach commented ahead.

\section{Skeletonization}

Although a fast 2D simple skeletonization is performed previously before the 2D distance transform which is needed for the trabecular thickness calculation, it is not related to the implemented 3D skeletonization algorithm being described in this section, which is exclusively required to prepare the structure for topology analysis. 
The objective of 3D skeletonization is to produce a medial surface representation that preserves the topology and maintains the shape of the object as much as possible [87].

The skeletonization process consists in iterative erosion which is divided in two steps:

- Primary skeletonization, where a primary and non-refined skeleton is obtained.

- Final skeletonization, whose result is an object that is the final skeleton and is compound exclusively by $1 \mathrm{D}$ and $2 \mathrm{D}$ elements (curves and surfaces).

Given a certain voxel, the basic adjacency relationships used for the skeletonization algorithms is shown in figure 2.19 .

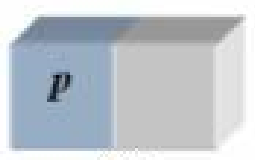

(a)

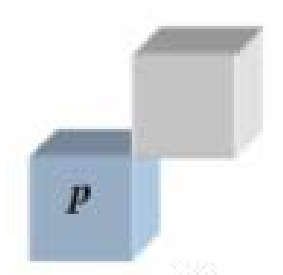

(b)

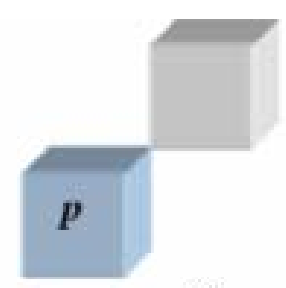

(c)

Figure 2.19. In gray, different localizations for the neighbors of a voxel p: s-point (a), e-point (b) and v-point (c).

The neighborhood voxels can be classified according to their adjacency to the $p$ voxel as:

- S-point: 6-adjacent neighbor to $p$ and, therefore, adjacent to each side.

- E-point: 18-adjacent neighbor but not 6-adjacent to $p$ and, therefore, adjacent to at edge.

- V-point: 26-adjacent neighbor but not 18-adjacent to the $p$ and, therefore, adjacent per vertex.

More complex adjacency definitions need to be defined in order to maintain the shape of the structure while preserving the topology information [87].

The primary skeletonization is an iterative process that terminates in the iteration in which no items are deleted, that is, if at iteration ' $i$ ' any voxel is removed, the iteration will continue to ' $i+1$ '. Each iteration of the process is 
divided into three successive scans. Each scan is focused on labeling s-point voxels, e-point voxels and v-point voxels, respectively.

The final skeletonization is based on a non-iterative process where voxels classified as erodable-points are removed [87]. In the end, a 3D skeletonized structure preserving topology and original shape is obtained. In figure 2.20, a example of the skeletonization algorithm application to a synthetic structure proposed by Saha et al. in order to validate their method [87].

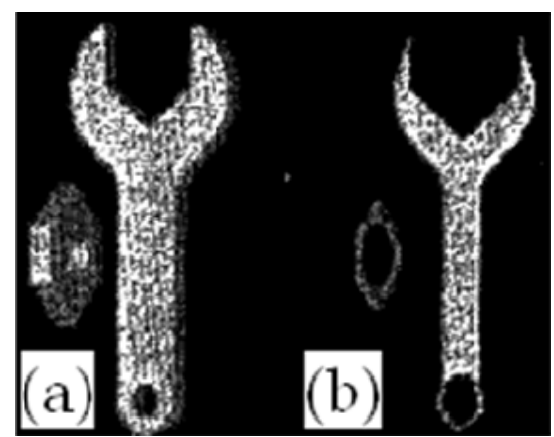

Figure 2.20. In (b), result of the skeletonization process applied to a synthesized wrench (a) [87]. Note that 3D information has disappeared in the skeletonized version and also that main shape and topology is maintained.

In order to validate the implementation of the skeletonization algorithm, during the development process, small size arrays were used to validate each one of the steps for skeletonization. After the final implementation of the skeletonization algorithms, a structure similar to the one shown in figure 2.20 was generated in order to validate the method. A black and white image of the wrench was created in $2 \mathrm{D}$ and then the array was concatenated in the third dimension, with aim of achieving a three dimensional object (see figure 2.21).

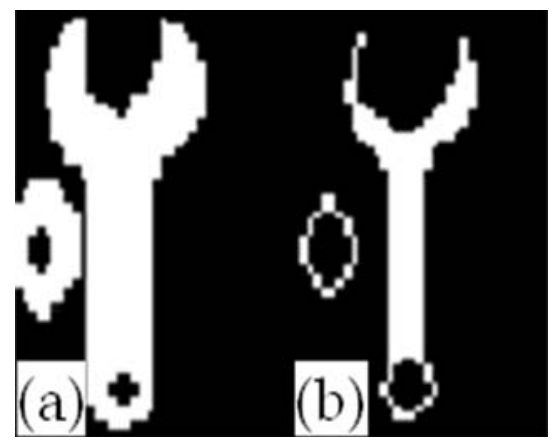

Figure 2.21. In (a), synthetic image that simulates figure 2.20a. In (b), result after applying the skeletonization algorithm to (a). A strong similarity to the results obtained with the model presented in figure 2.20 are obtained. 
Finally, the implemented skeletonization algorithm is applied to binarized 3D trabecular bone reconstructions resulting from the image processing chain explained in section 2.2. An example of the application of the skeletonization routine to a trabecular bone reconstructed sample can be observed in figure 2.22 .
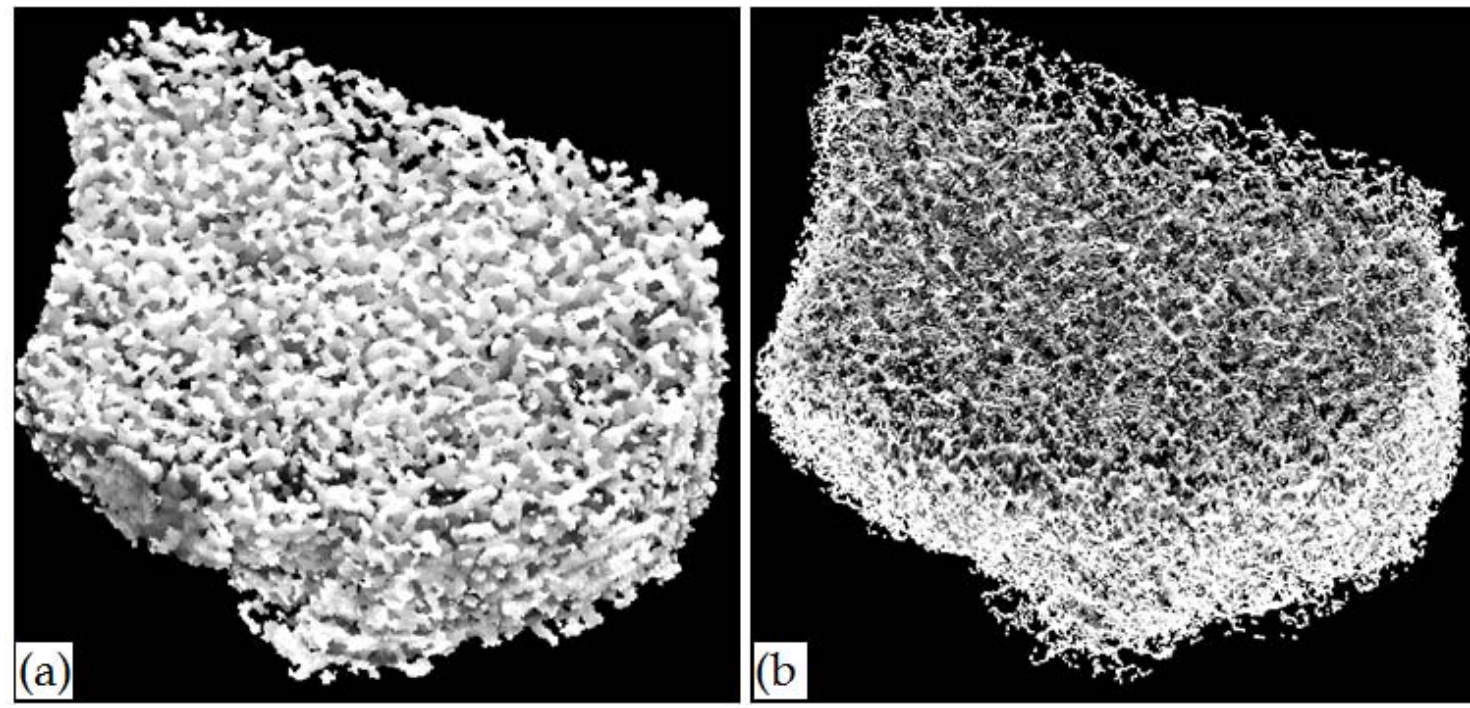

Figure 2.22. A 3D trabecular bone reconstruction before the application of the skeletonization algorithm (a). Skeletonized 3D trabecular bone reconstruction containing only 1D and 2D structures (curves and surfaces) (b).

$\underline{\text { Topological analysis }}$

The topological analysis of the cancellous bone structure is based on the study of the connectivity of the $3 \times 3 \times 3$ neighborhood of each voxel of the 3D skeletonized reconstruction. The voxels are classified initially under three broad topological classes: surfaces, curves and junctions. Each of the three classes is already subdivided into different subclasses that allow a refinement of the process. The algorithm consists of three steps [76]:

1. Initial topological classification of the voxels, in which the voxels are classified partially and present ambiguities. 
2. Second topological classification of the voxels, which eliminates the ambiguities of the previous classification.

3. Corrections to the classification at critical regions of the structure and detection of profile elements.

As mentioned, the aim of the topological classification is to classify each voxel of the 3D structure-based classes as 2D structures (curves and surfaces). Table 2.2 shows the topological classes to consider the spatial interpretation.

\begin{tabular}{|c|c|}
\hline Class & Description \\
\hline S-Type & Surface Interior \\
\hline SS-Type & Surface-Surface junction \\
\hline SE-Type & Surface Edge \\
\hline SC-Type & Surface-Curve junction \\
\hline C-Type & Curve interior \\
\hline CC-Type & Curve-Curve junction \\
\hline CE-Type & Curve Edge \\
\hline PE-Type & Profile Edge \\
\hline I-Type & Isolated \\
\hline
\end{tabular}

Table 2.2. Different topological classes in which voxels resulting from the 3D skeletonization are categorized

The classification is performed by considering the definitions of the different classes that must be accomplished by each voxel in order to belong to a particular class. Gomberg et al. made a detailed explanation of the different conditions used for the decision algorithm, including useful mathematical formal demonstrations [70].

The topological classification is mainly based on the analysis of the adjacency relationships in a voxel-by-voxel basis. A voxel can have six adjacent voxels per side and 26 adjacent voxels per edge and side.

Adjacency conditions to be met for the different classes:

- $\quad$ Surface Interior (S): voxel that has at least three adjacent bone voxels per side. 
- Surface-Surface junction (SS): voxel that meets the requirements for a surface edge (SE) voxel, but at the same time is adjacent to one or two $S$ voxels.

- $\quad$ Surface-Edge (SE): voxel that has a side adjacent $S$ voxel, and a side adjacent marrow voxel on the opposite side.

- $\quad$ Surface-Curve junction (SC): voxel that has two adjacent voxels per side, one belonging to the curve $(\mathrm{C})$ class, and other corresponding to the $S$ class.

- $\quad$ Curve interior $(\mathrm{C})$ : voxel that has only two adjacent bone voxels in opposite sides or edges.

- Curve-Curve junction (CC): voxel that has two edge adjacent $C$ voxels and no side adjacent voxels.

- $\quad$ Curve-Edge (CE): voxel that has a $\mathrm{C}$ adjacent voxel and no more adjacent voxels.

- $\quad$ Profile Edge (PE): voxel that has not met the requirements of the previous classes and corresponds to a prominent profile in the continuous topology of a curve.

- $\quad$ Isolate (I): voxel that has no adjacent voxels.

Gomberg et al. implemented a synthetic structure to iconography the different topological classes [70]. Such structure can be appreciated in figure 2.23.

The validation of the implemented DTA algorithm was performed by the generation of a binary structure with an equal voxel arrangement than the observed in figure 2.23. Such structure may be appreciated in figure 2.24a. The result of the validation test can be observed in figure $2.24 \mathrm{~b}$, where all the elements of the structure were classified properly. 


\section{Methodology}

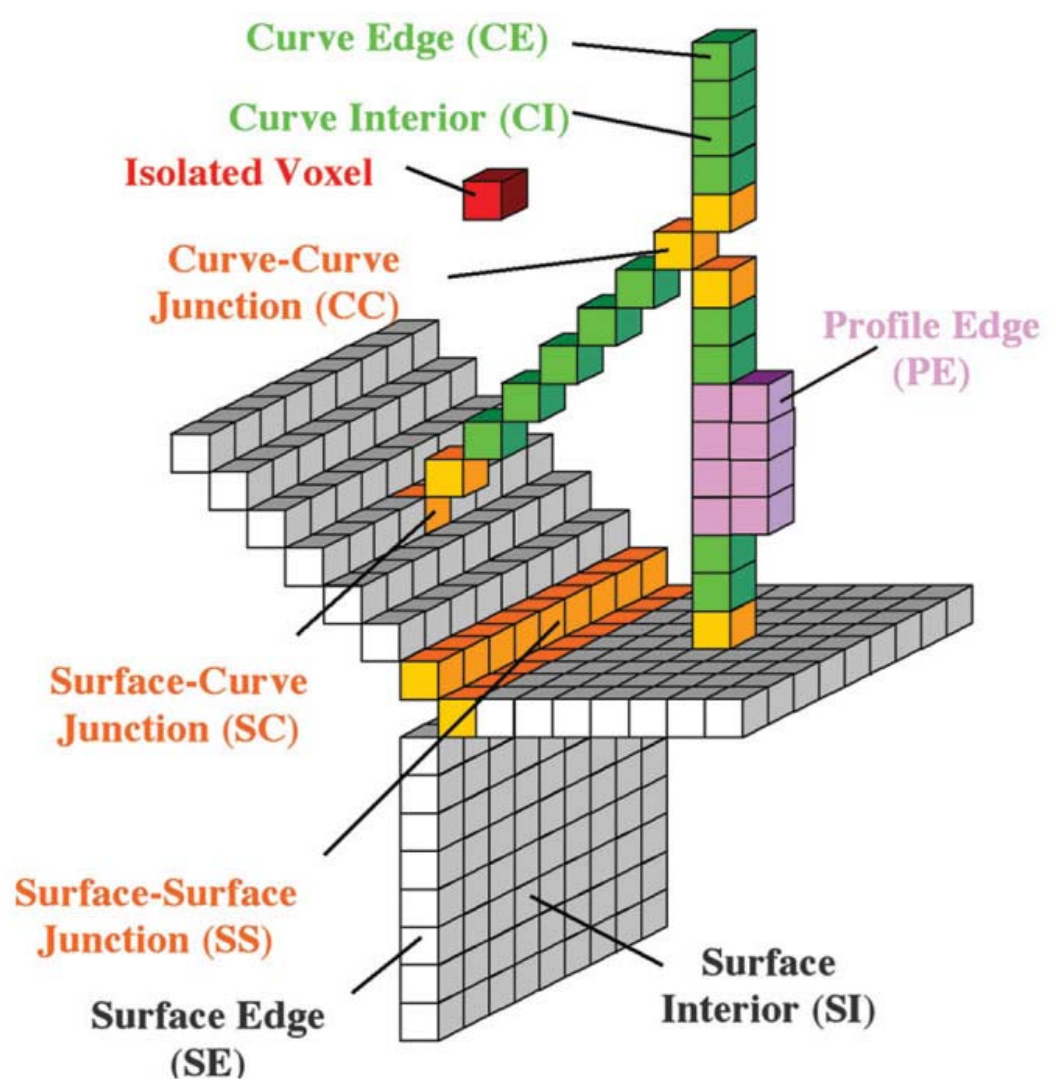

Figure 2.23. Hypothetical digitized structure of portion of trabecular bone after binarization and skeletonization with voxel topological classes indicated [4].

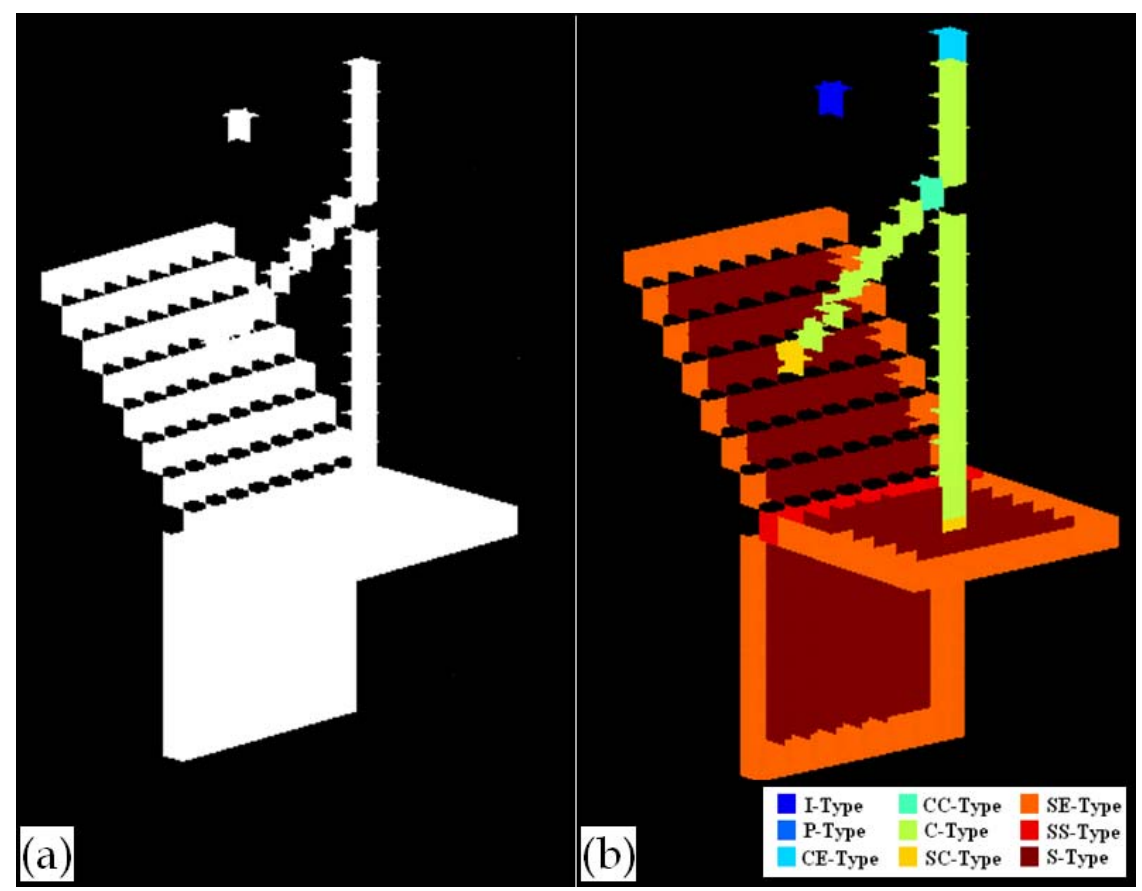

Figure 2.24. Results of the application of the algorithm. A reproduction of the digitized structure proposed by Gomberg et al. can be seen in (a) [70]. Topological classification obtained after the application of the DTA algorithm. 
Finally, the algorithm was applied to 3D trabecular bone reconstructions obtained from in vivo acquisitions. An example of the application of the topological analysis to a small bone sample is depicted in figure 2.25.

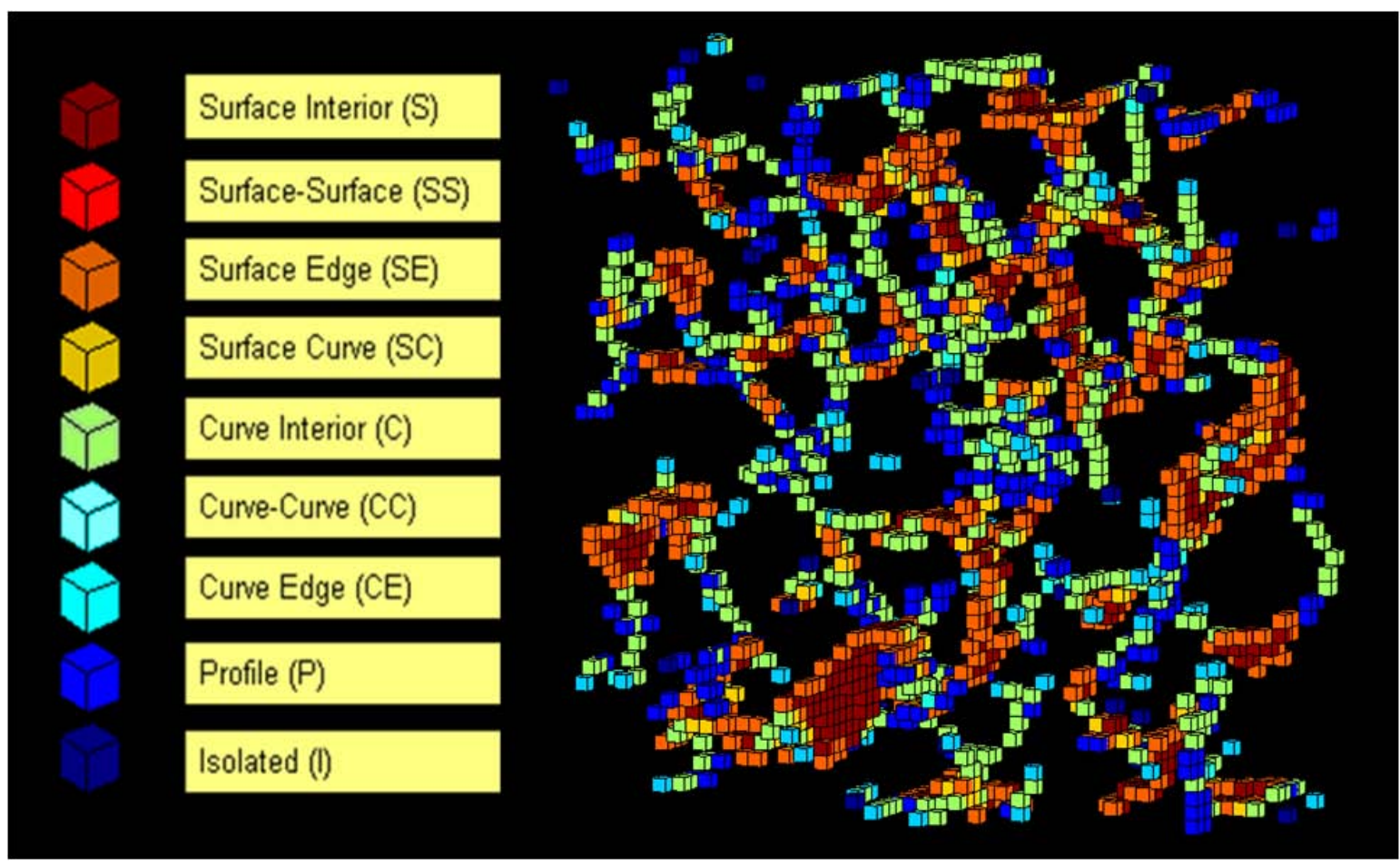

Figure 2.25. Application of the topological classification analysis to a small trabecular bone reconstructed sample acquired from in vivo MR images. Note the trabecular bone plates being represented by surfaces, and rods which have been mainly classified as curves.

Once all the voxels are classified into the specified groups, different topological parameters with high interest for structural bone analysis, such as the total erosion index (TEI) and the surface to curve ratio (SCR) can be calculated [88]. These parameters can be obtained from the relationships between the different voxel quantities belonging to each class. The formal expressions for the TEI and SCR calculations are:

$$
\begin{aligned}
& T E I=\frac{C+C C+C E+S E+P}{S+S S} \\
& S C R=\frac{S+S E+S S}{C+C E+C C+P / 2}
\end{aligned}
$$


As can be seen in Eq. 2.6, the TEI is defined as the ratio of the sum of parameters expected to increase upon osteoclastic resorption (e.g., CE- and SEtypes), divided by the sum of parameters expected to decrease upon such processes (e.g., S-type). In this way we can evaluate the relationship between the structure voxels belonging to curve classes and the voxels of surface classes as an indicator of bone erosion.

In Eq. 2.7, likewise for the $S C R$ calculation, the numerator is compound by the sum of the surface-type voxels while the sum of curve-type voxels appear in the denominator.

\subsubsection{Anisotropy}

There are a number of methods for the anisotropy analysis of a microarchitecture. The earliest and still most widely employed is the MIL [89], which uses the mean distance between material intersections (bone-marrow interfaces) along linear traverses over a range of orientations. Because MIL traverses cross both materials, the result is a combined measure that incorporates features of each [90].

Perhaps an even more promising approach for quantifying trabecular architecture that is able to provide information on scale (e.g., thickness of structural elements), topology (plate- vs. rod-like architecture), and orientation (structural anisotropy), is tensor scale [72,91]. The local scale using a tensor model which consists in an ellipse in 2D or an ellipsoid in 3D provides a parametric representation of size, orientation, and anisotropy of local structures and is thus well suited to characterize cancellous bone networks. Regional structure is represented by a local best-fit ellipse (ellipsoid in 3D), and the structural orientation is determined from the eigenvectors along the semiaxes. The method was found to be remarkably robust over a wide range of resolution regimes and is thus applicable to in vivo imaging of trabecular bone structure $[4]$. 
Both MIL and tensor scale techniques for anisotropy characterization were developed and implemented in the thesis, not only in $2 \mathrm{D}$ but also in their $3 \mathrm{D}$ versions. In particular, the implementation of a 3D version of the tensor scale method is an important and original contribution since there are no reported studies in the literature.

\section{$\underline{\text { MIL }}$}

The basic MIL analysis performed in 2D starts by the application of lines or probes at different orientation angles through a 2D image plane containing binarized objects. The probes are perpendicular to a main line which rotates at different angles (see figure 2.26).

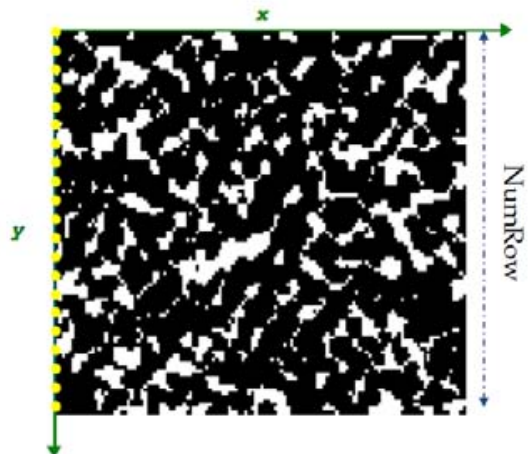

(a)

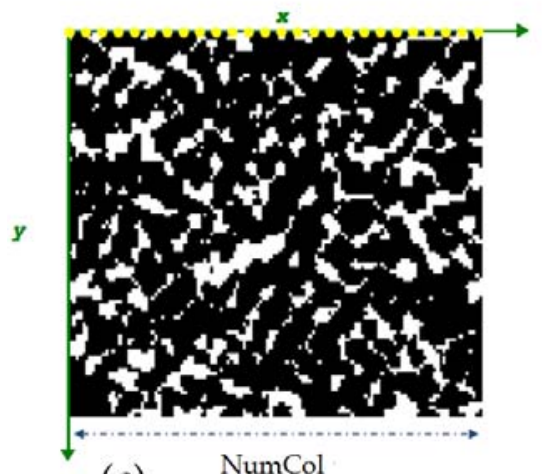

(c)

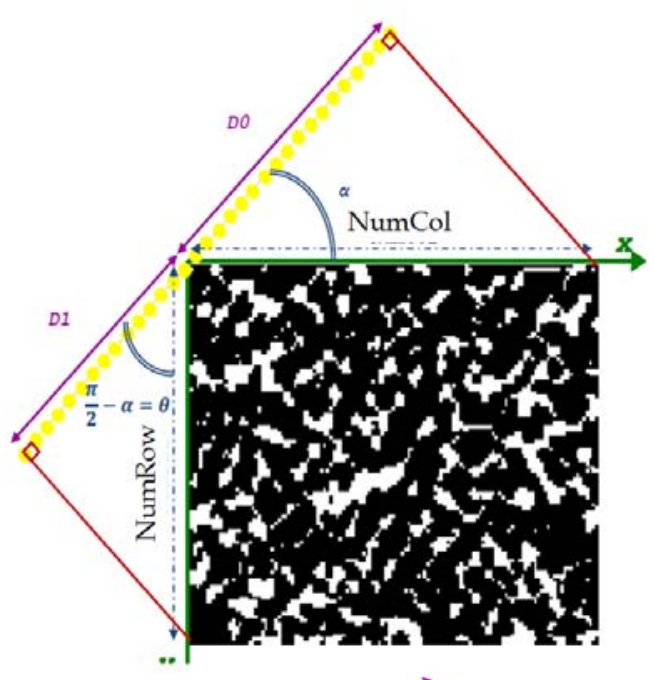

(b)

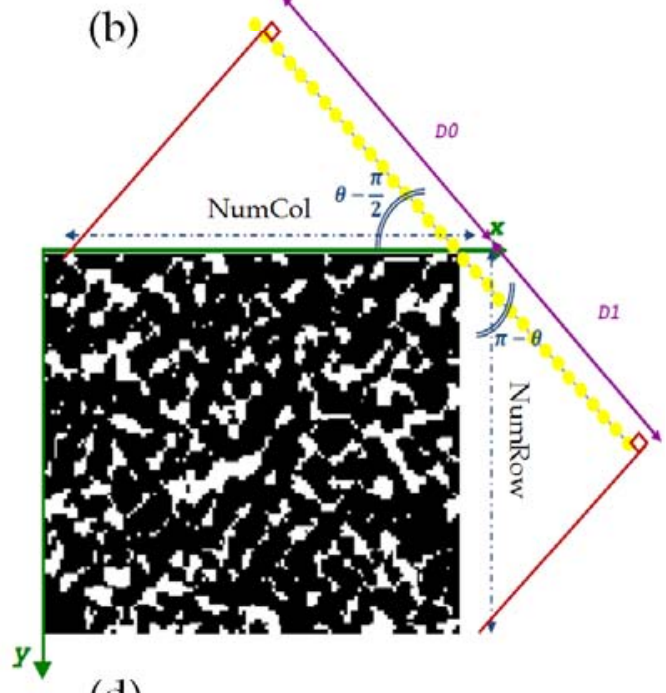

(d)

Figure 2.26. Different orientation angles of the line used for the 2D MIL calculation. Line is rotated from $\theta=0$ in (a) to $\theta=\Pi$ while sweeping the binary plane with intermediate angles (b), (c) and $(d)$ in order to analyze structure anisotropic orientation patterns. 
The length of the corresponding probe through the plane being studied is divided by the number of times that the given line passes through or intercepts part of the solid phase to obtain the MIL.

After probes rotation, a MIL value is obtained for each angle $\theta$ defined in the analysis, $\operatorname{MIL}(\theta)$. Thus, the results can be depicted in polar coordinates $\operatorname{MIL}(\theta)$, $\theta)$ and an ellipse can be fitted to the data using either least mean squares or principal component analysis (PCA). The minor and major axis of the ellipse (b and a, respectively) define the degree of anisotropy (DA) of the structure.

$$
D A=\frac{a}{b}
$$

The $D A$ is usually expressed as normalized to the major axis, that is, $a=1$. The main orientation angle $\left(\theta_{a}\right)$ of the fitted ellipse is also obtained.

In order to evaluate the implemented MIL method, the algorithm was applied to a pair of synthetic $2 \mathrm{D}$ images consisting of a circle and a rectangle (figure 2.27).

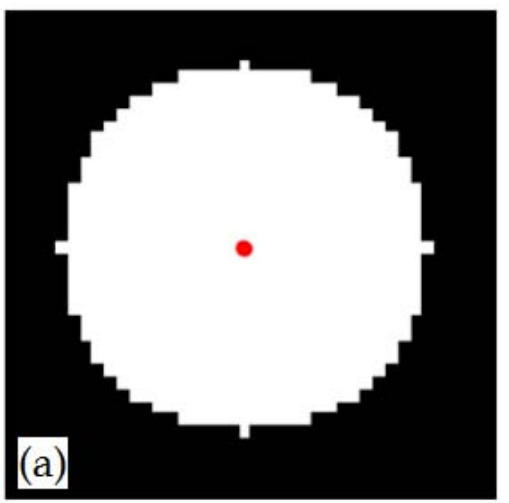

(b)
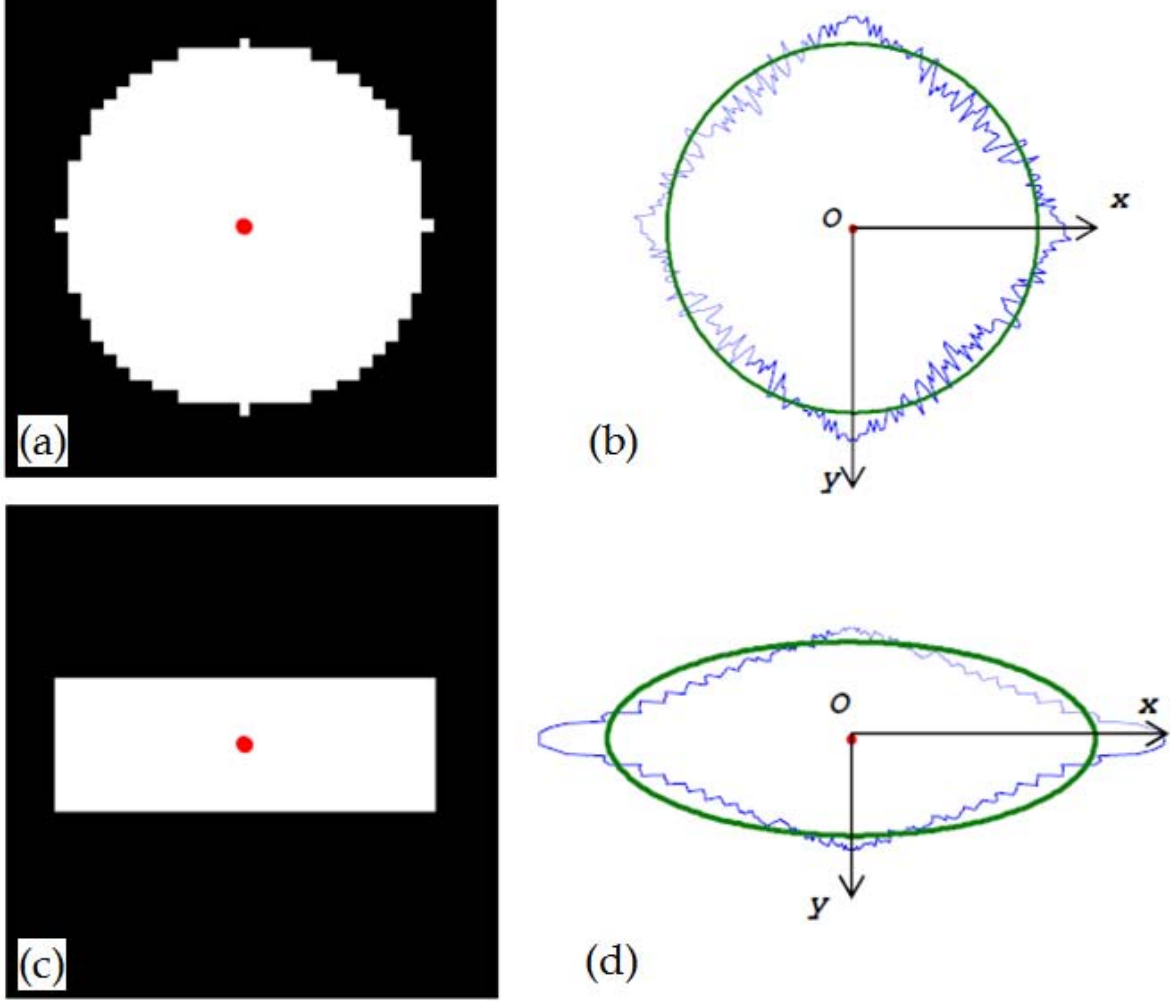

(d)

Figure 2.27. In (a), synthesized circle with its mass center in red. In (b), calculated MIL for each angle $\theta$ can be appreciated in blue, and the fitted ellipse in green. A synthesized rectangle with a clear anisotropy is observed in (c). MIL calculated for the rectangle case can be seen in blue, with the fitted ellipse in green (d). 
The extension of the algorithm for the MIL calculation to its 3D version consisted in the inclusion of the third coordinate in the geometrical equations of the probes, and also the separation of the rotation movement in two different angles, depending on the corresponding axis of reference ( $\theta$ for the $y$ axis, $\psi$ for the $z$ axis). The calculation of the MIL parameter for the given angles is analogous to the specified in the $2 \mathrm{D}$ version. In order to test the $3 \mathrm{D}$ adaptation, the algorithm was applied to a perfect sphere and to a rectangular prism, as can be observed in figure 2.28. The calculated DA for the sphere and the prism were of 1.07 and 2.12, similar to the theoretically expected (1 and 2, respectively).

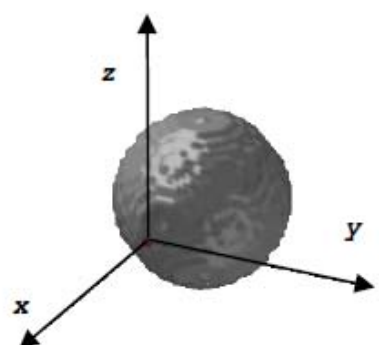

(a)

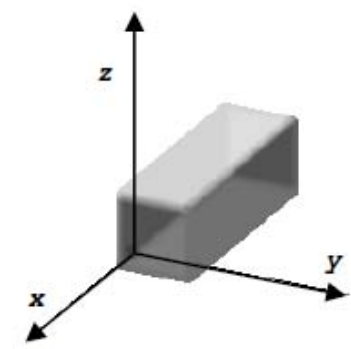

(d)

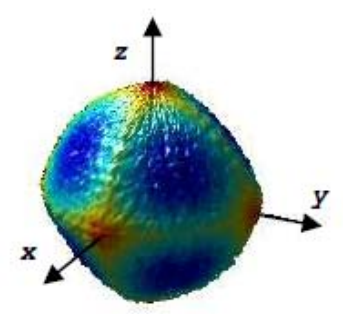

(b)

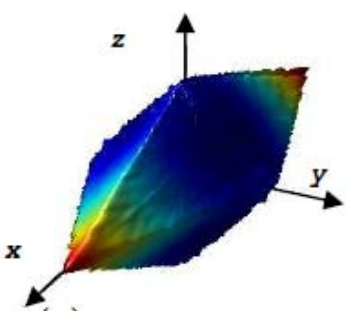

(e)

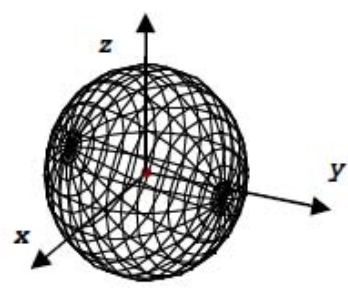

(c)

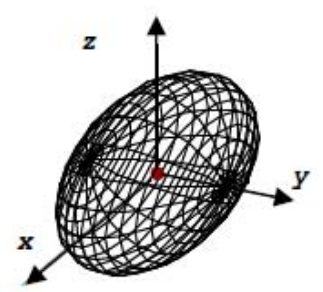

(f)

Figure 2.28. Synthesized sphere (a) and prism (d) for the evaluation of the 3D MIL algorithm. The differences in structural anisotropies between both structures can be observed in the 3D reconstruction of the MIL values measured (b) and (e). The fitted ellipsoids for the final calculation of the DA show also a clear differentiation the principal orientations.

\section{$\underline{\text { Tensor scale }}$}

The tensor scale method can be considered as a new concept of local morphometric scale using a tensor model [91]. Conceptually, the tensor scale at any image point $p$ is the parametric representation of the largest ellipse centered at $p$ that is contained in the same homogeneous region.

The method works in several sequential steps and eventually produces the tensor scale or the largest ellipse at each individual image point that is 
contained inside the local structure. For a better understanding of the purpose of each step in the detail description, a schematic illustration of the method is presented in figure 2.29. As illustrated in the figure, the method primarily considers a set of pairs of radially opposite sample lines each emanating from the candidate point and locates the closest edge on every sample line. After locating the edges on all sample lines, they are repositioned following the axial symmetry of an ellipse. Finally, the tensor scale is determined by computing the best fit ellipse to these repositioned edge points. Specifically, the entire method is completed in the following four sequential steps:

1. Intensity computation along each sample line.

2. Location of the optimum edge on each sample line.

3. Repositioning the edge locations following the axial symmetry of an ellipse.

4. PCA computation of the best fit ellipse to the repositioned edges.

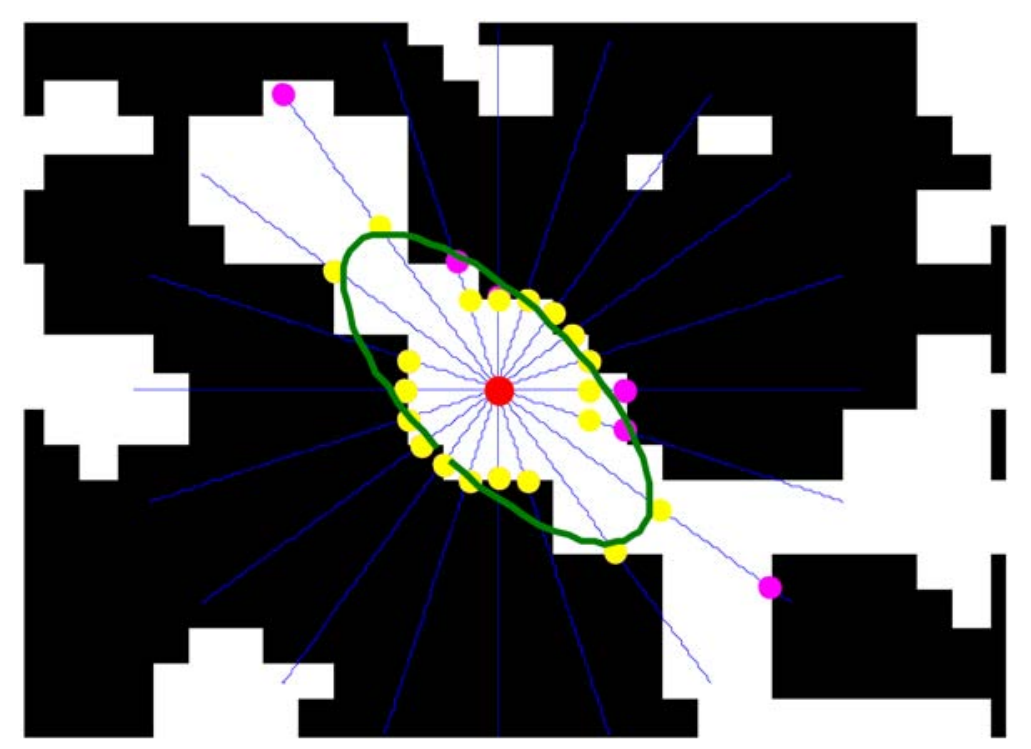

Figure 2.29. Iconography of 2D tensor scale computation at an image point (red dot). The method uses radially outward sample lines (blue) distributed at approximately a uniform angular spacing. The optimum edge (magenta dots) is located on each sample line. Following the symmetry of an ellipse, the edge points on every pair of radially opposite sample lines are repositioned (yellow dots) by selecting the one closer to the candidate point and then reflecting it on the opposite sample line. Finally, tensor scale is computed by fitting an ellipse (green) to the repositioned edge points [91].

Once the tensor scale is calculated for each point of the image, parametric maps can be built in order to graphically represent the main orientations of the 
different elements forming the image. For the parametric images showing structure anisotropy, the most usual color encoding is based on the hue saturation value (HSV) scale. In figure 2.30, an example of the application of the 2D tensor scale algorithm to a binarized fingerprint may be appreciated.

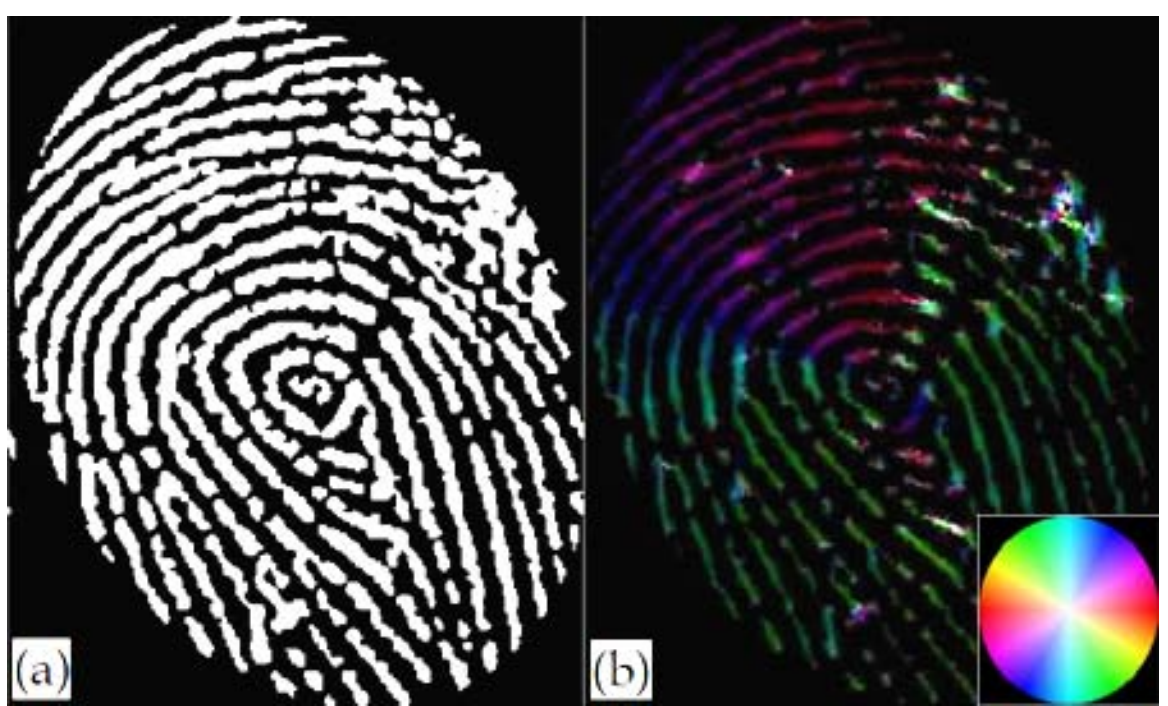

Figure 2.30. Tensor scale 2D method applied to a binarized fingerprint (a). Representation of the main orientations using HSV color coding applied to the 2D tensor scale results (b).

Regarding the extension of the tensor scale algorithm to a $3 \mathrm{D}$ version, to the doctorand knowledge, no previous reported implementations of the 3D tensor scale method were performed. Although Saha and Wehrli state that the extension of the $2 \mathrm{D}$ tensor scale algorithm to a $3 \mathrm{D}$ version is "straightforward" [72], several difficulties arise when considering the $\mathfrak{R}^{3}$ domain.

The main differences for the implementation of the 3D tensor scale arise from the ellipsoid fitting process to the calculated data and also in the representation of the results.

Analogously to 2D, the 3D tensor scale is calculated from an initial set of pairs of spatially opposite sample lines each emanating from a central voxel. The closest edges on every sample line are located. After all the edges on all sample lines have been detected, they are repositioned following the axial symmetry of an ellipsoid. An example of the application of the method to a small portion of trabecular bone in 3D can be visualized in figure 2.31 . 


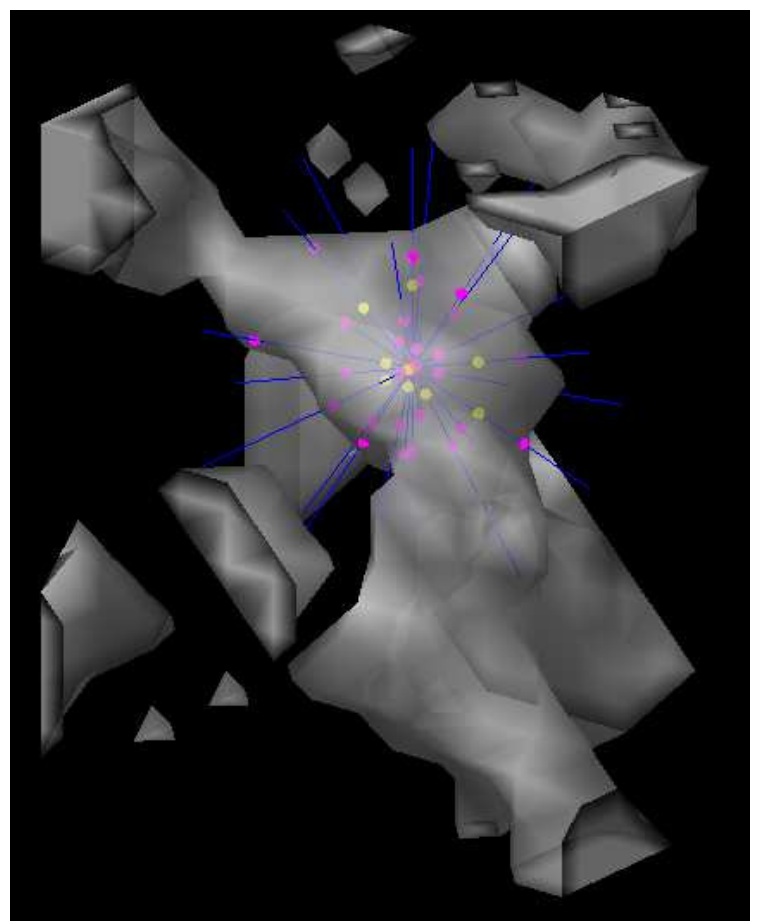

Figure 2.31. Local contour detection using a mesh of lines in the 3D tensor scale method applied to a small reconstruction of trabecular bone. (Lines: blue; Local initial boundary: magenta dots; Symmetrical local boundary: yellow dots)

The last step of the 3D version of the tensor scale method is the adjustment of an ellipsoid to the data calculated in the previous stages. In the $2 \mathrm{D}$ version, the ellipse was calculated using PCA, which was based on a statistical analysis of the position of the points to fit in order to estimate the principal direction of the ellipse. From that direction, the ellipse is rotated such that the points can be repositioned to adjust with the canonical formula of a conic section. However, when performing a rotation in $3 \mathrm{D}$, the rotation axis must be specified. Furthermore, this rotation can be decomposed into a series of rotations with respect to the coordinate axes. Also, although the directions of the principal semiaxes of the ellipsoid can be estimated similarly to $2 \mathrm{D}$, the number of rotations required in 3D increases significantly. Finally, the tensor scale was determined using an algorithm to fit the ellipsoid which contained implicit definitions of the possible rotations with respect to the coordinate axes [92]. In order to test the 3D adaptation of the tensor scale method, the algorithm was applied to the sphere and prism of figure 2.28, as can be observed in figure 2.32. In this case, as theoretically expected, the calculated DA for the sphere and the 
prism were of 1.01 and 2.03, similar to the theoretically expected ( 1 and 2, respectively).

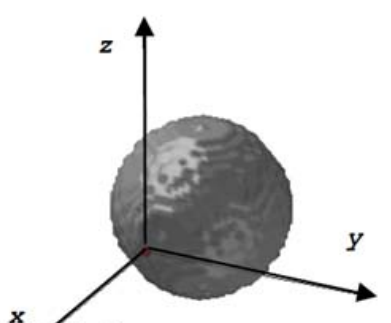

(a)

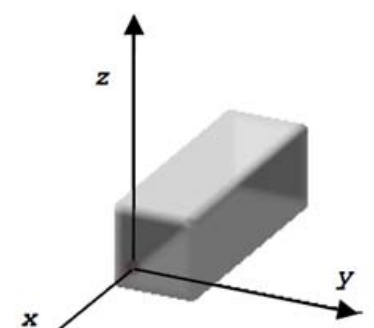

(d)

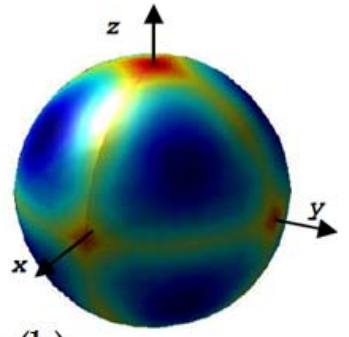

(b)

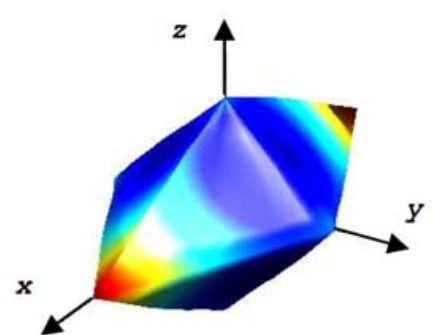

(e)

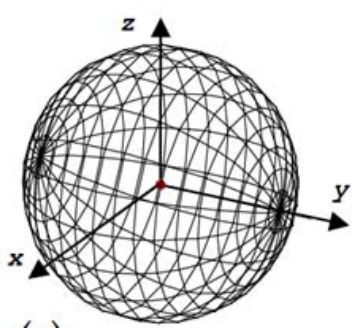

(c)

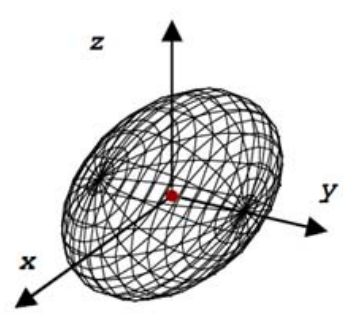

(f)

Figure 2.32. Application of the 3D tensor scale algorithm to the synthesized elements of figure 2.28 (a) and (d).

\subsection{Mechanical analysis using the FE method}

In this section, the developed methodologies for the creation of a FE model from the binarized $3 \mathrm{D}$ reconstruction obtained after image preparation are initially explained with great detail. This part continues with the particulars of the mechanical FE simulation process and the calculation of the results. Finally, the postprocessing of these results with aim of obtaining added information which is related to the bone mechanical response is also detailed.

\subsubsection{Generation of a voxel-based FE model}

As previously mentioned, at the end of the image processing chain, the trabecular bone data is in the form of a $3 \mathrm{D}$ binary matrix containing only bone and marrow voxels. However, in order to perform a FE simulation of a given 
structure, the geometry of the architecture must be fully defined in terms of small elements and their nodal connectivity. Furthermore, nodal data must be specified by the spatial localization of each node, that is, its corresponding spatial coordinates. Both elements and nodes define the mesh of the structure to be analyzed.

The most common approach to generate a FE mesh of a given geometry is using a dedicated software tool for structure meshing. Most of commercial meshing software allow for the importation of geometries information in different formats like STL or IGES (Initial Graphics Exchange Specification). These tools can efficiently generate large meshes of common engineering structures. However, in the particular case of cancellous bone modeling, in order to build reliable meshes, elements of considerably reduced dimensions (in the order of microns) are required.

Apart from the size of the elements, the type of element used for the FE mesh generation is also of key importance. The shape of the element and the number of nodes it contains define de fidelity of the mesh with the original geometry and also the number of equations to be solved per element in the global FE simulation. Several types of elements can be used to build the volumetric FE meshes. However, the most extended classes for the composition of volumetric FE models are the 4-noded tetrahedron and the 8-noded hexahedron or 'brick' element (see figure 2.33). Both sort of elements present advantages and disadvantages when used for modeling structures with very small details like the bone trabeculae. Tetrahedral elements have a better fitting to the surface geometry of the structure than hexahedrons and a smoother surface is generated with their use, thus providing a highly accurate calculation of the bone tissue loading at the trabecular surface [93]. However, the numerical requirements for the generation and solving of the FE models with this method are much larger than those for the hexahedron based models [94] and are not feasible for the characterization of bone mechanical properties in the clinical routine using conventional computation capabilities. Even more, the hexahedron model has shown excellent results when compared to the 
tetrahedron [93]. In fact, the FE meshes based on hexahedrons suppose a significantly lower computational burden in comparison to tetrahedron based meshes, and can be perfectly integrated in a clinical postprocessing platform for trabecular bone characterization. Thus, the isotropic hexahedron element was chosen in the research carried in the thesis to minimize the computational burden of posterior simulations.

\section{Tetrahedron}

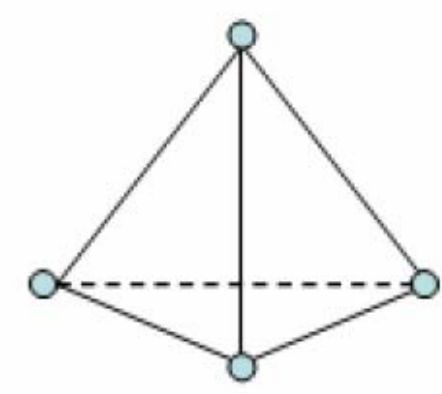

4-noded

\section{Hexahedron - Brick}

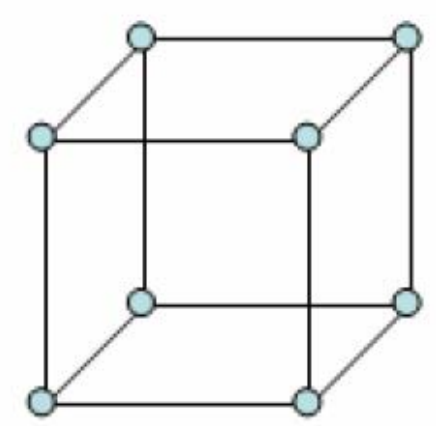

8-noded

Figure 2.33. Most common solid elements used in FE volumetric meshing.

In order to convert the $3 \mathrm{D}$ binarized reconstruction to a $\mu \mathrm{FE}$ mesh of the cancellous bone structure, a detailed description of the position of each node in cartesian coordinates must be done. This spatial localization must be combined with a complete description of the nodes of each element. An example of nodes and elements definition in a simple mesh may be appreciated in figure 2.34.

The basic idea to generate a mesh from a binarized reconstruction is to place a brick element in the structure where there is a bone voxel in the $3 \mathrm{D}$ reconstruction. This process is not trivial because of the node-sharing between different elements and the elevated size of node and element matrices which supposes high computation costs in complex structures such as trabecular bone. 


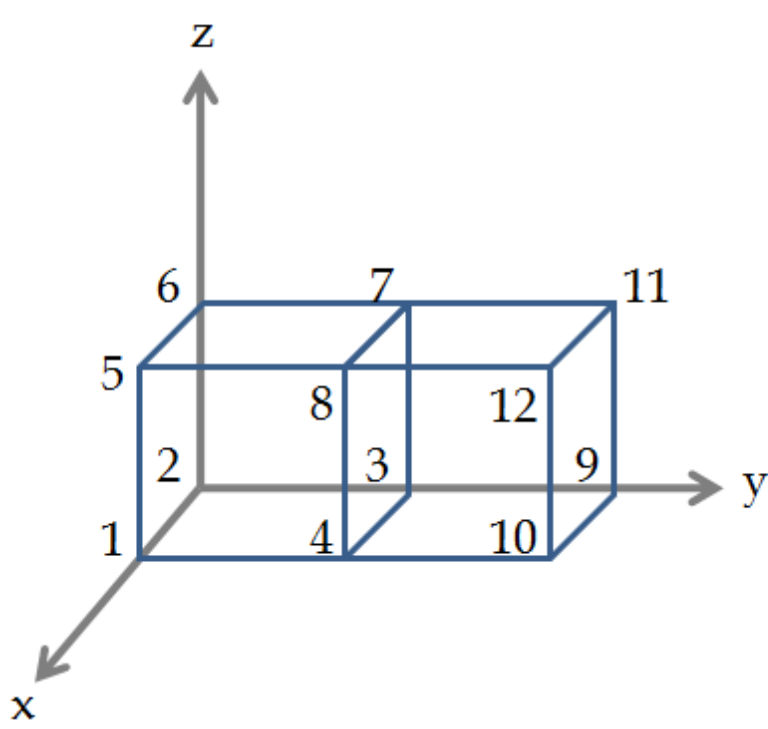

$$
\begin{aligned}
& \text { \#NODE, X, Y, Z } \\
& 1,1,0,0 \\
& 2,0,0,0 \\
& 3,0,1,0 \\
& 4,1,1,0 \\
& 5,1,0,1 \\
& 6,0,0,1 \\
& 7 \quad, 0,1,1 \\
& 8,1,1,1 \\
& 9,0,2,0 \\
& 10,1,2,0 \\
& 11,0,2,1 \\
& 12,1,2,1 \\
& \text { \# ELEMENT, \#NODE1, \#NODE2, .. } \\
& \begin{array}{l}
1 \quad, \quad 1,2,3,4,5,6,7,8 \\
2 \quad, \quad 4,3,9,10,8,7,11,12
\end{array}
\end{aligned}
$$

Figure 2.34. Example of mesh definition in a structure compound by two hexahedron elements. A list of the nodes with the specified coordinates and other list with elements connectivity must be specified.

Two different algorithms were developed to extract node and element information from a 3D reconstruction. The first one is didactic and sequential, although it has been misestimated when working with large matrices because of the high computation time. The second algorithm works directly with the whole $3 \mathrm{D}$ reconstruction matrix and can process a large number of nodes in a few seconds.

\section{Didactic algorithm}

It consists in crossing the 3D reconstruction matrix slice by slice detecting ' 1 ' elements and calculating coordinates of the corresponding nodes at each element. The main drawback is that a node can be labeled with different numbers when detecting nodes of an adjacent element. Ambiguous nodes with other node number but equal coordinates can be obtained in the node matrix. The task of looking for redundant nodes in the node matrix has a high computational cost and makes this algorithm nonviable for large matrices such as big trabecular bone reconstructions. 
2. Fast algorithm

The idea behind this algorithm is a direct detection of nodes of the structure working completely with matrices and without sequentiality [95]. Considering the $3 \mathrm{D}$ binarized reconstruction matrix of size $\mathrm{m} \times \mathrm{n} \times \mathrm{p}$, a new nodal equivalent matrix of size $(m+1) \times(n+1) \times(p+1)$ is created and filled with zeros. Then, for each bone voxel found in the $3 \mathrm{D}$ binarized reconstruction, eight ones representing the nodes of the element are situated on the corresponding position of the node equivalent reconstruction (see example on figure 2.35). The extraction of the node coordinates from the node equivalent matrix is direct. The relationship between the original bone voxel and the corresponding calculated nodes is also conserved.

\section{D binarized reconstruction}

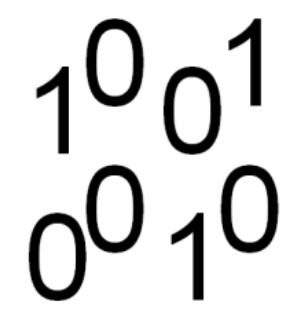

\section{Node - equivalent reconstruction}

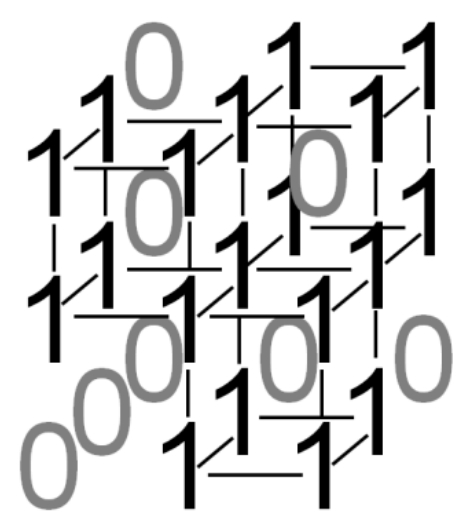

Figure 2.35. Example showing the algorithm basis for conversion from the $3 \mathrm{D}$ binarized matrix into a 3D matrix containing the node information of the structure. Node coordinates and element connectivity extraction from this process is straightforward [95].

The developed meshing algorithm can build a mesh with 1048706 nodes and 2 85148 elements in 2.78 seconds using an Intel ${ }^{\circledR}$ Core $^{\mathrm{TM}} 2$ Quad CPU at 2.83 $\mathrm{GHz}$ and $8 \mathrm{~Gb}$ of RAM memory. At the end of the process, the lists containing the nodes with the corresponding coordinates and the elements with the 
corresponding node connectivity are completed and the FE mesh is fully defined.

Once the FE mesh is fully defined in terms of nodes and elements, the bulk material properties for each element must be defined. In this case, elements' composition is supposed to be formed by compact bone, with linear, elastic and isotropic behavior [96]. Numerically, the elastic properties of the material forming the elements consist of a Young's modulus given by $E_{b}=10 \mathrm{GPa}$ and a Poisson's ratio of $v=0.3[97,98]$.

Finally, an automated software routine for saving all the model data required in simulation was implemented. The commercial platform used for mechanical simulations is ANSYS (Ansys Inc., Southpointe, PA, USA) and the input file required for simulation which contained all the data of the model was built in ANSYS format (*.ans).

A detail of the brick elements forming the FE model imported into ANSYS can be visualized in figure 2.36 .

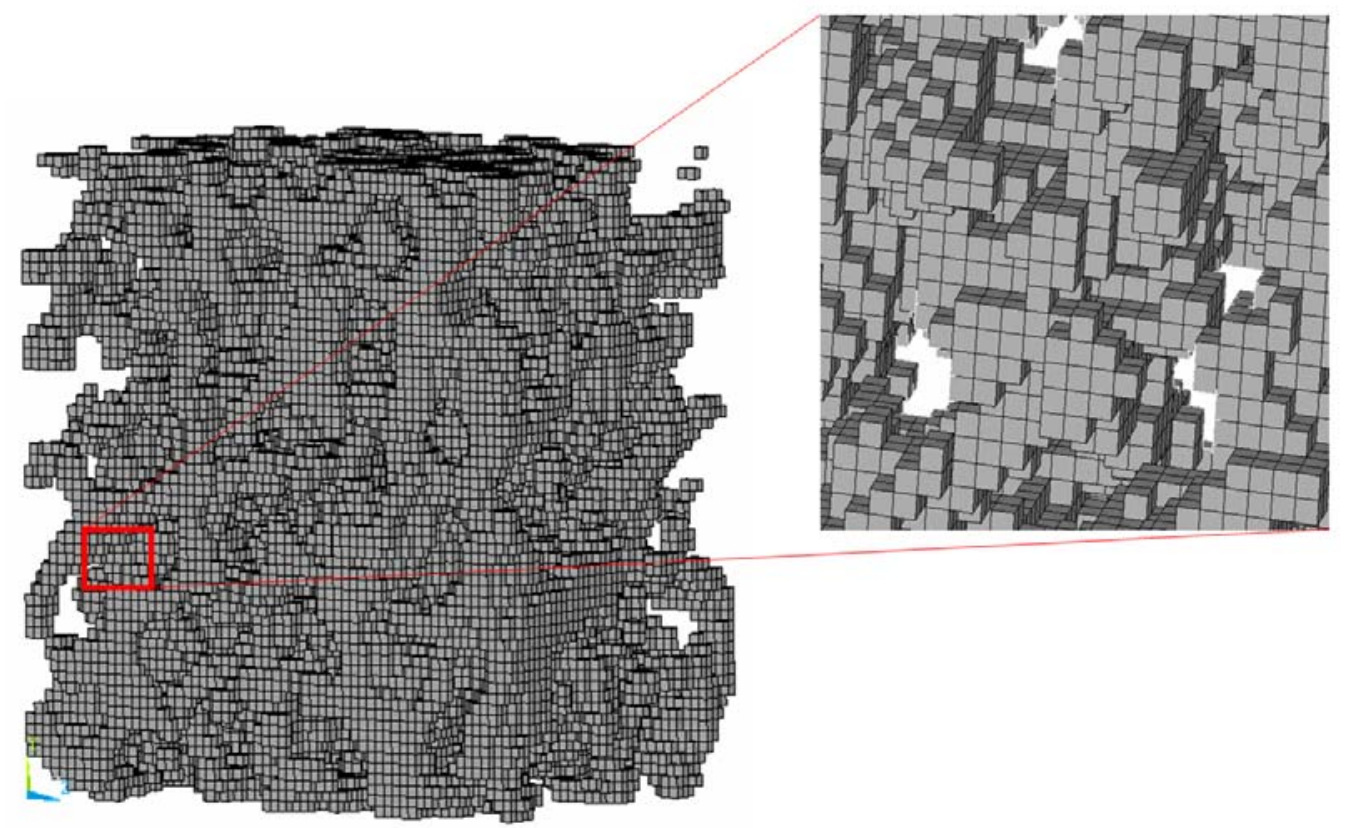

Figure 2.36. FE mesh of a trabecular bone reconstruction based on hexahedron or brick elements. Zoomed region shows a detail of the brick elements piled up compounding the structure. 


\subsubsection{Mechanical simulation of compressive conditions}

Experimentally, resistance and elasticity properties of materials and other structures are evaluated by a compression essay using specific equipment in laboratory. Stress-strain relationship is analyzed and Young's modulus of the whole structure can be easily calculated from the linear slope of the stressstrain curve.

In an analogous way, for the FE simulation, a null displacement is imposed on nodes from one side while a total strain $(\varepsilon)$ of $10 \%$ of the edge length is specified on nodes from the opposite side for the compression simulation. An iconography of the scenario to be modeled can be appreciated in figure 2.37. Although there are some results indicating that the main loading direction in the radius corresponds to the longitudinal axis [24], in order to indirectly evaluate trabecular bone anisotropy from mechanical data, the compression essay was performed uni-axially in each one of the three directions of space.

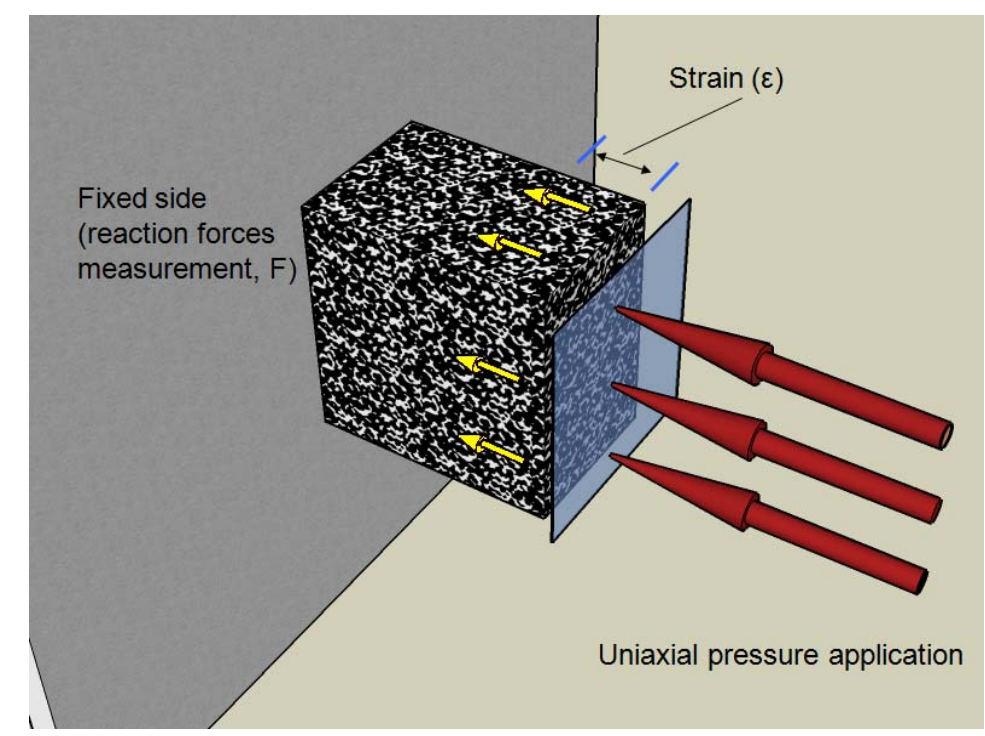

Figure 2.37. Representation of the simulation applied using the FE method. A compression test is applied to the trabecular bone sample with a side fixed to a null displacement and the opposite side suffering an imposed strain.

The modeling of a trabecular bone compression essay requires deciding the loading direction. In this case, the compressive response of the trabecular bone structure is evaluated independently in each one of the three main axis. In this 
sense, an important contribution of the thesis is based on the demonstration of the $3 \mathrm{~T}$ MR scanners advantages in terms of spatial resolution, in comparison with $1.5 \mathrm{~T}$ [66], where the slice thickness needs to have increased dimensions to maintain the SNR. Such advantages in terms of 3D spatial resolution are determinant to choose $3 \mathrm{~T}$ scanners as recommended for 3D trabecular bone characterization.

In figure 2.38a, a FE mesh of a trabecular bone structure loaded to the commercial package ANSYS 10.0 is shown. The boundary conditions and the strain application can be also appreciated in figure2.38b.

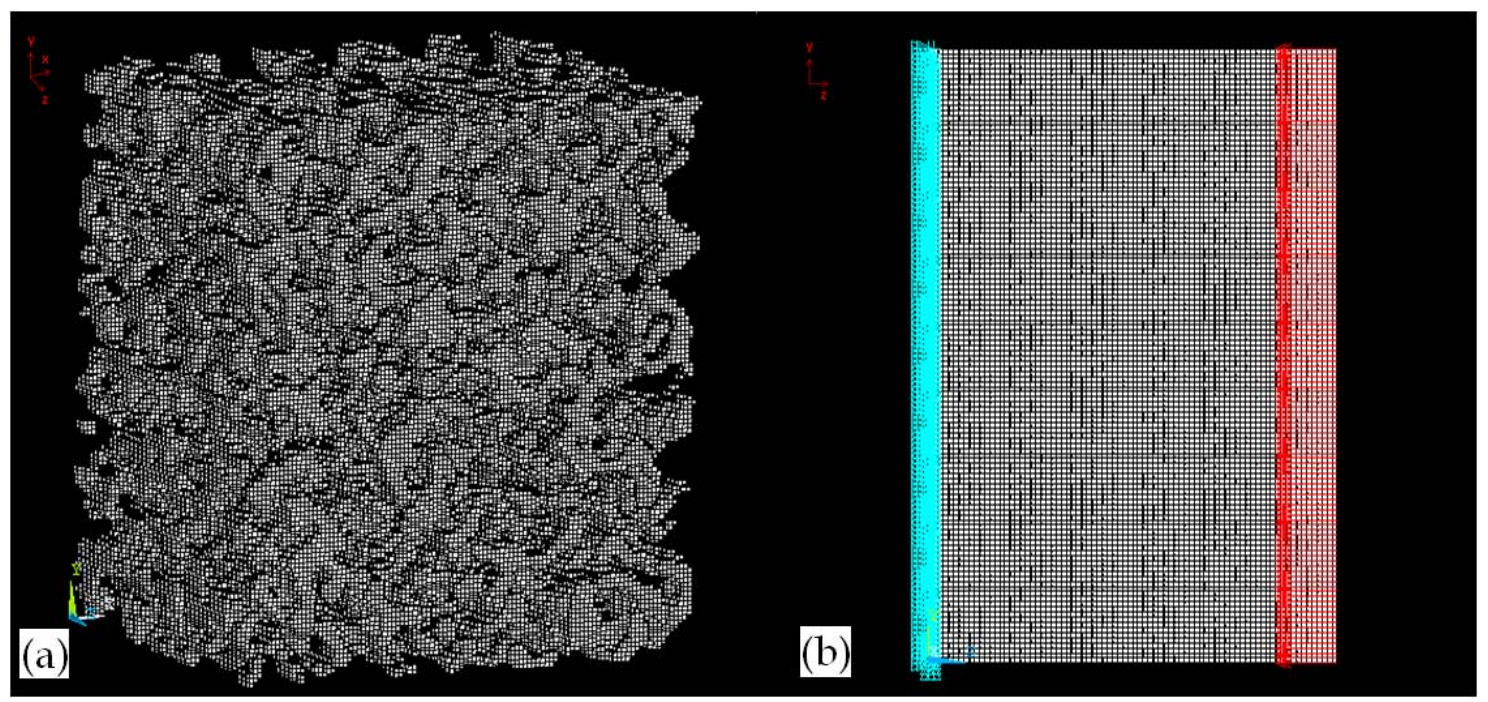

Figure 2.38. In (a), a FE mesh of trabecular bone imported in the commercial software ANSYS 10.0 is shown. The coordinates system is also shown, with the $\mathrm{z}$ direction corresponding to the longitudinal direction of the radius bone. In $b$ ), lateral view of the cancellous bone structure with boundary conditions definition, that is, null displacement on the left side (blue) and $10 \%$ strain in the right side (red).

Once the model is completely defined, including active forces application and boundary conditions, simulation process is ready to begin.

The calculation of the mechanical results from the simulation is a very demanding task in terms of computational cost. The number of equations of our system is considerably high, since there are 3 degrees of freedom per node and each element is formed by 8 nodes. As an example, a mesh with 800000 nodes defines a system of 2400000 equations. However, this number is really smaller since some nodes belong to more than one element in the same structure. 
In order to solve the large systems of equations, different strategies can be used, depending on the kind of solver (sparse, conjugate gradients, minimal residual). Computational burden can also be minimized by reduction of the dimensions of the sample under analysis. The dimensions of the complete segmented samples of trabecular bone were of $1.5 \times 1.5 \times 1 \mathrm{~cm}$ and the corresponding reduced versions were of $0.5 \times 0.5 \times 1 \mathrm{~cm}$. In the present case, for the characterization of the trabecular bone response, systems are solved using a standard sparse solver.

Although the different techniques for handling the matrices and approaching to the final solution, the mathematical problem to be solved is summarized as follows [99]:

The stiffness matrix of each element $K^{e}$ can be calculated by equation 2.9,

$$
K^{e}=\int_{V^{e}} B^{T} D B \cdot d(v o l)
$$

where $D$ is the elasticity matrix, which exclusively depends on Young's modulus and Poisson's ratio (see equation 2.10) and $B$ is a matrix exclusively containing spatial information.

$$
D=\frac{E}{1-v^{2}}\left[\begin{array}{ccc}
1 & v & 0 \\
v & 1 & 0 \\
0 & 0 & (1-v) / 2
\end{array}\right]
$$

The global stiffness matrix of the structure under analysis can be assembled by equation 2.11, taking into consideration the stiffness matrix of each element.

$$
K_{i j}=\sum K_{i j}^{e}
$$


Once the stiffness matrix is assembled, the objective of the FE problem is to solve the structural equation 2.12, which relates the global stiffness matrix with the nodal displacements $(u)$ and forces $(f)$ :

$$
K \cdot u=f
$$

After nodal displacements and forces are computed, the calculation of stresses and strains is straightforward.

\subsubsection{Processing of the results}

A very interesting parameter that can be extracted from the FE results is the apparent Young's modulus of the whole structure by the application of the homogenization theory [100, 101].

In figure 2.39, a representation of the basis of the homogeneization theory is given.

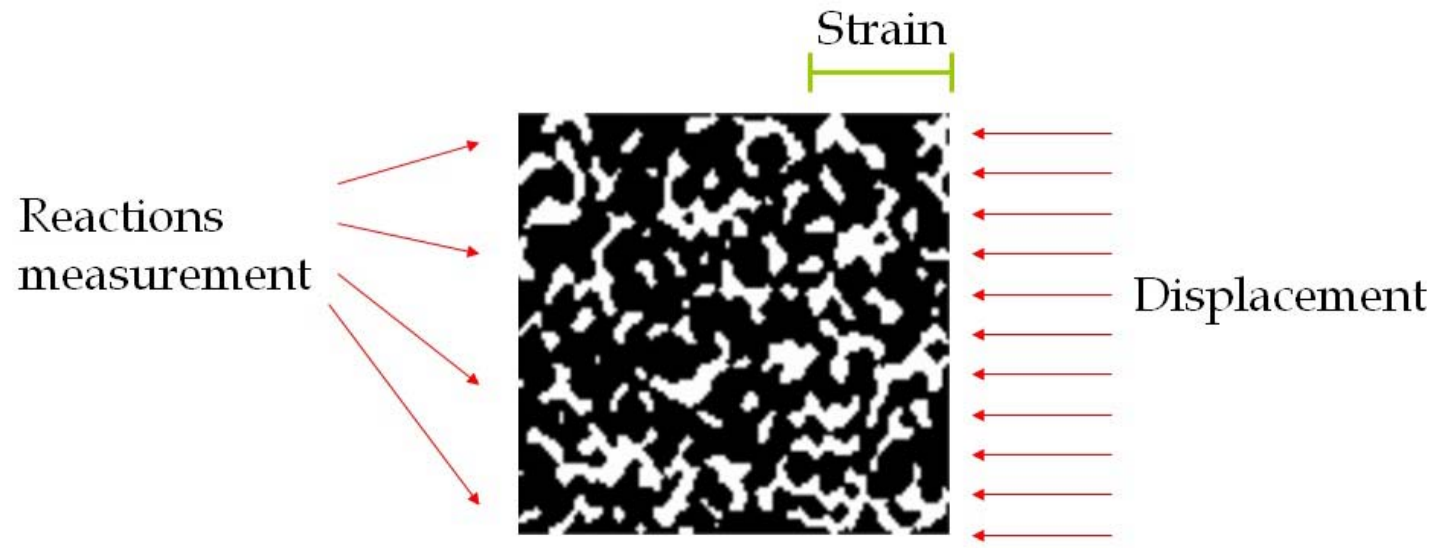

Figure 2.39. Application of the homogenization theory to the trabecular bone sample under analysis, where the whole sample is analyzed as a uniform material. Reaction forces in compression are directly measured in the opposite side.

After the application of the homogenization theory, the apparent Young's modulus of the trabecular bone tissue can be easily calculated from equation 2.13 for each space direction.

A schematic summary of the measurements computed is shown in figure 2.40: 


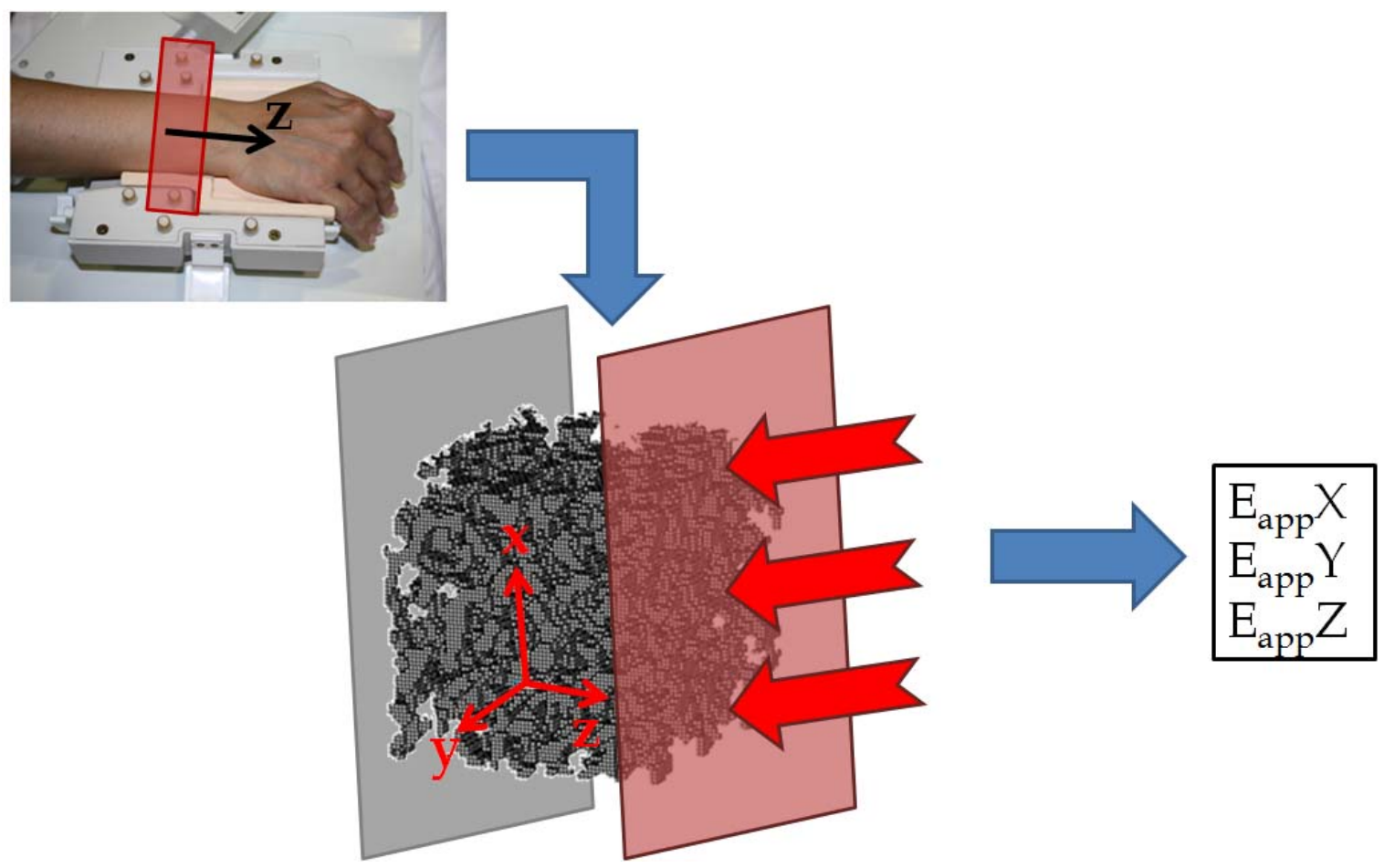

Figure 2.40. Iconography summarizing the process for trabecular bone mechanical evaluation by the application of a uniaxial compressive FE simulation. The compression is applied in the $z$ axis to calculate the $E_{a p p} Z$. The application in the $x$ and $y$ axis permits the calculation of $E_{a p p} X$ and $E_{\text {app }} Y$, respectively.

$$
E_{\text {app }}=\frac{1}{\varepsilon A} \sum_{n} F
$$

where $F$ corresponds to the nodal reaction forces measured in the fixed side, $A$ is the area of the fixed side, and $\varepsilon$ is the imposed deformation.

Apparent Young's modulus is used as an estimation of cancellous bone resistance to compression in the linear regime.

\subsection{Reproducibility and validation of the measurements}

An evaluation of the reproducibility of the developed methodology and the quality of the trabecular bone measurements was finally performed in order to 
reinforce the reliability of the cancellous bone characterization. These analyses may support the applicability of the methods, and the consideration of the estimated parameters as bone disease biomarkers.

A total of 5 fresh sheep legs were extracted from 5 adult sheep cadavers. The samples were used both for the assessment of the reproducibility and validation of the measurements.

The reason for the use of sheep tibiae and not other animal species was concluded from literature review, adult sheep offer the advantage of being of a more similar body weight to humans and having long bones of dimensions suitable for the implantation of human implants and prostheses. However, most of the published studies report that the dog is more suitable as a model for human bone from a biological viewpoint than the sheep [102, 103]. Furthermore, while the use of dogs for bone research still outnumbers sheep, over the last decade experiments with sheeps are increasing. In the last years, sheep were used in $9-12 \%$ of the orthopaedic research involving fractures, osteoporosis, bone-lengthening and osteoarthritis, in comparison with just over $5 \%$ in the eighties [104]. This increase in usage may be related to the ethical issues and negative public perception of using companion animals for medical research.

\section{Reproducibility}

For the analysis of the reproducibility of the measurements, the 5 sheep legs were scanned in three consecutive days in the same conditions than the specified in section 2.1. The region of acquisition consisted in the proximal metaphysis of the tibia (figure 2.41a), since it contained an adequate distribution of trabecular bone. One of the acquired axial slices showing the trabecular bone area of the tibia metaphysis can be seen in figure $2.41 \mathrm{~b}$. 


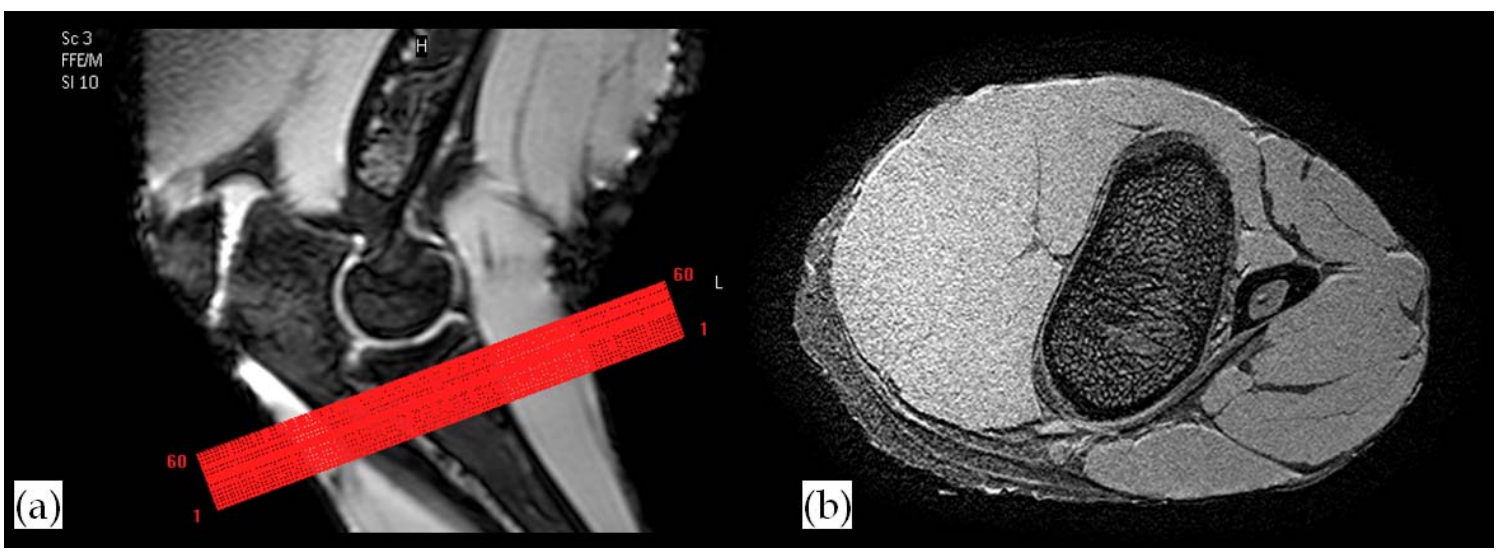

Figure 2.41. Acquisition of high spatial resolution images using the same coil and MR protocol than the specified in section 2.1. In (a), example of the planning previous to the high spatial resolution $3 \mathrm{D}$ acquisition. In (b), one of the acquired axial partitions corresponding to the region of the tibia metaphysis.

The algorithms for image processing and trabecular bone morphometry and mechanical analysis were applied to the acquisitions performed in each of the 5 sheep legs in the three different days. All the parameters were calculated for each acquisition and their reproducibility was assessed by the repeated measures analysis of variance (ANOVA) and the RMS_CoV, which can be calculated directly from [105]:

$$
R M S_{-} \operatorname{CoV}=\sqrt{\frac{\sum_{i=1}^{N}\left(\frac{\sigma_{i}}{\mu_{i}}\right)^{2}}{N} \%}
$$

where $\sigma_{i}$ is the SD, $\mu_{i}$ is the mean of the measurements and $N$ the number of cases. Low values of RMS_CoV correspond to high reproducibility in the results.

\section{Validation}

In order to perform an objective validation of the morphometry and mechanical trabecular bone parameters extracted from 3T MR images, results in the sheep tibiae were compared with high resolution $\mu \mathrm{CT}$ derived parameters in the same sheep tibiae. 
The $\mu \mathrm{CT}$ examinations required the preparation of small trabecular bone samples that were directly extracted from the tibial metaphyses using a surgery saw.

The $\mu \mathrm{CT}$ images were acquired at the facilities of the Group of Structural Mechanics and Materials Modelling (GEMM) (Aragon Institute of Engineering Research) in the University of Zaragoza, Spain. The samples were sent from Valencia to Zaragoza by urgent dedicated transport. For acquisition, a high resolution in vitro scanner (eXplore Locus SP, General Electric, USA) was used, obtaining a spatial resolution of $32 \times 32 \times 32 \mu \mathrm{m}$. The X-Ray tube voltage was of $80 \mathrm{kVp}$ and the current was of $80 \mu \mathrm{A}$.

The different morphometry and mechanical parameters were quantified in the sheep trabecular bone from $\mathrm{MR}$ and $\mu \mathrm{CT}$ acquisitions. The images resulting from the $\mu \mathrm{CT}$ examination did not need the local thresholding algorithm used in MR acquisitions for the coil shading correction. Furthermore, since the spatial resolution was considerably higher, the subvoxel processing algorithm was herein obviated.

The error in the measurements was assessed by an initial comparison of the measures using the Student's t-test and a later calculation of the relative error.

\subsection{Study groups}

The developed methodology was applied to different clinical scenarios in order to analyze the variations in the morphometry and mechanical parameters. The algorithms were applied initially to a healthy population with aim of obtaining a normality rank for the different parameters and characterize the healthy bone tissue. The alterations in the parameters under pathological conditions were analyzed in a second study in a group of post-menopausal female patients with osteoporosis. These results were compared to the obtained in a group of healthy and post-menopausal female subjects. The results for the healthy subjects in 
both groups should not be compared since the pre-processing methods were in $2 \mathrm{D}$ in the first study and in 3D in the last study of our work.

\section{Trabecular bone characterization in a healthy population}

To be considered for the study, subjects did not have to present any history of bone disease, traumatic injury to the wrist, or metabolic abnormality. All MR acquisitions were evaluated by radiologists for structures recognition and absence of artifacts. Ultimately, 40 subjects with nonsignificant wrist irregularities were selected for the study. The clinical reason for the MR examinations was to rule out articular wrist ligamentous abnormalities associated with variable degrees of pain. Because the examinations were performed as routine clinical tests, no specific informed consent was obtained for the trabecular analysis. There were 21 women and 19 men, with an age of 38 \pm 15 years (mean \pm standard deviation [SD]). Both sexes were comparable regarding age $(p=0.08$, Student's $t$ test, $34 \pm 9$ vs. $42 \pm 18$ years for men and women, respectively).

\section{$\underline{\text { Trabecular bone characterization in osteoporosis }}$}

A set of 20 female patients accomplishing World Health Organization (WHO) criteria for the diagnosis of osteoporosis (see table 1.1) [26] were included in the group of patients with osteoporosis. The mean age was of $69 \pm 7$ years (mean \pm SD). Since all the patients also underwent DXA scans with a conventional scanner (Norland XR-46, Norland Corp, Fort Atkinson, USA) due to clinical indication, the BMD measurements were available in the patients' history and were also considered in the analysis. The morphometry and mechanical results obtained for these patients were compared to those obtained in an age-matched ( $65 \pm 8$ years, $p=0.11$ ) set of 10 post-menopausal healthy females. 


\subsection{Statistical analysis}

The statistical analysis was performed in SPSS 13.0 for Windows (SPSS, Inc., Chicago, IL, USA). The limit of significance was set at $\mathrm{p}<0.05$ for all the tests. Results are expressed as mean \pm SD. Normal data distribution was checked by the application of the Kolmogorov-Smirnov test.

Comparison of the results between the healthy and osteoporotic populations was performed by the Student's $t$ test distribution for independent samples with Levene's test for the equality of variances, as data had a normal distribution.

Relationship between the different parameters was analyzed by linear regression and the Pearson's product-moment correlation coefficient in case of normal distribution. For non-normal data distribution, a Spearman's rank test was used to analyze the association of the parameters.

In the analysis where age was demonstrated to have an effect on the results of a given test, a linear general model considering the age as a co-variable was used for age correction.

The agreement between the different methods applied for the calculation of a given parameter was evaluated by the variance and was also graphically assessed by the Bland-Altman plot [106]. A Bland-Altman plot compares two assay methods. It shows the difference between the two measurements on the $Y$ axis, and the average of the two measurements on the $X$ axis.

The reproducibility analysis of the measurements was independently assessed in section 2.6 since a specific experiment was designed for that purpose. An initial repeated measures analysis of variance (ANOVA) was performed in order to evaluate the differences in the measurements. Secondly, the percentages of change of the different parameters were calculated by the root mean square coefficient of variance (RMS_CoV).

Finally, for the validation study, the relative error of the MR measurements considering $\mu \mathrm{CT}$ results as a reference was calculated. 


\section{Results and discussion}

In this chapter, the results corresponding to the application of the different developed methods to both healthy and osteoporotic populations are presented. An initial definition of normality values for all the parameters was performed in order to characterize cancellous bone under regular conditions. Secondly, the results obtained in a group of patients with osteoporosis were compared to healthy results in an age-matched population. Finally, the reproducibility and validation of our methods was assessed.

\subsection{Evaluation in a healthy population. Definition of normality ranks.}

\subsubsection{Morphometry results in healthy subjects}

In this section, the results of the application of the diverse algorithms for a morphometry characterization of the healthy spongy bone are detailed. As specified in section 2.4, the morphometry analysis consists in a quantification analysis in terms of morphology, complexity, topology and structural anisotropy indicators of the cancellous bone samples.

\section{Morphology}

The methods and algorithms detailed in section 2.4.1 for scale quantification are applied in this case.

The results of the analysis are shown in table 3.1, with the values stratified by gender. Sex has a statistically significant influence on the $B V / T V(p=0.003)$ and $\operatorname{Tb} . T h(\mathrm{p}=0.02)$ parameters, being both greater in men $(0.24 \pm 0.03$ vs. $0.21 \pm 0.04$ for $B V / T V ; 198.49 \pm 14.19 \mu \mathrm{m}$ vs. $190.35 \pm 3.95 \mu \mathrm{m}$ for $T b . T h ;$ mean \pm SD). Only a tendency on Tb.Sp ( $\mathrm{p}=0.06)$ (greater in women: $886.90 \pm 112.61 \mu \mathrm{m}$ vs. $816.52 \pm$ $115.46 \mu \mathrm{m})$ and Tb.N ( $\mathrm{p}=0.06)$ (greater in men: $1.22 \pm 0.1710^{-3} \mu \mathrm{m}^{-1}$ vs. $1.10 \pm$ 
$0.2010^{-3} \mu^{-1}$ ) was observed. These sex differences in the parameters can be graphically depicted in figure 3.1.

\begin{tabular}{|c|c|c|c|c|}
\hline $\begin{array}{c}\text { Morphological } \\
\text { parameter }\end{array}$ & $\begin{array}{c}\text { Men } \\
(\mathbf{n = 1 9 )}\end{array}$ & $\begin{array}{c}\text { Women } \\
\mathbf{( n = 2 1 )}\end{array}$ & p-value & $\begin{array}{c}\text { Total } \\
(\mathbf{n}=\mathbf{4 0})\end{array}$ \\
\hline BV/TV & $0.239 \pm 0.028$ & $0.207 \pm 0.036$ & 0.003 & $0.223 \pm 0.036$ \\
\hline Tb.Th [mm] & $0.199 \pm 0.014$ & $0.190 \pm 0.004$ & 0.02 & $0.194 \pm 0.011$ \\
\hline Tb.Sp [mm] & $0.817 \pm 0.115$ & $0.887 \pm 0.113$ & 0.06 & $0.854 \pm 0.118$ \\
\hline Tb.N [mm-1] & $1.215 \pm 0.170$ & $1.103 \pm 0.199$ & 0.06 & $1.157 \pm 0.192$ \\
\hline
\end{tabular}

Table 3.1. Mean values of morphological results for the 40 healthy subjects analyzed, expressed as the mean $\pm \mathrm{SD}$.
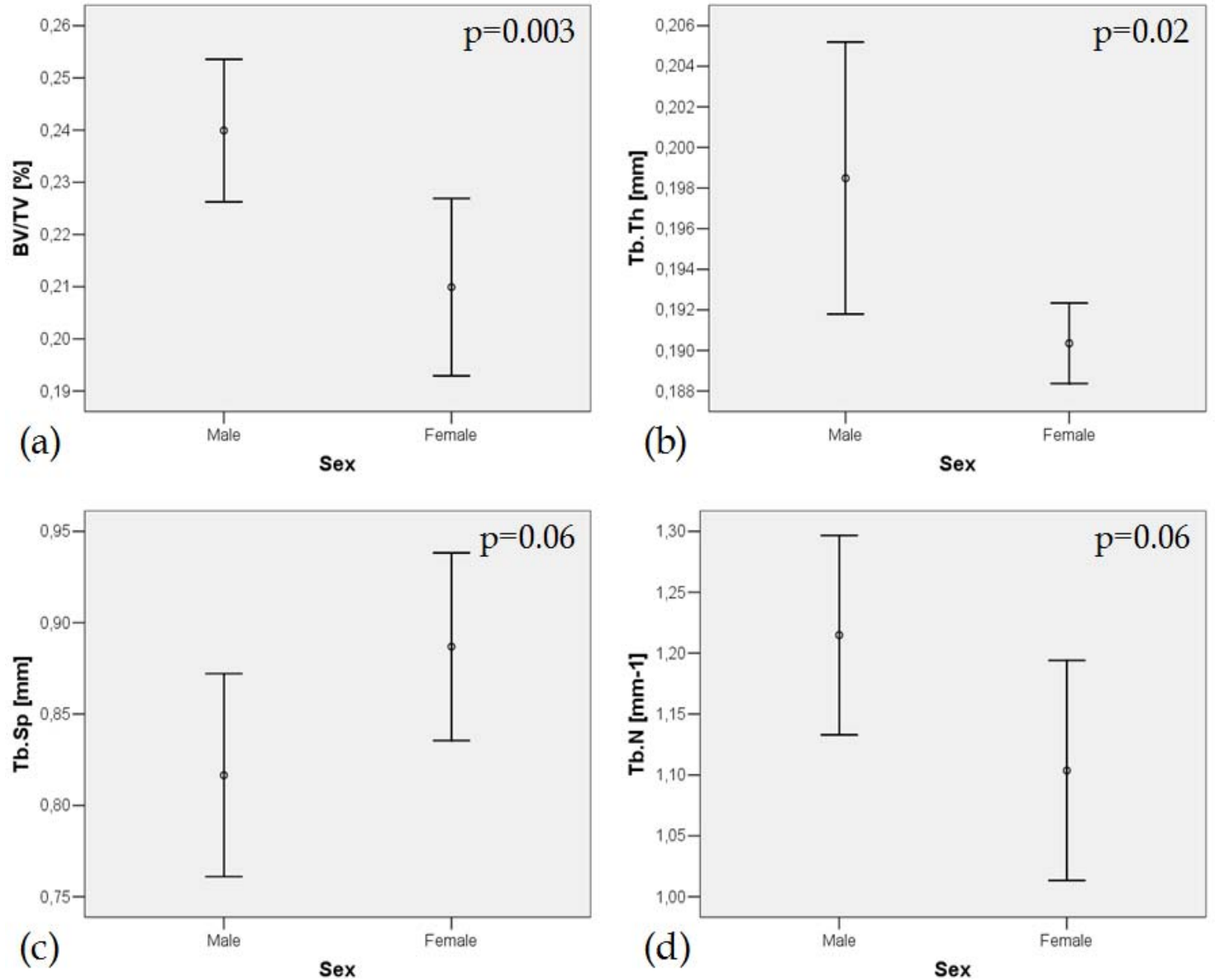

Figure 3.1. Results of the morphological quantification in the healthy population stratified by sex. Reduced overlap representing significant differences between male and female for BV/TV and Tb.Th can be observed in (a), (b), respectively. Mean results obtained for Tb.Sp and Tb.N in

(c) and (d), respectively. A higher overlap between both sexes exists in these parameters. 
Age had no statistically significant influence in any morphological parameter $(B V / T V \mathrm{r}=-0.24, \mathrm{p}=0.13$; Tb. Th $\mathrm{r}=-0.03, \mathrm{p}=0.88$; Tb.Sp $\mathrm{r}=0.12, \mathrm{p}=0.47$ and Tb.N $\mathrm{r}=-$ 0.23, $\mathrm{p}=0.16)$, neither for male nor for female healthy subjects $(B V / T V \mathrm{r}=-0.18$, $\mathrm{p}=0.46$ and $\mathrm{r}=-0.13, \mathrm{p}=0.57$; Tb.Th $\mathrm{r}=0.09, \mathrm{p}=0.72$ and $\mathrm{r}=0.20, \mathrm{p}=0.38$; Tb.Sp $\mathrm{r}=-$ 0.22, $\mathrm{p}=0.37$ and $\mathrm{r}=0.16, \mathrm{p}=0.50 ; \mathrm{Tb} . \mathrm{N} \mathrm{r}=-0.20, \mathrm{p}=0.41$ and $\mathrm{r}=-0.15, \mathrm{p}=0.52$; male and female respectively) [66].

- Discussion:

In recent years, a morphological analysis has been developed in order to test several trabecular bone physical properties, as the trabeculae dimensions and bone volume percentage $[97,98]$. In our results, gender but not age has a clear influence on the morphological bone parameters calculated from the trabecular 3D structure. Therefore, normal values of these parameters have to be stratified by gender. Variability of the obtained morphological parameters may be also influenced by the acquisition and image processing techniques [66]. In our study, the obtained morphological parameters were in accordance with those previously published in other studies $[55,98]$. However, some differences have to be mentioned. Our mean $B V / T V$ was lower compared to Newitt DC et al. $(0.22 \pm 0.04$ vs. $0.36 \pm 0.05$, respectively) [98]. As these authors studied morphological parameters results in a population of post-menopausal females (58 \pm 7 years old) and used a higher 3D slice partition thickness (500 $\mu \mathrm{m})$, these differences in spatial resolution and age distribution may be responsible for the disparity. Our mean $\mathrm{Tb}$.Th is in accordance to the one obtained in [98], (0.19 \pm $0.01 \mathrm{~mm}$ vs. $0.21 \pm 0.02 \mathrm{~mm}$ ) since the method of distance transform is characterized by its robustness on those cases having a higher spatial resolution than the typical dimensions of the tissue under study [84]. Our mean Tb.Sp is also similar to the one obtained in [55] for trabecular bone samples of the radius $(0.85 \pm 0.12 \mathrm{~mm}$ vs. $0.75 \pm 0.35 \mathrm{~mm})$. Results obtained for $T b . N$ are also close to those published in [55] $\left(1.16 \pm 0.19 \mathrm{~mm}^{-1}\right.$ vs. $\left.0.96 \pm 0.20 \mathrm{~mm}^{-1}\right)$. 


\section{Complexity}

The $2 \mathrm{D}$ and $3 \mathrm{D}$ routines for the calculation of $D^{2 D}$ and $D^{3 D}$ were applied to the population of healthy subjects. The mean value obtained for $D^{2 D}$ was of $1.71 \pm$ 0.07 , with higher values in men $(1.73 \pm 0.06$ vs. $1.69 \pm 0.06, p=0.04$; men vs. women, respectively). The mean $D^{3 D}$ value was of $2.33 \pm 0.05$, with again increased values in the case of men $(2.35 \pm 0.05$ vs. $2.32 \pm 0.05, p=0.05$; men vs. women, respectively). These results are summarized in table 3.2 and graphically assessed in figure 3.2.

\begin{tabular}{|c|c|c|c|c|}
\hline $\begin{array}{c}\text { Fractal } \\
\text { dimension }\end{array}$ & $\begin{array}{c}\text { Men } \\
(\mathbf{n = 1 9 )}\end{array}$ & $\begin{array}{c}\text { Women } \\
\mathbf{( n = 2 1 )}\end{array}$ & p-value & $\begin{array}{c}\text { Total } \\
\mathbf{( n = 4 0 )}\end{array}$ \\
\hline D $^{2 \mathrm{D}}$ & $1.73 \pm 0.06$ & $1.69 \pm 0.06$ & 0.04 & $1.71 \pm 0.07$ \\
\hline D $^{3 \mathrm{D}}$ & $2.35 \pm 0.05$ & $2.32 \pm 0.05$ & 0.05 & $2.33 \pm 0.05$ \\
\hline
\end{tabular}

Table 3.2. Mean values of fractal complexity results for the 40 healthy subjects analyzed expressed as the mean \pm SD.

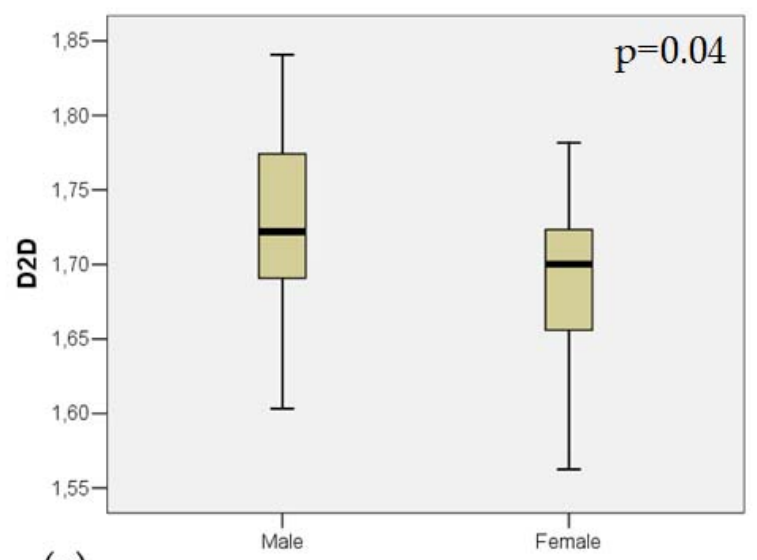

(a)

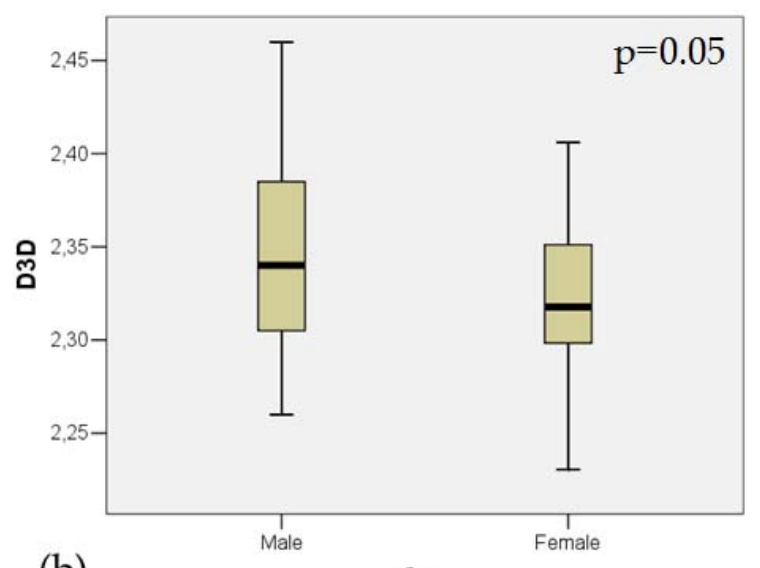

(b)

Sex

Figure 3.2. Results of the fractal dimension quantification in the healthy population stratified by sex. Although statistically significant, a high overlap between both sexes exists in these parameters. Mean results obtained for $D^{2 D}$ and $D^{3 D}$ in (a) and (b), respectively.

No significant relationships were found between $D^{2 D}, D^{3 D}$ and age $(\mathrm{r}=-0.02$, $\mathrm{p}=0.94$ and $\mathrm{r}=-0.31, \mathrm{p}=0.18 ; D^{2 D}$ vs. age, $D^{3 \mathrm{D}}$ vs. age). 
- Discussion:

Gender seems to have a higher influence on the 2D measurements than 3D. Although the differences in the results between males and females can be considered as significant, there exists a large overlap in the results distribution, as it may be appreciated in figure 3.2.

The calculation of $\mathrm{D}^{3 \mathrm{D}}$ values from high-spatial resolution MR measurements of trabecular bone was found to be feasible if applied after proper image processing and efficient contour detection algorithms. To doctorand knowledge, 3D fractal algorithms have not been previously prepared and applied to the study of trabecular bone from high spatial resolution MR acquisitions [107].

\section{$\underline{\text { Topology }}$}

The surface-to-curve ratio (SCR) and total erosion index (TEI) parameters were calculated in the healthy group in order to characterize topological properties of the normal spongy bone. As seen in section 2.4.3, voxels of the skeletonized structure are classified into 9 different groups depending on their situation in the architecture. From this classification, the SCR and TEI parameters were derived.

The results for the TEI and SCR were significantly influenced by sex. The mean value for TEI was of $12.43 \pm 4.96$, with significantly higher values in women (9.94 \pm 4.23 vs. 14.52 $\pm 4.63, \mathrm{p}=0.005$; men vs. women, respectively). As a complementary, the mean value for $S C R$ was of $0.41 \pm 0.11$, with significantly higher values in men $(0.46 \pm 0.11$ vs. $0.36 \pm 0.08, p=0.005$; men vs. women, respectively). The results obtained are summarized in the table 3.3 and commented age influences can be visualized in figure 3.3. 


\begin{tabular}{|c|c|c|c|c|}
\hline $\begin{array}{c}\text { Topology } \\
\text { indicator }\end{array}$ & $\begin{array}{c}\text { Men } \\
(\mathbf{n = 1 9 )}\end{array}$ & $\begin{array}{c}\text { Women } \\
\mathbf{( n = 2 1 )}\end{array}$ & p-value & $\begin{array}{c}\text { Total } \\
(\mathbf{n}=\mathbf{4 0})\end{array}$ \\
\hline TEI & $9.94 \pm 4.23$ & $14.52 \pm 4.63$ & 0.005 & $12.43 \pm 4.96$ \\
\hline SCR & $0.46 \pm 0.11$ & $0.36 \pm 0.08$ & 0.005 & $0.41 \pm 0.11$ \\
\hline
\end{tabular}

Table 3.3. Mean values of topology results for the 40 healthy subjects analyzed expressed as the mean \pm SD.
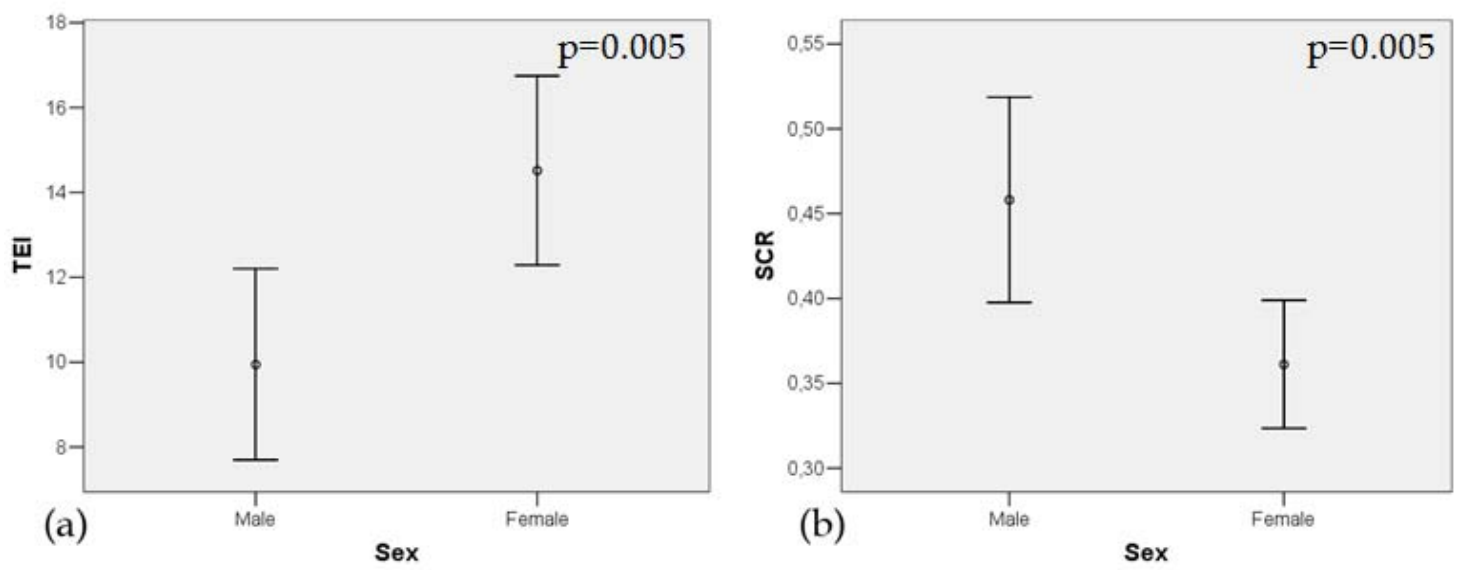

Figure 3.3. Results of the TEI and SCR parameters in the healthy population. Mean results and standard deviation obtained for TEI and SCR in (a) and (b), respectively.

No relationships were found between age and both topological parameters $(\mathrm{r}=0.17, \mathrm{p}=0.32$, age vs. TEI; $\mathrm{r}=-0.07, \mathrm{p}=0.70$, age vs. $S C R)$.

- Discussion:

The digital topological analysis (DTA) is specially designed for the early detection of bone erosion processes. The TEI and SCR topological have been proven to be useful as discriminators of different structural arrangements and to be sensitive indicators for the conversion of plates to rods [70]. Perforation of plates, for example, decreases the number of surface-interior voxels. Likewise, disruption of rods decreases the number of curve-interior (C-type) while increasing the number of curve-edge (CE-type) voxels. Pothuaud et al [108, 109] also conceived a method where the images are processed to yield a 3D skeleton line graph from which topological indices are determined. One limitation of this 
approach is that no distinction is made in the treatment of plates and rods during the skeletonization process.

In the approach exposed herein, the skeletonization algorithm applied before topological classification was specially prepared for a different treatment of plates and rods in the cancellous bone structure to be skeletonized.

\section{Anisotropy}

Both 3D MIL and 3D tensor scale methods were applied to the healthy group in order to fully characterize normal trabecular bone orientation patterns. Although two angles are needed in order to define an orientation in the $3 \mathrm{D}$ space, results are only given for the $\theta_{a}$ parameter and not $\psi_{a}$ because the first gives an idea of the main orientation in the $x-y$ plane. The results obtained using both methods showed no statistical differences for $\theta_{a}\left(71.63 \pm 13.90^{\circ}\right.$ vs. $69.57 \pm 13.85^{\circ}, p=0.53$. 3D-MIL vs. 3D-Tensor Scale). However, in the case of the degree of anisotropy $(D A)$ calculation, significant differences were obtained (1.09 \pm 0.03 vs. $1.12 \pm 0.04, p=0.05$. 3D-MIL vs. 3D-Tensor Scale).

The results for 3D-MIL and 3D-Tensor Scale methods stratified by sex can be appreciated in table 3.4. There were no significant differences between sexes for the parameters analyzed ( $\mathrm{p}=0.86$ and $\mathrm{p}=0.43$ for $3 \mathrm{D}-\mathrm{MIL} \theta_{a}$ and $D A ; \mathrm{p}=0.82$ and $\mathrm{p}=0.74$ for 3D-Tensor Scale $\theta_{a}$ and $D A$ ). However, a slight trend to an increased in $D A$ for the women was observed.

\begin{tabular}{|c|c|c|c|c|}
\hline $\begin{array}{l}\text { Anisotropy } \\
\text { parameter }\end{array}$ & $\begin{array}{c}\text { Men } \\
(n=19)\end{array}$ & $\begin{array}{l}\text { Women } \\
(n=21)\end{array}$ & p-value & $\begin{array}{c}\text { Total } \\
(n=40)\end{array}$ \\
\hline $\boldsymbol{\theta}_{\mathbf{a}}{ }^{\mathbf{o}}(3 \mathrm{D}-\mathrm{MIL})$ & $71.13 \pm 11.45^{\circ}$ & $71.94 \pm 15.46^{\circ}$ & 0.86 & $71.63 \pm 13.90^{\circ}$ \\
\hline $\begin{array}{c}\boldsymbol{\theta}_{\mathbf{a}}{ }^{\mathbf{o}}(3 \mathrm{D}-\text { Tensor } \\
\text { Scale })\end{array}$ & $62.85 \pm 14.84^{\circ}$ & $73.77 \pm 13.33^{\circ}$ & 0.82 & $69.57 \pm 13.85^{\circ}$ \\
\hline DA (3D-MIL) & $1.08 \pm 0.03$ & $1.09 \pm 0.04$ & 0.43 & $1.09 \pm 0.03$ \\
\hline $\begin{array}{c}\text { DA (3D-Tensor } \\
\text { Scale) }\end{array}$ & $1.12 \pm 0.03$ & $1.12 \pm 0.04$ & 0.74 & $1.12 \pm 0.04$ \\
\hline
\end{tabular}

Table 3.4. Mean values of anisotropy results (expressed as the mean \pm SD). using both MIL and tensor scale methods implemented in 3D. Application to the group of 40 healthy subjects. 
Since two different methods are used to measure the same parameters, data comparison can be intuitively performed by the Bland-Altman plots in figures 3.4 and 3.5, for $\theta_{a}$ and $D A$, respectively. As it can be concluded, the results obtained with the different methods applied show differences.

No significant relationships between age and any of the anisotropy parameters was observed either for 3D-MIL ( $\mathrm{r}=-0.37, \mathrm{p}=0.82$ for $\theta_{a}$ vs. Age and $\mathrm{r}=-0.09$, $\mathrm{p}=0.60$ for $D A$ vs. Age) or 3D-Tensor Scale $\left(\mathrm{r}=0.22, \mathrm{p}=0.06\right.$ for $\theta_{a}$ vs. Age and $\mathrm{r}=-$ 0.05, $\mathrm{p}=0.78$ for $D A$ vs. Age).

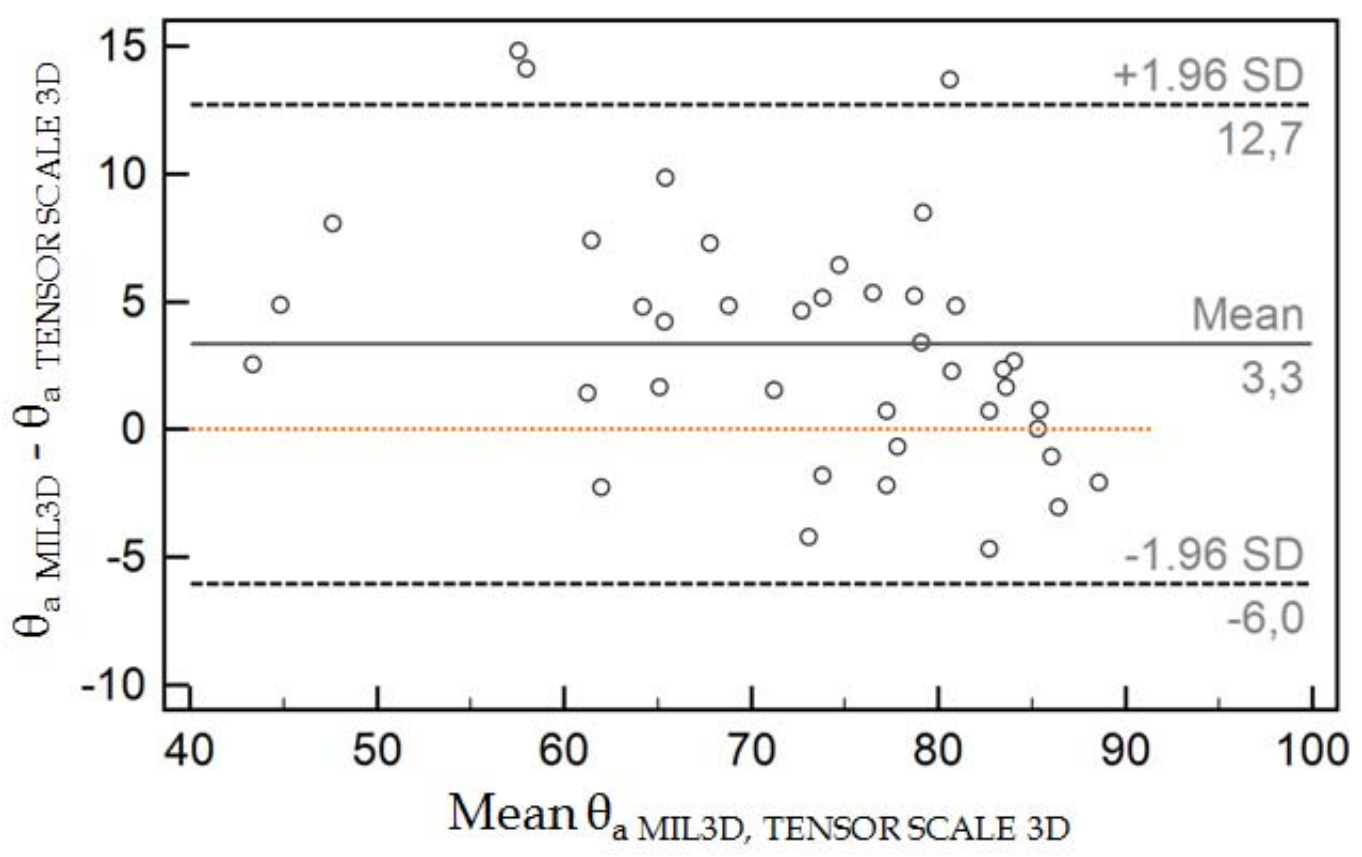

Figure 3.4. Bland-Altman plot showing $\theta_{a}$ differences distribution for both 3D-MIL and 3D-Tensor Scale methods. 


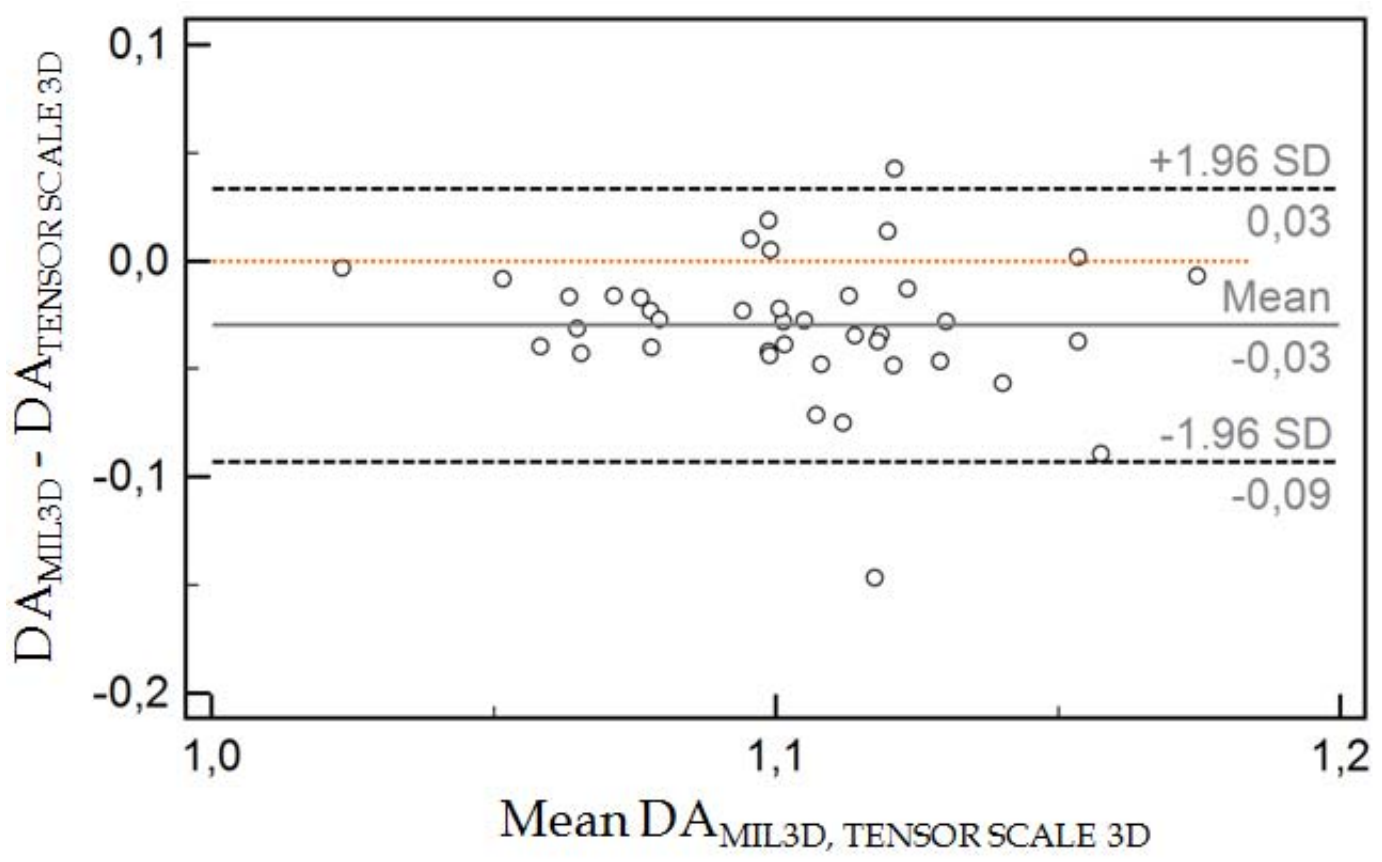

Figure 3.5. Bland-Altman plot showing $D A$ differences distribution for both 3D-MIL and 3D-Tensor Scale methods.

In figure 3.6, the graphical results of the application of 3D-MIL and 3D-Tensor Scale to a healthy trabecular bone sample can be appreciated.

(a)
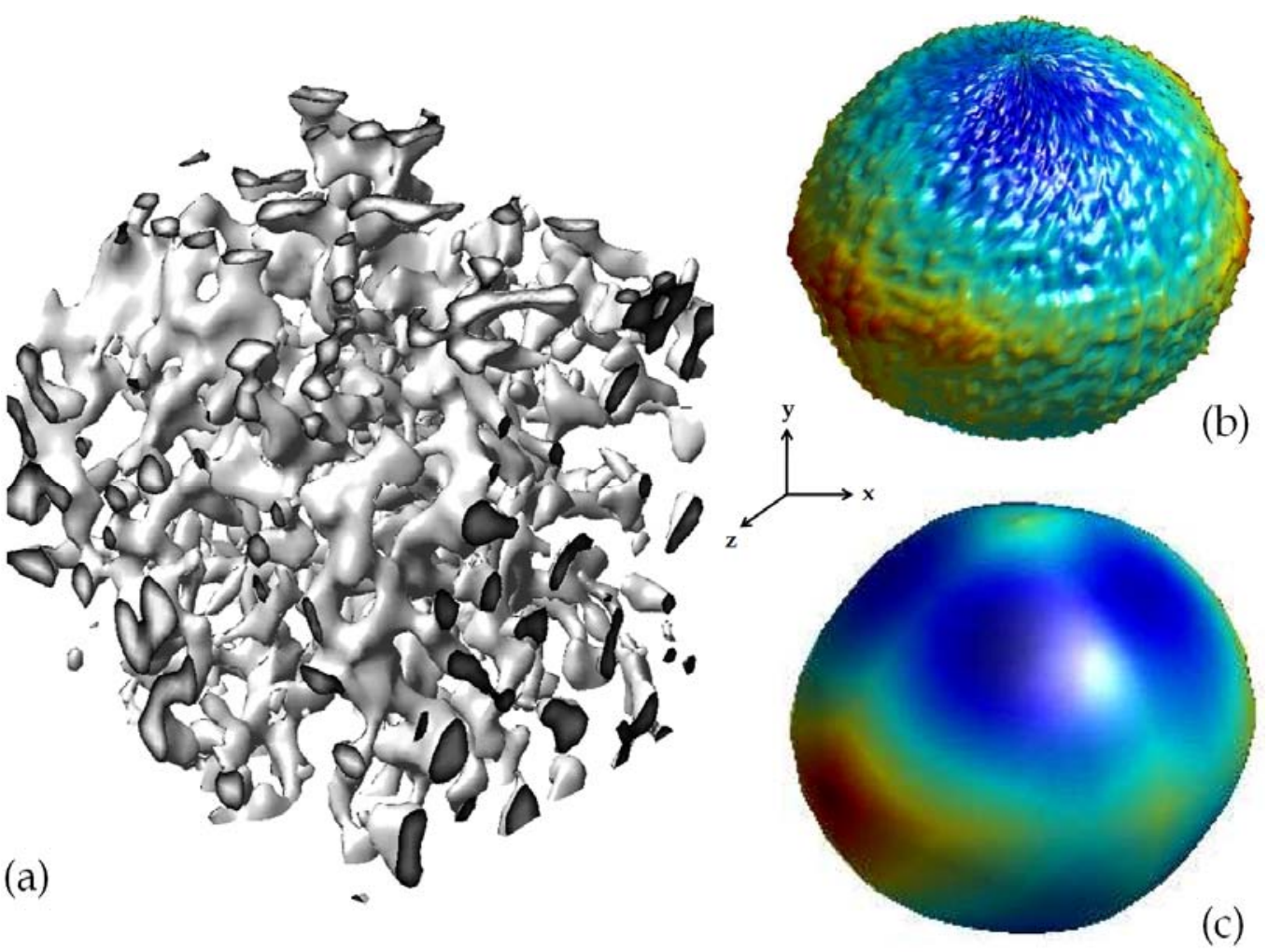

Figure 3.6. Application of the 3D-MIL and 3D-Tensor Scale methods to a healthy trabecular bone sample. In (a), 3D reconstruction of the cancellous bone network. In (b), 3D-MIL surface for the ellipsoids fitted to the structure. In (c), surface of the 3D-Tensor Scale fittings. 
- Discussion:

The application of the 3D-MIL and 3D-Tensor Scale provided information about the internal structural organization of bone rods and plates. The application of both methods to the series of healthy trabecular bone volumes confirmed that the trabeculae are mainly oriented along the $z$ axis, which corresponds to the radius longitudinal direction $[110,111]$. The example in figure 3.6 clearly shows the principal orientation of the fitted ellipsoids either for 3D-MIL (figure 3.6b) or for 3D-Tensor Scale (figure 3.6c).

Both 3D-MIL and 3D-Tensor Scale methods were applied to the anisotropy characterization of the trabecular bone with satisfactory results. However, there were significant differences in the calculation of the $D A$ parameter by these approaches, with significantly higher $D A$ values in the case of 3D-Tensor Scale. A study was performed in [92] in order to evaluate the variation of the anisotropy parameters with the selected volume of interest. Neither MIL nor Tensor Scale showed differences between 5 different VOI types, whereas the inertia ellipsoid method showed significant variations. This was the reason for not including the inertia ellipsoid as a method for anisotropy analysis in the present thesis.

In accordance with our results, the 3D-Tensor Scale method presents a higher sensitivity to structure orientation quantification [4] and also provides added information in terms of local analysis of scale and anisotropy in a voxel-byvoxel basis [112]. The 3D-Tensor Scale method is preferred for the anisotropy analysis of trabecular bone structures.

\subsubsection{Mechanical results in healthy subjects}

In this section, the results of the processes explained in section 2.5 for a mechanical characterization of the cancellous bone are presented. After the simulation of the FE models of the trabecular bone samples under compressive conditions in the three directions of space, the elastic modulus may be quantified. Parametric stress reconstructions from the FE simulations are 
initially presented and discussed. Then, the values obtained for the Young's modulus after post-processing of the FE simulation results in the healthy trabecular bone are presented. Due to the high computational cost of the FE simulations, the effect of reducing the volume of interest (VOI) (as introduced in section 2.5.2) in the sample under analysis is also exposed. Finally, the influence of the skeleton shape and the importance of its microarchitecture are analyzed by a comparison of the Young's modulus calculated from the skeletonized volumes resulting from the topology analysis process and nonskeletonized original samples.

\section{$\underline{\text { Parametric 3D reconstructions of nodal stresses }}$}

The different nodal solutions (stresses, strains, displacements, forces) from the FE simulation can be represented in parametric $3 \mathrm{D}$ reconstructions in order to graphically evaluate regions with certain mechanical conditions.

The trabecular bone of a healthy subject tends to present a homogeneous stress distribution in all the cancellous bone structure when subjected to compressive conditions. This efficient stress distribution is the consequence of a good transmission of loads between bone rods and surfaces. In figure 3.7a it can be appreciated an example of nodal stress parametric map obtained from the simulation of compression in the z-axis of a healthy trabecular bone sample. In the case of bone microarchitecture deterioration, stress tends to be localized in heterogeneous distributed regions as shown in figure $3.7 \mathrm{~b}$. 


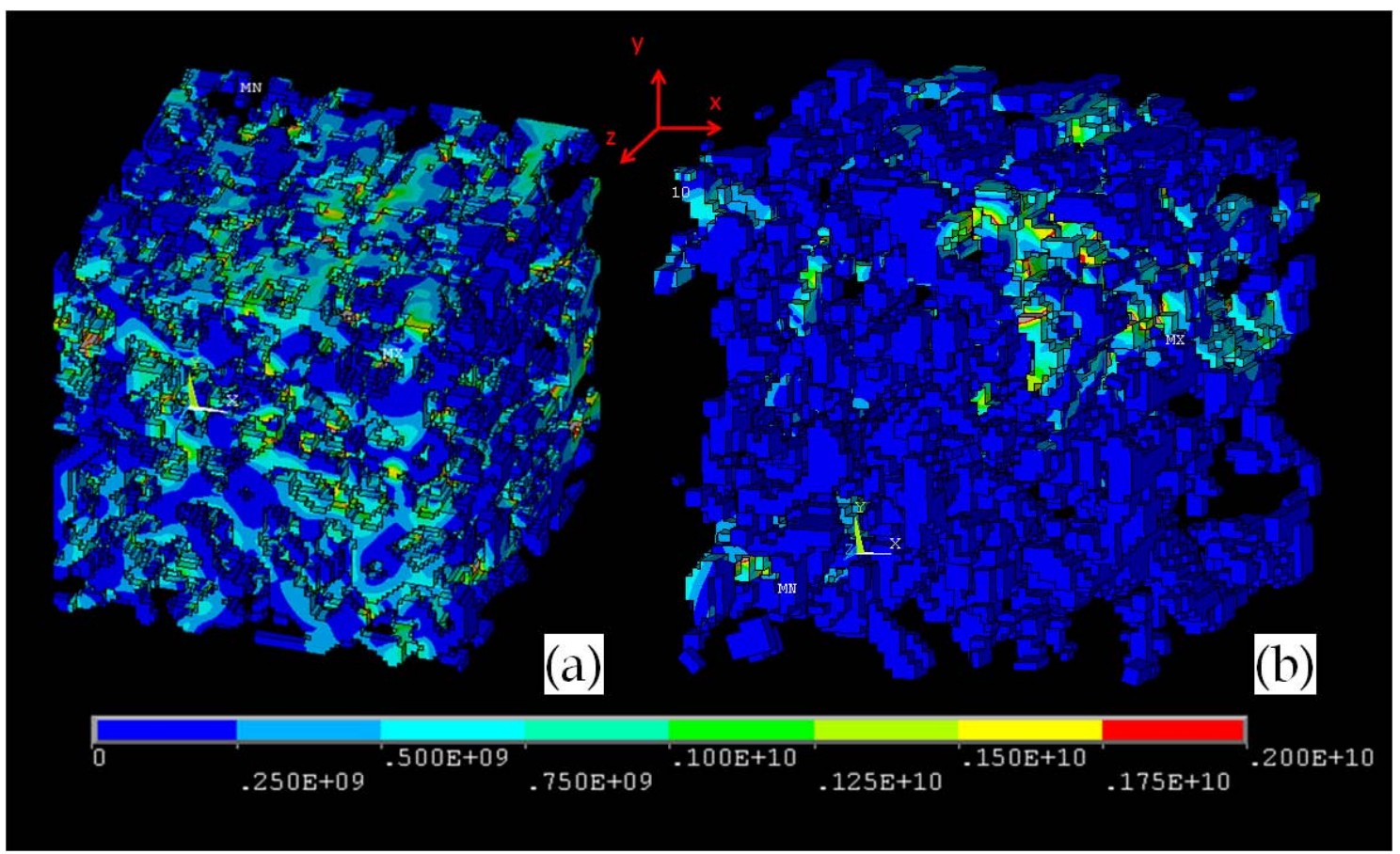

Figure 3.7. Nodal stresses $(\mathrm{Pa})$ parametric reconstruction calculated from the simulation of trabecular bone samples under compressive conditions in the z-axis. Note the homogeneous stress distribution assumed by the whole structure in the healthy bone of (a). The trabecular bone sample shown in (b) has a localized and heterogeneous stress-concentration due to microarchitecture deterioration.

In the cancellous bone structure, the mechanical stress tends to concentrate around the trabecular bone loops. The efficient propagation of forces from rods to surfaces and viceversa has a key role in the resistance to structure failure and bone fracture. In figure 3.8a, an example of uniform pressure applied uniaxially to a trabecular bone structure may be appreciated. However, despite the initial uniformity of the external conditions, the propagation of stresses inside the microarchitecture is disorganized and the superior part tends to deform more than the inferior, as can be observed in figure $3.8 \mathrm{~b}$. All these effects are the consequence of a heterogeneous pore distribution in the structure. 


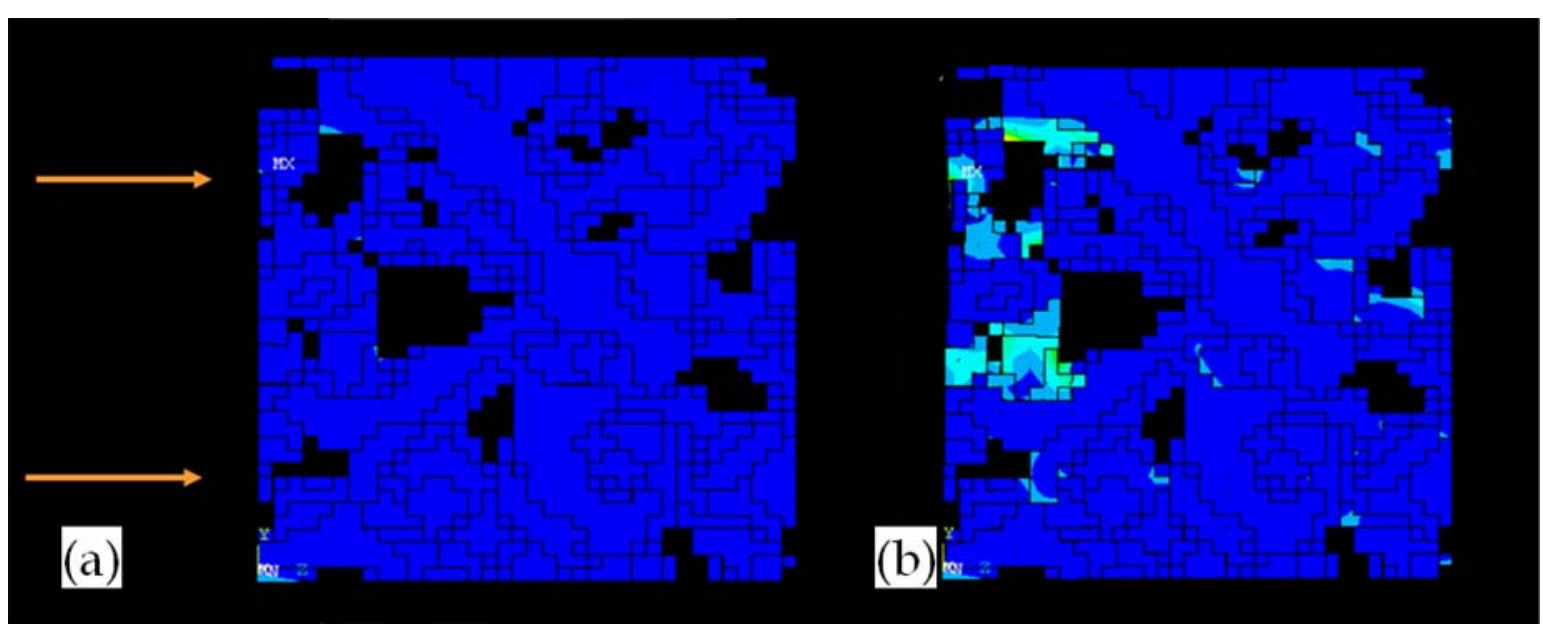

Figure 3.8. Application of a uniaxial and uniform pressure to one side of a trabecular bone sample to be simulated (a). In (b), the resulting stress parametric map combined with displacements information. Note the stress concentration around the trabecular bone pores and also the higher structure deformity in the upper region.

\section{Elastic modulus in the healthy bone}

Significant differences were found between male and female subjects for the results of $E_{a p p}$ in all of the three space directions $(p=0.006, p=0.007, p=0.01$; $\left.E_{a p p} X, E_{a p p} Y, E_{a p p} Z\right)$, with lower values in females, as it can be observed in table 3.5 and figure 3.9. Age was not influencing the results for the elastic modulus $\left(E_{a p p} X, \mathrm{r}=-0.01, \mathrm{p}=0.96 ; E_{a p p} Y, \mathrm{r}=-0.28, \mathrm{p}=0.22 ; E_{a p p} Z, \mathrm{r}=-0.18, \mathrm{p}=0.26\right)$.

\begin{tabular}{|c|c|c|c|c|}
\hline $\begin{array}{c}\text { Young's } \\
\text { modulus }\end{array}$ & $\begin{array}{c}\text { Men } \\
(\mathbf{n = 1 9 )}\end{array}$ & $\begin{array}{c}\text { Women } \\
(\mathbf{n = 2 1})\end{array}$ & p-value & $\begin{array}{c}\text { Total } \\
(\mathbf{n = 4 0 )}\end{array}$ \\
\hline Eapp $_{\text {X }}[\mathrm{MPa}]$ & $\begin{array}{c}136.91 \pm \\
39.80\end{array}$ & $61.75 \pm 24.52$ & 0.006 & $99.33 \pm 51.10$ \\
\hline $\mathrm{E}_{\text {app }} \mathrm{Y}[\mathrm{MPa}]$ & $\begin{array}{c}138.01 \pm \\
34.96\end{array}$ & $59.43 \pm 26.03$ & 0.007 & $98.72 \pm 51.88$ \\
\hline $\mathrm{E}_{\text {app }} \mathrm{Z}[\mathrm{MPa}]$ & $\begin{array}{c}241.58 \pm \\
122.34\end{array}$ & $\begin{array}{c}143.02 \pm \\
113.78\end{array}$ & 0.01 & $\begin{array}{c}189.84 \pm \\
126.62\end{array}$ \\
\hline
\end{tabular}

Table 3.5. Results Young's modulus parameter extracted from the compression simulations in the three directions of space. 


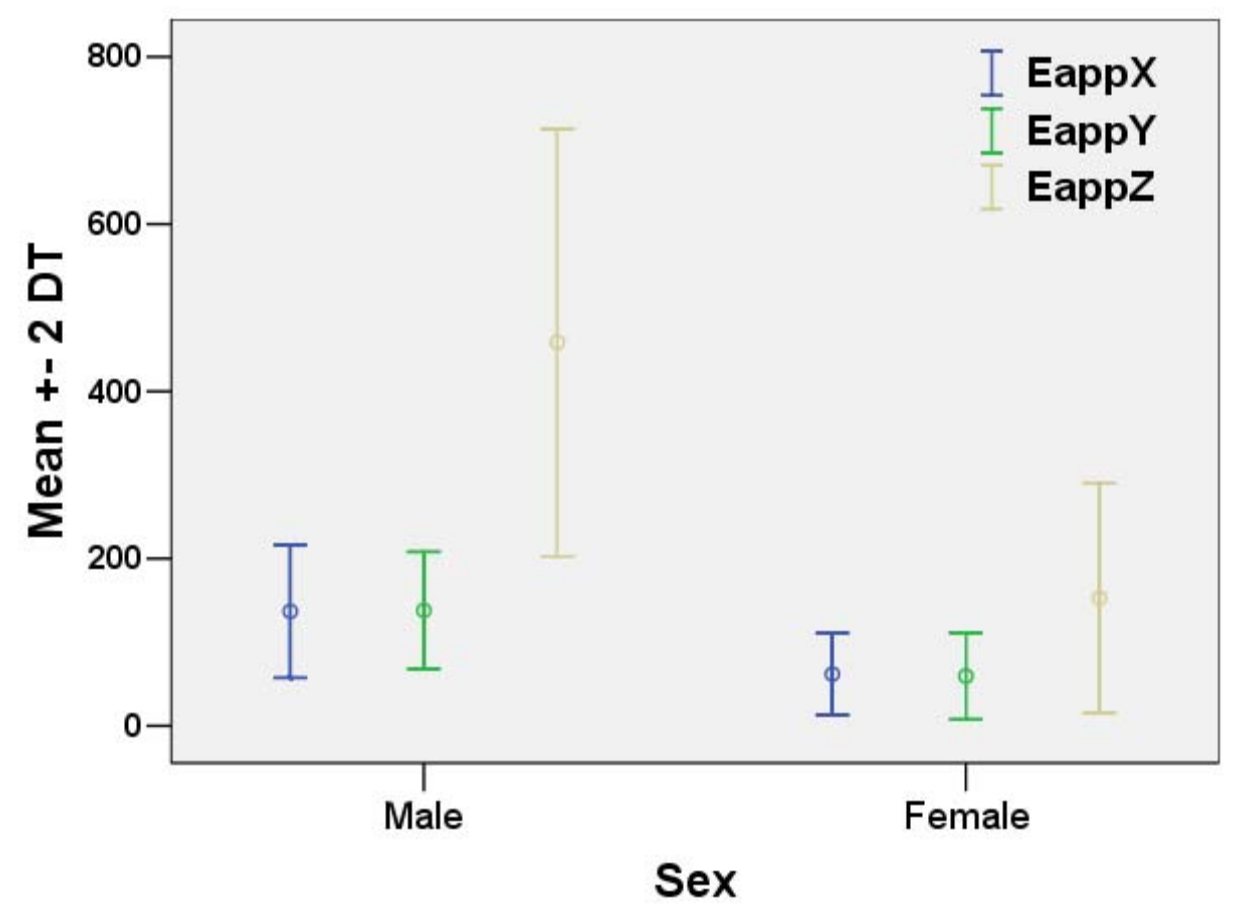

Figure 3.9. Results of the Young's modulus calculation from FE compression simulations in the three directions of space $\left(E_{a p p} X, E_{a p p} Y, E_{a p p} Z\right)$.

\section{- Discussion:}

Different elastic modulus were obtained for the different orientations, reinforcing the results obtained by means of 3D-MIL and 3D-Tensor Scale anisotropy analysis. The $E_{a p p} Z$ results, which correspond to the elastic modulus in the radius longitudinal direction, were significantly higher compared to the obtained in the transverse directions. These results are similar to those obtained by Geraets et al. [111].

Mechanical 3D analysis of complex structures by FE modeling has become a well established method in engineering. The use of this tool has grown widely in the last years in bioengineering medical science, mainly due to the improvement of clinical imaging CT and MR scanners. The higher spatial resolutions achieved with 3 Tesla MR scanners allow for the development of 3D reconstructions of tissues with the possibility of simulation in defined conditions. Several studies $[73,98,100]$ have demonstrated the potential of the 
FE method application in combination with image processing techniques and voxel-based FE meshes to test the elasticity and quality of the trabecular bone.

In our experiment, simulated mechanical tests were applied to the in vivo trabecular bone reconstructions using the FE method with voxel-meshed structures. Accurate mechanical simulations are achieved by using a direct conversion from the voxels of the $3 \mathrm{D}$ reconstruction to the brick hexahedron elements of the FE model. Gender showed a significant influence on $E_{\text {app }}$ results in any direction, being the results greater in men, in accordance with expected [113].

We found that the mean $E_{a p p} Z$ obtained in our series was lower than the value obtained in [98] for uniaxial compression (190 $\pm 127 \mathrm{MPa}$ vs. $2050 \pm 590 \mathrm{MPa}$, respectively). Although the mechanical analysis presented in [98] is very similar to the one exposed in the present thesis, the methodology described herein presented some differences in acquisition technique regarding main magnetic field intensity ( 3 Tesla vs. 1.5 Tesla), contrast sequence parameters ( $\mathrm{TE}=5 \mathrm{~ms}$, $\mathrm{TR}=16 \mathrm{~ms}, \mathrm{FA}=25^{\circ}$ vs. $\left.\mathrm{TE}=5.6 \mathrm{~ms}, \mathrm{TR}=29 \mathrm{~ms}, \mathrm{FA}=30^{\circ}\right)$, slice thickness $(180 \mu \mathrm{m}$ vs. $500 \mu \mathrm{m}$ ) and image post-processing (a sub-voxel processing algorithm was implemented in our study). Müller and Rüegsegger [114] obtained a compressive $E_{a p p} Z$ of the cancellous bone of the radius of $564 \mathrm{MPa}$ using high resolution CT with a slice thickness of $480 \mu \mathrm{m}$ and the application of the FE method by tetrahedrons. Therefore, use of different acquisition techniques, image spatial resolution and processing methods clearly influence the $E_{a p p}$ values. Standardization is therefore needed in this analysis.

Influence of VOI dimensions in elastic modulus results and their morphology relationships

The main limitations in the application of the FE method to trabecular bone meshes come from the high computational cost that supposes the calculus of the solution of the linear system of equations (24 equations per voxel). In order 
to minimize the computational burden, a representative VOI may be chosen as small as possible if provides reliable results.

However, after acquiring the results for the two different regions analyzed, it was concluded that the volume of analyzed bone had an influence on the elastic results. Although the statistical significant difference between male and female results for $E_{a p p} Z$ calculated in the small VOI $(\mathrm{p}=0.002)$ was maintained, the reliability of results was reduced. A statistically significant difference $(p<0.001)$ between $E_{a p p} Z$ results was obtained for the large VOI with those obtained for the small VOI $(189.84 \pm 41.17 \mathrm{MPa}$ vs. $81.56 \pm 31.61 \mathrm{MPa}$, mean $\pm \mathrm{SD}$, large vs. small VOI, respectively) was obtained. Differences between $E_{\text {app }} Z$ results for the two different analyzed volumes become greater when the values of the elastic parameter grow, as can be seen in the Bland-Altman plot (figure 3.10).

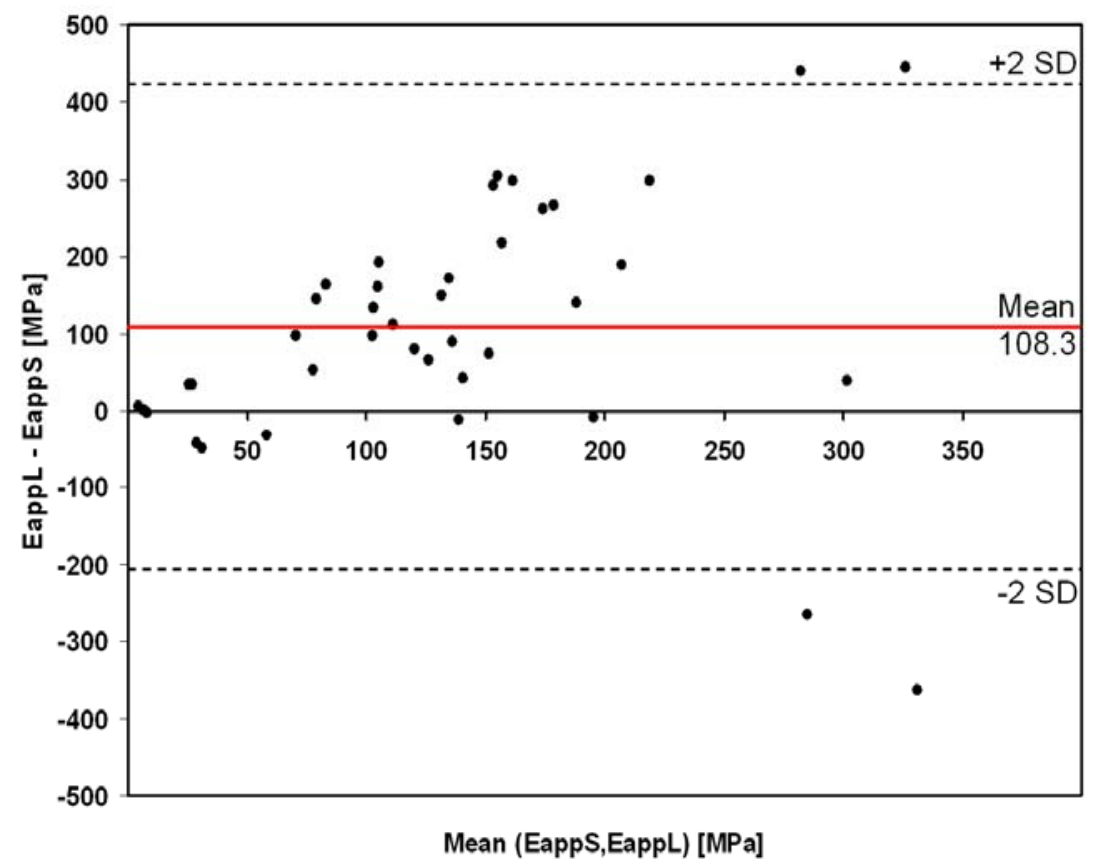

Figure 3.10. Bland-Altman plot shows results of apparent elastic modulus for large volume $\left(E_{\text {app }} \mathrm{S}\right)$ and small volume $\left(\mathrm{E}_{\mathrm{app}} \mathrm{L}\right)$ analyzed [66].

In the present work, two different cancellous bone segmented volumes were tested $(1.5 \times 1.5 \times 1 \mathrm{~cm}$ vs. $0.5 \times 0.5 \times 1 \mathrm{~cm})$ and it was found that the elastic results applied to the large sample volumes of trabecular bone better characterize its properties, with significant differences between male and female subjects for 
the $E_{a p p} Z$ parameter. However, we showed that the goodness of the results diminishes as the restricted volume dimensions decrease.

A statistically significant relationship was found between the elastic modulus and the square of $B V / T V$ calculation $(\mathrm{r}=0.968, \mathrm{p}<0.001)$, reflecting the high linear function between mechanical and morphology parameters, as it can be observed in figure 3.11. However, the use of small volumes for elasticity calculation provided a non-normal distribution and gave no statistical correlation $(\rho=0.185, \mathrm{p}=0.25)$ with the $B V / T V^{2}$ parameter. The behavior of the relationship between elastic modules calculated from the two different volumes and $B V / T V^{2}$ is also observed in figure 3.11 , where a linear performance is shown for the large volume while no goodness of fit is found in the small volume estimation.

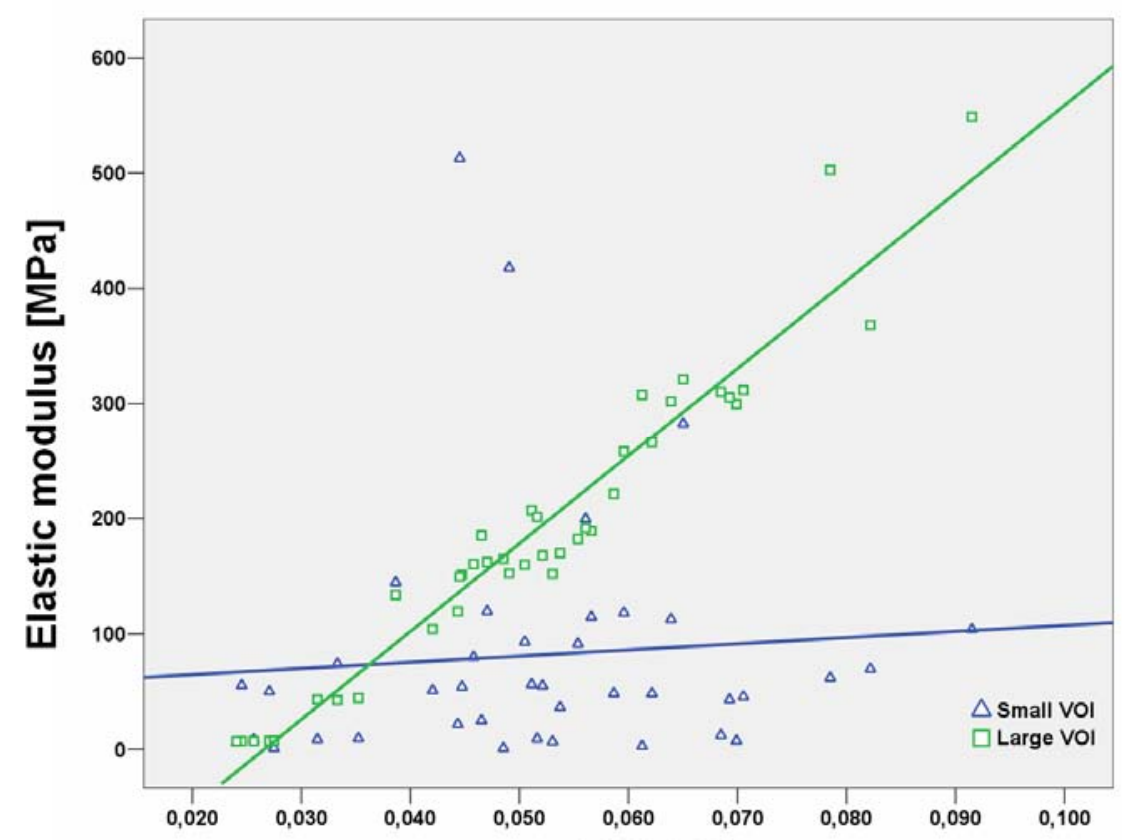

$(\mathrm{BV} / \mathrm{TV})^{2}$

Figure 3.11. Different behaviors of the correlation of $E_{a p p} Z$ with $B V / T V^{2}$ can be observed depending on the analyzed VOI [66]. Small VOI samples present no relationship with $B V / T V^{2}$ parameter. Large VOI samples have a linear behavior and high correlation between $E_{a p p} Z$ and $B V / T V^{2}$.

When comparing in vivo relationships between elastic and morphology results, a high correlation for both genders between longitudinal elastic modulus (mechanical parameter) and the square of $B V / T V$ (morphology parameter) was 
found, agreeing with ex vivo tests in the relationship between $E_{a p p} Z$ and $B V / T V$ $[115,116]$. This behavior agrees the theory $[117,118]$ about the existence of a strong relationship between the elastic modulus and the square of porosity of a material in the elastic regime. This observed exponential dependence for the compressive modulus takes the form

$$
\frac{E}{E_{b}}=C \cdot(B V / T V)^{2}
$$

where $E_{b}$ is the elastic modulus of the bulk (compact bone) which has been set to $10 \mathrm{GPa}$ (section 2.5.1). From the results, a predicted value of $C=0.75$ was obtained giving the linear relationship between the square of $B V / T V$ and the $E_{\text {app }} Z$.

\subsection{Assessment of morphometry and mechanical alterations under pathological conditions in osteoporosis disease.}

In this section, the methodology developed and implemented in the present thesis is applied to the group of female patients with osteoporosis introduced in section 2.7. The results are compared to an age and gender matched group of healthy controls. Finally, the relationships between the calculated parameters and the BMD values in the osteoporotic patients are also presented.

\subsubsection{Morphometry results in osteoporotic patients}

\section{Morphology}

The algorithms for cancellous bone morphology characterization were applied to the group of female patients with osteoporosis in order to evaluate the alterations under pathological conditions. 
The results of the analysis are outlined in table 3.6. The Tb.Th and Tb.Sp were significantly influenced by the trabecular bone status, with a decreased $T b . T h$ $(\mathrm{p}=0.03)$ and an increased Tb.Sp $(\mathrm{p}=0.05)$ in the osteoporotic patients in comparison to the group of controls. The BV/TV and Tb.N showed no statistical differences ( $p=0.45$ and $p=0.26$, respectively) between both groups analyzed. However, a slight tendency to an increased $B V / T V$ was observed in the healthy subjects.

\begin{tabular}{|c|c|c|c|}
\hline Parameter & $\begin{array}{c}\text { Healthy } \\
(\mathbf{n}=\mathbf{1 0})\end{array}$ & $\begin{array}{c}\text { Osteoporosis } \\
\mathbf{( n = 2 0 )}\end{array}$ & p-value \\
\hline BV/TV $[\mathbf{x} \mathbf{1 0 0} \% \mathbf{0}]$ & $0.22 \pm 0.03$ & $0.21 \pm 0.03$ & 0.45 \\
\hline Tb.Th $[\boldsymbol{\mu m}]$ & $192.9 \pm 4.4$ & $188.5 \pm 4.7$ & 0.03 \\
\hline Tb.Sp $[\boldsymbol{\mu m}]$ & $998.0 \pm 106.7$ & $1086.8 \pm 94.3$ & 0.05 \\
\hline Tb.N $\left[\mathbf{1 0}^{-\mathbf{3}} \boldsymbol{\mu m}^{-\mathbf{1}}\right]$ & $1.11 \pm 0.14$ & $1.19 \pm 0.16$ & 0.26 \\
\hline
\end{tabular}

Table 3.6. Morphology results in the group of osteoporotic patients and healthy subjects.

The differences in the morphology parameters calculated herein may be visually depicted in figure 3.12 . 

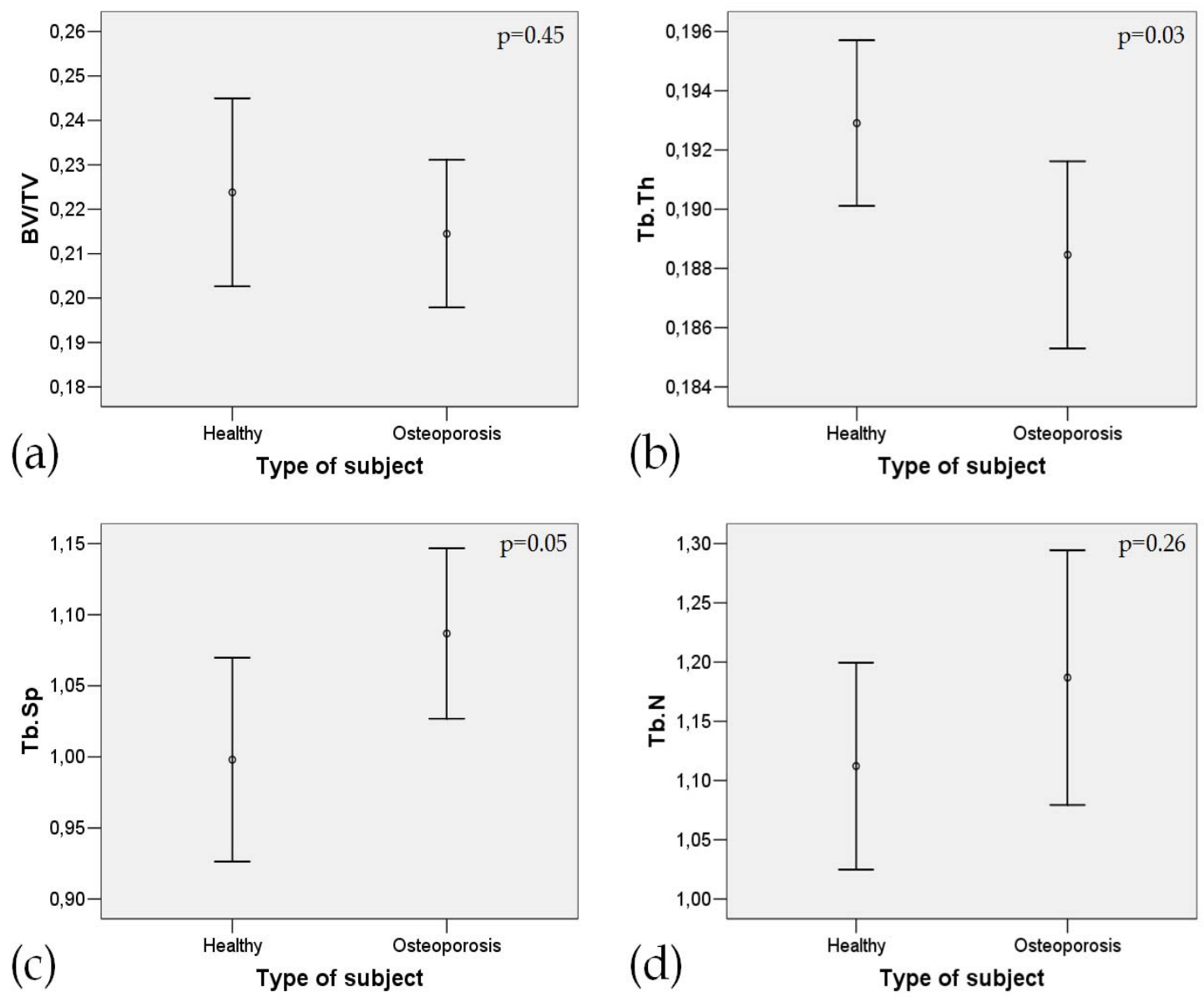

Figure 3.12. Results of the morphology parameters calculated in the healthy and osteoporotic populations. Note the slight differences for the results in (a) and (d) with considerable ranges overlap (BV/TV and Tb.N, respectively). In (b) and (c), significant differences exist between groups although groups are not fully separated (Tb.Th and $T b . S p$, respectively).

A significant relationship was found between $T b . S p$ and age for the subjects analyzed $(r=0.44, p=0.03)$. A graphical illustration of this dependency may be appreciated in figure 3.13. No significant relationships with age were detected for the rest of the morphological indexes calculated $(r=-0.24, p=0.28 ; r=-0.37$, $\mathrm{p}=0.09 ; \mathrm{r}=0.39, \mathrm{p}=0.09 . B V / T V ; T b . T h ; T b . N$, respectively). 


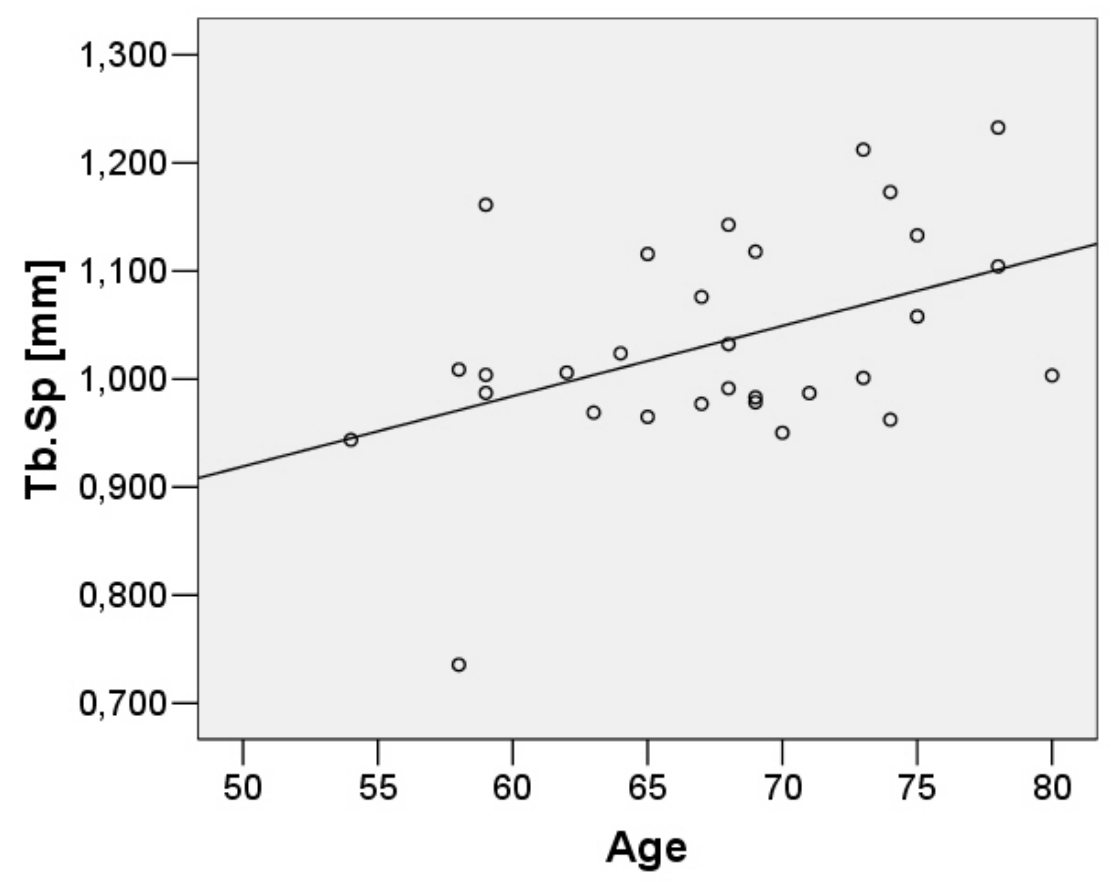

Figure 3.13. Linear regression showing the relationship of

$T b . S p$ with age for both healthy and osteoporotic populations $(\mathrm{r}=0.44, \mathrm{p}=0.03)$.

Since age seemed to be significantly influencing the Tb.Sp values and slightly affecting Tb.Th and Tb.N results, influence of osteoporosis conditions in morphology parameters was re-analyzed with a statistical correction for age. A linear general model considering the age as a co-variable was used to analyze the morphology parameters. The corrected method provided p-values of 0.28 , 0.04, 0.01 and 0.11 for $B V / T V, T b . T h, T b . S p$ and Tb.N, respectively. The degree of signification was reduced for $T b . T h$ and increased for $T b . S p$, with respected to the analysis without age influence correction.

- Discussion:

These age dependencies of the calculated scale indicators could be probably related to the natural resorptive processes of osteoclasts after menopause, which directly affect the structure in terms of rod erosion. Nevertheless, as observed, the $B V / T V$ does not seem to be influenced by age. These differences in the behavior of the morphological parameters can be explained by the tendency to present large bone quantities in the junctions of rods and plates, which account more for $B V / T V$, while there exists a progressive erosion of bone 
rods which directly influences $T b . T h$. This mechanism is in some cases referred as 'homogeneous thinning' [119].

Although some significant differences exist for the morphological indicators, the sensitivity to the trabeculae abnormalities is limited. Some studies have shown the need of combining morphology results with other indicators such as topology or elasticity-related indexes in order to increase the prediction of trabecular bone quality in comparison to the calculation of morphology characteristics alone [116].

\section{Complexity}

Very relevant data was obtained from the application of the algorithms for trabecular bone fractal characterization to the group of patients with osteoporosis. Calculated values for $D^{2 D}$ and $D^{3 D}$ may be specified in table 3.7. Significant differences were found for the $D^{3 D}$ parameter between the healthy and osteoporotic populations $(\mathrm{p}<0.001)$ with higher values in the healthy group than in the osteoporotic ( $2.33 \pm 0.04$ vs. $2.27 \pm 0.03$, healthy vs. osteoporosis). Although with less statistical significance, a clear dissimilarity was observed between the healthy and osteoporotic patients $(\mathrm{p}=0.04)$ for the $D^{2 D}$ parameter, with also higher values in the healthy group than in the osteoporotic population (1.55 \pm 0.03 vs. $1.50 \pm 0.06$, healthy vs. osteoporosis). However, variances showed a trend to dissimilarity in this case $(p=0.08)$.

\begin{tabular}{|c|c|c|c|}
\hline Parameter & $\begin{array}{c}\text { Healthy } \\
\mathbf{( n = 1 0 )}\end{array}$ & $\begin{array}{c}\text { Osteoporosis } \\
(\mathbf{n = 2 0 )}\end{array}$ & p-value \\
\hline D $^{2 D}$ & $1.55 \pm 0.03$ & $1.50 \pm 0.06$ & 0.04 \\
\hline D $^{3 D}$ & $2.33 \pm 0.04$ & $2.27 \pm 0.03$ & 0.001 \\
\hline
\end{tabular}

Table 3.7. Fractal dimension results for the 2D and 3D approaches in the group of osteoporotic patients and healthy subjects.

A representation of the results distribution for $D^{2 D}$ and $D^{3 D}$ in the healthy and osteoporotic groups can be observed in figure 3.14. 

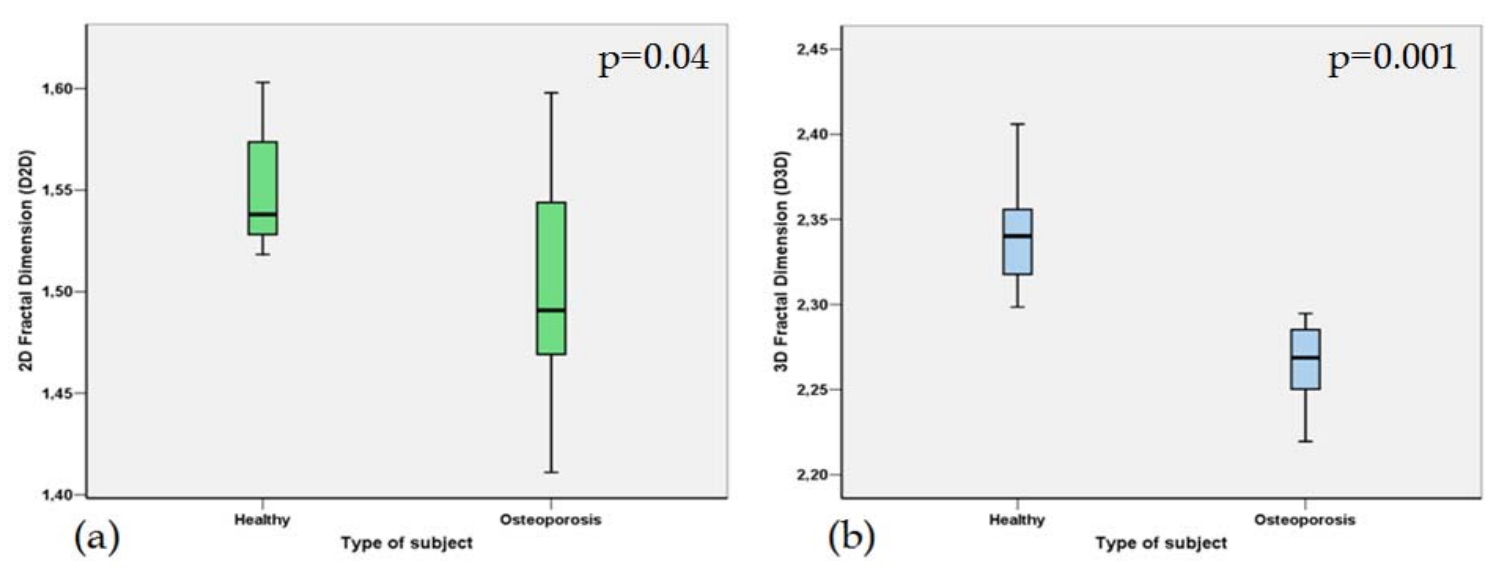

Figure 3.14. Box-whisker plots showing the differences between the healthy and osteoporotic populations for the $D^{2 D}$ (a) and $D^{3 D}(\mathrm{~b})$ parameters. Different data dispersion exists for the $D^{2 D}$ parameter between both groups.

No significant relationships were found between $D^{2 D}, D^{3 D}$ and age $(\mathrm{r}=-0.02$, $\mathrm{p}=0.94$ and $\mathrm{r}=-0.31, \mathrm{p}=0.18 ; D^{2 D}$ vs. age, $D^{3 D}$ vs. age, respectively).

- Discussion:

In this study, the feasibility of 2D and 3D fractal analysis methods for the in vivo characterization of the trabecular bone structural complexity was assessed. Image processing techniques and box-counting algorithms were implemented in order to quantify the $D^{2 D}$ and $D^{3 D}$ parameters of the cancellous bone from high-spatial resolution 3 T MR acquisitions of the distal radius metaphysis. The $D^{2 D}$ and $D^{3 D}$ parameters have been found to significantly differentiate between healthy and osteoporosis conditions. Furthermore, $D^{3 D}$ has shown better discrimination $(p<0.001)$ between the healthy volunteers and the osteoporotic patients than $D^{2 D}(\mathrm{p}=0.04)$.

The calculation of $D^{3 D}$ values from high-spatial resolution MR measurements of trabecular bone was found to be feasible if applied after proper image processing and efficient contour detection algorithms. A low processing time was achieved easing the integration of the method in an automated trabecular bone fractal characterization processing sequence. To the doctorand knowledge, the application of 3D fractal measurements to the study of trabecular bone from MR acquisitions has not been previously investigated. 
The values obtained for the $D^{3 D}$ parameter were significantly different between the healthy and osteoporosis groups $(\mathrm{p}<0.001)$, with lower values in the osteoporotic population (figure 3.14b). In this sense, Ito et al. obtained significant differences for the MDCT-derived $D^{3 D}$ parameter between women with and without history of osteoporotic fractures $(p<0.001)$ [51]. Although with less significance, the $D^{2 D}$ results showed a clear statistical differentiation between both groups $(p=0.04)$ (figure 3.14a). This finding is comparable to the obtained by Majumdar et al. where the $D^{2 D}$ parameter was found to be slightly different between healthy and osteoporotic post-menopausal populations $(p=0.01)$ with lower values in the women with osteoporosis $(1.43 \pm 0.11$ vs. 1.55 \pm 0.03 , healthy; $1.34 \pm 0.17$ vs. $1.50 \pm 0.06$, osteoporosis; Majumdar et al. vs. our results, respectively) [55]. These significant differences were detected even with the large slice thickness of their acquisition, which was of $700 \mu \mathrm{m}$ at a $1.5 \mathrm{~T}$ scanner.

On the contrary, Hudelmaier et al. did not observe significant differences between the results of the $D^{2 D}$ in a group of healthy volunteers compared to a group of patients with osteoporosis $(1.72 \pm 0.03$ vs. $1.71 \pm 0.03, p>0.05$; healthy vs. osteoporosis, respectively) [120]. Although their in-plane spatial resolution (156 $\mu \mathrm{m})$ was exactly the same than the used by Majumdar et al. [55], slice thickness was smaller $(300 \mu \mathrm{m})$. In the calcaneus, Link et al. found significant differences in the $D^{2 D}$ parameter between healthy and osteoporotic post-menopausal women $(p=0.007)$ with lower values in the osteoporosis cases $(1.66 \pm 0.06$ vs. $1.61 \pm 0.04$; healthy vs. osteoporosis, respectively) [53].

As several different definitions exist for the fractal dimension parameter, the used algorithm or method must always be carefully specified. Some authors found similar values with MR but several methodological differences exist between studies and an effective comparison of the results is not possible [121]. In this sense, in the works mentioned above $[53,55,120]$ the box-counting algorithm was used for $D^{2 D}$ calculation while the analysis of Pothuaud et al. was based on the Fractional Brownian Motion (FBM) model and the evaluation 
of porosity was implemented by numerical dilation and removal processes to the $2 \mathrm{D}$ trabecular bone images.

Our results suggest that for the fractal characterization of bone trabeculae complexity, a 3D approach should be used instead of the 2D box-counting algorithm. The data distribution variance in healthy and osteoporotic populations is more uniform for the $3 \mathrm{D}$ than in the $2 \mathrm{D}$ approach. In $2 \mathrm{D}$, more dispersion is obtained in the patients group. The different variances observed in both parameters, with less data dispersion in the 3D approach, expresses that the $\mathrm{D}^{3 \mathrm{D}}$ is more accurate than the $\mathrm{D}^{2 \mathrm{D}}$ data for osteoporosis diagnosis.

The principal orientation of the trabecular bone in the radius corresponds to the longitudinal dimension [111]. If axial images are acquired with anisotropic spatial resolution, that is, with a slice thickness larger than the in-plane highspatial resolution, the main orientation of the trabecular bone is being clearly underestimated. Even more, if a 2D approach is used for the fractal characterization, with the results averaged with all the slices, the main alignment direction of the trabeculae is also not taken into consideration. The combination of high-spatial isotropic resolutions in acquisition of the images combined with a 3D post-processing algorithm seems to be the most appropriate configuration for an efficient fractal characterization of the trabecular bone. Compared with $1.5 \mathrm{~T}$, the high-spatial resolutions images achievable with $3 \mathrm{~T}$ magnets in all three directions together with the increased SNR have allowed the implementation of $D^{3 D}$ quantification algorithms.

\section{Topology}

The topology results showed significant differences for the TEI and SCR between the group of osteoporotic patients and the corresponding healthy group ( $\mathrm{p}<0.001$ and $\mathrm{p}=0.001, T E I$ and $S C R$, respectively). As it may be directly extracted from table 3.8, increased erosion values in the case of osteoporotic patients $(11.17 \pm 4.81$ vs. $17.78 \pm 4.93$ for TEI; $0.47 \pm 0.16$ vs. $0.31 \pm 0.14$ for SCR; healthy vs. osteoporosis, respectively) were detected. 


\begin{tabular}{|c|c|c|c|}
\hline Group & $\begin{array}{c}\text { Healthy } \\
\mathbf{( n = 1 0 )}\end{array}$ & $\begin{array}{c}\text { Osteoporosis } \\
(\mathbf{n}=\mathbf{2 0})\end{array}$ & p-value \\
\hline TEI & $11.17 \pm 4.81$ & $17.78 \pm 4.93$ & 0.001 \\
\hline SCR & $0.47 \pm 0.16$ & $0.31 \pm 0.14$ & 0.001 \\
\hline
\end{tabular}

Table 3.8. Topology results (TEI and SCR) for the 2D and 3D approaches in the group of osteoporotic patients and healthy subjects.

In figure 3.15, the results of the TEI and SCR are depicted for a visual assessment of the differences between the osteoporosis and healthy populations.
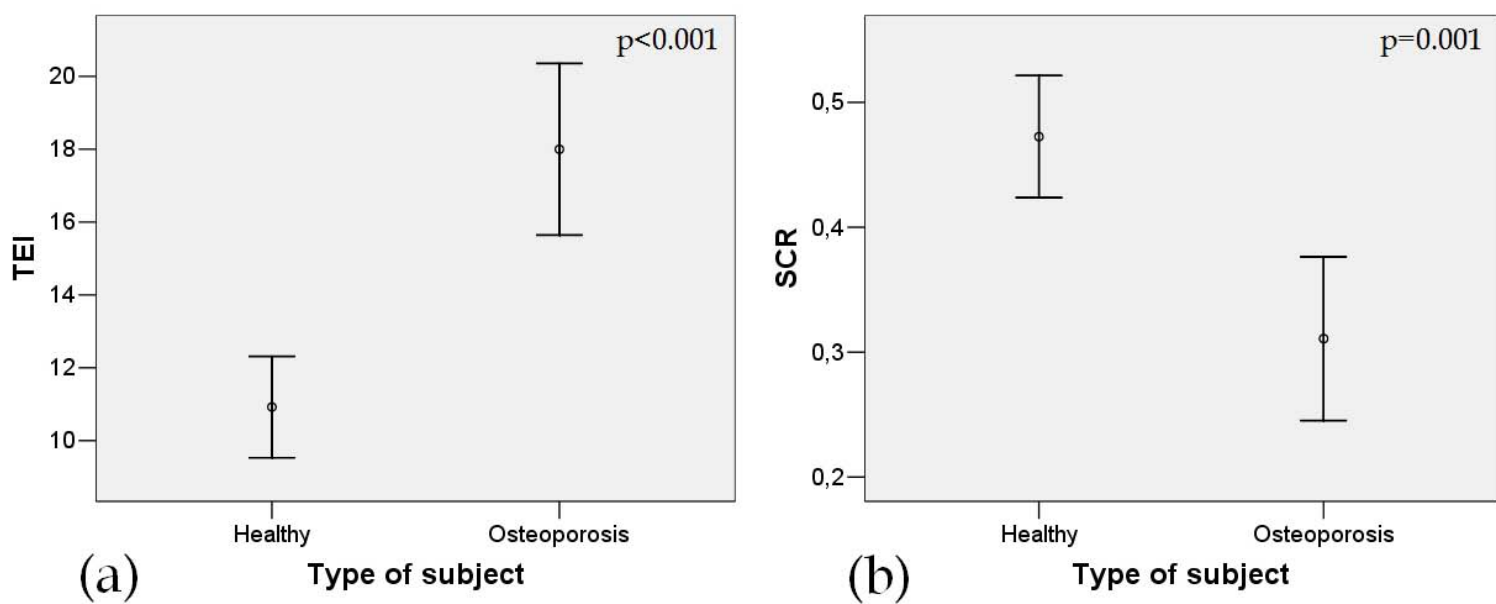

Figure 3.15. Error bar plots showing the differences between the healthy and osteoporotic populations for the TEI (a) and SCR (b) parameters.

No relationships were detected between age and the topology parameters $(\mathrm{r}=0.04, \mathrm{p}=0.85, T E I ; \mathrm{r}=-0.01, \mathrm{p}=0.95, S C R)$.

\section{- Discussion:}

The potential of DTA as a means to quantitatively characterize the $3 \mathrm{D}$ architecture of cancellous bone networks is demonstrated. An important property of the proposed method is its ability to uniquely classify each bone voxel after binarization and skeletonization, therefore providing local connectivity information. The known conversion of plates to rods during normal aging, especially in osteoporosis, may be followed quantitatively by 
measuring the $S C R$, or an erosion index, as composite parameters directly amenable from the analysis $[70,88]$.

The TEI emphasizes the qualitative trends of erosion (rod versus plate-likeness). It has been shown that erosion of trabeculae results in bone plates changing to rods, which eventually become disconnected [119].

Since all the voxels of the skeletonized trabecular bone reconstruction are classified by the DTA algorithm in terms of spatial localization and topology class, parametric maps of the topological classification can be also composed in order to qualitatively assess the fabric topology. In figure 3.16, parametric reconstructions showing topology classification may be observed. The main differences in the topology classification at both structures may be graphically assessed by the colors of each class. An increased number of orange, red and brown voxels (related to bone surfaces) may be observed for the healthy subject in figure 3.16a when compared to the classification obtained in the patient with osteoporosis of figure 3.16b, where more blue voxels (related to bone curves) exist.

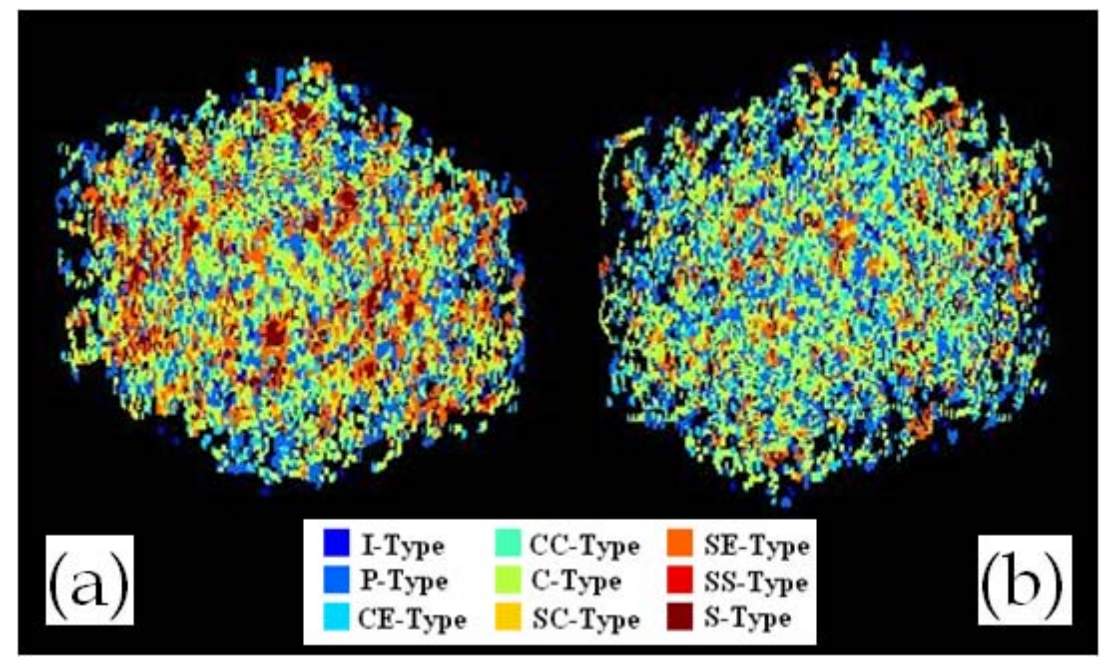

Figure 3.16. Topological classification of trabecular bone. In (a), topology classified reconstruction of a trabecular bone from a healthy subject. In (b), topological reconstruction of a trabecular bone from a patient with osteoporosis. 


\section{Anisotropy}

The algorithms developed for the analysis of anisotropy and structural orientation were applied to the population of patients with osteoporosis.

Both 3D-MIL and 3D-Tensor Scale methods for the assessment of anisotropy were used in the case of patients with osteoporosis. Significant differences were obtained between the $D A$ parameter calculated using both techniques, with higher values for the 3D-Tensor Scale method $(p=0.04)$. No differences existed for the results of $\theta_{a}$ using both algorithms $(\mathrm{p}=0.47)$.

When comparing the $D A$ results obtained in the osteoporotic patients with the calculated values in a group of age-matched healthy female subjects, both 3DMIL and 3D-Tensor Scale methods presented significant differences between the groups $(1.09 \pm 0.02$ vs. $1.11 \pm 0.02$, healthy vs. osteoporosis, $\mathrm{p}=0.02,3 \mathrm{D}-\mathrm{MIL}$; $1.11 \pm 0.02$ vs. $1.14 \pm 0.02$, healthy vs. osteoporosis, $\mathrm{p}<0.001,3 \mathrm{D}$-Tensor Scale), with a higher differentiation for the 3D-Tensor Scale method, as can be appreciated in figure 3.17. No significant differences were found for $\theta_{a}$ either for 3D-MIL or for 3D-Tensor scale method, with $\mathrm{p}=0.97$ and $\mathrm{p}=0.57$, respectively. Mean values are summarized in table 3.9.

\begin{tabular}{|c|c|c|c|c|}
\hline Method & Parameter & Healthy $\mathbf{( n = 1 0 )}$ & $\begin{array}{c}\text { Osteoporosis } \\
\mathbf{( n = 2 0 )}\end{array}$ & p-value \\
\hline \multirow{2}{*}{ 3D-MIL } & $\theta_{\mathrm{a}}$ & $72.61 \pm 11.50$ & $72.71 \pm 13.31$ & 0.97 \\
\cline { 2 - 5 } & $\mathrm{DA}$ & $1.09 \pm 0.02$ & $1.11 \pm 0.02$ & 0.02 \\
\hline \multirow{2}{*}{$\begin{array}{c}\text { 3D-Tensor } \\
\text { Scale }\end{array}$} & $\theta_{\mathrm{a}}$ & $68.51 \pm 14.58$ & $71.26 \pm 13.16$ & 0.57 \\
\cline { 2 - 5 } & DA & $1.11 \pm 0.02$ & $1.14 \pm 0.02$ & 0.001 \\
\hline
\end{tabular}

Table 3.9. Results of the $\theta_{a}$ and DA parameters after the application of 3D-MIL and 3D-Tensor Scale algorithms to both groups of healthy and osteoporotic subjects. 
MIL 3D

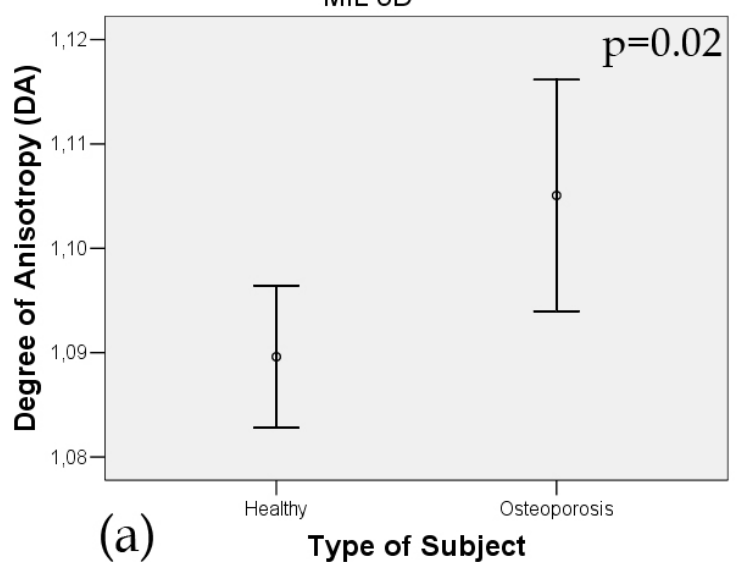

Tensor Scale 3D

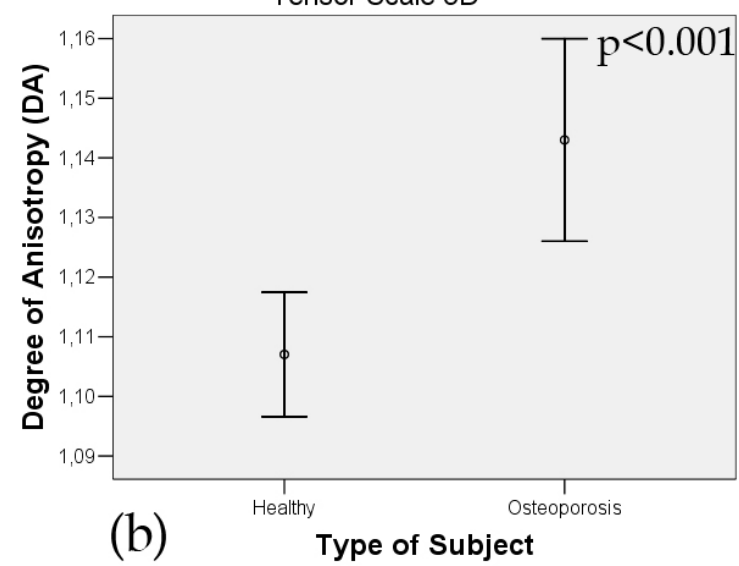

Figure 3.17. Results of the DA parameter in both healthy and osteoporotic populations. In (a), results obtained with the 3D-MIL algorithm. In (b), values calculated with the 3D-Tensor Scale algorithm.

- Discussion:

The initial hypothesis was that the $D A$ should be increased in patients with trabecular bone deterioration or osteoporosis. The main reason is explained by some published conclusions confirming the existence of a preferential loss of transverse trabeculae while longitudinal trabeculae tend to remain aligned in the progress of the disease [122].

Results showed a slight overlap for the 3D-MIL results, while a better differentiation between healthy and osteoporotic subjects exists for the results obtained with the 3D-Tensor Scale algorithm (figure 3.17). These differences between healthy subjects and osteoporotic patients are probably related to the increased loss of transverse trabeculae with respect to the principal direction as a mechanism of progressive bone atrophy. In fact, the preferential loss of transverse trabeculae appears to be a hallmark of osteoporosis occurring at other skeletal locations as well, such as in the proximal femur where subjects with hip fractures were found to have fewer trabeculae perpendicular to the major loading direction [110]. 


\subsubsection{Mechanical results in osteoporotic patients}

Young's modulus variations in osteoporosis

The calculated Young's modulus values are summarized in table 3.10. Significant differences between the healthy and osteoporosis groups were found for the $E_{a p p} X \quad(p=0.01)$ and $E_{a p p} Z(p=0.04)$ parameters, while no dissimilarities existed for $E_{\text {app }} Y(\mathrm{p}=0.43)$.

\begin{tabular}{|c|c|c|c|}
\hline Young's modulus & Healthy $(\mathbf{n = 1 0 )}$ & $\begin{array}{c}\text { Osteoporosis } \\
(\mathbf{n}=\mathbf{2 0})\end{array}$ & p-value \\
\hline Eapp $_{\text {X }}[\mathrm{MPa}]$ & $56.03 \pm 21.54$ & $20.55 \pm 24.66$ & 0.01 \\
\hline E $_{\text {app }} \mathbf{Y}[\mathrm{MPa}]$ & $54.22 \pm 21.77$ & $39.99 \pm 35.59$ & 0.43 \\
\hline $\mathrm{E}_{\text {app }} \mathbf{Z}[\mathrm{MPa}]$ & $114.72 \pm 106.94$ & $59.12 \pm 40.58$ & 0.04 \\
\hline
\end{tabular}

Table 3.10. Results of $\mathrm{E}_{\mathrm{app}}$ for the different axis where compressive conditions were applied.

No associations with age were found for the $E_{\text {app }}$ results $\left(\mathrm{r}=0.10, \mathrm{p}=0.69, E_{\text {app }} X\right.$; $\left.\mathrm{r}=-0.19, \mathrm{p}=0.46, E_{a p p} Y, \mathrm{r}=0.23, \mathrm{p}=0.38, E_{a p p} \mathrm{Z}\right)$.

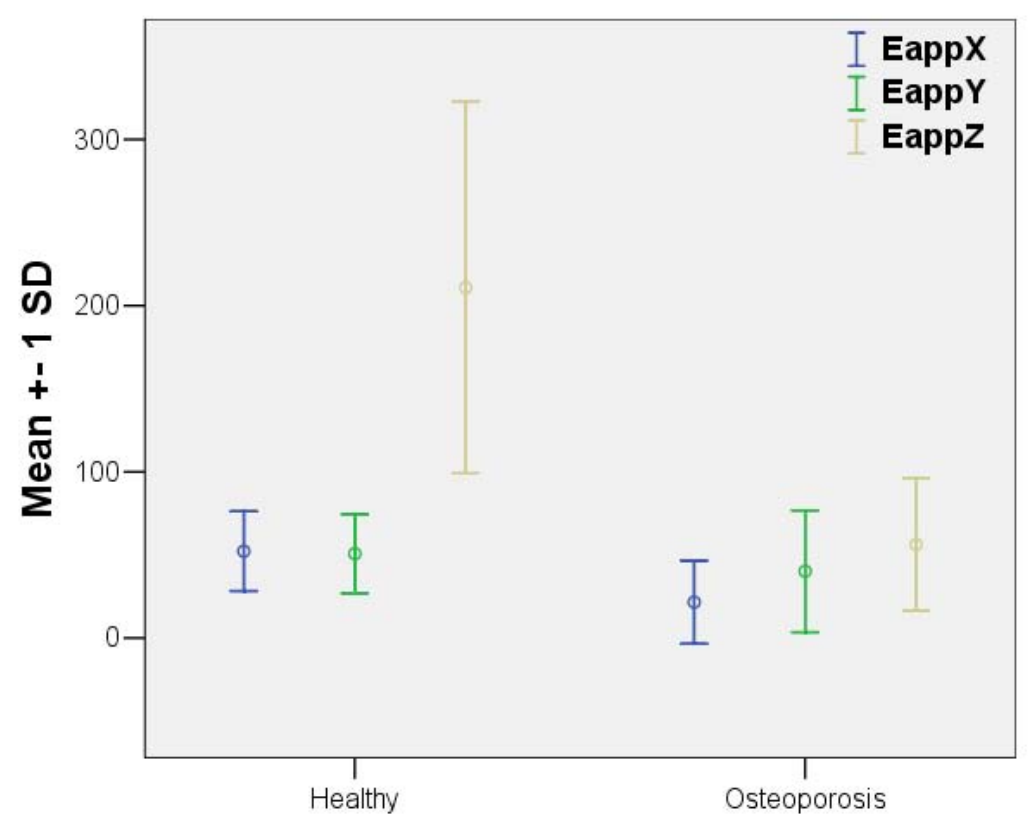

Figure 3.18. Results of the Eapp (MPa) in each one of the three space directions for the healthy and osteoporotic female populations. EappX and EappZ are significantly affected by the disease. 
- Discussion:

In the observed values for the osteoporotic subjects, elastic modulus results were approximately reduced in a $40 \%-60 \%$ with respect to the healthy values. The directional mechanics of bone may potentially play an important role in predicting biomechanics, as has been shown in vitro [123-125], and hence these properties and their relative changes may be useful for fracture prediction and assessing response to therapy.

As previously introduced, Mosekilde found that the decrease in mechanical competence upon bone loss in the vertebrae is exacerbated by preferential loss of transverse trabeculae, rendering the bone prone to failure by buckling [122]. Their results are consistent with those obtained in the present study. The differences between the normal and osteoporotic subjects are greater in the direction of the weaker modulus, implying that in osteoporosis, the thinner trabeculae are lost first. This is also consistent with results in the spine, where it was showed that horizontal thinner trabeculae are lost first with aging and osteoporosis [122]. Newitt et al. also obtained a significant reduction of the weaker elastic modulus in osteopenic conditions, with respect to healthy values [98]. These conclusions reinforce the results obtained in the current thesis, where the application of the $\mu \mathrm{FE}$ simulations as a mechanical characterization of trabecular bone microarchitecture has shown promising results.

\section{$\underline{\text { Role of the trabeculae skeleton in the Young's modulus results }}$}

The trabeculae skeleton defines the microarchitecture shape of the rods and plates distribution within the cancellous bone tissue. Its importance in the topology analysis for the detection of trabecular curves and surfaces and their connectivity has been exposed in section 3.2.1, where also a high sensitivity for the detection of trabecular bone deterioration mechanisms was observed.

In the current analysis, the influence of the trabecular bone skeleton in the mechanical response of trabecular structure was determined. In order to 
analyze such situation, the trabecular bone reconstructions from both healthy and osteoporotic populations and their corresponding skeletonized structures were simulated under uniaxial compression conditions in the $\mathrm{z}$-direction. No previous reported results were found in the literature of a similar analysis applied to trabecular bone reconstructions.

The results for the $E_{a p p} Z$ in the skeletonized structures were of $0.155 \pm 0.093 \mathrm{MPa}$ in the healthy subjects and of $0.041 \pm 0.036 \mathrm{MPa}$ in the case of patients with osteoporosis. Such results suppose a reduction of approximately 1000 times from the $E_{\text {app }} \mathrm{Z}$ values obtained in the non-skeletonized structures.

From these results, it can be concluded that the skeleton supposes just about a $0.14 \%$ of the overall $E_{a p p} Z$ in healthy subjects and a $0.69 \%$ in the case of patients with osteoporosis.

The skeleton contribution to the $E_{a p p} Z$ considering the complete structure is hence quite poor. These results give relevance to the way in which bone trabeculae are disposed, considering other factors like thicknesses, pore dimensions, contours definition and elements which act as stress concentrators and let the structure to support high stresses with linear strains.

In figure 3.19, examples of the stress intensity parametric results from the $\mu \mathrm{FE}$ simulations may be observed. 


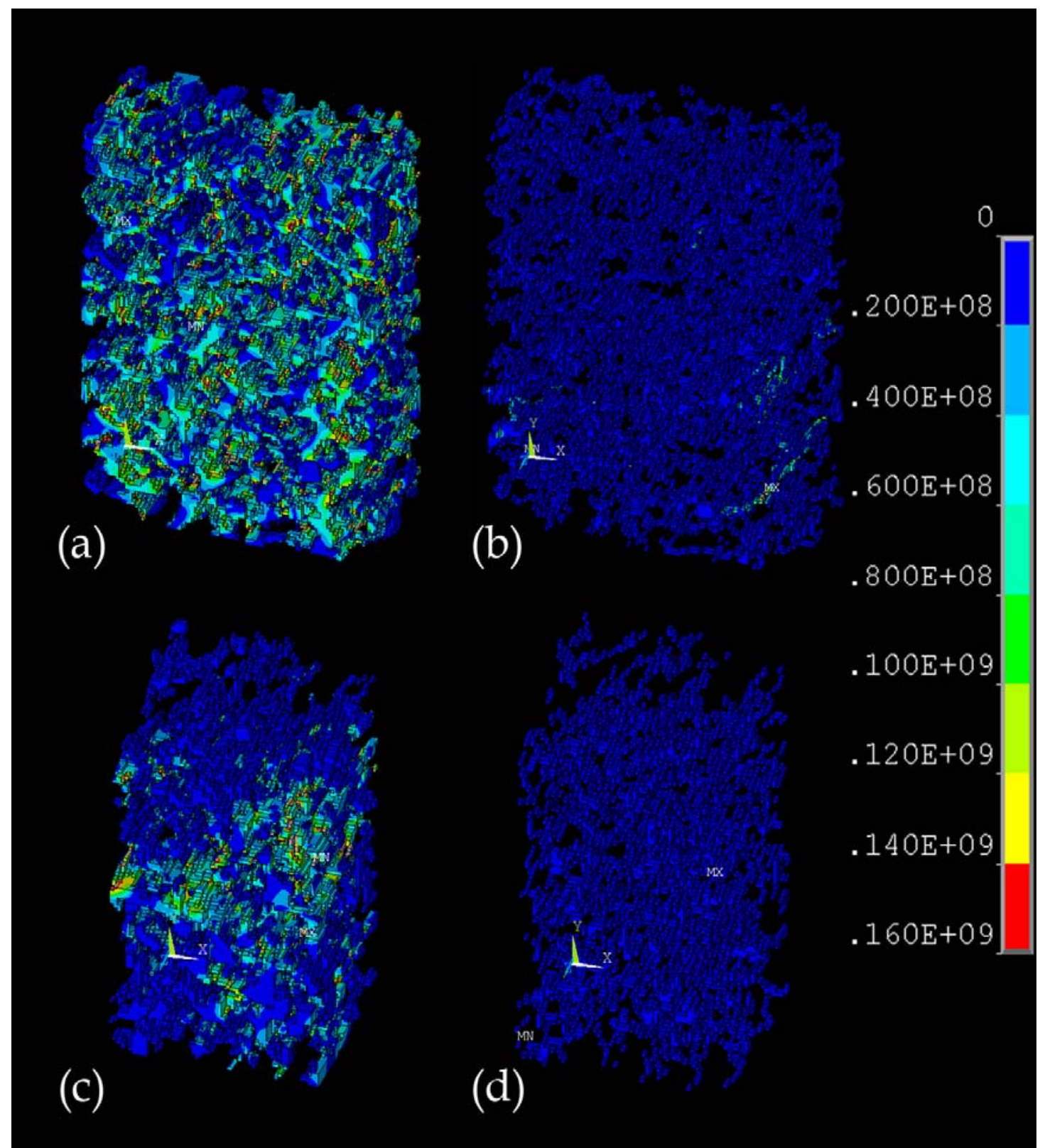

Figure 3.19. Results for the uniaxial compression simulation applied in the z-axis in a case of healthy ( $a-b)$ and osteoporotic (c-d) trabecular bone (stresses in $\mathrm{MPa}$ ). In (a), the parametric stress intensity map for the simulation in a healthy subject can be appreciated, presenting an homogeneous stresses distribution. The stresses results on the corresponding skeletonized structure are depicted in (b). In (c), stress parametric map in a patient with osteoporosis, with heterogeneous stress distribution. The corresponding skeleton with significantly reduced stresses is presented in $(\mathrm{d})$.

\subsubsection{Morphometry and mechanical relationships with $B M D$}

As previously introduced in section 2.7, BMD values from the lumbar spine (L2L4) were available from the patients with osteoporosis due to their routinely clinical workflow. 
The mean $B M D$ value was of $0.73 \pm 0.08 \mathrm{~g} / \mathrm{cm}^{2}$. A significant relationship was found between distal radius $B V / T V$ parameter and spinal $B M D(\mathrm{r}=0.64, \mathrm{p}=0.02)$, explaining a direct association between the bone density values at both locations.

The relationship between fractal dimensions and $B M D$ showed that the $D^{2 D}$ parameter was not significantly related with the spinal $B M D$ measurements $(\mathrm{r}=0.43, \mathrm{p}=0.16)$. However, a modest trend to a relationship was appreciated. The $D^{3 D}$ results also showed no association with the spinal $B M D$ measurements $(\mathrm{r}=0.23, \mathrm{p}=0.48)$, with a lower significance than $D^{2 D}$.

The relationships between $E_{a p p}$ results and $B M D$ were also analyzed. No significant relationships were detected between $E_{a p p}$ and $B M D$ for any of the three compression directions $(\mathrm{r}=0.14, \mathrm{p}=0.91 ; \mathrm{r}=0.39, \mathrm{p}=0.74 ; \mathrm{r}=0.46, \mathrm{p}=0.70$; $E_{\text {app }} X, E_{\text {app }} Y, E_{\text {app }} Z$ vs. $B M D$, respectively).

- Discussion:

Despite of the clear relationship between radius $B V / T V$ and spinal $B M D$, the $\mathrm{D}^{2 \mathrm{D}}$ presented no significant relationship with the spinal $B M D$ measurements of the patients group although a modest trend to the association was observed. The $\mathrm{D}^{3 \mathrm{D}}$ also had no apparent relationship with the BMD measurements, with a lower association with $B M D$ than $D^{2 D}$, which could be preliminarily explained by the different structural information that might provide a 3D approach to the calculation of the fractal dimension compared to $D^{2 D}$ measurements. However, this conclusion should be considered with caution, since the parameters have been measured at different locations. The work developed by Hudelmaier et al. also found that the $D^{2 D}$ parameter was not related to $B M D$ measurements (whereas other parameters like $B V / T V$ or $T b$.Th showed a relationship), and confirmed the independent information provided by $D^{2 D}$ if compared to $B M D$ [120].

$B M D$ is frequently used as one of the parameters for stratifying patients, and it is also used as a gold standard or comparison metric, but it must be noted that 
$B M D$ measures are a measure of projected density, while morphometry and mechanical measures represent 3D properties of trabecular bone. This is demonstrated in the mediocre correlation between $B M D$ and the measures of bone structure and between $B M D$ and the mechanical parameters. Bone turnover affects the architecture, and these changes in cross-sectional measures may not be resolved by projectional DXA measures alone [98].

\subsection{Reproducibility analysis}

The reproducibility of the measurements performed with the developed methodology for spongy bone characterization was assessed following the processes introduced in section 2.6.

The 5 sheep legs were scanned in three consecutive days by the 3T MR system with satisfactory image quality in all the acquisitions. The developed methodology was applied to the images in order to calculate the morphometry and mechanical characteristics of the bone trabeculae.

An initial repeated measures ANOVA was performed in order to evaluate differences between the results calculated in the three datasets. The corresponding $\mathrm{p}$-values from the linear test in the morphometry measurements can be appreciated in table 3.11.

\begin{tabular}{|c|c|c|c|c|c|c|c|c|c|c|}
\hline & BV/TV & Tb.Th & Tb.Sp & Tb.N & D2D & D3D & SCR & TEI & DA & 日a \\
\hline $\mathbf{p}$ & 0.80 & 0.60 & 0.28 & 0.86 & 0.54 & 0.60 & 0.56 & 0.51 & 0.69 & 0.85 \\
\hline
\end{tabular}

Table 3.11. Calculated p-values from the application of the repeated measures ANOVA test in order to evaluate the differences in the morphometry parameters quantified from the three different acquisitions.

Analogously, the p-values for the Young's modulus measurements may be summarized in table 3.12 . 


\begin{tabular}{|c|c|c|c|}
\hline & $\mathbf{E}_{\text {app }} \mathbf{X}$ & $\mathbf{E}_{\text {app }} \mathbf{Y}$ & $\mathbf{E}_{\text {app }} \mathbf{Z}$ \\
\hline $\mathbf{p}$ & 0.29 & 0.36 & 0.41 \\
\hline
\end{tabular}

Table 3.12. Calculated p-values from the application of the repeated measures ANOVA test in order to evaluate the differences in the mechanical parameters quantified from the three different acquisitions.

- Discussion:

As it can be concluded, no significant differences existed for the measurements performed in the three different acquisitions with the methodology exposed in the present thesis. This result is of high relevancy for the consideration of these parameters as disease biomarkers.

Once it has been proved that the measures do not present significant variations between different acquisitions, the error or imprecision of the methods was calculated.

The percentages of change obtained for the morphometry parameters are appreciated in table 3.13 .

\begin{tabular}{|c|c|c|c|c|c|c|c|c|c|c|}
\hline & BVTV & TbTh & TbSp & TbN & D $^{2 D}$ & $D^{3 D}$ & TEI & SCR & DA & $\theta_{\mathrm{a}}$ \\
\hline $\begin{array}{r}\text { RMS } \\
\text { CoV } \\
{[\%]}\end{array}$ & 6.1 & 1.3 & 4.6 & 5.1 & 2.2 & 1.8 & 2.5 & 2.4 & 3.6 & 3.6 \\
\hline
\end{tabular}

Table 3.13. Variability of the morphometry measurements considering the 5 sheep tibiae acquisitions in 3 consecutive days.

The variability of the morphology parameters was very good (approximately between $1 \%$ to $6 \%$ ), with $T b$.Th being the most reproducible parameter. The results obtained for $D^{2 D}$ and $D^{3 D}$ were also highly reproducible, with more reproducibility for the 3D approach for the calculation of the fractal dimension. These results are in accordance with the observed in figure 3.14, where a more concentrated distribution of the results is obtained for $D^{3 D}$ in comparison to $D^{2 D}$. Topology and anisotropy parameters show also a reduced variability. These results are in accordance with the reproducibility analysis of morphometry parameters performed by Gomberg et al. [126]. 
The percentages of change obtained for the mechanical parameters are appreciated in table 3.14 .

\begin{tabular}{|l|c|c|c|}
\hline & $E_{\text {app }} X$ & $E_{\text {app }} Y$ & $E_{\text {app }} Z$ \\
\hline RMS_CoV [\%] & 8.01 & 8.64 & 8.31 \\
\hline
\end{tabular}

Table 3.14. Variability of the $E_{\text {app }}$ calculated in the three space compression directions considering the 5 sheep tibiae acquisitions in 3 consecutive days.

As appreciated, a very good reproducibility was also obtained for the Young's modulus results (between 8 to $9 \%$ ).

The overall results of the reproducibility analysis confirm the exposed methodology to be robust for trabecular bone characterization, with low differences between longitudinal studies.

\subsection{Validation of the measurements}

In order to evaluate the accuracy of the cancellous bone microarchitecture measurements performed by high spatial resolution MRI, a validation test to compare the MR-derived measurements with a technique working at a higher spatial resolution like $\mu \mathrm{CT}$ was designed. The details are emphasized in section 2.6 .

The 5 trabecular bone samples of the sheep tibiae were efficiently scanned by $\mu \mathrm{CT}$. Representative slices from the acquisitions can be appreciated in figure 3.20 .

The results obtained for MR-derived parameters were compared to the obtained using $\mu \mathrm{CT}$. In table 3.15 , the mean values of the morphometry measures performed using MRI and $\mu \mathrm{CT}$ are summarized including the corresponding $\mathrm{p}$ values of the Student's t-test. 


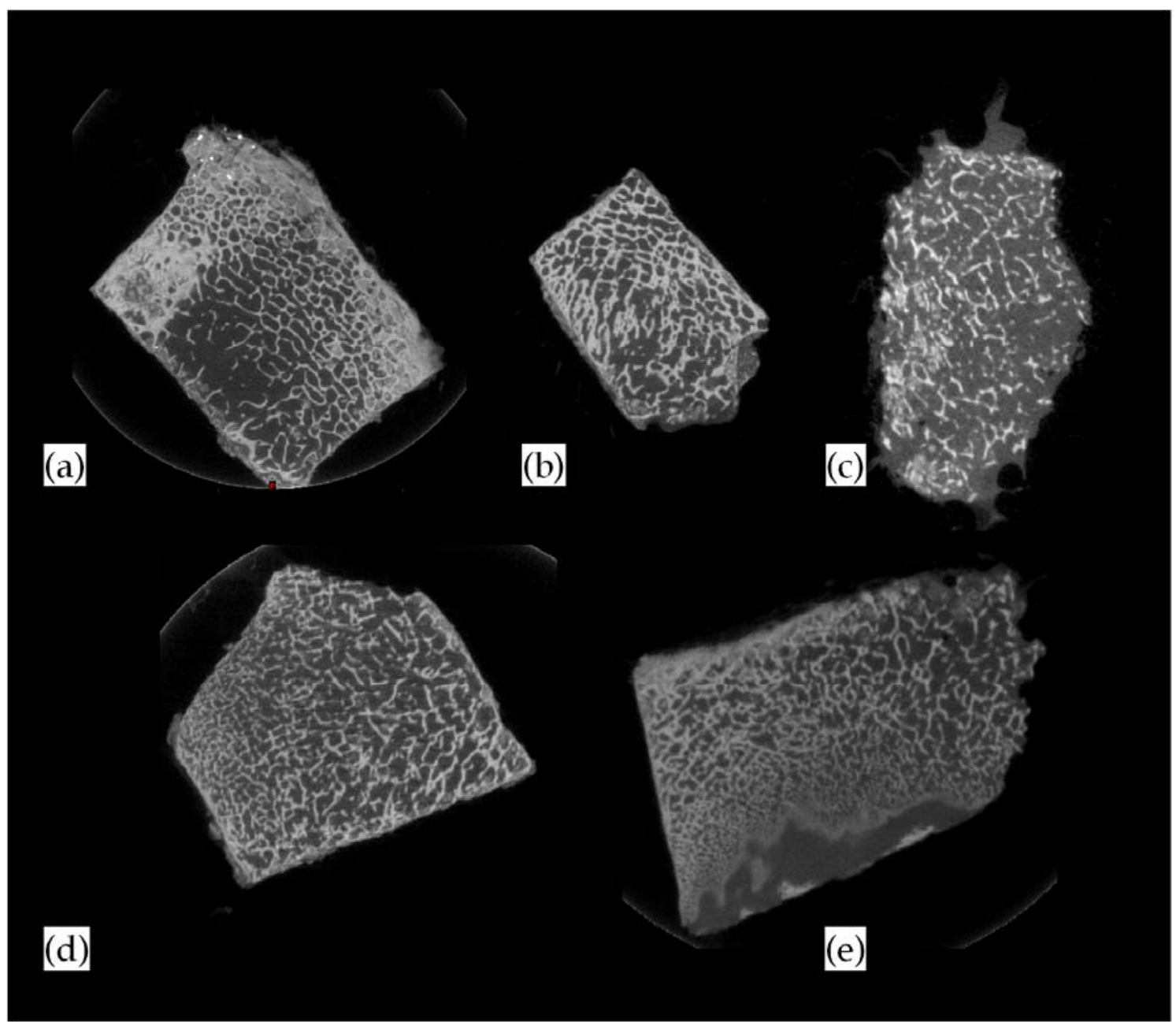

Figure 3.20. Representative images of the 5 different slices extracted from the $\mu \mathrm{CT}$ acquisitions performed in the samples of sheep tibiae, in (a) to (e).

\begin{tabular}{|c|c|c|c|c|c|c|c|c|c|c|}
\hline & $\begin{array}{c}\text { BV/TV } \\
{[\%]}\end{array}$ & $\begin{array}{c}\text { Tb.Th } \\
{[\mathbf{m m}]}\end{array}$ & $\begin{array}{c}\text { Tb.Sp } \\
{[\mathbf{m m}]}\end{array}$ & $\begin{array}{c}\text { Tb.N } \\
{\left[\mathbf{m m}^{-1}\right]}\end{array}$ & $\mathbf{D}^{2 \mathbf{D}}$ & $\mathbf{D}^{3 \mathbf{D}}$ & SCR & TEI & DA & $\boldsymbol{\theta}_{\mathbf{a}}$ \\
\hline $\mathbf{M R}$ & 0.29 & 0.19 & 0.73 & 1.52 & 1.69 & 2.33 & 0.43 & 12.5 & 1.20 & $61^{\mathrm{o}}$ \\
\hline $\boldsymbol{\mu C T}$ & 0.30 & 0.13 & 0.53 & 2.42 & 1.69 & 2.44 & 0.55 & 9.9 & 1.39 & $56^{\mathrm{o}}$ \\
\hline $\mathbf{p}$ & 0.83 & 0.001 & 0.02 & 0.01 & 0.91 & 0.20 & 0.18 & 0.12 & 0.14 & 0.20 \\
\hline $\mathbf{v a l u e}$ & & & & & & & & & & \\
\hline
\end{tabular}

Table 3.15. Morphometry parameters calculated from the 5 sheep tibiae using both MR and $\mu \mathrm{CT}$ techniques. The corresponding $\mathrm{p}$-values showing the significances of differences are also shown. 
In table 3.16, the mean values of the $E_{a p p}$ measures performed using MRI and $\mu \mathrm{CT}$ and the corresponding p-values of the differences are summarized.

\begin{tabular}{|c|c|c|c|}
\hline & $\begin{array}{c}\mathbf{E}_{\text {app }} \mathbf{X} \\
{[\mathbf{M P a}]}\end{array}$ & $\begin{array}{c}\text { Eapp } \mathbf{Y} \\
{[\mathbf{M P a}]}\end{array}$ & $\begin{array}{c}\mathbf{E}_{\mathrm{app}} \mathbf{Z} \\
{[\mathbf{M P a}]}\end{array}$ \\
\hline $\mathbf{M R}$ & 244.68 & 283.94 & 347.74 \\
\hline $\boldsymbol{\mu C T}$ & 269.70 & 307.77 & 408.97 \\
\hline p-value & 0.94 & 0.84 & 0.70 \\
\hline
\end{tabular}

Table 3.16. Mechanical parameters calculated from the 5 sheep tibiae using both MR and $\mu \mathrm{CT}$ techniques. The corresponding $\mathrm{p}$-values showing the significances of differences are also shown.

- Discussion:

As it can be appreciated, significant differences exist for the Tb.Th, Tb.Sp and Tb.N morphology parameters. Such differences are mainly due to partial volume effects of MR. The fractal, topology and anisotropy parameters showed no significant differences between both methodologies. The development which is experiencing MR technology will lead to the increase in the spatial resolutions used in acquisition and may improve the future morphometry measurements. Also, recent upsampling techniques applied to MR data will improve the accuracy of MR-derived parameters for the characterization of cancellous bone tissue [127].

From these results it can be proved that Young's modulus obtained values from MR acquisitions are close to those calculated from the $\mu \mathrm{CT}$ examinations. The stress-strain relationships tend to behave in a similar way in the structures simulated from both MR and $\mu \mathrm{CT}$ data. These results are similar to the obtained in a similar study, where stress distribution between MR-derived and $\mu \mathrm{CT}$ FE models was of the same order whereas MDCT-derived simulations tended to 
overestimate the trabecular bone amount and stress due to considerable partial volume effects, as it can be appreciated in figure 3.21 [128].

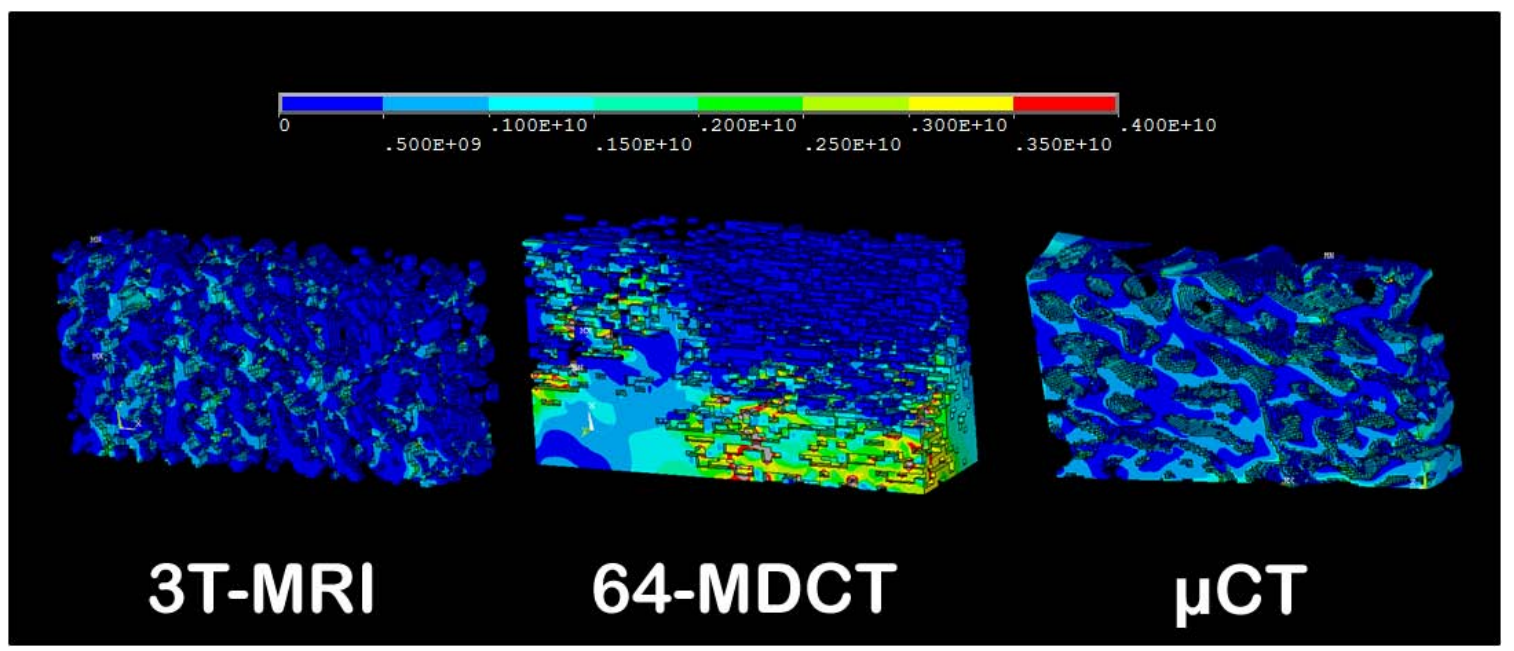

Figure 3.21. Parametric reconstructions of the nodal stress results for the compression simulation using three different modalities (3T-MRI, MDCT and $\mu \mathrm{CT}$ ) [128].

The relative errors of the measures were calculated in order to obtain a percentage of inaccuracy. They can be seen in tables 3.17 and 3.18 for the morphometry and mechanical parameters, respectively.

\begin{tabular}{|c|c|c|c|c|c|c|c|c|c|c|}
\hline & BV/TV & Tb.Th & Tb.Sp & Tb.N & D 2D & D 3D & SCR & TEI & DA & $\boldsymbol{\theta}_{\mathbf{a}}$ \\
\hline $\begin{array}{c}\text { error } \\
{[\%]}\end{array}$ & 3.3 & 46.7 & 37.7 & 37.2 & 0.2 & 4.5 & 21.8 & 26.3 & 13.7 & 8.9 \\
\hline
\end{tabular}

Table 3.17. Relative errors of the morphometry measurements performed using MR considering the $\mu \mathrm{CT}$-derived parameters as the reference.

\begin{tabular}{|c|c|c|c|}
\hline & $\mathbf{E}_{\mathrm{app}} \mathbf{X}$ & $\mathbf{E}_{\mathrm{app}} \mathbf{Y}$ & $\mathbf{E}_{\mathrm{app}} \mathbf{Z}$ \\
\hline error [\%] & 9.3 & 7.7 & 14.9 \\
\hline
\end{tabular}

Table 3.18. Relative errors of the mechanical measurements performed using MR considering the $\mu \mathrm{CT}$-derived values as the reference. 
Finally, it can be concluded that the results obtained with the methodology presented in the current thesis have shown a good accuracy for the trabecular bone microarchitecture characterization, in spite of the differences in morphology parameters, which are a direct consequence of the inherent partial volume effects when studying such small structure from MR images. 


\section{Conclusions}

\section{Conclusions}

In the following it is summarized the main conclusions obtained throughout this thesis:

- The different methodological developments and clinical applications presented in this thesis have demonstrated the feasibility of the morphometry and mechanical analysis of trabecular bone from in vivo MR images as a relevant medical aid for the study of osteoporosis disease.

- An optimized MR acquisition protocol was configured using a 3 Tesla system in order to obtain a high spatial resolution in isotropic voxels with satisfactory signal-to-noise ratio and contrast. This was the first reported MR clinical protocol at such high spatial resolutions.

- The methodological developments for images preparation were fully performed in 3D in order to take advantage of the high spatial resolution of the data. Initial automated and semi-automated segmentation methods were developed to extract trabecular bone tissue. An algorithm for coil shading correction based on 3D calculation of the local intensities was developed. A sub-voxel processing algorithm was implemented in order to increase spatial resolution. Finally, a thresholding algorithm was implemented in 3D to binarize the images and classify the voxels in bone or marrow.

- New methodology was developed for the morphometric characterization of cancellous bone. The morphometric quantification comprised the development of algorithms for the calculation of bone morphology, complexity, topology and anisotropy. As original contributions, new methods for the calculation of the fractal dimension in $3 \mathrm{D}$, and also the tensor scale in 3D for anisotropy analysis were developed. 
- A set of algorithms for an integrated mechanical analysis of the cancellous bone were also developed. First, an efficient new algorithm for generation of voxel-based finite element structures was developed. Also, different routines for the generation of the finite element models in ANSYS format and perform the finite element simulations in batch mode were developed. Finally, algorithms for an automated calculation of the Young's modulus in the three space directions using homogenization theory were implemented.

- The methodology was applied to a series of healthy subjects in order to obtain normality ranks for the morphometry and mechanical parameters calculated. Morphology, topology and mechanical parameters were sensitive to trabecular bone differences between males and females. In general, age was not related to morphometry nor mechanical results.

- In order to evaluate the parameters variations in osteoporosis conditions, the developed methodology was applied to a group of post-menopausal female patients and compared to the values observed in an age-matched healthy population. Morphometry and mechanical parameters showed a high sensitivity to the disease, with significant differences between healthy and osteoporosis values. Morphology parameters showed differences between both groups, although with no high significances. To be noted, the development of 3D methods for fractal characterization improved the results from the previous 2D methodology. The topology results showed differences in bone curves and surfaces between groups. The anisotropy analysis showed an increase of the degree of anisotropy under osteoporosis conditions, which confirmed existing theories. Finally, the mechanical analysis provided significant differences between groups for longitudinal Young's modulus and one of the calculated in the transverse directions.

- The reproducibility of the methodology was evaluated by the repeated MR acquisitions and post-processing of the cancellous bone from 5 sheep 
tibiae in three consecutive days. All the parameters showed a high reproducibility, since no significant differences between the repetitions existed and had percentages of change under $9 \%$.

- Finally, the accuracy of the methodology was evaluated by the comparison of the MR-derived results from the sheep trabecular bone with the values obtained using $\mu \mathrm{CT}$ technique, which worked at a higher spatial resolution. Despite the morphology parameters showed differences due to the partial volume effects of MR acquisitions, the rest of the morphometry and mechanical parameters were close to the obtained from $\mu \mathrm{CT}$.

- A software tool for the automated morphometry and mechanical characterization of cancellous bone in clinical routine has been implemented in the Radiology Department at Hospital Quirón Valencia. 


\section{Future work}

The variety of topics that have been addressed in this thesis is large. However, there is still an enormous amount of work that remains to be done in this field. In addition, the results and conclusions obtained throughout this work raise new questions and suggest several interesting lines of research that need to be explored.

Let us point out in this section the most relevant of them.

\subsection{Evaluation of patients with different ethiopathogenesis.}

The patients analyzed in the present study were all post-menopausal female, and their osteoporosis status could be classified as to be moderate or severe. A larger and wider study cohort with different stages of the disease, including additional data like the number of vertebral fractures, would be relevant.

In particular, the evaluation of the methodology in a large group of patients with initial osteopenia would be very relevant for the analysis of the variations of the morphometry and mechanical parameters at the early cancellous bone changes.

Also, the extension of study of the microarchitecture status to male patients with corticoids-induced osteoporosis would add more information regarding the resorptive processes which are activated in such conditions.

Furthermore, more clinical data, including the recent FRAX® tool developed by the WHO [129] would improve our knowledge about the clinical scenario of the disease. 


\subsection{Longitudinal analysis of the morphometry and mechanical parameters in a group of patients under treatment.}

The application of the techniques presented herein to the study of patients under treatment would be crucial for the widespread consideration of the methodology. If 3D imaging methods are capable to detect beneficial bone microarchitecture changes not directly appreciable in BMD differences, a large number of clinical trials could be initiated with the aim of evaluating the most efficient therapy in reduced times. In figure 5.1, the role of imaging biomarkers in different phases of clinical trials can be appreciated [130].

Phases of a clinical trial

(a)

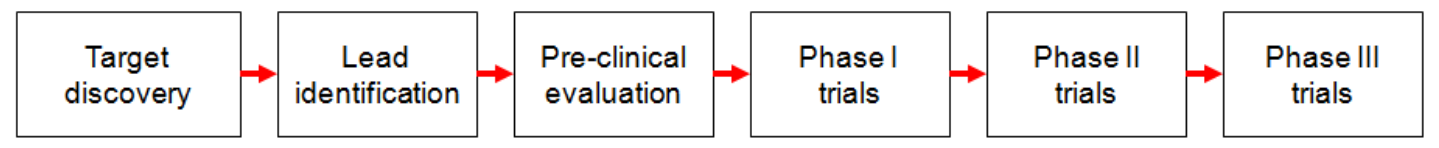

Imaging Biomarkers utility in clinical trials

(b)
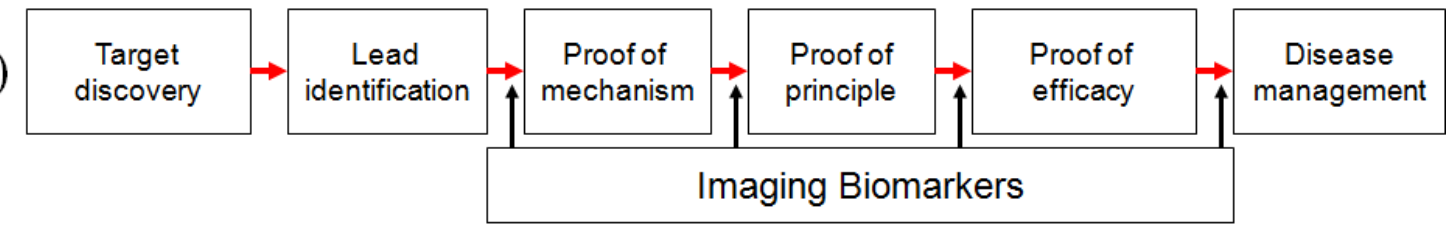

Figure 5.1. In (a), conventional path for drug development. In (b), utility of imaging biomarkers in a proof-of-mechanism, proof-of-principle, and proof-of-efficacy clinical study.

\subsection{Use of MDCT for the trabecular bone characterization.}

Although the applicability of MDCT for the evaluation of microarchitecture characteristics of trabecular bone has been proven to be possible with good results [51], its use is currently limited by the relatively low spatial resolution in comparison to $3 \mathrm{~T}$ MR or HR-pQCT, and also by the radiation dose that is administered to the patient during acquisition.

However, the technology of CT scanners is currently growing and new array of detectors are being developed. Also, more than one X-Ray sources are being 
installed nowadays in a number of CT systems with a significant reduction of acquisition times. These advances may also improve the spatial resolution in acquisition while decrease the current radiation dose in most examinations.

In addition, the recent progresses in MRI upsampling [127] could be also applied to MDCT in order to increment the spatial resolution and evaluate the robustness of the methods for trabecular bone characterization with MDCT upsampled data.

Finally, the BMD can now easily be measured in MDCT scanners, bone density values could be calculated from many acquisitions where the vertebrae are present, such as routine abdomen examinations in order to collect large amounts of densitometry data an include them in databases of osteoporosis disease for clinical research.

\subsection{Mechanical simulations of trabecular bone in the non-linear regime.}

The $\mu \mathrm{FE}$ models defined for the simulations in the present thesis were characterized by linear, elastic and isotropic properties of the bulk material forming the elements. As a consequence, the mechanical simulations of compression were performed in the linear region of the stress-strain curve. However, there is still an interesting part of the curve which is based on the non-linear deformations and ends in the fracture limit.

An iconography of the stress-strain relationship is shown in figure 5.2, where the different elastic and plastic regions can be differentiated. 


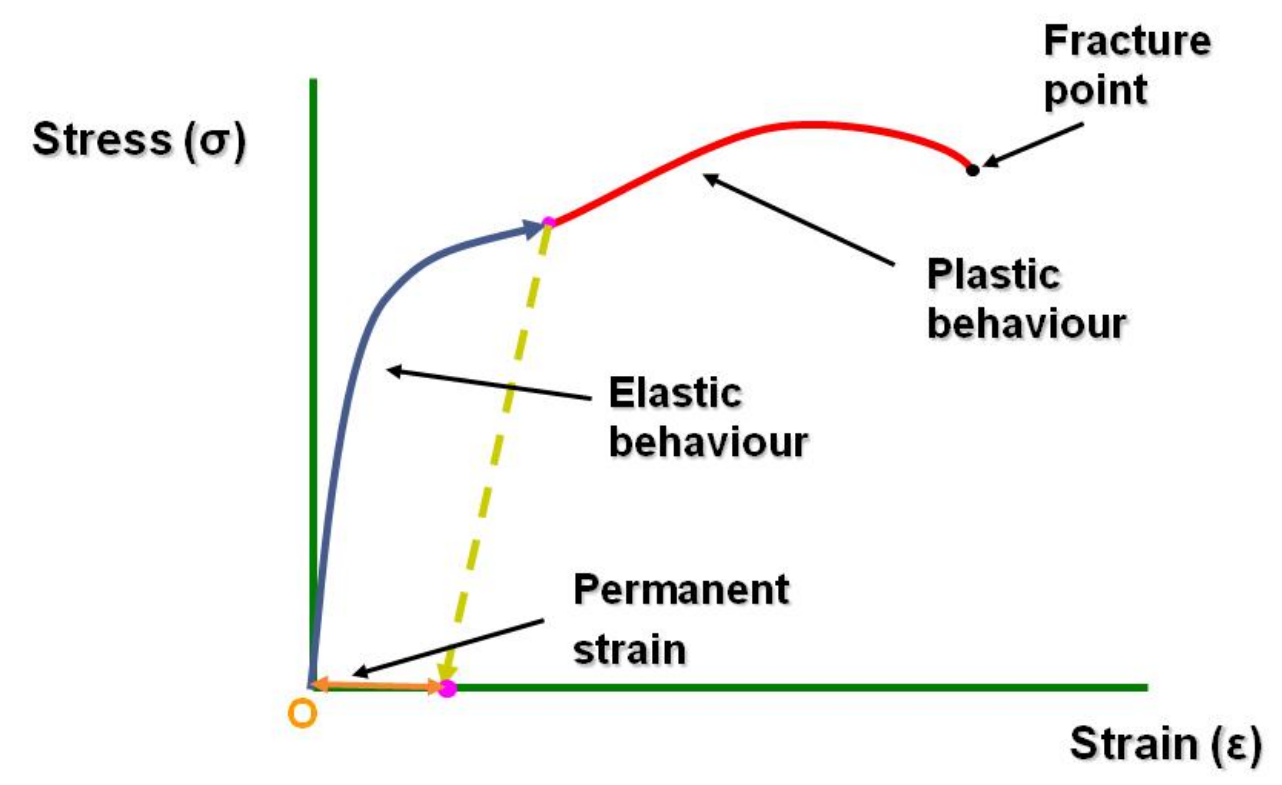

Figure 5.2. The elastic behavior takes place in the linear region of the stress-strain curve. The non-linear deformations region of the curve represents the non-recoverable strain produced in a given material and ends in the fracture point.

A proper and more exact evaluation of the fracture risk should be performed by the analysis of the non-linear stress-strain part of the curve. However, the computational burden due to high complexity of equations may limit the applicability in the clinical routine due to the long computation times.

\subsection{Study of cortical bone water using ultra-short echo times.}

Cortical bone is compound approximately in $20 \%$ water by volume [131]. The microscopic pores of the haversian and the lacunocanalicular systems contain the major part of it. A smaller fraction of cortical bone water is bound to collagen and the matrix substrate, and some tightly bound water is imbedded in the crystals of the apatite like mineral. The hydrated state of bone is essential in conferring bone viscoelastic properties [132].

MRI offers a promising technique for the quantification of bone water, even though this is not possible with conventional imaging techniques as they are currently used for soft-tissue visualization. 
The extremely short transverse relaxation times $(\mathrm{T} 2<500 \mu \mathrm{s})$ of pore water protons result from surface interactions and diamagnetism of the mineral relative to water. Consequently, the bone water signal is not detectable with standard imaging pulse sequences in which echo times typically are on the order of milliseconds. However, bone water can be depicted in vivo with suitable MR imaging techniques, which employ radial readouts in conjunction with short-duration radiofrequency pulses to enable the acquisition to start tens of microseconds after excitation [133].

Some results in specimens and in live human subjects suggest that ultrashort echo time MR imaging may allow routine quantification of cortical bone porosity as a new metric of bone quality in patients with metabolic bone disease [134].

In figure 5.3, an image of the wrist acquired with a MR sequence using ultrashort echo times can be appreciated. Water contained in the cortical bone pores produce a slightly bright signal in the images.

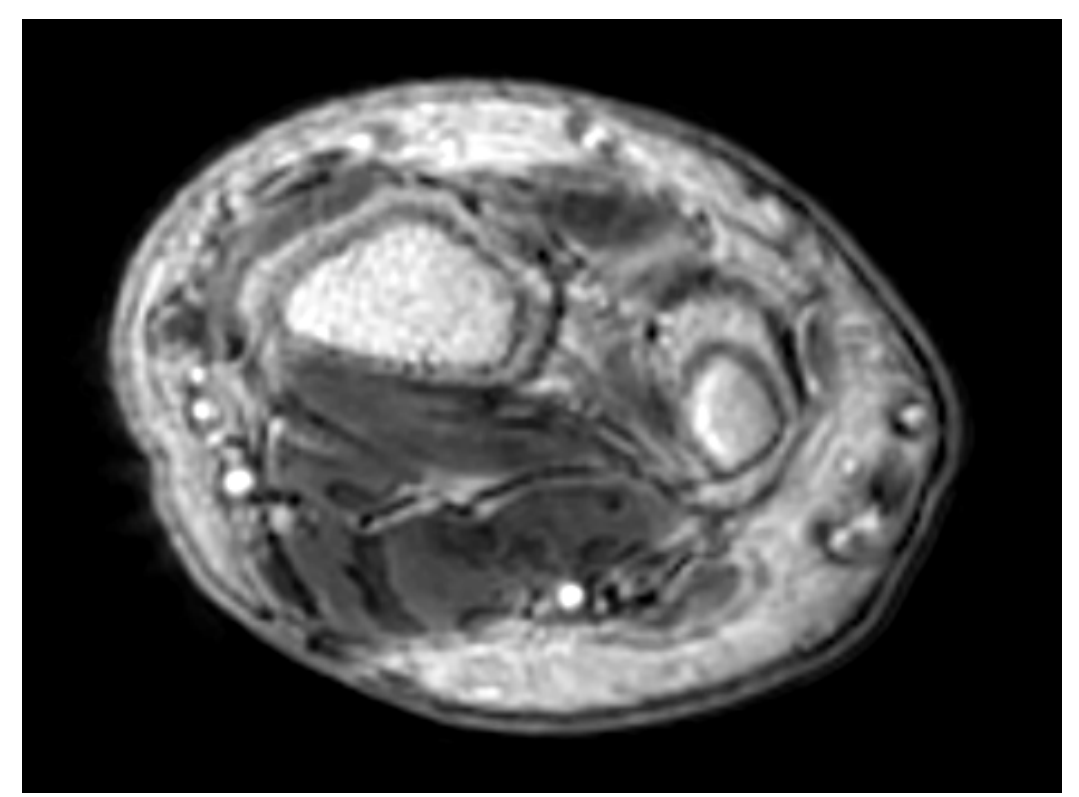

Figure 5.3. Axial image of a wrist using an MR sequence with ultrashort echo time. The cortical bone of the radius is well depicted and shows a slightly bright signal since the relaxation of water protons within the cortical bone pores has been captured. 


\subsection{Imaging based evaluation of bone deposition on polymer scaffolds in tissue engineering.}

The methodology exposed in the current thesis could be further applied for the quantification of bone deposition on the inner surfaces of polymer tissue engineered scaffolds.

The evaluation of the functionality of the engineered scaffolds is an important issue in the evaluation of the scaffolds synthesis processes. The use of image processing techniques oriented to small structure analysis from morphometry and mechanical characterizations would improve the evaluation of the fabric designed as a substrate for the bone formation.

\subsection{Development of an integrated image post-processing platform.}

The software application developed in this thesis has been implemented in Matlab as a well structured prototype. It has also the possibility of generating a clinical report containing the morphometry and mechanical results.

Some of the algorithms that form the application are also common in other medical imaging applications, like interpolation or thresholding. The algorithms developed for the morphometry and mechanical characterization of trabecular bone could be perfectly integrated in a post-processing platform of imaging biomarkers with a common kernel consisting in DICOM readers and shared pre-processing routines. Final results should be stored in a database for scientific research and automated clinical report generation.

An example of the architecture definition for a medical image post-processing platform can be observed in figure 5.4. 


\section{Future work}

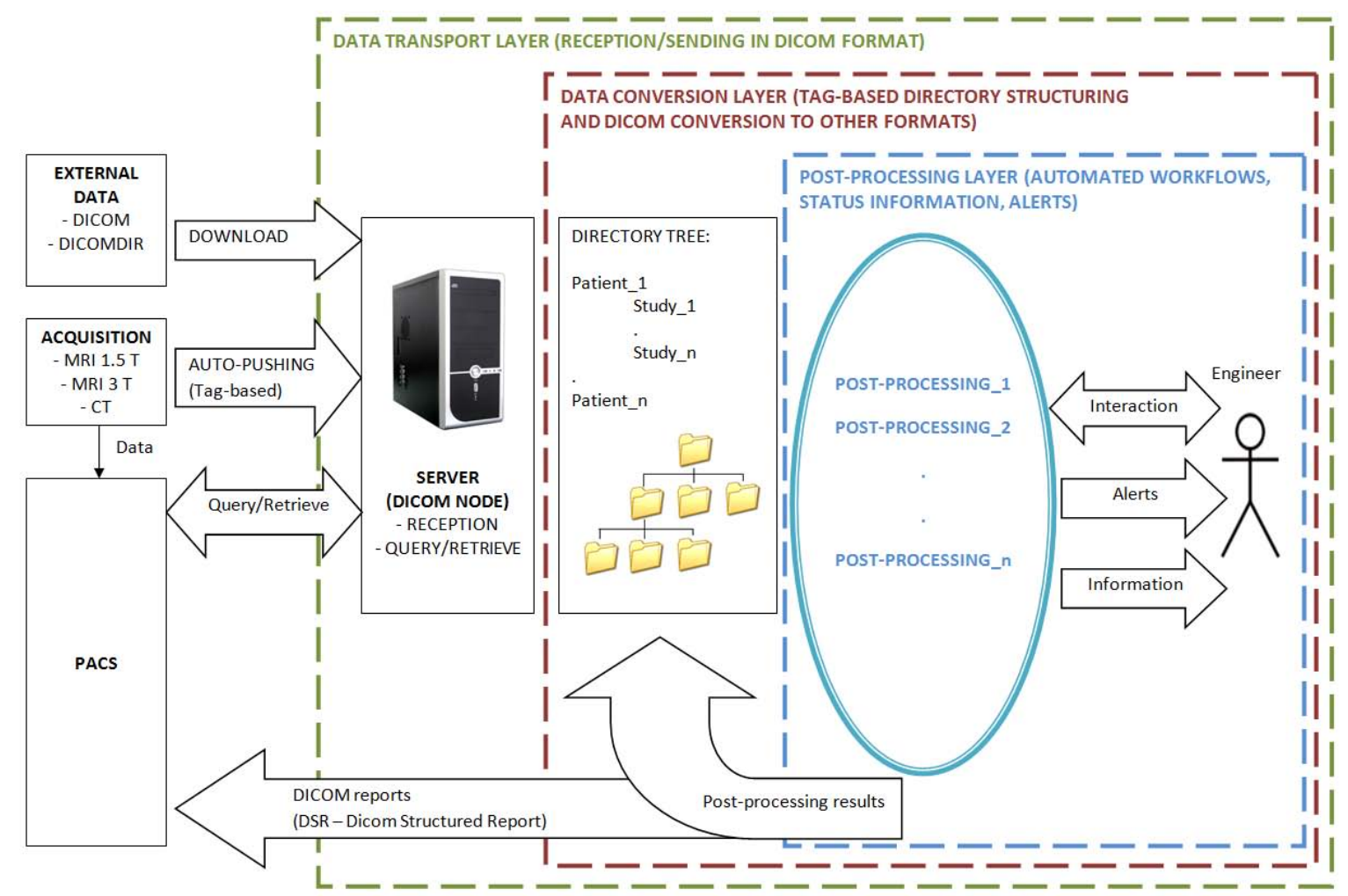

Figure 5.4. Workflow architecture of a medical image post-processing platform for Imaging Biomarkers. The structure is organized mainly in three different layers (data transport, data conversion-preparation and data post-processing). 


\section{References}

[1] Reginster JY, Burlet N. Osteoporosis: a still increasing prevalence. Bone 2006;38:S4-S9.

[2] NIH Consensus Development Panel on Osteoporosis Prevention. Diagnosis and therapy. Osteoporosis, prevention, diagnosis, and therapy. JAMA: the journal of the American Medical Association 2001;285:785-795.

[3] Seeman E. Invited Review: Pathogenesis of osteoporosis. Journal of applied physiology 2003;95:2142-2151.

[4] Wehrli FW. Structural and functional assessment of trabecular and cortical bone by micro magnetic resonance imaging. Journal of Magnetic Resonance Imaging 2007;25:390-409.

[5] Carballido-Gamio J, Majumdar S. Clinical utility of microarchitecture measurements of trabecular bone. Current osteoporosis reports 2006;4:64-70.

[6] Feldkamp LA, Goldstein SA, Parfitt AM, Jesion G, Kleerekoper M. The direct examination of three-dimensional bone architecture in vitro by computed tomography. Journal of Bone and Mineral Research 1989;4:3-11.

[7] Kuhn JL, Goldstein SA, Feldkamp LA, Goulet RW, Jesion G. Evaluation of a microcomputed tomography system to study trabecular bone structure. Journal of Orthopaedic Research 1990;8:833-842.

[8] Hildebrand T, Laib A, Muller R, Dequeker J, Ruegsegger P. Direct threedimensional morphometric analysis of human cancellous bone: microstructural data from spine, femur, iliac crest, and calcaneus. Journal of Bone and Mineral Research 1999;14:1167-1174.

[9] A Textbook of Histology (12 ${ }^{\text {th }}$ edition). Fawcett DW (ed). New York, NY: Chapman \& Hall, 1994.

[10] Servier Medical Art (http:// www.servier.com/). Last access: February 23, 2010 .

[11] Radiology of Osteoporosis. Grampp S (ed). New York, NY: SpringerVerlag, 2002.

[12] Baron R. Anatomy and ultrastructure of bone. In: Favus MJ (ed) Primer on the metabolic bone diseases and disorders of mineral metabolism. New York, NY: Raven, 2003. 
[13] Marks SC, Hermey DC. The structure and development of bone. In: Principles of bone biology. Bilezikian JB, Raize LG, Rodan GA (eds). New York, NY: Academic Press, 1996.

[14] Frost HM. Dynamic of bone remodeling. In: Bone biodynamics. Boston, MA: Little Brown Co, 1964.

[15] Osteoporosis and Bone Physiology. University of Washington (http://courses.washington.edu/bonephys/). Last access: June 15, 2010.

[16] Manolagas SC. Cell number versus cell vigor - what really matters to a regenerating skeleton? Endocrinology 1999;140:4377-4381.

[17] Parfitt AM. The mechanisms of coupling: a role for vasculature. Bone 2000;26:319-323.

[18] Manolagas SC, Jilka RL. Bone marrow, cytokines, and bone remodeling. Emerging insights into the pathophysiology of osteoporosis. New England Journal of Medicine 1995;332:305-311.

[19] Suda T, Takahashi N, Martin TJ. Modulation of osteoclast differentiation. Endocrine Reviews 1992;13:66-80.

[20] Hollinger JO, Buck DC, Bruder SB. Biology of bone healing: its impact on clinical therapy. In: Lynch SE, Genco RJ, Marx RE (eds) Tissue engineering. Carol Stream, USA: Quintessence Publishing Co, 1999.

[21] International Osteoporosis Foundation (http://www.iofbonehealth.org). Last access: May 23, 2010.

[22] Kanis JA, Johnell O. Requirements for DXA for the management of osteoporosis in Europe. Osteoporosis International 2004;16:229-238.

[23] Sanfélix-Genovés J, Reig-Molla B, Sanfélix-Gimeno G, Peiró S, GraellsFerrer M, Vega-Martínez $M$, et al. The population-based prevalence of osteoporotic vertebral fracture and densitometric osteoporosis in postmenopausal women over 50 in Valencia, Spain (the FRAVO Study). Bone 2010;17. [Epub ahead of print].

[24] Gullberg B, Johnell O, Kanis JA. World-wide projections for hip fracture. Osteoporosis International 1997;7:407-413.

[25] Cummings SR, Melton III JR. Epidemiology and outcomes of osteoporotic fractures. Lancet 2002;359:1761-1767. 
[26] WHO assessment of fracture risk and its application to screening for postmenopausal osteoporosis. WHO technical report series 843, Geneva, Switzerland; 1994.

[27] Duan Y, Turner CH, Kim BT, Seeman E. Sexual dimorphism in vertebral fragility is more the result of gender differences in age-related bone gain than bone loss. Journal of Bone and Mineral Research 2001;16:2267-2275.

[28] Recker RR, Barger-Lux MJ. The elusive concept of bone quality. Current Osteoporosis Reports 2004;2:97-100.

[29] Stokstad E. Bone quality fills holes in fracture risk. Science 2005; 308:15801581.

[30] Rubin CD. Emerging concepts in osteoporosis and bone strength. Current Medical Research and Opinion 2005;21:1049-1056.

[31] Wehrli FW, Saha PK, Gomberg BR, Song HK, Snyder PJ, Benito M, et al. Role of magnetic resonance for assessing structure and function of trabecular bone. Topics in Magnetic Resonance Imaging 2002;13:335-355.

[32] Gili J. Introducción Biofísica a la Resonancia Magnética Aplicada a la Clínica. V(09-1). Barcelona, España: Jaume Gili, 2009.

[33] Stark DD, Bradley Jr WG. Magnetic Resonance Imaging. Volume I. $3^{\text {rd }}$ ed. Missouri, MO: Mosby, 1999.

[34] Vlaardingerbroek MT, den Boer JA. Magnetic Resonance Imaging. Theory and practice. $3^{\text {rd }}$ ed. Berlin, Germany: Springer, 2003.

[35] Pooley Ra. AAPM/RSNA Physics Tutorial for Residents. Radiographics 2005;25:1087-1099.

[36] Salibi N, Brown MA. Clinical MR Spectroscopy. First principles. Toronto, Canada: Wiley-Liss, 1998.

[37] Kuehn B, Stampa B, Heller M, Glueer C. In vivo assessment of trabecular bone structure of the human phalanges using high resolution magnetic resonance imaging. Osteoporosis International 1997;7:291.

[38] Link TM, Bauer J, Kollstedt A, Stumpf I, Hudelmaier M, Settles M, et al. Trabecular bone structure of the distal radius, the calcaneus, and the spine: which site predicts fracture status of the spine best? Investigative Radiology. 2004;39:487-497. 
[39] Link TM, Vieth V, Langenberg R, Meier N, Lotter A, Newitt D, et al. Structure analysis of high resolution magnetic resonance imaging of the proximal femur: in vitro correlation with biomechanical strength and BMD. Calcified Tissues International. 2003;72:156-165.

[40] Majumdar S. Magnetic resonance imaging of trabecular bone structure. Topics in Magnetic Resonance Imaging 2002;13:323-334.

[41] Techawiboonwong A, Song HK, Magland JF, Saha PK, Wehrli FW. Implications of Pulse Sequence in Structural Imaging of Trabecular Bone. Journal of Magnetic Resonance Imaging 2005;22:647-655.

[42] Haacke EM, Brown RW, Thompson MR, Venkatesan R. Magnetic resonance imaging: physical principles and sequence design. New York, NY: John Wiley \& Sons Inc., 1999.

[43] Majumdar S, Newitt D, Jergas M, Gies A, Chiu E, Osman D, et al. Evaluation of technical factors affecting the quantification of trabecular bone structure using magnetic resonance imaging. Bone 1995;17:417-430.

[44] McKay HA, Sievänen H, Petit MA, MacKelvie KJ, Forkheim KM, Whittall $\mathrm{KP}$, et al. Application of magnetic resonance imaging to evaluation of femoral neck structure in growing girls. Journal of Clinical Densitometry 2004;7:161168.

[45] Whitehouse WJ. The quantitative morphology of anisotropic trabecular bone. Journal of Microscopy 1974;101:153-168.

[46] Odgaard A, Andersen K, Melsen F, Gundersen HJ. A direct method for fast three-dimensional serial reconstruction. Journal of Microscopy 1990;159:335342.

[47] Ruëgsegger P, Koller B, Muller R. A microtomographic system for the nondestructive evaluation of bone architecture. Calcified Tissues International 1996;58:24-29.

[48] Laib A, Hauselmann HJ, Rüegsegger P. In vivo high resolution 3D-QCT of the human forearm. Technology and health care 1998;6:329-337.

[49] Muller R, Hildebrand T, Hauselmann HJ, Ruegsegger P. In vivo reproducibility of three-dimensional structural properties of noninvasive bone biopsies using 3D-pQCT. Journal of Bone and Mineral Research 1996;11:17451750. 
[50] Boutroy S, Bouxsein ML, Munoz F, Delmas PD. In vivo assessment of trabecular bone microarchitecture by high-resolution peripheral quantitative computed tomography. Journal of Clinical Endocrinology \& Metabolism 2005;90:6805-6815.

[51] Ito M, Ikeda K, Nishiguchi M, Shindo H, Uetani M, Hosoi T, et al. Multidetector row CT imaging of vertebral microstructure for evaluation of fracture risk. Journal of Bone and Mineral Research 2005;20:1828-1836.

[52] Benito M, Vasilic B, Wehrli FW, Bunker B, Wald MJ, Gomberg B, et al. Effect of testosterone replacement on bone architecture in hypogonadal men. Journal of Bone and Mineral Research 2005;20:1785-1791.

[53] Link TM, Majumdar S, Augat P, Lin JC, Newitt D, Lu Y, et al. In vivo high resolution MRI of the calcaneus: differences in trabecular structure in osteoporotic patients. Journal of Bone and Mineral Research 1998;13:1175-1182.

[54] Link TM, Saborowski, Kisters K, Kempkes M, Kosch M, Newitt D, et al. Changes in calcaneal trabecular bone structure assessed with high-resolution MRI in patients with kidney transplantation. Osteoporosis International 2002;13:119-129.

[55] Majumdar S, Genant HK, Grampp S, Newitt DC, Truong VH, Lin JC, et al. Correlation of trabecular bone structure with age, bone, mineral density, and osteoporotic status: in vivo studies in the distal radius using high-resolution magnetic resonance imaging. Journal of Bone and Mineral Research 1997;12:111-118.

[56] Wehrli FW, Hwang SN, Ma J, Song HK, Ford JC, Haddad JG. Cancellous bone volume and structure in the forearm: noninvasive assessment with MR microimaging and image processing. Radiology 1998;206:347-357.

[57] Wehrli FW, Gomberg BR, Saha PK, Song HK, Hwang SN, Snyder PJ. Digital topological analysis of in vivo magnetic resonance microimages of trabecular bone reveals structural implications of osteoporosis. Journal of Bone and Mineral Research 2001;16:1520-1531.

[58] Krug R, Banerjee S, Han ET, Newitt DC, Link TM, Majumdar S. Feasibility of in vivo structural analysis of high-resolution magnetic resonance images of the proximal femur. Osteoporosis International 2005;16:1307-1314.

[59] Fernandez-Seara MA, Song HK, Wehrli FW. Trabecular bone volume fraction mapping by low-resolution MRI. Magnetic Resonance in Medicine 2001;46:103-113. 
[60] Bouchard LS, Wehrli FW, Chin CL, Warren WS. Structural anisotropy and internal magnetic fields in trabecular bone: coupling solution and solid dipolar interactions. Journal of Magnetic Resonance 2005;176:27-36.

[61] Feltrin GP, Macchi V, Saccavini C, Tosi E, Dus C, Fassina A, et al. Fractal analysis of lumbar vertebral cancellous bone architecture. Clinical Anatomy 2001;14:414-417.

[62] Mandelbrot BB. The Fractal Geometry of Nature. New York, NY: W.H. Freeman and Company, 1982.

[63] Chappard D, Legrand E, Haettich B, Chalès G, Auvinet B, Eschard JP, et al. Fractal dimension of trabecular bone: comparison of three histomorphometric computed techniques for measuring the architectural two-dimensional complexity. Journal of Pathology 2001;195:515-521.

[64] Majumdar S, Lin J, Link T, Millard J, Augat P, Ouyang X, et al. Fractal analysis of radiographs: assessment of trabecular bone structure and prediction of elastic modulus and strength. Medical Physics 1999;26:1330-1340.

[65] Follet H, Bruyère-Garnier K, Peyrin F, Roux JP, Arlot ME, Burt-Pichat B, et al. Relationship between compressive properties of human os calcis cancellous bone and microarchitecture assessed from 2D and 3D synchrotron microtomography. Bone 2005;36:340-351.

[66] Alberich-Bayarri A, Marti-Bonmati L, Sanz-Requena R, Belloch E, Moratal D. In vivo trabecular bone morphologic and mechanical relationship using high-resolution 3-T MRI. American Journal of Roentgenology 2008;191:721-726.

[67] Parfitt AM, Mathews CHE, Villanueva AR, Kleerekoper M, Frame B, Rao DS. Relationships between surface, volume, and thickness of iliac trabecular bone in aging and in osteoporosis. Implications for the microanatomic and cellular mechanisms of bone loss. The Journal of Clinical Investigation 1983;72:1396-1409.

[68] Kinney JH, Ladd AJC. The relationship between three-dimensional connectivity and the elastic properties of trabecular bone. Journal of Bone and Mineral Research 1998;13:839-845.

[69] Saha PK, Chaudhuri BB. 3D digital topology under binary transformation with applications. Computer Vision and Image Understanding 1996;63:418-429.

[70] Gomberg BR, Saha PK, Song HK, Hwang SN, Wehrli FW. Topological analysis of trabecular bone MR images. IEEE Transactions on Medical Imaging 2000;19:166-174. 
[71] Wolff J. Das Gesetz der Transformation der Knochen. Berlin, DE: Hirschwald, 1892.

[72] Saha PK, Wehrli FW. A robust method for measuring trabecular bone orientation anisotropy at in vivo resolution using tensor scale. Pattern Recognition 2004;37:1935-1944.

[73] Chevalier Y, Pahr D, Allmer H, Charlebois M, Zysset P. Validation of a voxel-based FE method for prediction of the uniaxial apparent modulus of human trabecular bone using macroscopic mechanical tests and nanoindentation. Journal of Biomechanics 2007;40:3333-3340.

[74] Pruessmann KP, Weiger M, Scheidegger MB, Boesiger P. SENSE: Sensitivity encoding for fast MRI. Magnetic Resonance in Medicine 1999;42:952962.

[75] Banerjee S, Choudhury S, Han ET, Brau AC, Morze CV, Vigneron DB, et al. Autocalibrating parallel imaging of in vivo trabecular bone microarchitecture at 3 Tesla. Magnetic Resonance in Medicine 2006;56:1075-1084.

[76] Nieto-Charques L. EsTra: Análisis de la Estructura Trabecular. Herramienta de ayuda al diagnóstico de enfermedades óseas basada en un análisis estructural tridimensional a partir de imágenes por resonancia magnética. Master in Science Project. Directors: Dr. David Moratal, Dr. Luis Marti-Bonmati. Universitat Politècnica de València. July 2007.

[77] Kass M, Witkin A, Terzopoulos D. Snakes: active contours models. International Journal of Computer Vision 1987;1:321-331.

[78] Vasilic B, Wehrli FW. A novel local thresholding algorithm for trabecular bone volume fraction mapping in the limited spatial resolution regime of In vivo MRI. IEEE Transactions on Medical Imaging 2005;24:1574-1585.

[79] Hwang SN, Wehrli FW. Subvoxel processing: a method for reducing partial volume blurring with application to in vivo MR images of trabecular bone. Magnetic Resonance in Medicine 2002;47:948-957.

[80] Otsu N. A threshold selection method from gray-level histogram. IEEE Transactions Systems, Man and Cybernet 1979;9:82-86.

[81] Delaunay B. Sur la sphere vide. A la mémoire de Georges Voronoi. Izvestia Akademii Nauk SSSR, Otdelenie Matematicheskikh i Estestvennykh Nauk. Bulletin of Academy of Sciences of the USSR 1934;7:793-800. 
[82] Rapid prototyping and manufacturing. Materialise. Leuven, Belgium. (http://www.materialise.com/rapid-prototyping). Last access: July 29, 2010.

[83] Laib A, Newitt DC, Lu Y, Majumdar S. New model-independent measures of trabecular bone structure applied to in vivo high resolution MR images. Osteoporosis International 2002;13:130-136.

[84] Saha PK, Wehrli FW. Measurement of Trabecular Bone Thickness in the Limited Resolution Regime of In Vivo MRI by Fuzzy Distance Transform. IEEE Transactions on Medical Imaging 2004;23:53-62.

[85] Tamada T, Sone T, Jo Y, Imai S, Kajihara Y, Fukunaga M. Threedimensional trabecular bone architecture of the lumbar spine in bone metastasis from prostate cancer: comparison with degenerative sclerosis. Skeletal Radiology 2005;34:149-155.

[86] Lorensen WE, Cline HE. Marching cubes: A high resolution 3D surface construction algorithm. Computer Graphics 1987;21:7-12.

[87] Saha PK, Chaudhuri BB, Majumder DD. A new shape preserving parallel thinning algorithm for 3D digital images. Pattern Recognition 1997;30:19391955.

[88] Saha PK, Gomberg RB, Wehrli FW. Three-dimensional digital topological characterization of cancellous bone architecture. International Journal of Imaging Systems and Technology 2000;11:81-90.

[89] Wald MJ, Vasilic B, Saha PK, Wehrli FW. Spatial autocorrelation and mean intercept length analysis of trabecular bone anisotropy applied to in vivo magnetic resonance imaging. Medical Physics 2007;34:1110-1120.

[90] Inglis D, Pietruszczak S. Characterization of anisotropy in porous media by means of linear intercept measurements. International Journal of Solids and Structures 2003;40:1243-1264.

[91] Saha PK. Tensor scale: A local morphometric parameter with applications to computer vision and image processing. Computer Vision and Image Understanding 2005;99:383-413.

[92] López Orive, JJ. Análisis mediante tratamiento de imagen de la anisotropía y de la orientación principal de la estructura trabecular para una ayuda al diagnóstico de la osteoporosis. Aplicación a los andamiajes sustitutivos de biopolímero de hueso trabecular. Master in Science Project. Director: Dr. David Moratal. Universitat Politècnica de València. December 2008. 
[93] Ulrich D, van Rietbergen B, Weinans H, Rüegsegger P. Finite element analysis of trabecular bone structure: a comparison of image-based meshing techniques. Journal of Biomechanics 1998;31:1187-1192.

[94] Apel T, Düvelmeyer N. Transformation of hexaedral finite element meshes into tetrahedral meshes according to quality criteria. Computing 2003;71:293304.

[95] Alberich-Bayarri A, Moratal D, Martí-Bonmatí L, Salmerón-Sánchez M, Vallés-Lluch A, Nieto-Charques L, Rieta JJ. Volume mesh generation and finite element analysis of trabecular bone magnetic resonance images. Conference Proceedings IEEE Engineering in Medicine and Biology Society 2007;2007:16031606.

[96] Ladd AJ, Kinney JH. Numerical errors and uncertainties in finite-element modeling of trabecular bone. Journal of Biomechanics 1998;31:941-945.

[97] Fung YC. Biomechanics. Mechanical properties of living tissues (2nd edition). New York, NY: Springer, 1993.

[98] Newitt DC, Majumdar S, van Rietbergen B, von Ingersleben G, Harris ST, Genant HK, et al. In vivo assessment of architecture and micro-finite element analysis derived indices of mechanical properties of trabecular bone in the radius. Osteoporosis International 2002;13:6-17.

[99] Zienkiewicz OC, Taylor RL, Zhu JZ. The finite element method, its basis \& fundamentals. 6th edition. Oxford: Elsevier, 2006.

[100] Hollister SJ, Fyhrie DP, Jepsen KJ, Goldstein SA. Application of homogenization theory to the study of trabecular bone mechanics. Journal of Biomechanics 1991;24:825-839.

[101] Hollister SJ, Kikuchi N. A comparison of homogenization theory and standard mechanics analyses for periodic porous composites. Computational Mechanics 1992;10:73-95.

[102] Pearce AI, Richards RG, Milz S, Schneider E, Pearce SG. Animal models for implant biomaterial research in bone: a review. European Cells and Materials 2007;13:1-10.

[103] Newman E, Turner AS, Wark JD. The potential of sheep for the study of osteopenia: current status and comparison with other animal models. Bone 1995;16:277S-284S. 
[104] Martini L, Fini M, Giavaresi G, Giardino R. Sheep model in orthopedic research: a literature review. Comparative Medicine 2001;51:292-299.

[105] Sanz R, Martí-Bonmatí L, Rodrigo JL, Moratal D. MR pharmacokinetic modeling of the patellar cartilage differentiates normal from pathological conditions. Journal of Magnetic Resonance Imaging 2008;27:171-177.

[106] Altman DG, Bland JM. Measurement in medicine: the analysis of method comparison studies. Statistician 1983;32:307-317.

[107] Alberich-Bayarri A, Marti-Bonmati L, Pérez MA, Sanz-Requena R, LermaGarrido JJ, García-Martí G, et al. Assessment of 2D and 3D fractal dimension measurements of trabecular bone from high-spatial resolution magnetic resonance images at 3 Tesla. Medical Physics 2010;37:4930-4937.

[108] Pothuaud L, Porion P, Lespessailles E, Benhamou CL, Levitz P. A new method for three-dimensional skeleton graph analysis of porous media: application to trabecular bone microarchitecture. Journal of Microscopy 2000;199:149-161.

[109] Pothuaud L, Laib A, Levitz P, Benhamou CL, Majumdar S. Threedimensional-line skeleton graph analysis of high-resolution magnetic resonance images: a validation study from 34-microm-resolution microcomputed tomography. Journal of Bone and Mineral Research 2002;17:1883-1895.

[110] Ciarelli TE, Fyhrie DP, Schaffler MB, Goldstein SA. Variations in threedimensional cancellous bone architecture of the proximal femur in female hip fractures and in controls. Journal of Bone and Mineral Research 2000;15:32-40.

[111] Geraets WG, Van der Stelt PF, Lips P, Elders PJ, Van Ginkel FC, Burger $\mathrm{EH}$. Orientation of the trabecular pattern of the distal radius around the menopause. J Biomech 1997;30:363-370.

[112] López-Orive JJ, Alberich-Bayarri A, Salmerón M, Martí-Bonmatí L, Moratal D. 3D in-vivo trabecular bone anisotropy and orientation analysis using 3T MR images. 25th Annual Meeting ESMRMB, Valencia, Spain, 2008.

[113] Keaveny TM, Yeh OC. Architecture and trabecular bone: toward an improved understanding of the biomechanical effects of age, sex and osteoporosis. Journal of Musculoskeletal and Neuronal Interactions 2002;2:205208.

[114] Müller R, Rüegsegger P. Three-dimensional finite element modelling of non-invasively assessed trabecular bone structures. Medicine Engeering and Physics 1995;17:126-133. 
[115] Zysset PK. A review of morphology-elasticity relationships in human trabecular bone: theories and experiments. Journal of Biomechanics 2003;36:1469-1485.

[116] Pothuaud L, Van Rietbergen B, Mosekilde L, Beuf O, Levitz P, Benhamou $\mathrm{CL}$, et al. Combination of topological parameters and bone volume fraction better predicts the mechanical properties of trabecular bone. Journal of Biomechanics 2002;35:1091-1099.

[117] Diego RB, Estellés JM, Sanz JA, García-Aznar JM, Sánchez MS. Polymer scaffolds with interconnected spherical pores and controlled architecture for tissue engineering: fabrication, mechanical properties and finite element modeling. J Biomed Mater Res Part B: Appl Biomater 2007;81B:448-455.

[118] Gibson LJ, Ashby M. The mechanics of foam: basic results. In: Clarke DR, Suresh S, Ward IM, eds. Cellular solids: structure and properties. Cambridge solid-state science series. New York, NY: Cambridge University Press, 2001.

[119] Parfitt AM. Implications of architecture for the pathogenesis and prevention of vertebral fracture. Bone 1992;13:S41-S47.

[120] Hudelmaier M, Kollstedt A, Lochmüller EM, Kuhn V, Eckstein F, Link TM. Gender differences in trabecular bone architecture of the distal radius assessed with magnetic resonance imaging and implications for mechanical competence. Osteoporosis International 2005;16:1124-1133.

[121] Pothuaud L, Benhamou CL, Porion P, Lespessailles E, Harba R, Levitz P. Fractal dimension of trabecular bone projection texture is related to threedimensional microarchitecture. Journal of Bone and Mineral Research 2000;15:691-699.

[122] Mosekilde L. Consequences of the remodelling process for vertebral trabecular bone structure: a scanning electron microscopy study (uncoupling of unloaded structures). Bone and Mineral 1990;10:13-35.

[123] Majumdar S, Kothari M, Augat P, Newitt DC, Link TM, Lin JC, et al. Highresolution magnetic resonance imaging: three-dimensional trabecular bone architecture and biomechanical properties. Bone 1998;22:445-54.

[124] Ulrich D, van Rietbergen B, Laib A, Rüegsegger P. The ability of threedimensional structural indices to reflect mechanical aspects of trabecular bone. Bone 1999;25:55-60. 
[125] Kabel J, Rietbergen Bv, Odgaard A, Huiskes R. Constitutive relationships of fabric, density, and elastic properties in cancellous bone architecture. Bone 1999;25:481-6.

[126] Gomberg BR, Wehrli FW, Vasilić B, Weening RH, Saha PK, Song HK, et al. Reproducibility and error sources of micro-MRI-based trabecular bone structural parameters of the distal radius and tibia. Bone 2004;35:266-276.

[127] Manjón JV, Coupé P, Buades A, Fonov V, Collins DL, Robles M. NonLocal MRI Upsampling. Medical Image Analysis 2010, in press.

[128] Alberich-Bayarri A, Marti-Bonmati L, Pérez MA, Sanz-Requena R, Moratal D. High resolution 3T MRI compared to 64 MDCT and micro CT in the trabecular bone characterization of an adult sheep tibia. A preliminary analysis. 26th Annual Meeting ESMRMB, Antalya, Turkey, 2009.

[129] Kanis JA. Diagnosis of osteoporosis and assessment of fracture risk. Lancet 2002;359:1929-1936.

[130] Schuster DP. The Opportunities and Challenges of Developing Imaging Biomarkers to Study Lung Function and Disease. American Journal of Respiratory and Critical Care Medicine 2007;176:224-230.

[131] Mueller KH, Trias A, Ray RD. Bone density and composition: age-related and pathological changes in water and mineral content. Journal of Bone and Joint Surgery 1966;48:140-148.

[132] Garner E, Lakes R, Lee T, Swan C, Brand R. Viscoelastic dissipation in compact bone: implications for stress-induced fluid flow in bone. Journal of Biomechanical Engineering 2000;122:166-172.

[133] Fernandez-Seara MA, Wehrli SL, Wehrli FW. Diffusion of exchangeable water in cortical bone studied by nuclear magnetic resonance. Biophysical Journal 2002;82:522-529.

[134] Techawiboonwong A, Song HK, Leonard MB, Wehrli FW. Cortical Bone Water: In Vivo Quantification with Ultrashort Echo-Time MR Imaging. Radiology 2008;248:824-833. 


\section{Publications}

\section{Thesis related publications}

\section{International journals}

- Alberich-Bayarri A, Marti-Bonmati L, Sanz-Requena R, Belloch E, Moratal D. In Vivo Trabecular Bone Morphological and Mechanical Relationship using High Resolution 3T Magnetic Resonance Imaging. American Journal of Roentgenology. 2008. 191(3):721-726. (IF: 2,94 [JCR: 2008]).

- Alberich-Bayarri A, Moratal D, Escobar JL, Rodríguez JC, Vallés A, Marti-Bonmati L, Más J, Mano JF, Monleón Pradas M, J.L. Gómez, M. Salmerón. Micro-computed tomography and micro-finite element modeling for evaluating polymer scaffolds architecture and their mechanical properties. Journal of Biomedical Materials and Research: Part B. Applied Biomaterials. 2009. 91:191-202. (IF: 2,19 [JCR: 2009]).

- Alberich-Bayarri A, Marti-Bonmati L, Pérez MA, Sanz-Requena R, Lerma-Garrido JJ, García-Martí G, Moratal D. Assessment of 2D and 3D Fractal Dimension Measurements of Trabecular Bone from High-Spatial Resolution Magnetic Resonance Images at 3 Tesla. Medical Physics. 37:4930-4937. (IF: 2,70 [JCR: 2009]).

\section{National journals}

- Nieto L, Moratal D, Marti-Bonmati L, Alberich-Bayarri A, Galant J. Morphological characterization of trabecular bone structure using high resolution magnetic resonance imaging. Radiología. 2008; 50:401-408.

\section{Book chapters}

- Martí-Bonmatí L, Sanz Requena R, García Martí G, Flórez YN, Alberich Bayarri A. Modelado de sistemas dinámicos en imagen médica. In: Ingeniería Biomédica, Imágenes Médicas. Sistemas Dinámicos. Servicio de Publicaciones de la Universidad de Castilla-La Mancha, 2008. 
- Martí-Bonmatí L, Sanz Requena R, García Martí G, Alberich Bayarri A. Biomarcadores de imagen: su importancia en la imagen médica. In: Ingeniería Biomédica, Nuevas técnicas de procesado de imágenes médicas. Servicio de Publicaciones de la Universidad de Castilla-La Mancha, 2009.

- Alberich-Bayarri A, Salmerón-Sánchez M, Pérez M.A., Moratal D. Microfinite Element Modeling for Evaluating Polymer Scaffolds Architecture and their Mechanical Properties from microComputed Tomography. In: Finite Element Analysis. Sciyo, Rijeka, Croatia, 2010. ISBN: 978-953-307-123-7.

- Alberich-Bayarri A, Martí-Bonmatí L, Pérez M.A., Lerma JJ, Moratal D. Finite Element Modeling for a Morphometric and Mechanical Characterization of Trabecular Bone from High Resolution Magnetic Resonance Imaging. In: Finite Element Analysis. Sciyo, Rijeka, Croatia, 2010. ISBN: 978-953-307-123-7.

\section{International conference proceedings}

- Alberich-Bayarri A, Nieto-Charques L, Moratal-Pérez D, Martí-Bonmatí L, Vallés-Lluch A, Rieta JJ. Clinical Software for the Assessment of Trabecular Bone Disease in Distal Radius Based on a Magnetic Resonance Structural Analysis. 29th Annual International Conference. IEEE Engineering in Medicine and Biology Society. 2073-2076. August 23-26, 2007. Lyon, France.

- Alberich-Bayarri A, Moratal-Pérez D, Martí-Bonmatí L, SalmerónSánchez M, Vallés-Lluch A, Nieto-Charques L, Rieta JJ. Volume Mesh Generation and Finite Element Analysis of Trabecular Bone Magnetic Resonance Images. 29th Annual International Conference. IEEE Engineering in Medicine and Biology Society. August 23-26, 2007. Lyon, France.

- Marti-Bonmati L, Alberich-Bayarri A, Moratal D, Sanz-Requena R. MR elastical characterization of trabecular bone relates to bone volumen ratio and is influenced by gender. European Congress of Radiology (ECR), Annual Scientific Meeting. C-625. March 7-11, 2008. Vienna, Austria.

- Alberich-Bayarri A, Marti-Bonmati L, Sanz-Requena D, Lerma-Garrido JJ, Moratal D. Fractal Dimension Characterization of 3T MR Trabecular 
Bone Images. European Society for Magnetic Resonance in Medicine and Biology (ESMRMB 08). 849. October 2-4, 2008. Valencia, Spain.

- López Orive JJ, Moratal D, Alberich Bayarri A, Martí-Bonmatí L, Salmerón Sánchez M. 3D in-vivo trabecular bone anisotropy and orientation analysis using 3T MR images. European Society for Magnetic Resonance in Medicine and Biology (ESMRMB 08). 719. October 2-4, 2008. Valencia, Spain.

- Alberich-Bayarri A, Marti-Bonmati L, Pérez MA, Sanz-Requena R, Moratal D. High resolution 3T MRI compared to 64 MDCT and micro CT in the trabecular bone characterization of an adult sheep tibia. A preliminary analysis. European Society for Magnetic Resonance in Medicine and Biology (ESMRMB 09). 504. October 1 - 4, 2009. Antalya, Turkey.

- Alberich-Bayarri A, Marti-Bonmati L, Pérez MA, Sanz-Requena R, Garcia-Marti D, Lerma-Garrido JJ, Moratal D. 3D Fractal Dimension of 3T MR-derived trabecular bone structure is highly reproducible and better differentiates healthy from osteoporotic subjects. ECR 2010. March 4-8, 2010. Viena, Austria.

\section{Other publications}

\section{Journals}

- Martí-Bonmatí L, Sanz-Requena R, Alberich-Bayarri A. Pharmacokinetic MR analysis of the cartilage is influenced by field strength. Eur J Radiol. 2008;67:448-452.

- Sanz Requena R, Martí Bonmatí L, Alvarez C, García G, Pellicer A, Alberich-Bayarri A, Moratal D. [Magnetic resonance for evaluating the response to treatment for ovarian hyper stimulation syndrome: comparison of pharmokinetic models.]. Radiologia. 2009;51:176-182.

- Martí-Bonmatí L, Sanz-Requena R, Rodrigo JL, Alberich-Bayarri A, Carot JM. Glucosamine sulfate effect on the degenerated patellar cartilage: preliminary findings by pharmacokinetic magnetic resonance modeling. Eur Radiol. 2009;19:1512-1518. 
- Erturk SM, Alberich-Bayarri A, Herrmann KA, Marti-Bonmati L, Ros PR. Use of 3.0-T MRI for evaluation of the abdomen. Radiographics. 2009;29:1547-1563.

- Sanz-Requena R, Martí-Bonmatí L, Hervás V, Vega M, Alberich-Bayarri A, García-Martí G, Carot JM. Modification of longitudinal relaxation time (T1) as a biomarker of patellar cartilage degeneration. Radiologia. 2010; 52:221-227. 


\section{Abbreviated terms}

\begin{tabular}{|c|l|}
\hline ADC & Analog-to-digital converter \\
\hline ANOVA & Analysis of Variance \\
\hline BMD & Bone Mineral Density \\
\hline BMU & Basic Multicellular Unit \\
\hline BV/TV & Bone Volume to Total Volume ratio \\
\hline CISS & Constructive Interference in Steady-State \\
\hline D & Fractal Dimension \\
\hline D2D & 2D Fractal Dimension \\
\hline D3D & 3D Fractal Dimension \\
\hline DA & Degree of Anisotropy \\
\hline DICOM & Digital Imaging and Communication in Medicine \\
\hline DTA & Digital Topological Analysis \\
\hline DXA & Dual-emission X-Ray Absorptiometry \\
\hline EappX & Compressive Young's modulus in ' $x$ ' direction \\
\hline EappY & Compressive Young's modulus in 'y' direction \\
\hline EappZ & Compressive Young's modulus in 'z' direction \\
\hline FBM & Fractional Brownian Motion \\
\hline FE & Finite Element \\
\hline FID & Free Induction Decay \\
\hline FLASE & Fast Large-Angle Spin-Echo \\
\hline GRE & Gradient Echo \\
\hline GVF & Gradient Vector Flow \\
\hline HR-pQCT & High-Resolution Peripheral Quantitative Computed Tomography \\
\hline
\end{tabular}




\begin{tabular}{|c|l|}
\hline HSV & Hue Saturation Value \\
\hline IGES & Initial Graphics Exchange Specification \\
\hline LTA & Local Thresholding Algorithm \\
\hline MDCT & Multi-Detector Computed Tomography \\
\hline MIL & Mean Intercept Length \\
\hline MRI & Magnetic Resonance Imaging \\
\hline PCA & Principal Component Analysis \\
\hline ppm & Parts per million \\
\hline PSF & Point Spread Function \\
\hline RF & Radiofrequency \\
\hline RMS_CoV & Root Mean Squares Coefficient of Variance \\
\hline ROI & Region of Interest \\
\hline SCR & Surface-to-Curve Ratio \\
\hline SD & Standard Deviation \\
\hline SE & Spin-Echo \\
\hline SEM & Scanning Electron Micrograph \\
\hline SENSE & Sensitivity Encoding \\
\hline SERM & Selective Estrogen Receptor Modulators \\
\hline SNR & Signal-to-Noise Ratio \\
\hline SSFP & Steady-State Free Precession \\
\hline STL & Standard Tesselation Language \\
\hline Tb.N & Trabecular Number \\
\hline Tb.Sp & Trabecular Separation \\
\hline Tb.Th & Trabecular Thickness \\
\hline TE & Echo Time \\
\hline
\end{tabular}


Abbreviated terms

\begin{tabular}{|c|l|}
\hline TEI & Total Erosion Index \\
\hline TR & Repetition Time \\
\hline UCT & Micro-Computed Tomography \\
\hline VOI & Volume of Interest \\
\hline WHO & World Health Organization \\
\hline
\end{tabular}




\section{Appendix}

\section{Developed software}

All the algorithms presented in this thesis have been developed in Matlab R2007a (The Mathworks Inc., Natick, MA, USA). A software application was implemented in order to integrate all the methodologies in a common platform. A database to store the quantified results for each case and the routine to build a final report were also included in the software. The current workflow for the trabecular bone characterization of a given patient can be appreciated in figure A.1.

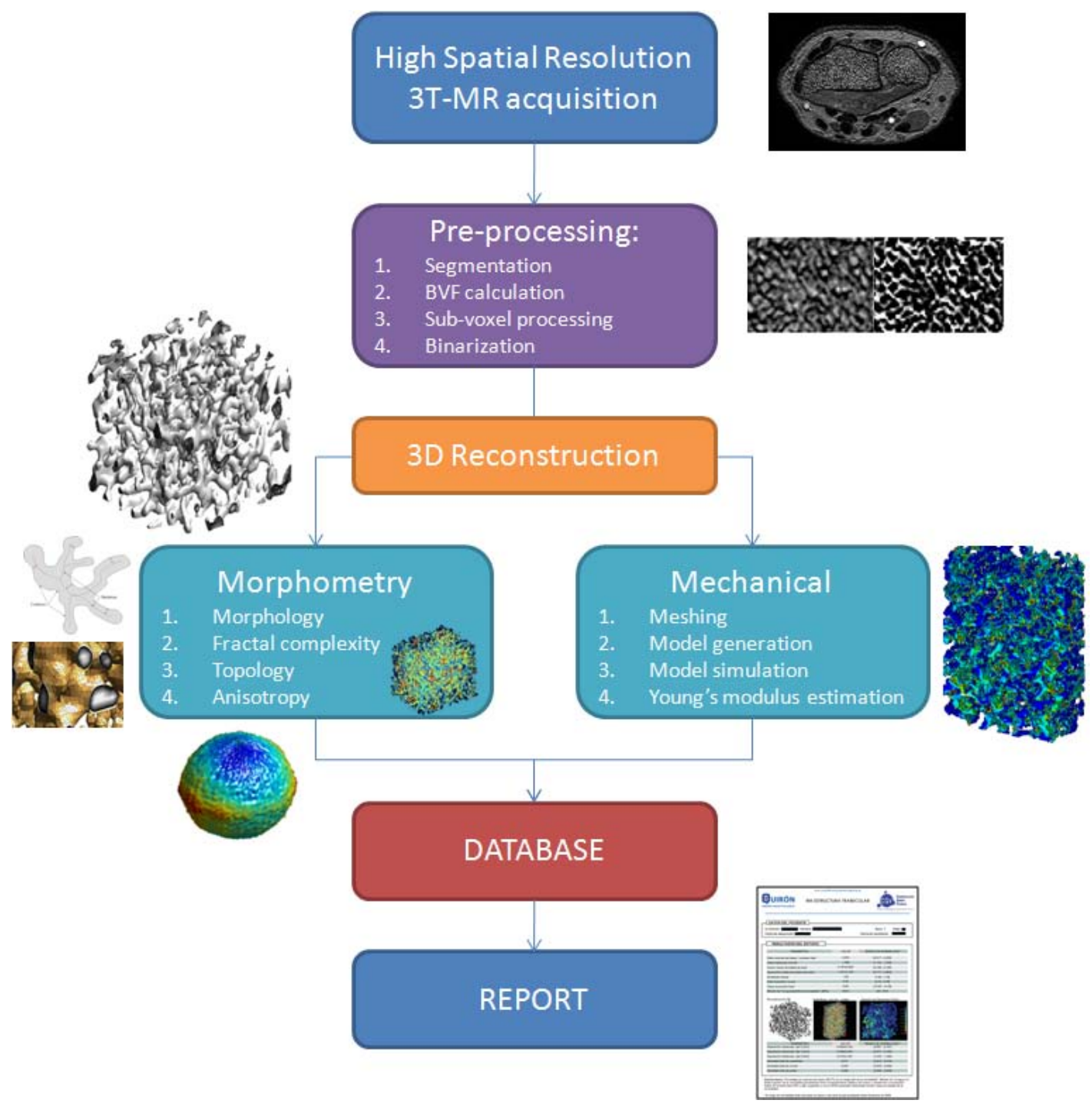

Figure A.1. Current clinical workflow for the trabecular bone morphometry and mechanical characterization. 
A screenshot of the developed software application may be observed in figure A.2. All the methods have been integrated and have the capability of being launched independently in order to evaluate the effect of the different algorithms. An 'AUTO' button may be appreciated on the left side of the image, that serves to launch an automated analysis for both morphometry and mechanical characterization of bone.

A specific module to read data from CT and $\mu \mathrm{CT}$ acquisitions has been also included in the software.

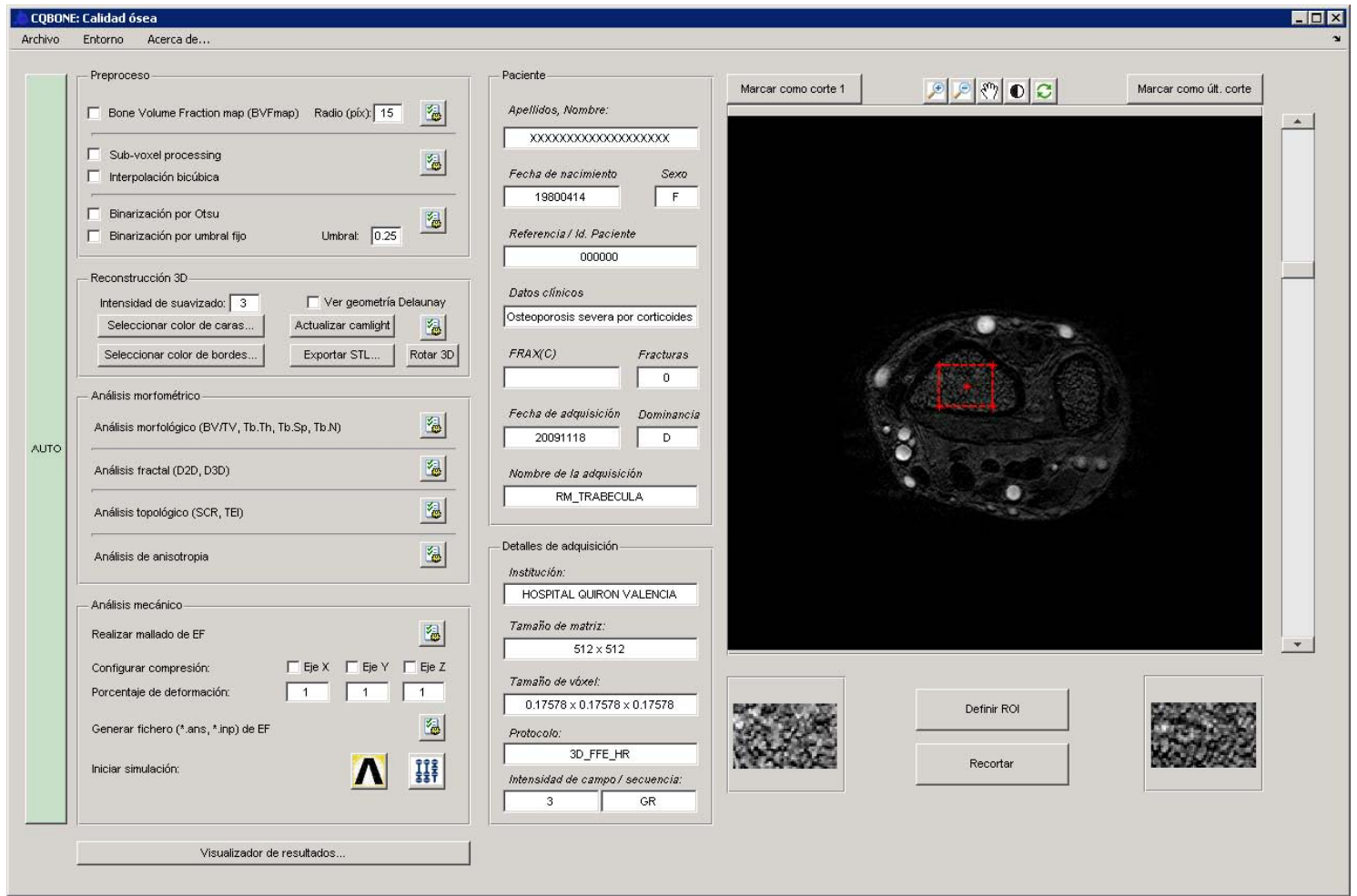

Figure A.2. Screenshot of the integrated software application for a morphometry and mechanical characterization of trabecular bone. 